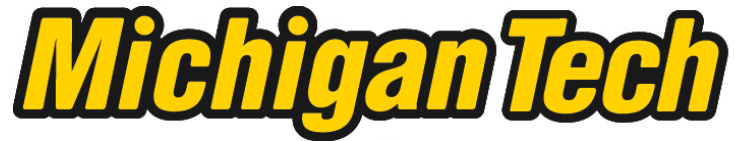 \\ Michigan Technological University Create the Future Digital Commons @ Michigan Tech
}

Dissertations, Master's Theses and Master's Reports - Open

Dissertations, Master's Theses and Master's

Reports

2015

\section{MULTI-MODE AND SINGLE MODE POLYMER WAVEGUIDES AND STRUCTURES FOR SHORT-HAUL OPTICAL INTERCONNECTS}

Kevin L. Kruse

Michigan Technological University

Follow this and additional works at: https://digitalcommons.mtu.edu/etds

Part of the Communication Commons, Optics Commons, and the Polymer Chemistry Commons Copyright 2015 Kevin L. Kruse

\section{Recommended Citation}

Kruse, Kevin L., "MULTI-MODE AND SINGLE MODE POLYMER WAVEGUIDES AND STRUCTURES FOR SHORT-HAUL OPTICAL INTERCONNECTS", Dissertation, Michigan Technological University, 2015.

https://doi.org/10.37099/mtu.dc.etds/977

Follow this and additional works at: https://digitalcommons.mtu.edu/etds

Part of the Communication Commons, Optics Commons, and the Polymer Chemistry Commons 


\title{
MULTI-MODE AND SINGLE MODE POLYMER WAVEGUIDES AND STRUCTURES FOR SHORT-HAUL OPTICAL INTERCONNECTS
}

\author{
By \\ Kevin L. Kruse \\ A DISSERTATION \\ Submitted in partial fulfillment of the requirements for the degree of \\ DOCTOR OF PHILOSOPHY \\ In Electrical Engineering
}

MICHIGAN TECHNOLOGICAL UNIVERSITY

2015

(C) 2015 Kevin L. Kruse 
This dissertation has been approved in partial fulfillment of the requirements for the Degree of DOCTOR OF PHILOSOPHY in Electrical Engineering.

Department of Electrical and Computer Engineering

Dissertation Advisor: Dr. Christopher Middlebrook

Committee Member: Dr. Michael Roggemann

Committee Member: $\quad$ Dr. Durdu Guney

Committee Member: $\quad$ Dr. Craig Friedrich

Department Chair: $\quad$ Dr. Daniel R. Fuhrmann 


\section{To Lee \& Diane Kruse}




\section{Table of Contents}

List of Figures.................................................................................................................................... ix

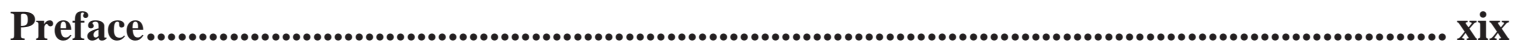

Acknowledgements ............................................................................................................................ xxi

Abstract................................................................................................................................................... xxii

Chapter 1: Optical Interconnects ....................................................................................................1

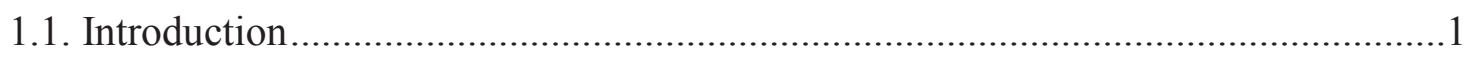

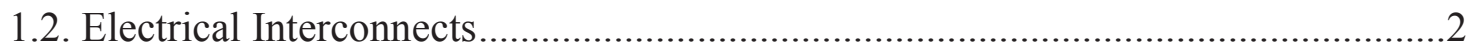

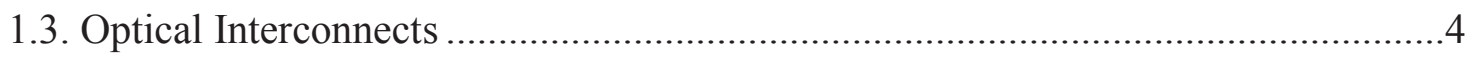

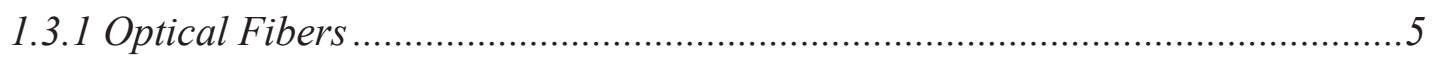

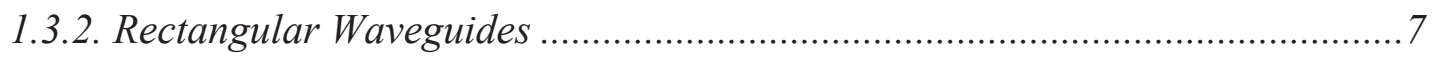

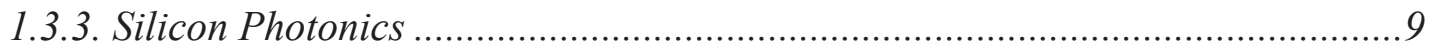

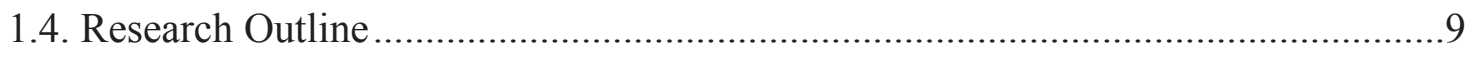

Chapter 2: Polymer Waveguide Theory ………………................................................................15

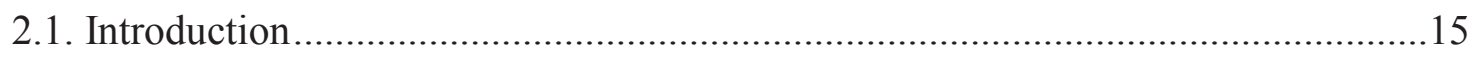

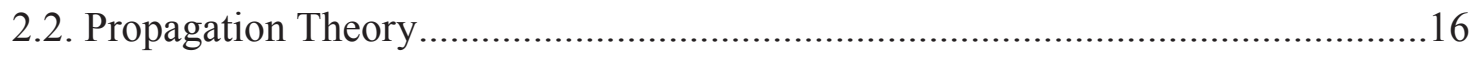

2.2.1. Ray Tracing Method .............................................................................. 16 


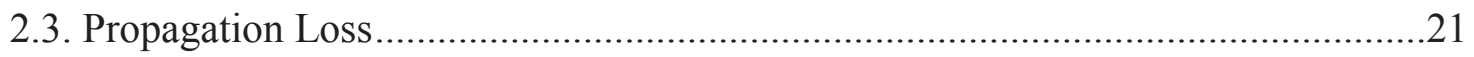

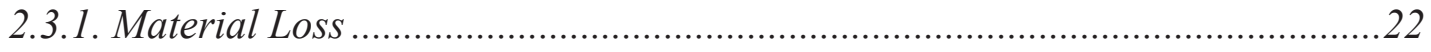

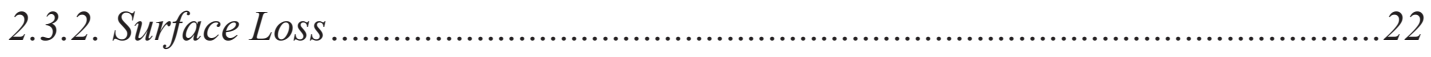

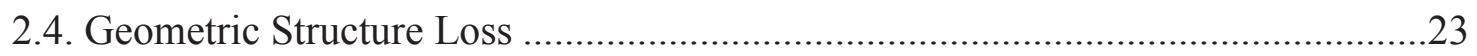

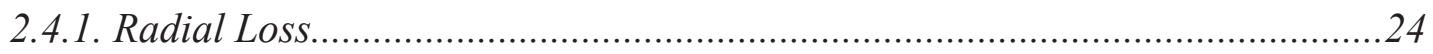

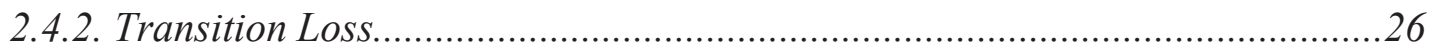

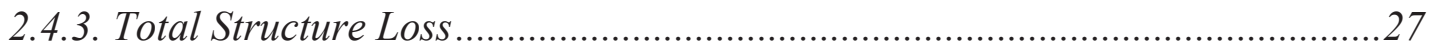

2.5. Dielectric WG Model and Simulation Methods ..............................................28

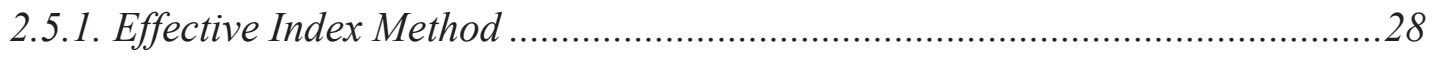

2.5.2. Finite Difference Time Domain ............................................................ 30

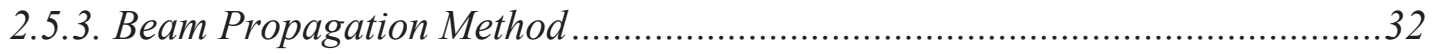

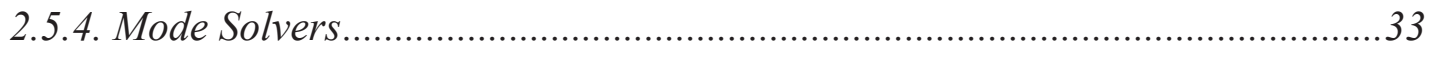

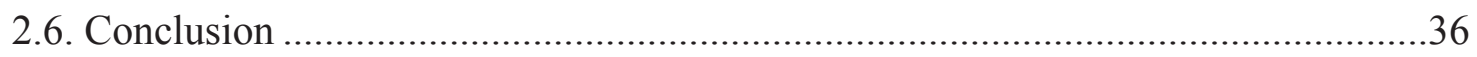

Chapter 3: Waveguide Fabrication and Evaluation..........................................................37

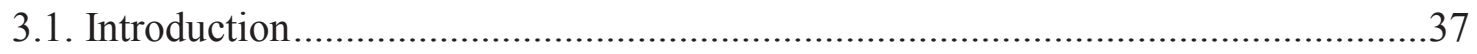

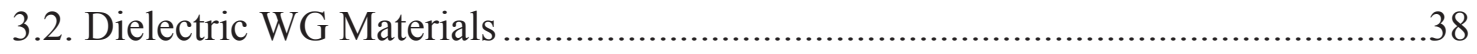

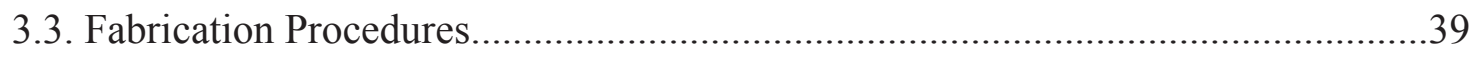

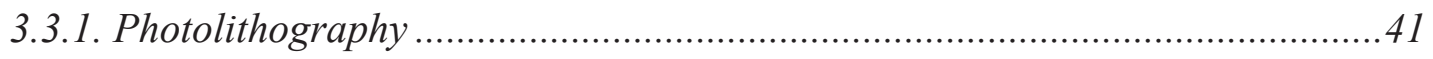


3.4. Testing and Evaluation

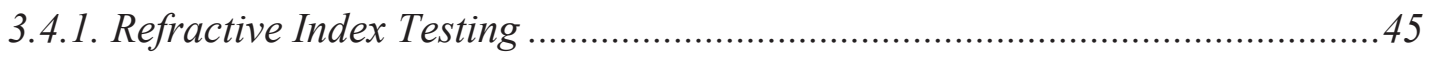

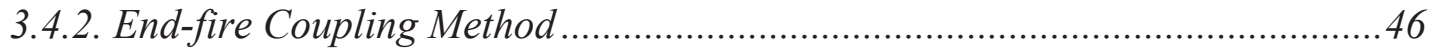

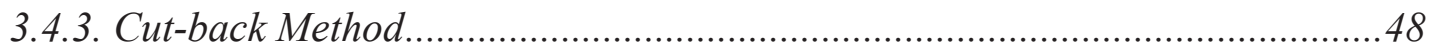

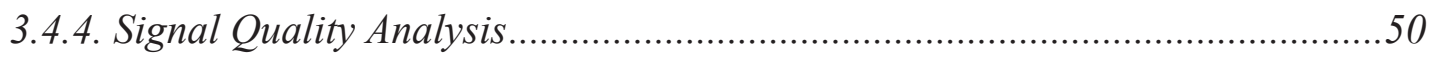

3.5. Fabrication Summary ................................................................................... 51

Chapter 4: Laser Direct Write Method ................................................................................53

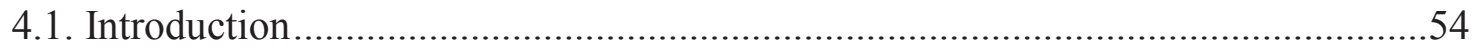

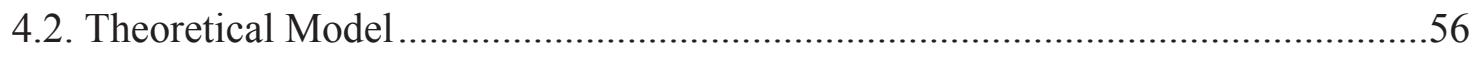

4.3. Experimental Setup Procedure......................................................................58

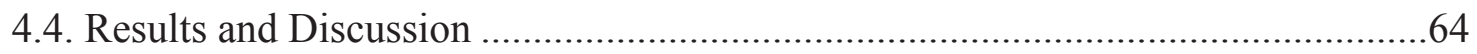

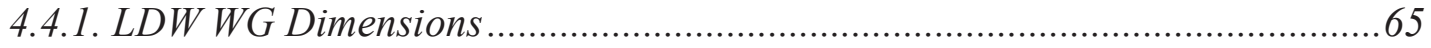

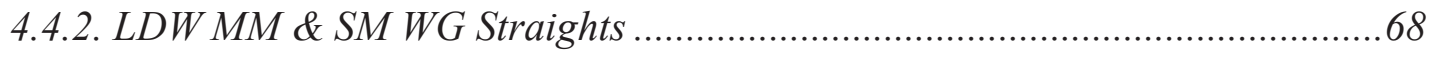

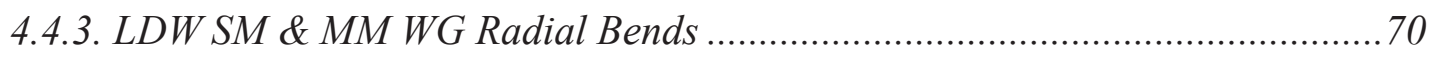

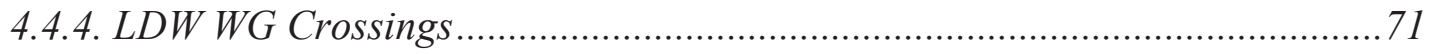

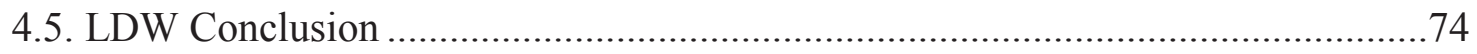

Chapter 5: SM WG S-Bends and Splitters .............................................................................76

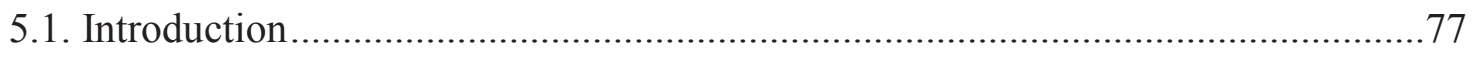

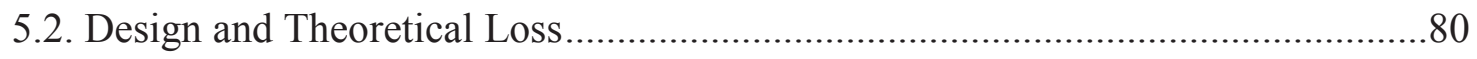


5.3. S-Bend Fabrication

5.4. Photolithographic Results .87

5.4.1. Photolithographic SM WG S-Bends. .87

5.4.2. S-Bend Splitters.

5.4.3. Laser Direct Written SM WG S-Bends. 93

5.4.4. S-Bend Foot-print Requirements 97

5.5. SM WG S-Bend Conclusion. 100

Chapter 6: Polymer WG Tapers .02

6.1. Introduction.

6.2. Polymer WG Taper Theory and Designs..... 106

6.2.1. Taper Theory. 106

6.2.2. Taper Design. 109

6.3. WG Taper Simulation Results 111

6.3.1 Taper Length Requirements......

6.3.2 Coupling Loss 115

6.3.3 Misalignment Tolerances...... 117

6.3.4 Metallic Loss 122

6.4. Polymer WG Taper Experimental Results.

6.4.1 Taper Fabrication. 124 vii 


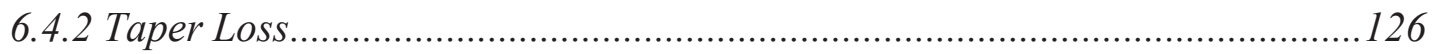

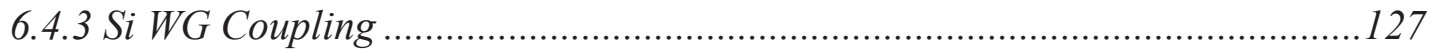

6.4.4 Polymer Taper Loss Optimization ...................................................... 128

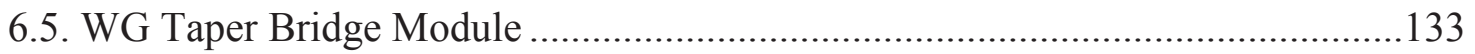

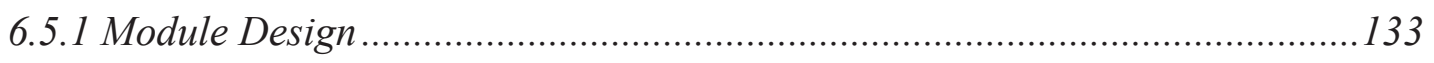

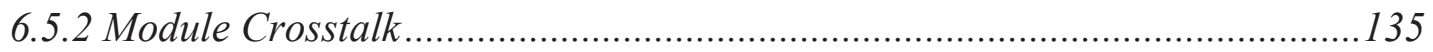

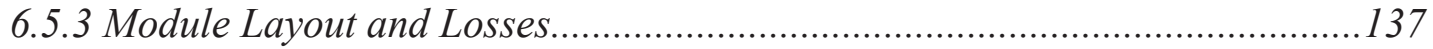

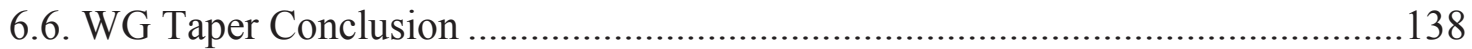

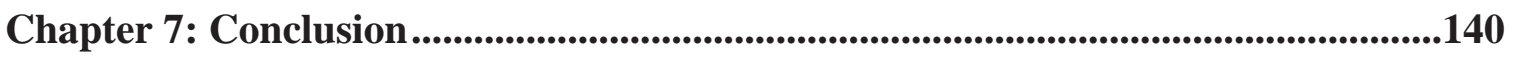

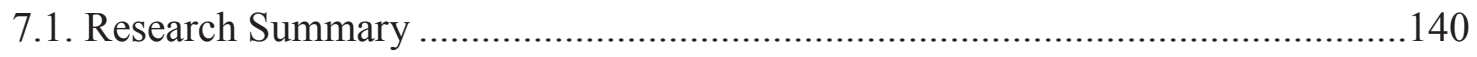

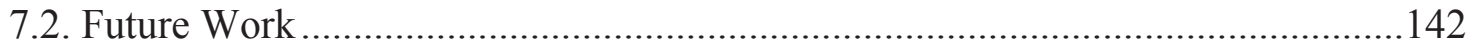

References .........................................................................................................................................144 


\section{List of Figures}

Figure 1.1: Schematic of two parallel electrical transmission lines. 3

Figure 1.2: Optical Fibers with core diameters of $8 \mu \mathrm{m}$ (yellow), $50 \mu \mathrm{m}$ (aqua), and $62.5 \mu m$ (orange). Connector types shown are the SC (yellow), FC (aqual), and LC

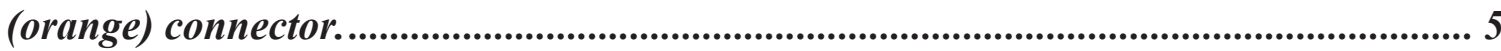

Figure 1.3: Optical interconnect bus of six polymer waveguides in the form of a 1 meter spiral are fabricated on PCB dielectric substrate.............................................8

Figure 1.4 Polymer taper bridge module illustration. ................................................... 10

Figure 2.1: Correlation of incident and refracted angles with different boundary conditions.

Figure 2.2: An illustration showing a ray tracing of a guided ray at the critical angle

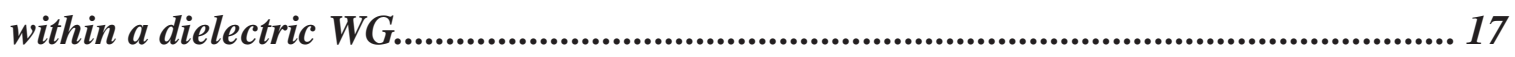

Figure 2.3: The first 4 propagation modes in a multi-mode dielectric slab WG.......... 20

Figure 2.4: Signal Dispersion in a SM (top) and MM (bottom) Fiber......................... 21

Figure 2.5: Transitional and Radial Losses of a propagating mode in a WG Bend.... 24

Figure 2.6: The TEM mode of a $6 \mu \mathrm{m} S M W G$ extends into the cladding in all directions. 29 
Figure 2.7: Assumes negligible modal presence in the shaded corners of the WG crosssection, the Effective Index Method [51] can be utilized to convert a 3D WG into a $2 D$ slab WG for modal approximations.

Figure 2.8: The FDTD method in simulating a Mach-Zehnder interferometer. The peaks and troughs of the electric field are visually observed during a discrete instance in time.

Figure 2.9: The BPM utilized to simulate a MM interference splitter. The propagation power is assumed steady-state.

Figure 2.10: Modes for a partially-etched rib WG solved for using a finite difference mode solver, showing only modes with $H_{y}$ polarization being confined......

Figure 3.1: IR absorption spectrum of polymer WG core material.

Figure 3.2: Spin Curve of UV-curable optical elastomer utilized in polymer WG fabrication. 40

Figure 3.3: WG fabrication process. 41

Figure 3.4: Illustration of the photolithographic process. 42

Figure 3.5: Calculated diffraction patterns at various propagation distances from mask slit of various widths. 44 
Figure 3.6: Prism coupling method conducted on core material spin-coated on a glass

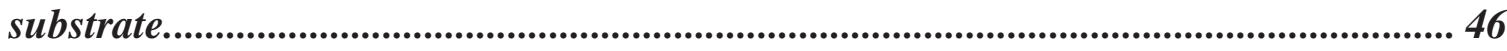

Figure 3.7: The End-fire Coupling Method. ............................................................ 47

Figure 3.8 Cross-section image of a $6 \times 6$ m $S M$ polymer $W G$............................... 48

Figure 3.9 Loss plot for photolithographic SM polymer WGs from the cutback method. 49

Figure 3.10 Normalized measured misalignment loss between a SMF and SM polymer

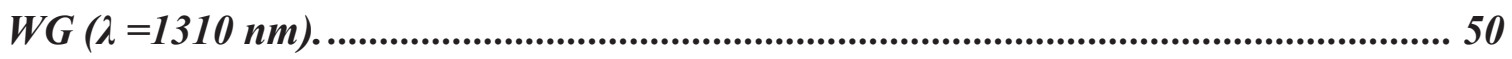

Figure 3.11 Eye diagrams at 10 GBits/sec for SM WG straights (a) and SM WG bends

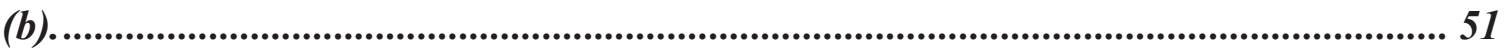

Figure 4.1: Theoretical setup of the $L D W$ process....................................................... 54

Figure 4.2: Gaussian curing model at various power levels in relation to the energy threshold required for polymerization............................................................................ 57

Figure 4.3 UV Absorption Spectrum of the Dow Corning WG Material...................... 58

Figure 4.4 3D Beam profile of focused UV SMF laser output................................... 60

Figure 4.5 2D matched Gaussian fit of focused UV SMF laser output. ...................... 61 
Figure 4.6 Beam divergence observed with increasing $1 / e^{2}$ beam diameter width as the writing height is increased away from focus $(x=0 \mu m)$. 61

Figure 4.7 The laser direct writing setup consists of an air-bearing XYZ movement stage (a) equipped with a lens-focused UV SMF output (b).

Figure 4.8 Cross-sections of symmetric $50 \mu m$ MM (a) and $6 \mu m$ SM (b) WGs fabricated through $\mathrm{LDW}$.

Figure 4.9: WG straights, bends, and crossings are utilized in the design of polymer waveguide shuffleboards.

Figure 4.10 LDW WG widths correlated to $U$ V beam diameters of $60 \mu m$. The polymer core layer thickness applied before LDW was $50 \mu \mathrm{m}$. 66

Figure 4.11 LDW WG widths correlated to $U$ V beam diameters of $20 \mu m$. The polymer core layer thickness applied before $L D W$ and $7 \mu m$. 66

Figure 4.12 LDWWG abnormalities observed: high dwell time (a) and underexposure (b).

Figure 4.13 Radial bend loss for MM WGs $(\lambda=830 \mathrm{~nm})$ (a) and SM WGs $(\lambda=1310$ nm) (b).

Figure 4.14 Top-down image of MMWG crossings fabricated from $L D W$ (a) and photolithography (b). 
Figure 4.15 Top-down image of SM WG crossings fabricated from $L D W(a)$ and

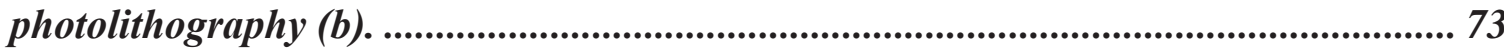

Figure 4.16 LDW WG crossing loss for MM WGs (

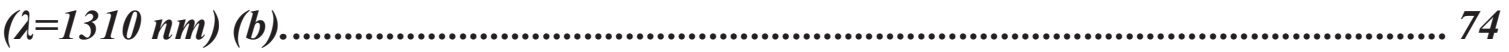

Figure 5.1 Images of S-Bend Utilized in WG fan-out (a) and Mach-Zehnder

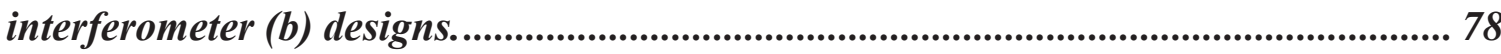

Figure 5.2 S-Bend profile functions.................................................................. 81

Figure 5.3 S-Bend curvature functions. ....................................................................... 82

Figure 5.4 Simulated SM WG $(N A=0.15)$ structure loss for $S$-Bend with a $0.5 \mathrm{~mm}(\mathrm{a})$

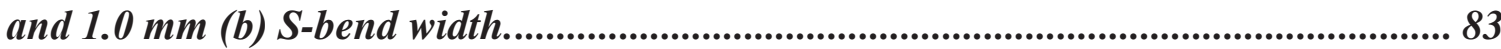

Figure 5.5 Simulated SM WG $(N A=0.35)$ structure loss for $S$-Bend with a $0.5 \mathrm{~mm}(\mathrm{a})$

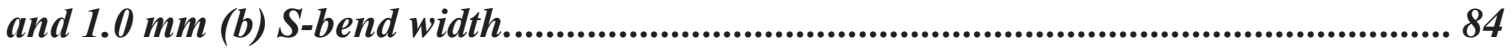

Figure 5.6 Illustration of segmentation utilized in realizing a sinusoidal bend. Shorter segmentation lengths approach the ideal line of the smooth bend. 85

Figure 5.7 Top-down view of 6 mm SM WG straights (a), raised-sine S-bends (b), and

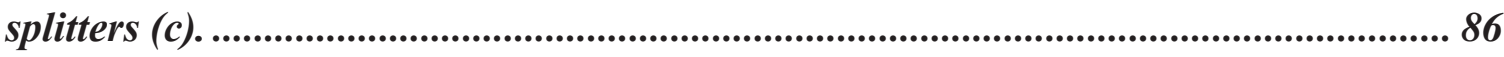

Figure 5.8 Raised-sine S-Bend structure loss shown using theoretical curves (lines)

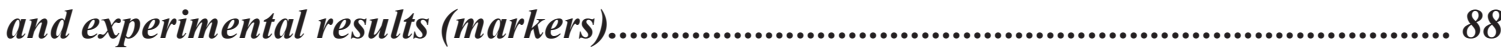


Figure 5.9 S-Bend structure loss for different S-bend designs shown using theoretical curves (lines) and experimental results (markers)..................................................... 88

Figure 5.10 Top-down view of WG S-bend splitters integrated with no splitting taper (a), a $100 \mu \mathrm{m}$ long taper (b), and a $500 \mu \mathrm{m}$ long taper (c). 90

Figure 5.11 Experimental splitting loss as a function of S-bend type and taper length.

Figure 5.12 Theoretical splitting loss as a function of S-bend type and taper length. 92

Figure 5.13 Residual polymer material present within an S-bend splitter.

Figure 5.14 Cosine SM WG S-bends with segmentation lengths of $1000 \mu \mathrm{m}$ (a), 100 $\mu m(b), 10 \mu m(c)$, and $1 \mu m(d)$. 93

Figure 5.15 Experimental loss of SMWG cosine S-bends written at $25 \mathrm{~mm} / \mathrm{s}$ utilizing various segmentation lengths $(\lambda=1310 \mathrm{~nm})$.

Figure 5.16 Experimental loss of SM WG radial arc consecutive and segmented $S$ bends $(\lambda=1310 \mathrm{~nm})$.

Figure 5.17 Structure loss of polymer SM WG S-bends manufactured through laser direct writing $(\lambda=1310 \mathrm{~nm})$.

Figure 5.18 Theoretical (line) and experimental (marker) structure loss of polymer WG radial bends fabricated through photolithography and laser direct writing. 98 
Figure 5.19 Structure, propagation, and total loss of raised-sine S-bends [W=1000 $\mu m]$.

Figure 6.1 Simulated mode profile [ $\lambda=1310 \mathrm{~nm}]$ of $8 \mu \mathrm{m}$ diameter $S M F(a)$ and 400 $\mu m \times 200 \mu m$ Si WG (b). 104

Figure 6.2 Polymer taper bridge module illustration. 106

Figure 6.3 Simulated mode width in polymer WGs as a function of $N A$ and tapered WG width $(\lambda=1310 \mathrm{~nm})$. 107

Figure 6.4 Si WG modal excitation in polymer WG tapers with a $0.5 \mu m$ (a) and 3.0 $\mu m$ (b) WG taper width. 108

Figure 6.5 Vertical taper illustration 110

Figure 6.6 Multi-layer taper illustration. 110

Figure 6.7 Taper loss as a function of taper length for vertical tapers (0.5 $\mu \mathrm{m}$ Taper Height, $\lambda=1310 \mathrm{~nm})$. 113

Figure 6.8 Taper loss as a function of taper length for multi-layer tapers $(0.5 \mu \mathrm{m}$ Taper Height, $\lambda=1310 \mathrm{~nm})$. 113

Figure 6.9 Directional taper loss in multi-layer tapers as a function of taper length. (0.5 $\mu \mathrm{m}$ Taper Width, $0.5 \mu \mathrm{m}$ Taper Height, $\lambda=1310 \mathrm{~nm})$. 
Figure 6.10 Multi-layer taper loss as a function of lithographic resolution $(\lambda=1310$

nm).

Figure 6.11 Mode profiles for SM Si Wire (a) and Si Rib (b) WGs $(\lambda=1310 \mathrm{~nm})$....... 116

Figure 6.12 Coupling loss between Si wire WGs and SMFs utilizing vertical tapered WGs with various tip dimensions $(\lambda=1310 \mathrm{~nm})$. 118

Figure 6.13 Coupling loss between Si wire WGs and SMFs utilizing multi-layer tapered WGs with various tip dimensions $(\lambda=1310 \mathrm{~nm})$. 118

Figure 6.14 Coupling loss between Si rib WGs and SMFs utilizing vertical tapered WGs with various tip dimensions $(\lambda=1310 \mathrm{~nm})$. 119

Figure 6.15 Coupling loss between Si rib WGs and SMFs utilizing multi-layer tapered WGs with various tip dimensions $(\lambda=1310 \mathrm{~nm})$. 119

Figure 6.16 Lateral taper misalignment loss coupling with Si wire waveguides $(\lambda=1310$ nm). 120

Figure 6.17 Lateral taper misalignment loss coupling with Si rib waveguides $(\lambda=1310$ nm). 121

Figure 6.18 Gap loss when coupling with Si wire waveguides $(\lambda=1310 \mathrm{~nm})$.

Figure 6.19 Gap loss when coupling with Si rib waveguides $(\lambda=1310 \mathrm{~nm})$. 122 
Figure 6.20 Mode profile of the $\mathrm{TE}_{0}$ and $\mathrm{TM}_{0}$ of an asymmetrical gold/polymer/air Waveguide.

Figure 6.21 Spin Curve of diluted (75\% solvent) UV-curable optical elastomer utilized in polymer WG tapers. 125

Figure 6.22 Cross sections of fabricated multi-layer taper along the length of the polymer WG device. 126

Figure 6.23 Measurement setup used to determine taper loss due to sidewall roughness and gold absorption. 127

Figure 6.24 Measurement setup used to determine polymer taper coupling loss with Si WGs 128

Figure 6.25 FE-SEM images of multi-layer polymer tapers with 3 layers (a), 2 layers (b), and 1 layer (c). 130

Figure 6.26 Simulated propagation loss from sidewall scattering for air-clad WGs as a function of $W G$ width and sidewall roughness $\left(\lambda=1310 \mathrm{~nm}, L_{c}=3 \mu \mathrm{m}\right)$. 131

Figure 6.27 Overlapping tapers exhibiting under-exposure effects from diffractionlimited fabrication.

Figure 6.28 Polymer taper bridge module incorporated with WG tapers (a), isolation WGs (b) WG S-Bends (c), and end WGs (d).The shaded region in section (a) represents a low-index medium for high $N A$ WG tapers. 134 
Figure 6.29 Waveguide module cross-talk levels over multiple individually excited WGs. Box plots show the Q1, mean, and Q3 levels of cross-talk in simulation. Whiskers show full range of the data. ............................................................................. 136 


\section{Preface}

This dissertation reflects information published by the author during his the Ph.D. degree research in the Electrical and Computer Engineering Department at Michigan

Technological University. The research contributes to the design, simulation, fabrication, and evaluation of single mode polymer waveguides for high-speed optical communication applications. The work presented in this dissertation includes work from previously published articles in Chapters 3, Chapter 4, and Chapter 5.

Research in Chapters 3 and 5 were previously published in Taylor \& Francis journal Journal of Modern Optics as "Fan-out routing and optical splitting techniques for compact optical interconnects using single-mode polymer waveguides". As the first author, I have identified and optimized efforts in the design and fabrication of waveguide S-bends and splitters required for many applications in optical waveguiding devices.

Chapters 4 and 5 reflect work from a previously published Elsevier journal Photonic Nanostructures: Fundamentals and Applications titled "Laser-direct writing of single mode and multi-mode polymer step index waveguide structures for optical backplanes and interconnection assemblies." The author has developed a model in the fabrication of laser direct written polymer waveguides and experimentally verified comparable functionality to photolithographic builds. 
The author's advisor, Dr. Christopher Middlebrook, has been a significant contributor in outlining project goals, reviewing research progress, and peer-review editing prior to journal submission and revisions for all the listed journal articles above. 


\section{Acknowledgements}

First and foremost, this work would not have been possible without the efforts of my advisor, Dr. Middlebrook. Thank you for your undying support towards our contributions to the academic community.

Dow Corning has also played a pivotal role throughout my graduate studies including monetary contribution and material donations. Thank you to Brandon, David, and William for enabling industrial collaboration opportunities in sync with my research.

The Micro-Fabrication Facility (MFF) at Michigan Tech has been very supportive in my experimental work. A special thanks to William, Thomas, Mark, and Mike for their tutorage and troubleshooting of various fabrication equipment on numerous occasions.

Last but not least, my family has been nothing but supportive ever since I left the farm in pursuit of a career in engineering. The unconditional love of Lee, Diane, Katrina, and Kayle has been a constant source of relief and joy in my life through the numerous academic, financial, personal, and marital strains that I have experienced during my Ph.D. studies. 


\section{Abstract}

Single mode and multi-mode polymer optical waveguides are a viable solution for replacing copper interconnects as high speed and large bandwidth short-haul optical interconnects in next-generation supercomputers and data servers. A precision laser direct writing method is implemented for producing various single mode and multi-mode polymer waveguide structures and their performance is evaluated experimentally showing agreement with theoretically developed models. The laser direct writing method is the optimal solution for low-rate cost-effective prototyping and large area panel production.

A single mode polymer waveguide bridge module for silicon to glass optical fibers is designed, modeled, fabricated, and measured. The bridge module is designed for waveguide pitch control and low coupling loss from high-density silicon photonic interconnects within CMOS devices and optical silica fibers for long-haul low-loss transmission. A fan-out structure using waveguide S-bend structures is utilized to perform pitch control. Optical coupling within the bridge module is achieved through a novel polymer taper structure to reduce the numerical aperture mismatch between silicon waveguides and silica fibers. Research and development has been implemented into the theoretical understanding and experimental assessments of solving practical interconnect challenges for commercial realization of polymer waveguides. 


\section{Chapter 1: Optical Interconnects}

\subsection{Introduction}

Since the invention of the telegraph data transmission has vastly improved due to political and consumer demand for advances in communication technology. Electronic devices and computers continued to drive the development of digital communication where isolated processors required a means to transmit digital signals between devices and external peripherals through printed circuit boards and copper-based wiring. Copperbased transmission lines exhibit decreasing theoretical bandwidth limits present as PCB routing becomes increasingly denser. Electronic data transmission rates are currently limited within large-scale computing devices where copper-based printed circuit boards (PCBs) are unable to transmit high bandwidth electrical signals without significant levels of loss and distortion.

Optical interconnects ( $>10 \mathrm{Gbits} \cdot \mathrm{m} / \mathrm{sec}$ ) overcome bandwidth limitations and become a cost-efficient method of communication channels in terms of lower material costs from smaller devices and cheaper operating costs in low-loss optical signal transmission. Fiber-optic cables have replaced copper-wire based technologies in long-haul (>1 m) communication for their advantages in high bandwidth, low loss $(0.2 \mathrm{~dB} / \mathrm{km})$, immunity to EM interference, and data security. Rectangular waveguides (WGs) are an alternate form of optical interconnects that are more applicable for short-haul interconnects. In this Chapter, electrical transmission lines and their physical limitations are presented and 
evaluated. Optical interconnects and their advantages are then discussed for both optical fibers and rectangular WGs. Finally, optical interconnect technologies are then evaluated for their applicability in printed circuits.

\subsection{Electrical Interconnects}

From supercomputers to cellphones copper interconnects within PCBs are utilized to interconnect integrated circuits and peripherals required for system operation. With the economic desire to increase device speed, efficiency, and portability the bandwidth density required within PCBs has increased. Current demands require PCB copper interconnects to be placed in closer proximity laterally and layered using thinner dielectric substrates. These alterations both increase low-pass filtering that occurs within PCB copper interconnects.

An electrical transmission line can be represented by a simple circuit consisting of conductive lines, as displayed in Figure 1.1. Two coppers lines separated by a PCB dielectric exhibit a level of capacitance inversely proportional to the proximity distance resulting in a low-pass filter. The lines also exhibit inductance due the interaction of the induced magnetic flux with adjacent conducting lines.

As the data transmission rate is increased a higher percentage of the electrical power within the high-frequency signal will be absorbed by the transmission line. The absorbed power is re-emitted as heat within the PCB. Input power requirements to effectively send a clear signal through the transmission line exponentially increases with the frequency of 
data transmission. Higher power levels also drastically increase working temperatures, eventually resulting in thermal degradation and device failure.

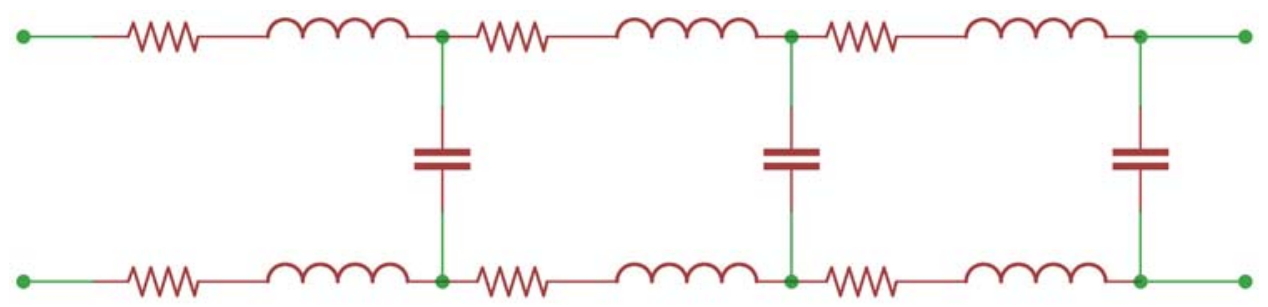

Figure 1.1: Schematic of two parallel electrical transmission lines.

Copper-based interconnects also degrade the transmitted signal quality. Time-varying electrical signals and their induced magnetic fields couple into nearby electrical transmission lines, resulting in both signal loss in the main line and unwanted induced current noise in the form of cross-talk in neighboring transmission lines. This mutual coupling reduces the signal-to-noise ratio (SNR) in both transmission lines. The SNR within copper-based interconnects decreases as transmission frequency and interconnect density increase.

Physical limitations have to be taken into account when designing high-end PCBs to ensure error-free data transmission between electronic devices. Manufacturers have utilized advanced wiring algorithms and advanced dielectrics to optimize copper interconnect placement to optimize transmission frequency. Phenomenon associated with high speed copper-based interconnects are known to limit the bandwidth of modern day PCB designs to $<1$ Gbits $\cdot \mathrm{m} / \mathrm{sec}[1]$. Unfortunately, advancements in interconnect 
technology are fast approaching physical limits in copper-based interconnects and alternative methods of transmission are required.

\subsection{Optical Interconnects}

Optical interconnects are a key technology in realizing high-bandwidth operation in future electronic communication [2-4]. Optical interconnects utilize a transparent dielectric medium (semiconductor/glass/polymer) to confine and transmit optical pulses of light. The transmission loss of optical interconnects is independent of data transmission frequency since optical interconnects do not exhibit the capacitance and inductance effects present in electronic interconnects. Furthermore, optical interconnects can transmit multiple signals at specified wavelengths, $\lambda$, in a single transmission line through the use of wavelength-division-multiplexing (WDM). The use of optical interconnects result in bandwidths ( $\geq 100 \mathrm{Gbits} \cdot \mathrm{m} / \mathrm{sec}$ ) that are orders of magnitude higher than electrical interconnects $(1 \mathrm{Gbits} \cdot \mathrm{m} / \mathrm{sec})$.

Optical interconnects can be manufactured in a number of methods, but they can be classified into two categories: optical fibers and rectangular WGs. While both designs operate under the same physical phenomenon of total internal reflection their economic feasibility separates them for uses in different applications. Optical fibers are glass fibers that are commonly used for long-haul data transmission applications due to their low (0.2 $\mathrm{dB} / \mathrm{km}$ ) low characteristics. Rectangular WGs are geared toward short-haul digital communication since their losses are higher than their counterparts but exhibit cost advantages and high manufacturing through-put for PCB compatibility. 


\subsubsection{Optical Fibers}

In the 1970s long-haul electrical transmission lines were being replaced with silica glass optical fibers. Exhibiting low propagation loss of $0.2 \mathrm{~dB} / \mathrm{km}$ at $\lambda=1550 \mathrm{~nm}$ optical fibers require significantly lower requirements in terms of power consumption and signal conditioning. Furthermore, silica glass optical fibers are environmentally resistant and immune to corrosion, improving device longevity. The characteristics of the silica glass optical fiber are controlled with alterations in fiber dimensions, dopant types, and doping profiles. Optical fibers come in a variety of designs, usually labeled by color, to optimize propagation losses, bandwidths limits, and coupling loss for the desired application. A visual of optical fibers with commercially-available connectors are shown in Figure 1.2.

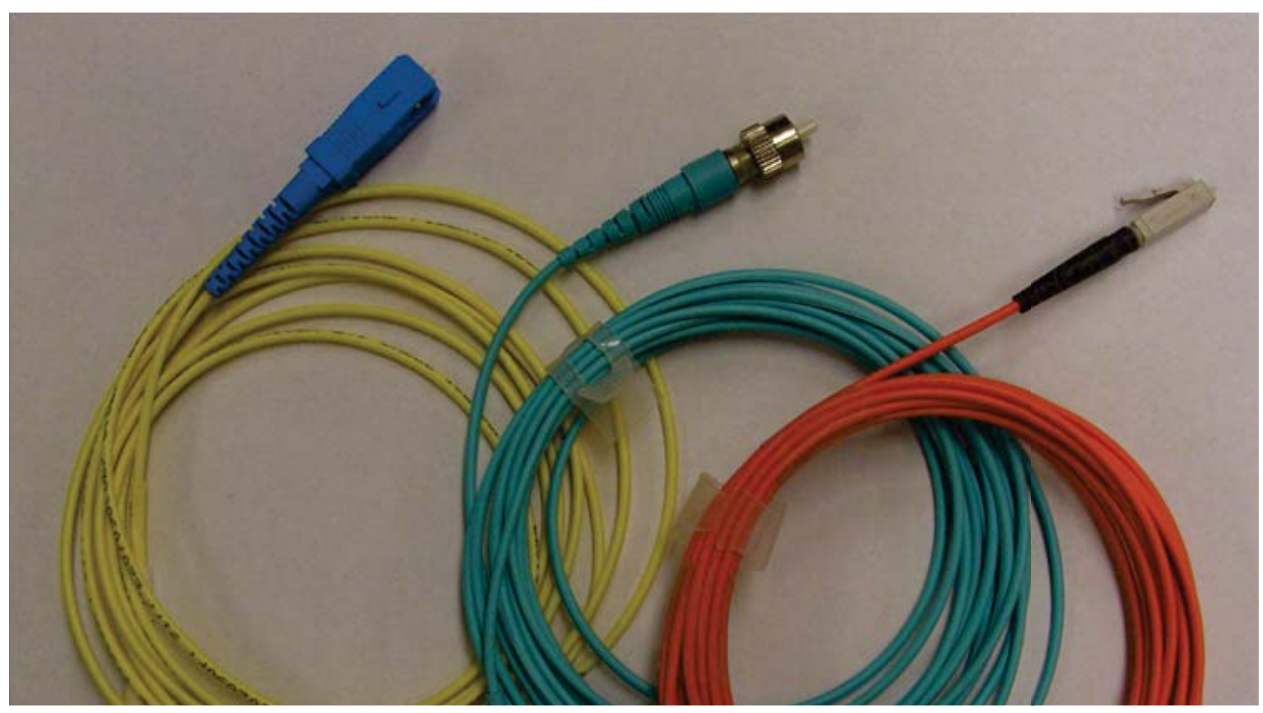

Figure 1.2: Optical Fibers with core diameters of $8 \mu \mathrm{m}$ (yellow), $50 \mu \mathrm{m}$ (aqua), and $62.5 \mu \mathrm{m}$ (orange). Connector types shown are the SC (yellow), FC (aqual), and LC (orange) connector. 
Low-loss optical fibers are fabricated through glass-drawing methods resulting in extremely long fibers with consistent optical properties and making them ideal for longhaul communication. A doped preform is heated at the end while the molten glass is pulled at a controlled rate to maintain desired fiber dimensions. Backed by strong industrial interest optical fiber fabrication has become a mature technology that is affordable and commercially available for a variety of applications.

One major disadvantage in optical interconnects is the alignment costs and difficulties required to minimize coupling loss. Optoelectronic transceivers, typically laser diodes (LDs), exhibit an emission pattern that is elliptical with a wide angular divergence profile [5]. Direct contact connectorization results in poor LD-to-fiber coupling. The optical signals need to be corrected, focused, and aligned with the end of individual optical fiber bundles using lenses or microspheres for efficient coupling [6]. Alignment tolerances that need to be addressed in industrial applications that prefer passive connectorization are proportional to the optical fiber's dimensions.

Alignment costs limit optical fibers from being economically feasible for short-haul communications, including board-to-board and chip-to-chip interconnects. The fabrication costs to individually embed and align each optical fiber to transceivers are significantly higher than the price of optical fibers. The manufacturing time and total device cost is dependent on the quantity of required optical transmission lines and will become cost-inefficient as the number of required optical interconnects increases. Alternative optical interconnects are required to reduce manufacturing costs of short-haul 
optical routing, improve interconnect miniaturization, and eliminate off-board loose fibers.

\subsubsection{Rectangular Waveguides}

A cost-effective alternative to improve commercial feasibility of short-haul optical interconnects is through the implementation and integration of rectangular WGs within PCBs. An optical wiring board consisting of numerous $(>100)$ rectangular WGs can be fabricated simultaneously at specified dimensions and pitches through PCB-compatible manufacturing techniques. Rectangular WGs can compress multiple copper layers into a single optical layer that can then be integrated within the PCB. Embedded rectangular WGs further isolate the optical interconnects from the environment and reduce spatial requirements in contrast to over-hanging optical fibers that require manual connectorization. Interconnect buses of rectangular WGs can be connectorized simultaneously to surface-mount optoelectronic devices with specified pitches connecting multiple optical interconnects simultaneously. Out-of-plane coupling with surface-mount devices can be conducted through either internal mirrors [7] or embedded light-turning devices [8]. These factors reduce connectorization costs for rectangular WG-based optical interconnects. The connectorization process for rectangular WGs has been done with both active and passive procedures $[8,9]$.

Rectangular WGs have been designed using a variety of materials, including metal [10], glass [11], and polymers [12]. In the interest of commercial feasibility, polymer-based rectangular WGs are preferred for their low costs, ease in fabrication, environmental 
stability, and coupling and high manufacturing high-throughput. The elasticity of polymer materials can be engineered to remove material cracking and warping upon curing, allowing for the design of flexible [13] and stretchable [14] optical interconnects. The fabrication of polymer rectangular WGs can be also be integrated into many PCBcompatible techniques including direct (imprint lithography) [15] and indirect (photolithography) [16] imprinting methods. Polymer optical WGs fabricated from photo-lithography on a PCB dielectric substrate are shown in Figure 1.3.

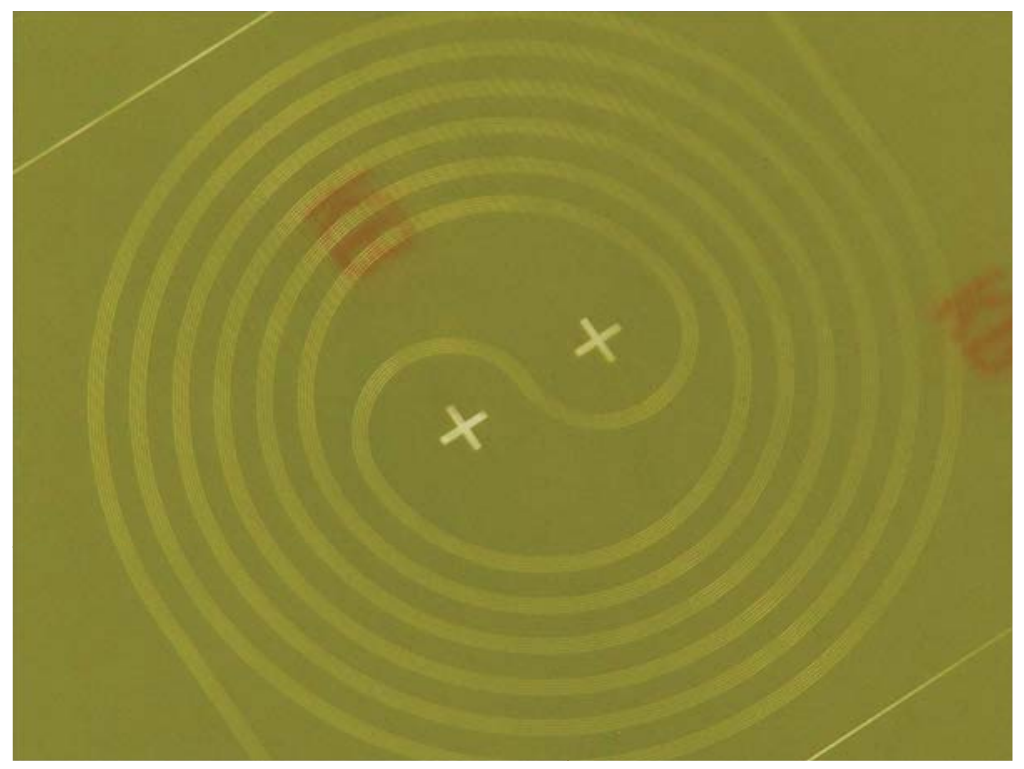

Figure 1.3: Optical interconnect bus of six polymer waveguides in the form of a 1 meter spiral are fabricated on PCB dielectric substrate.

The fabrication and integration of polymer rectangular WGs still requires advancements in research and development to become a commercially viable product. WG fabrication methods for both prototyping and mass production need to demonstrate low propagation and coupling loss for commercial feasibility. Advanced WG builds including WG bends 
and crossings should also exhibit low structure loss to optimize optical interconnect builds for minimum device losses and size requirements.

\subsubsection{Silicon Photonics}

Silicon ( $\mathrm{Si}$ ) photonics is an emerging technology enabling high-speed optical modulation [17] and lab-on-a-chip [18] devices feasible with large-scale CMOS integration [19]. Utilizing a silicon WG core $(n \sim 3.5)$ surrounded by a silicon dioxide cladding $(n \sim 1.5)$, high-density interconnects of sub-micron WG dimensions are possible with tight $(\mathrm{R}<5$ $\mu \mathrm{m}) \mathrm{WG}$ bend radii. Silicon photonic interconnects can achieve substantially higher compaction rates and tighter radial bends. Silicon photonic waveguide fabrication is conducted through CMOS-compatible manufacturing and integration into computer processing units (CPUs). Due to their high propagation loss $(>1 \mathrm{~dB} / \mathrm{cm})$ power budgets limit their applications to on-chip interconnects and active photonic chip devices.

\subsection{Research Outline}

Rectangular polymer WGs hereafter referred to as polymer WGs are cost-efficient interconnects for short-haul (board-to-board and chip-to-chip) applications. For successful integration polymer WGs must exhibit both low device loss and high coupling efficiency with silicon WGs and optical fibers for short-haul (on-chip) and long-haul (rack-to-rack) applications respectively. The main focus of the author's research was the design of a novel all-optical bridge module, as shown in Figure 1.4. The bridge module consists of polymer WG tapers and S-bends for optical coupling, mode conversion, and 
pitch control for efficient coupling between optical fibers and Si WGs. To completely understand the bridge module three main goals had to be achieved. The successful fabrication of low-loss polymer WGs needed to be realized using commercialy viable polymer materials. The theoretical understanding of fabricated WG S-bends were required for optimal WG pitch control with minimum footprint requirements. Polymer WG tapers required theoretical and experimental evaluation for efficient optical coupling and mode conversion for device connectorization applications.

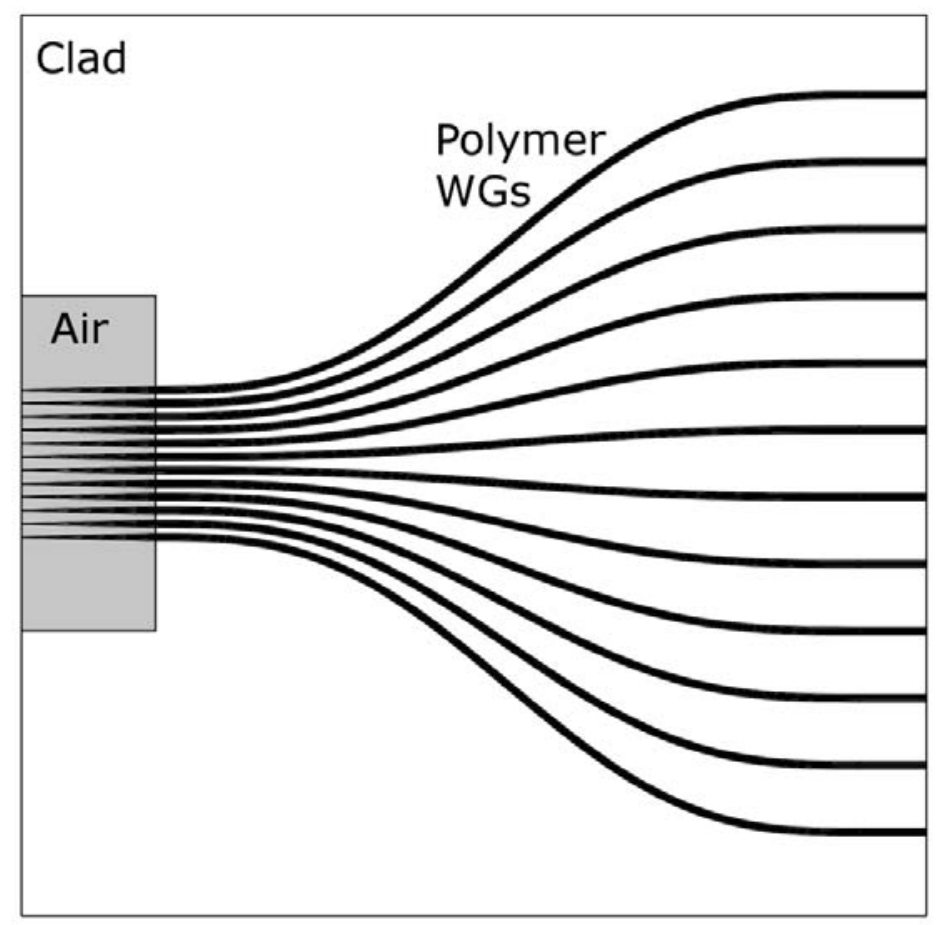

Figure 1.4 Polymer taper bridge module illustration.

This dissertation details the results in achieving these goals to advance the commercial feasibility of polymer WGs in improving short-haul interconnect bandwidth through the design, modeling, fabrication, and testing of polymer WGs and structures. Theoretical 
models and rigorous simulations are discussed in Chapter 2 and utilized to understand and optimize structure loss for polymer WGs of various designs and dimensions.

Chapters 3 and 4 detail the fabrication and experimentation of polymer WG structures utilizing UV-curable polymer materials with the photolithographic method and the LDW method as cost-effective methods for mass production and prototyping polymer WG builds respectively. Photolithography is the traditional, PCB-compatible method of polymer WG manufacturing due to high fabrication speeds but is financially-limited due to high-resolution mask costs. Laser Direct Writing (LDW) is an experimental, mask-less fabrication procedure for manufacturing large (>12") panel polymer WG builds. The LDW method has been previously reported in academia as method for fabricating polymer WGs [20, 21]. The LDW method has also been modeled to correlate writing parameters to the fabricated dimensions of Gaussian-shaped polymer WGs [22, 23]. The LDW method has neither been shown as a commercially viable method for WG fabrication nor has it been experimentally compared to photolithographic polymer WG builds.

The work presented in this dissertation summarizes the author's development of a new LDW model and process that produces consistent low loss polymer WGs of rectangular dimensions with high fabrication through-put. A theoretical model was derived and utilized to correlate polymer WG dimensions to multiple writing parameters. Optimal manufacturing parameters were obtained for the LDW of symmetric low-loss multi-mode $(0.045 \mathrm{~dB} / \mathrm{cm})$ and single mode $(0.55 \mathrm{~dB} / \mathrm{cm})$ polymer WGs. LDW straights, radial 
bends, and crossings do not exhibit significant deviations from photolithographicmanufactured WG device loss. Results validate the LDW method as an affordable polymer WG prototyping method before manufactures highly invest in large-scale photolithography for mass-manufacturing.

Polymer WG S-bends and tapers are discussed in Chapters 5. SM Polymer WG S-bends are required for optimal WG builds that include fan-out routing techniques for WG pitch control and optical splitters for power distribution and integrated interferometers. In high density optical interconnects the trade-off between S-bend structure loss and minimal footprint size must be understood to minimize optical link loss. Optical losses of sinederived S-Bends have been previously reported in academia with minimal losses, but never in the form of step-index polymer WGs. Previous literature reports proposed solutions for minimizing optical losses while obtaining high optical density with SM WG bend structures by utilizing polyline-based S-Bends [24] and radial tapers [25] to optimize WG designs. However, these complicated structures can be difficult to design and scale for integration into photolithographic masks. Furthermore, various publications $[26,27]$ have shown that sine-derived S-bends of equivalent dimensions offer similar levels of loss in simple, flexible, and expandable formulas. Semiconductor-based devices have been fabricated through MBE-grown epilayer [28] and etching [29]. Titaniumdoped Lithium Niobate S-bends were fabricated, tested, and correlated to theoretical losses [30, 31]. Experimental results for polymer-based SM WG S-bends have only been reported using the radial arc design with an emphasis on cross-talk measurements [32]. To the author's best knowledge, the author's work is the first to fabricate, evaluate, and 
compare S-bend designs utilizing step-index polymer SM WGs with an emphasis on minimizing device size and optical loss.

Complete structural loss analysis is determined theoretically and shown experimentally utilizing both radial loss and transitional loss in SM polymer WG radial-arc, cosine, and raised-sine S-bend profiles. Sinusoidal S-bends cannot be directly implemented into LDW and must be indirectly written through linear segmentation for compatibility with the platform's interface. LDW SM polymer WG S-bends were fabricated through the segmentation method, researched to obtain optimum manufacturing parameters, and measured to compare structure loss to photolithographic builds. Cosine S-bends were experimentally shown to exhibit the lowest loss for both photolithographic and LDW builds. Optical splitters designed with S-bends and tapers were also fabricated and measured showing that WG tapers reduce both length requirements and splitting loss. Splitters incorporating polymer tapers exhibit higher splitting efficiencies while minimizing splitter size.

Polymer WG structures can be altered to control optical confinement and improve coupling between various optical sources. Polymer WG tapers are used in Chapter 6 as a device for adiabatically transforming mode profiles to minimize coupling loss in photonic interconnect devices. Polymer WG tapers can be incorporated as a bridge module between high-density silicon ( $\mathrm{Si}$ ) photonic interconnects and long-haul optical fibers to improve optical coupling efficiency. Many authors have demonstrated success in improving device coupling through Si WG tapers $[33,34]$ and lensed SMFs $[35,36]$. 
However, it is advantageous to engineer an off-chip bridge module in a commercially viable package to improve optical coupling without incurring the requirements of costintensive end-face preparation methods for either Si WGs or SMFs.

In this dissertation novel coupling device taper structures are proposed as an off-chip coupling solution for short-haul optical interconnects. Vertical and multi-layer taper designs are proposed as cost-efficient optical coupling devices utilizing a metallic undercladding and an air/vacuum over-cladding to obtain strong modal confinement for high mode overlap with Si WGs. Simulation work on both mode conversion devices are conducted and discussed. Coupling efficiency is correlated to device dimensions, manufacturing constraints, and alignment tolerances. Tapers are fabricated with photolithography and measured to correlate device loss to metallic absorption and sidewall roughness. Fabricated polymer tapers were evaluated for coupling efficiency between WG tapers and Silicon WGs with high compatibility to theoretical coupling loss including mode overlap and interface reflection. 


\section{Chapter 2: Polymer Waveguide Theory}

\subsection{Introduction}

Optical confinement and guidance in optical interconnects is determined through boundary conditions based on electromagnetic (EM) phenomenon of total internal reflection to contain and guide optical signals. Approximations in EM propagation can be utilized to estimate device functionality with reduced computational resources. However these approximations have theoretical limitations in estimating device performance for optical interconnects. It is essential to utilize appropriate theoretical models and simulation methods in designing polymer WGs structures to meet engineering requirements including signal dispersion and signal-to-noise ratios.

This Chapter lays out a brief overview of the theoretical models and simulation techniques utilized to effectively model optical interconnects. The ray tracing method and EM modeling method for evaluating dielectric WGs are discussed and compared. These models are utilized to analyze theoretical losses including radial and transitional loss for advanced WG structures. Optical signals dispersion is also covered in this Chapter along with its causes and how it is measured. Simulation methods for the electromagnetic model are then described and compared for efficiently acquiring information needed to accurately model polymer WGs. 


\subsection{Propagation Theory}

\subsubsection{Ray Tracing Method}

The ray tracing method utilizes the directional properties of light to simulate optical propagation in a model as a summation of vectors. An optical system can be analyzed and optimized by sending multiple input vectors, all describing the optical source of interest, through the device and analyzing the output vector wavefront. This vector approximation can turn a complex differential equation-based EM problem into a simpler linear algebra problem.

When a ray of light is incident on a boundary at an angle $\theta_{l}$ between two different materials a fraction of the ray's energy will reflect off the boundary at $-\theta_{l}$ while the rest of the beam will propagate through the boundary at a refraction angle $\theta_{2}$. The refraction angle, which is dependent on the materials' refractive index (RI) values, $n_{l}$ and $n_{2}$, and the ray's incident angle $\theta_{1}$ is calculated according to Snell's Law shown in Eq. (2.1).

$$
n_{1} \sin \theta_{1}=n_{2} \sin \theta_{2}
$$

When a light ray in medium 1 is incident at the boundary where $n_{1}>n_{2}$ a specific incidence angle known as the critical angle, $\theta_{1}=\theta_{c}$, results in $\theta_{2}=90^{\circ}$. At the critical angle no power propagates into the second medium, a concept known as total internal reflection, at any angle $>\theta_{c}$, the critical angle shown graphically in Figure 2.1. During total internal reflection power is present in the second medium as a stationary, exponentially decaying field known as the evanescent field. 

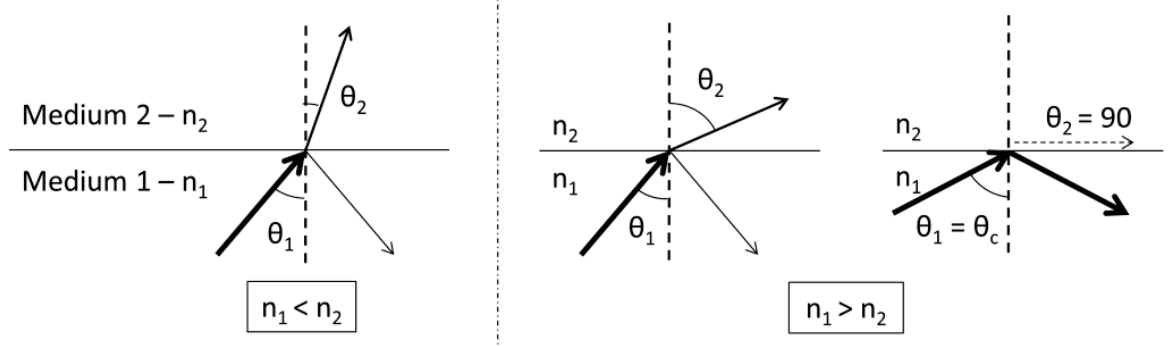

Figure 2.1: Correlation of incident and refracted angles with different boundary conditions.

By surrounding a 2 dimensional strip of high RI medium (WG core) with a material of lower RI (WG clad) the optical power can be contained in the strip indefinitely as long as the optical beam propagates while maintaining an angle of incidence at $\theta_{c}$. This is the general theory behind signal propagation in dielectric WGs. This effect is shown in Figure 2.2.

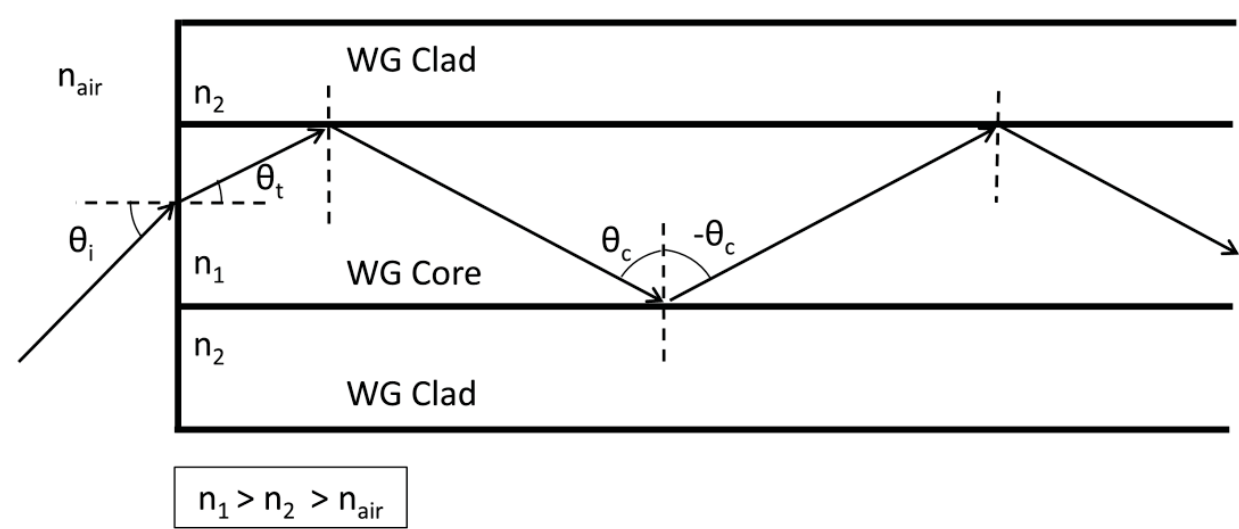

Figure 2.2: An illustration showing a ray tracing of a guided ray at the critical angle within a dielectric $W G$. 


\subsubsection{Electromagnetic Model}

While the ray tracing method is simple to visualize and utilize it is an approximate method and applicable only when modeling optical devices that are significantly larger (> $10 \lambda)$ than the wavelength of operation. Optical power propagates as a linear summation of time-varying waves specified by their wavelengths and the electromagnetic properties of the surrounding medium. The resulting field distribution within the WG can be solved for utilizing Maxwell's equations.

For optical propagation in dielectric WGs the environment will be absent of electrical charge, $\rho=0$, and electrical current, $J=0$. The material is also assumed to be isotropic. Taking these into consideration, Maxwell's equations can be expressed in their simplified forms shown in Eq. (2.2) - (2.5). $\vec{E}$ and $\vec{H}$ are the E-fiend and $\mathrm{H}$-field of the optical wave and $\varepsilon$ and $\mu$ are the electric permittivity and magnetic permeability of the surrounding medium respectively.

$$
\begin{gathered}
\nabla \cdot \vec{E}=0 \\
\nabla \cdot \vec{H}=0 \\
\nabla \times \vec{E}=-\mu \frac{\partial \vec{H}}{\partial t} \\
\nabla \times \vec{H}=\varepsilon \frac{\partial \vec{E}}{\partial t}
\end{gathered}
$$


The coherent signal will undergo interference with itself when confined in a dielectric WG and must satisfy specific internal reflection angles to obtain a state of selfconsistency. Every reflection angle that fulfills self-consistency allows for the propagation of a specific mode of order $m$ through the WG that exhibits a unique field distribution perpendicular to the propagation direction. To satisfy Maxwell's equations and these boundary conditions the confined mode exhibits a continuous profile that exhibits evanescent waves that exponentially decay into the cladding. Illustrations of various mode profiles are observed in Figure 2.3. The field distribution of guided modes in a WG of width $w$ is described in Eq. (2.6)-(2.9), where $\theta_{m}, \beta_{m}$, and $\gamma_{m}$ are the modedependent bounce angles, propagation constants, and extinction coefficients respectively that satisfy self-consistency within the dielectric WG [37].

$$
\begin{aligned}
& E(x) \propto \begin{cases}\cos \left(2 \pi \frac{\sin \left(\theta_{m}\right)}{\lambda} x\right) & \text { if } x<\left|\frac{w}{2}\right| \text { and } m \text { is even } \\
\sin \left(2 \pi \frac{\sin \left(\theta_{m}\right)}{\lambda} x\right) & \text { if } x<\left|\frac{w}{2}\right| \text { and } m \text { is odd }\end{cases} \\
& E(x) \propto \begin{cases}\exp \left(-\gamma_{m} x\right) & \text { if } x>\frac{w}{2} \\
\exp \left(\gamma_{m} x\right) & \text { if } x<-\frac{w}{2}\end{cases} \\
& \theta_{m}=\cos ^{-1}\left(\frac{\beta_{m}}{n_{\text {core }} k_{0}}\right) \\
& \gamma_{m}=\beta_{m}-n_{\text {clad }}^{2} k_{0}^{2}
\end{aligned}
$$


WGs that exhibit both large dimensions and high RI contrasts allow for the propagation of multiple modes and are classified as multi-mode (MM) WGs. The modal profile of higher-order WG modes $(m>0)$ exhibits higher levels of overlap with the cladding as the mode order increases. Each mode within a WG travels at a different velocity due to varying levels of core/cladding interaction. The modal velocity is specified as an optical signal propagating through a material of an effective RI, $n_{\text {eff. }}$ The effective RI of the guided mode is always $n_{\text {core }}>n_{\text {eff }}>n_{\text {clad }}$.

\begin{tabular}{|c|c|c|c|c|}
\hline Clad & $\mathrm{TE}_{00}$ & $\mathrm{TE}_{01}$ & $\mathrm{TE}_{02}$ & $\mathrm{TE}_{03}$ \\
\hline Core & & & $S$ & - \\
\hline Clad & & & & \\
\hline
\end{tabular}

Figure 2.3: The first 4 propagation modes in a multi-mode dielectric slab $W G$.

When an optical pulse enters a MM WG the signal power will couple into multiple modes. The pulse will undergo spreading as the faster, lower-order modes outpace the higher-order, slower modes. This amount of pulse spreading and distortion increases as the optical signal travels longer distances in the WG. This phenomenon is known as modal dispersion and is the biggest factor in limiting transmission speeds for optical WGs.

Single mode (SM) WGs are a special case where only one mode is guided in the dielectric WGs. By reducing both the WG size and RI contrast only the fundamental $\mathrm{T}_{00}$ 
mode, also known as the transverse electromagnetic mode (TEM), can propagate within the WG and modal dispersion is eliminated, as illustrated in Figure 2.4. Successful fabrication of SM WGs is a key ingredient in realizing high-speed optical interconnects in future devices, since larger MM WGs are bandwidth-limited due to modal dispersion. SM functionality is also a requirement for WDM in order to maintain a consistent intensity distribution along the WG length for ease in separating optical signals as a function of wavelength.

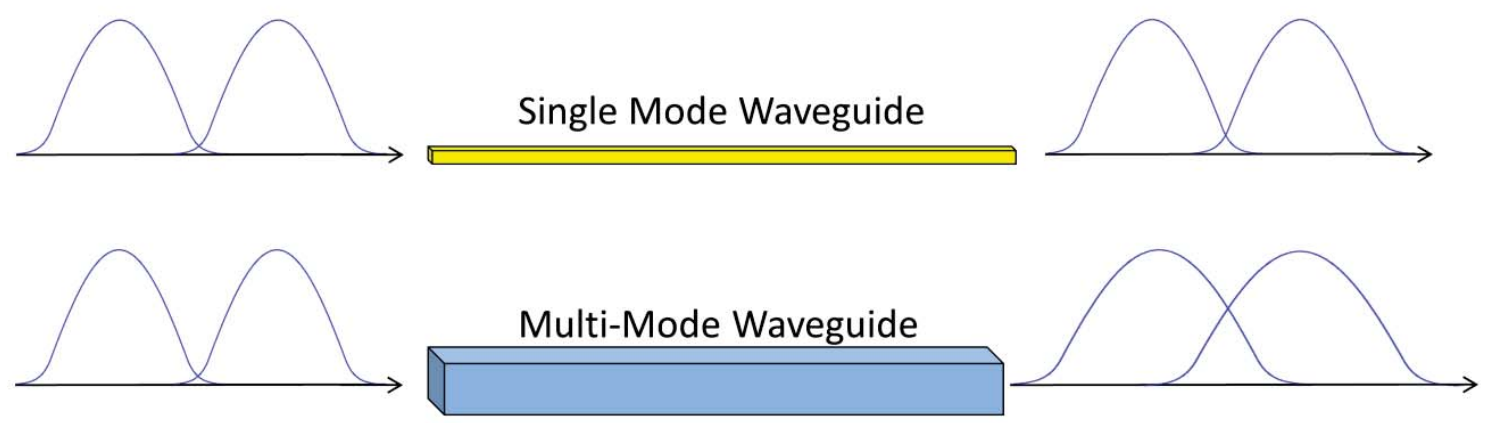

Figure 2.4: Signal Dispersion in a SM (top) and MM (bottom) Fiber.

\subsection{Propagation Loss}

While ideal dielectric WG straights theoretically exhibit no propagation loss physical WG builds will experience optical loss from both the material's intrinsic properties and boundary imperfections. Material loss is due to interaction between the signal photons and the atomic structure and/or impurities/imperfections within the dielectric medium. Surface loss is due to irregularities at the core/cladding interface. Losses are dependent 
on the WG material and the fabrication process used for WG manufacturing and need to be optimized in the fabrication of low loss optical interconnects. The various loss components are briefly discussed below.

\subsubsection{Material Loss}

Molecular bonds absorb infrared (IR) energy before being transferred to vibrational (thermal) energy. Energy absorption as a function of wavelength is known as the material's absorption spectrum. Polymers are designed by substituting specific functional groups with more inert chemical bonds and reduce the amount of optical attenuation at the wavelengths of operation. As an example, $\mathrm{C}-\mathrm{H}$ bonds are known to exhibit high absorption levels in telecom wavelength $(\lambda=1300-1600 \mathrm{~nm})$ [38]. C-H bonds can be substituted in polymer materials with C-F bonds to lower absorption levels in the IR [39], but polymer fluoridation impedes its feasibility as a WG material due to limited solubility, reduced adhesion, and increased toxicity $[39,40]$.

Polymer materials also exhibit volume scattering. As an amorphous material the atoms in the medium do not form a crystalline lattice and exhibit density fluctuations, voids, and contaminants causing fluctuations in the material's RI and act as scattering regions within the polymer medium, adding to the WG's propagation loss [41].

\subsubsection{Surface Loss}

Optical signals experience surface scattering loss from fabrication imperfections at the core/cladding boundary. Random fluctuations in the WG's width result in ray scattering, 
mode-mismatch, and pseudo-grating effects that attenuate the propagating optical signal. Higher-order propagating modes attenuate faster from structural loss as they experience more reflections at the WG boundary.

The major source of surface scattering loss is WG sidewall roughness [42] that varies by the method of WG fabrication. WGs exhibit sidewall roughness from mask roughness imprinted through photolithography. Material removal methods, including laser ablation and reactive ion etching, incur high roughness levels unless the process is finely tuned to minimize sidewall damage [39].

Other general processing parameters will cause fluctuations and micro-bends in the WG structure. A spin-coated core layer will experience slight vertical fluctuations on thickness based on the materials spin properties and the roughness profile of the substrate and cladding layers underneath. Physical and thermally-induced stresses from material shrinkage, curing temperatures, and mismatch of thermal expansion characteristics between core/cladding material resulting in micro-bending and localized deformations in the WG [5].

\subsection{Geometric Structure Loss}

Optical interconnects require advanced WG structures, including bends and crossings, for practical builds. Structure loss from these advanced structures can be decomposed into length-dependent radial loss and junction-localized transitional loss. For example, curved

dielectric WGs exhibit both forms of loss, as shown in Figure 2.5. Radial loss [43-45] is the 
result of continuous power radiation along the entire WG bend due to the asymmetric shift of the guided mode into the caustic region. Transitional loss $[46,47]$ is the result of coupling loss that arises from modal mismatch at straight/bend interfaces. Furthermore, transitional loss can occur both within WG structures or when coupling between two separate devices with mismatching modal field distributions. Both loss mechanisms have been thoroughly studied in the previous literature and established as complex functions of the WG's physical properties. Both loss types are complex functions of the WG's physical properties and will be discussed in brief.

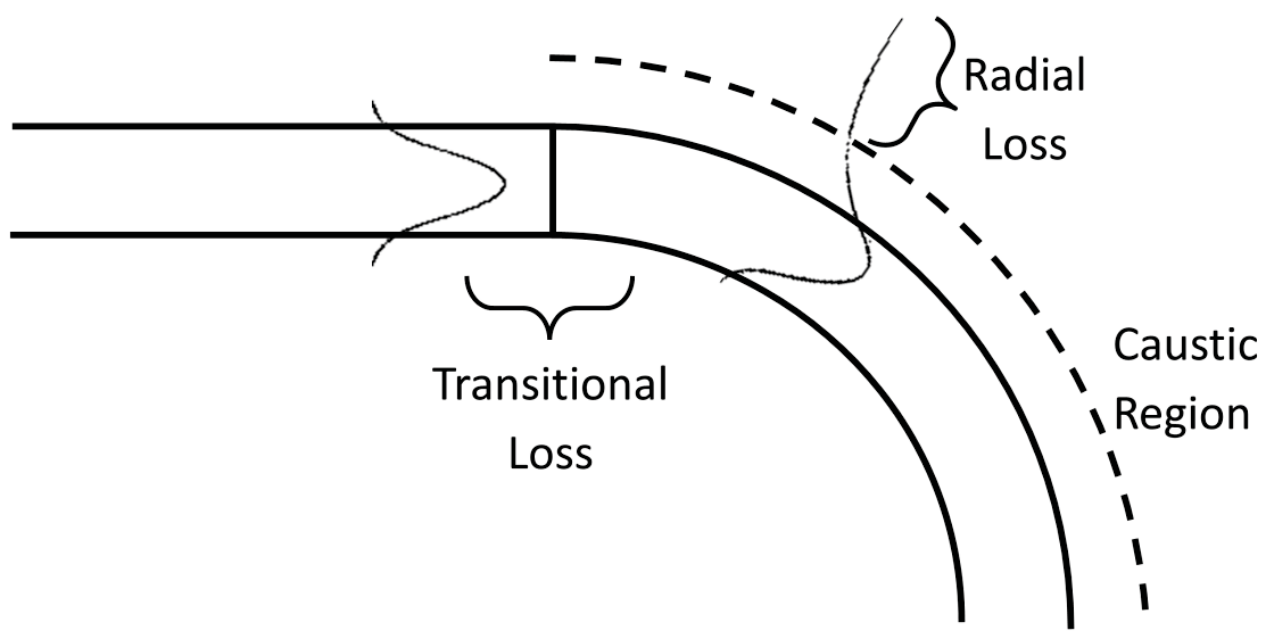

Figure 2.5: Transitional and Radial Losses of a propagating mode in a WG Bend.

\subsubsection{Radial Loss}

Radial loss is based on the theory of limited guidance of the mode due to speed limitations of the mode traveling on the outer end of the bend. As a mode propagates through a WG bend the mode elongates and shifts to the outer edge of the WG. This 
radially propagating mode experiences a velocity gradient that increases from the radius's center. This can be modeled as a straight WG with a RI gradient $n(x)$ which decreases towards the outside of the bend. The outer region of the mode beyond the caustic region, where $n(x)<1$, cannot propagate faster than the speed of light and is no longer guided radiating towards the outside of the bend. The rate of radiation loss is dependent on the amount of overlap the mode exhibits in the caustic region.

Boundary conditions require modal profiles in tighter WG bends to shift and extend farther into the caustic region. Radial loss increases with decreasing WG bend radii, $R$. As shown by Marcatili [48], the radial attenuation coefficient, $\alpha(R, s)$, a guided mode exhibits per unit length, $\mathrm{s}$, in a bend of constant radius, $S$, is expressed by Eq. (2.10). $C_{1}$ and $C_{2}$ are radially-independent loss coefficients that are dependent on the WG's NA and size $[31,48]$.

$$
\alpha(R, s)=C_{1} \exp \left(-C_{2} R(s)\right)
$$

To determine the total radial loss, the radius of curvature function, $R(s)$, of the S-bend profile, $y(x)$, must first be known and is obtained using Eq. (2.11). By substituting Eq. (2.11) into Eq. (2.10), the normalized radial efficiency $\Gamma_{R}$, is determined by the integration of $\alpha(R, s)$ over the length of the S-Bend. The radial efficiency function is shown in Eq. (2.12), where $s^{\prime}$ and $S$ are the incremental and total length of the S-bend respectively [29]. 


$$
\begin{gathered}
R(s)=\frac{\left(1+y^{\prime}(x)^{2}\right)^{\frac{3}{2}}}{\left|y^{\prime \prime}(x)\right|} \\
\Gamma_{R}=\exp \left(-\int_{0}^{s} \alpha\left(R\left(s^{\prime}\right), s^{\prime}\right) d s^{\prime}\right)
\end{gathered}
$$

\subsubsection{Transition Loss}

Transitional loss arises when a discontinuity in the WG's modal profile occurs between two optical devices resulting in reduced coupling efficiency. Transitional loss can arise from multiple causes, including NA mismatch, mode order mismatch, beam divergence, and device misalignment. The normalized transitional efficiency, $\Gamma_{T}$, is determined by the overlap integral between the input and output electric fields, $E_{\text {in }}$ and $E_{\text {out }}$, as shown in Eq. (2.13) [49].

$$
\Gamma_{T}=\frac{\left|\iint E_{\text {in }}(x, y) E_{\text {out }}^{*}(x, y) d y d x\right|^{2}}{\iint\left|E_{\text {in }}(x, y)\right|^{2} d x d y \iint\left|E_{\text {out }}(x, y)\right|^{2} d x d y}
$$

An interesting example occurs in radial bends. Guided modes within curved WGs are wider and asymmetrically shifted towards the outside of the curve in comparison to straight WG modes. While Eq. (2.13) is the general form for determining transitional loss, simplifications in determining mode misalignment-based loss for radial bends has been discussed by various authors for both optical fibers [46, 47] and dielectric WGs [24]. 
While there is a slight size different between the straight and radial modes the main contributing factor to overlap mismatch is the modal displacement $\Delta_{x}$ that exists in the radial bends. As the WG's radius of curvature decreases the radial mode shifts away from the WG's centers as a function of wavelength, $\lambda$, WG width, $w$, and effective refractive index, $n_{\text {eff. }}$ Taking into account this modal shift in WG bends for symmetric WGs transitional loss has been analyzed and simplified by previous authors [46, 47]. These studies note an inverse quadratic relationship between bend radius and the resulting transitional loss in decibels at the straight-bend interface, as shown in Eq. (2.14).

$$
\Gamma_{T, d B}=T_{1} R^{-2}, \quad T_{1} \propto\left(\frac{\pi^{2} n_{e f f}^{2} w^{3}}{\lambda^{2}}\right)^{2}
$$

Transitional loss can be eliminated by incorporating additional WG segments to reduce discontinuities in the optical interconnects. Adiabatic tapers have been utilized as mode conversion devices to minimize coupling between devices of different dimensions [50]. Radial tapers can be placed between the straight/bend interface to create a gradual increase in the WG's curvature and eliminate any discontinuities in the bend's curvature [25]. However, both traditional and radial tapers require extra space for effective implementation increasing the required area for optimal performance.

\subsubsection{Total Structure Loss}

Optical interconnect designs will have multiple regions of transitional loss and radial loss. Radial losses occur wherever the radius of curvature is not infinite. Transitional 
losses are incorporated where there are discontinuities in the modal profiles. Assuming the individual normalized outputs are independent from one another the product of all the normalized outputs results in the total structure efficiency. Converting the losses in terms of $\mathrm{dBs}$ the total normalized loss of the structure is the summation of the normalized structure loss elements, as shown in Eq. (2.15). This will give the link power budget for individual dielectric WG in a theoretical optical interconnects build.

$$
\Gamma_{\text {Total }, d B}=10 \log _{10}\left(\prod \Gamma_{R} \prod \Gamma_{T}\right)=\sum \Gamma_{R, d B}+\sum \Gamma_{T, d B}
$$

\subsection{Dielectric WG Model and Simulation Methods}

While analytical solutions for 2D dielectric WGs exist to solve for polymer WG functionality the same does not hold for 3D structures. Hybridization occurs between the TE and TM propagation modes and analytical solutions for the propagating modes do not exist [49]. Determining mode profiles, loss coefficients, and mode cutoff requirements for 3D dielectric SM WGs have to be determined either indirectly with approximate solutions or directly through numerical methods.

\subsubsection{Effective Index Method}

The hybridization of the TE and TM modes in a 3D WG structure creates a modal profile that extends in all directions from the WG core into the cladding, as shown in Figure 2.6. Assuming that the mode has negligible presence in the corner regions of the cladding material, shown in Figure 2.7, the design can be broken down as two perpendicular 2D 
WGs. The modal profile can be approximately solved for as a slab WG with well-known solutions. This method has been thoroughly described by Marcatili [51] and improvements have been discussed by others $[52,53]$.

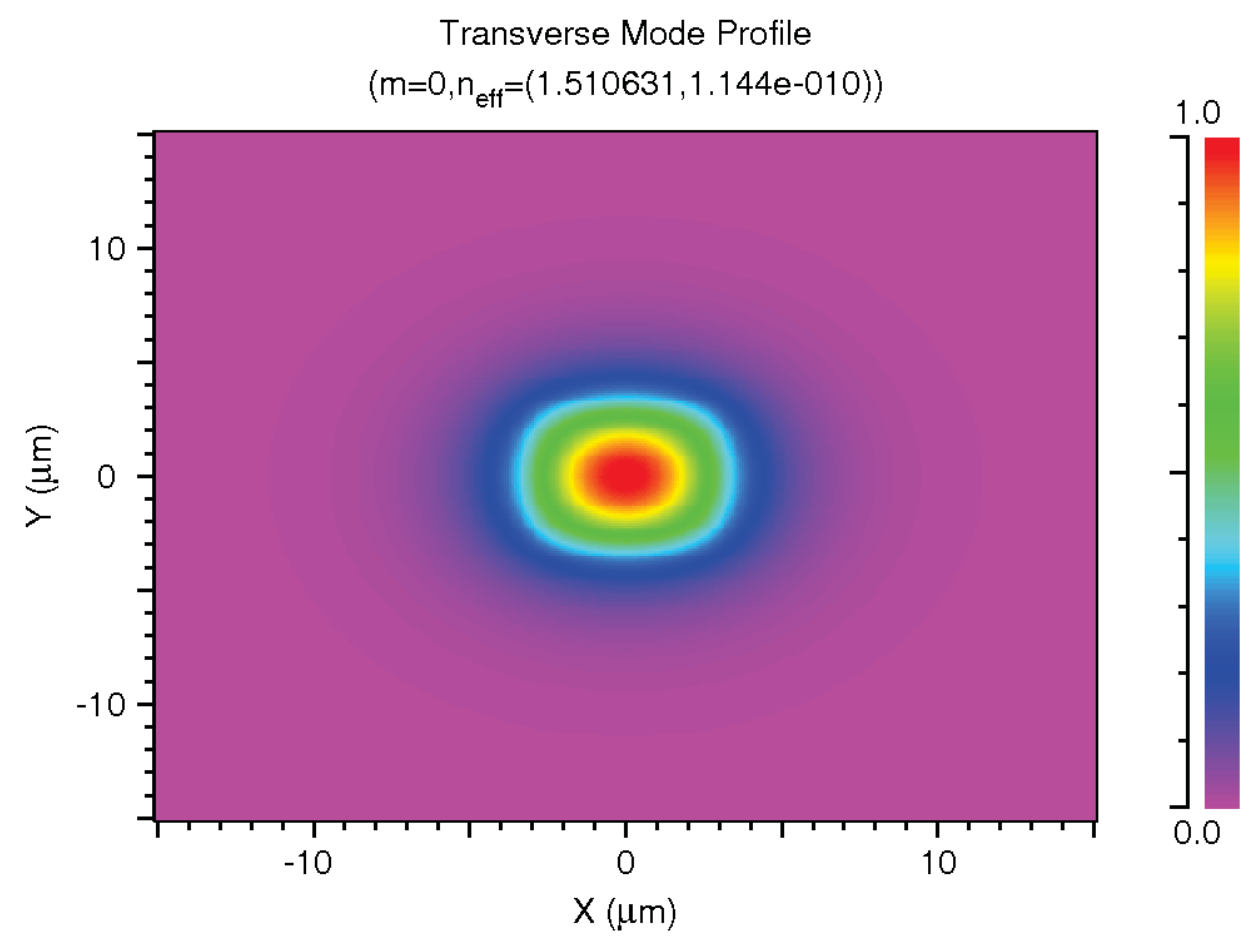

Figure 2.6: The TEM mode of a $6 \mu \mathrm{m}$ SM WG extends into the cladding in all directions. 


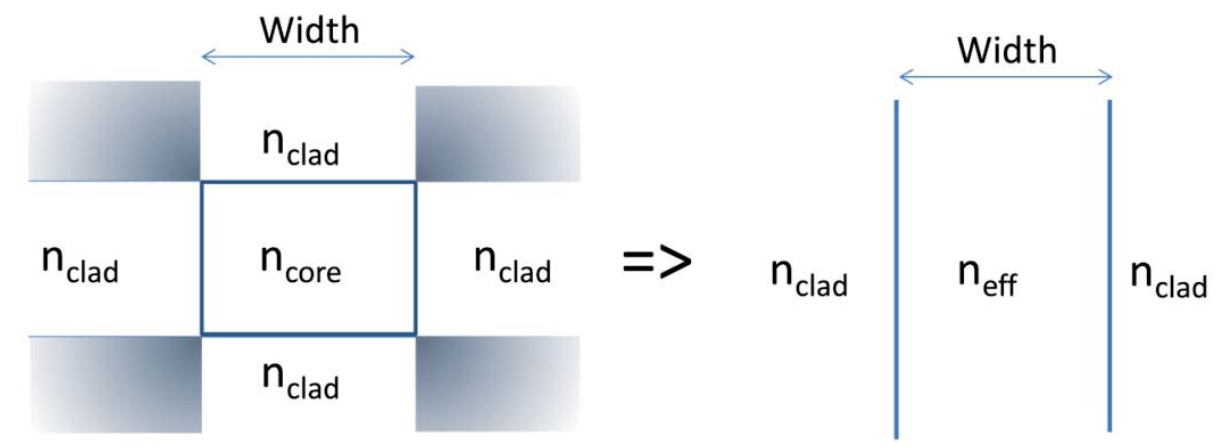

Figure 2.7: Assumes negligible modal presence in the shaded corners of the WG cross-section, the Effective Index Method [51] can be utilized to convert a 3D WG into a $2 D$ slab $W G$ for modal approximations.

\subsubsection{Finite Difference Time Domain}

The Finite Difference Time Domain (FDTD) is a direct solution to Maxwell's equations in Cartesian coordinates to solve for optical propagation through the optical system of interest $[54,55]$. Maxwell's curl equations, all exhibiting the same form shown in Eq.

(2.16), are utilized to solve for the electric and magnetic fields, $\vec{E}$ and $\vec{H}$, present in the 3D WG model. The FDTD method discretizes the model making it compatible for numerical evaluation, as shown in Figure 2.8.

$$
\frac{\partial H_{x}}{\partial t}=\frac{-1}{\mu}\left(\frac{\partial E_{y}}{\partial z}-\frac{\partial E_{z}}{\partial y}\right)
$$




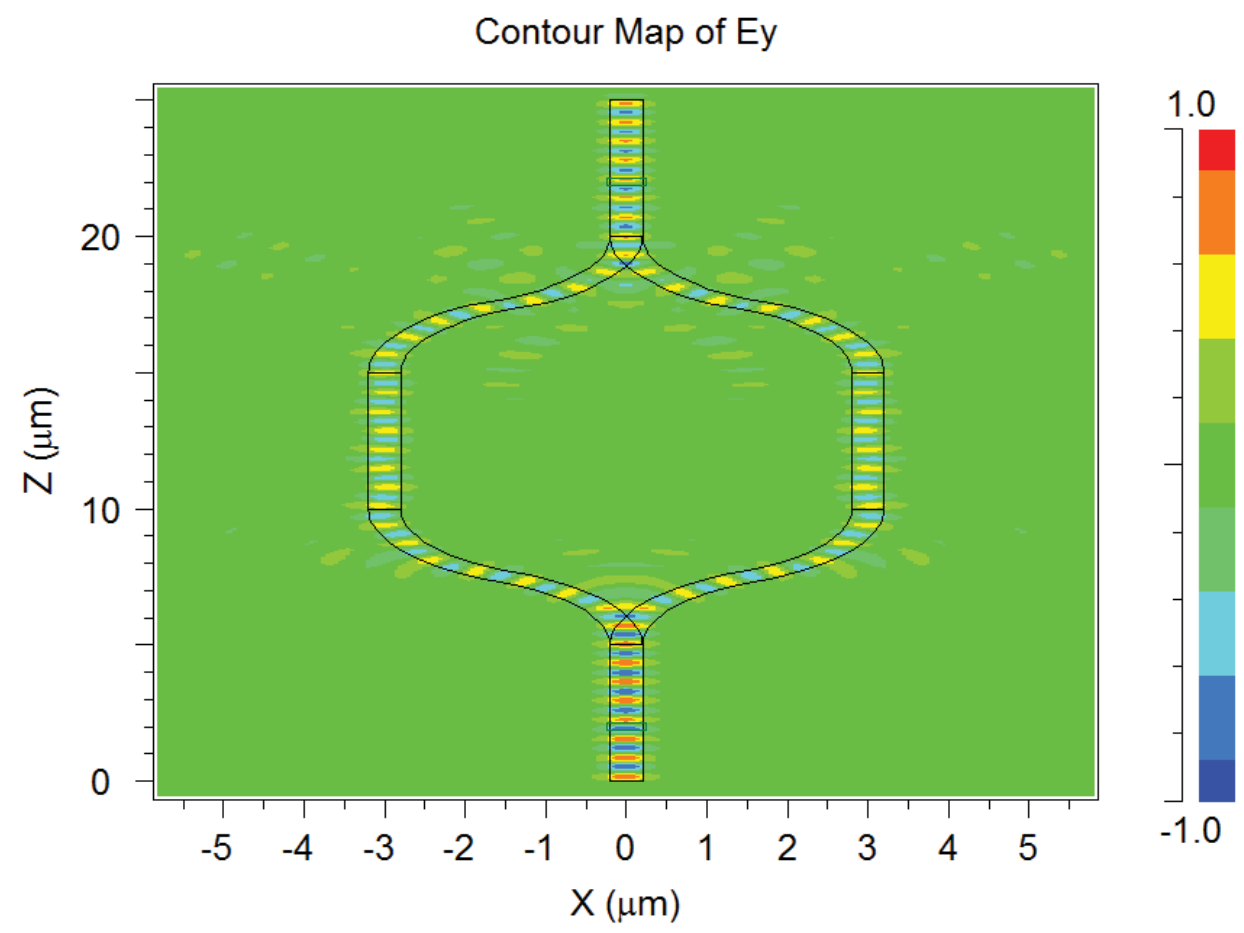

Figure 2.8: The FDTD method in simulating a Mach-Zehnder interferometer. The peaks and troughs of the electric field are visually observed during a discrete instance in time.

The accuracy of the simulation is determined by the resolution used for both spatial and temporal dimensions. Spatial grid size are recommended not to exceed $\Delta x, y, z=\frac{\lambda}{10 n}$ [55], where $n$ is the material's refractive index, to ensure that the optical wavelength is accurately resolved. Temporal resolution is usually required not to exceed the Courant condition [55] which is dependent on the spatial grid resolution $\Delta x, \Delta y$, and $\Delta z$ so the user can acquire a stable simulation.

The FDTD method is the most accurate method for solving for optical propagation, mode profiles, and device loss. As the most mathematically rigorous simulation available, the 
FDTD method is essential for simulating complicated optical designs. However, obtaining simulation results from the FDTD method is very intensive in terms of computing power and time requirements which are directly proportional to the size of the dielectric WG model.

\subsubsection{Beam Propagation Method}

The Beam Propagation Method (BPM) is another tool for determining optical propagation and confinement in many passive optical devices including fiber optics and dielectric WGs. By assuming that the optical propagation doesn't deviate significantly from the axis of propagation, $z$, Maxwell's equations can be significantly simplified. The overall solution, the Helmholtz equation shown in Eq. (2.17), is a steady-state model of the optical power in the medium. Being steady-state modeling optical propagation over time is non-essential significantly reducing computing time. Solving for optical confinement over propagation distance it can be assumed that the E-field is slowly varying in the $z$ direction and the Helmholtz equation can be reduced to the form shown in Eq. (2.18).

$$
\begin{gathered}
\nabla^{2} \vec{E}+k^{2} \vec{E}=0 \\
\frac{\partial \vec{E}}{\partial z}=\frac{j}{2 \beta}\left(\frac{\partial^{2} \vec{E}}{\partial x^{2}}+\frac{\partial^{2} \vec{E}}{\partial y^{2}}+\left(k^{2}-\beta^{2}\right) \vec{E}\right)
\end{gathered}
$$

In contrast to the FDTD method the BPM solves for the simplified Helmholtz equation by simply integrating along the propagation direction, $z$. The simulation is capable of 
determining confined steady-state optical power as it propagates through the model, as shown in Figure 2.9. While useful for straight optical components the BPM fails to accurately simulate devices where the optical power deviates significantly from the propagation axis. The BPM does not take into account the propagation of reflected light at boundaries since this method does not calculate negative traveling waves. As a result, optical devices that cannot be modeled through the BPM include highly divergent optical sources, radial bends, mirror reflections, and optical gratings. The simulation of WG bends and curves can be approximated using the BPM by utilizing a transverse coordinate transformation or conformal index mapping on a straight WG model.

\subsubsection{Mode Solvers}

To utilize the FDTD and BPM methods to solve for optical propagation the methods require a known initial state (the input field into the model). This initial state is typically the fundamental mode of the channel WG. By conducting a vector finite difference analysis along the 2D cross-section of a WG mode solvers can obtain all the possible mode structures and their effective RI values that are capable of optical confinement [56]. Mode solvers are also useful tools in determining WG properties required to eliminate MM excitation in the polymer WG. 

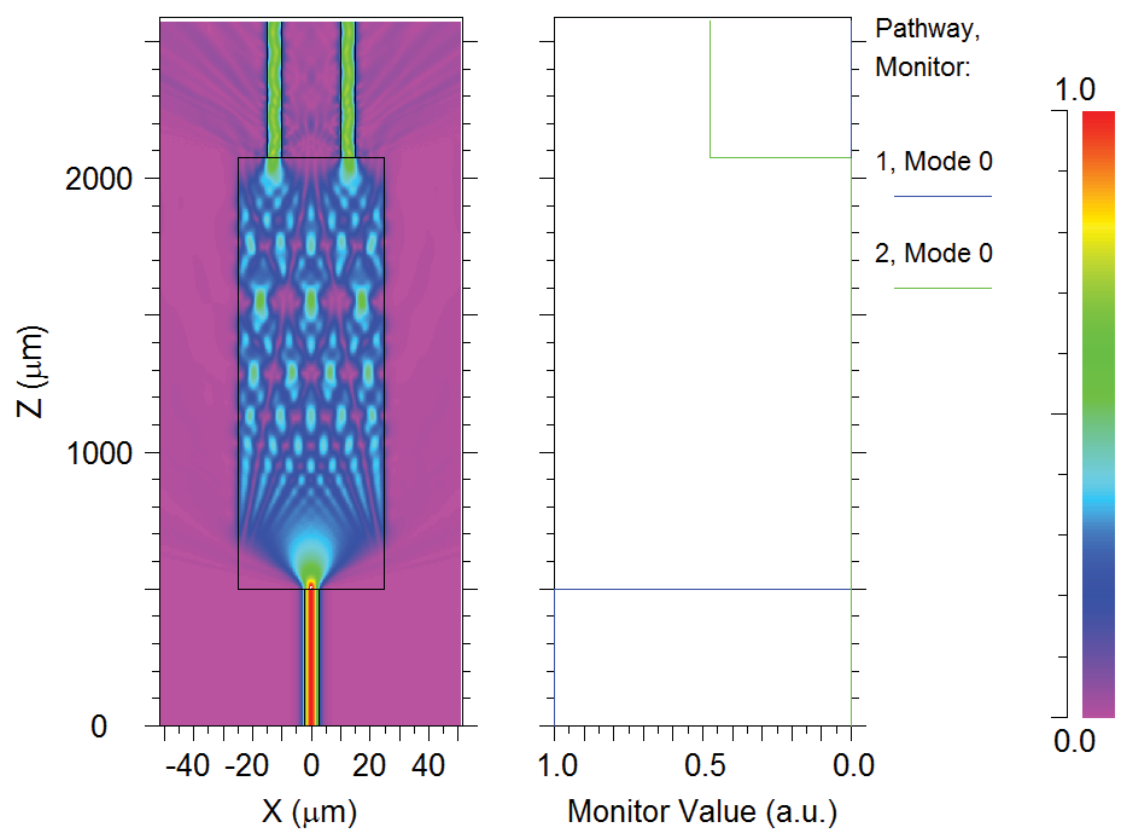

Figure 2.9: The BPM utilized to simulate a MM interference splitter. The propagation power is assumed steady-state.

By combining Maxwell's curl equations previously described in Eq. (2.16) the longitudinal field, $H_{z}$, is observed as a function of the transverse magnetic fields, $H_{x}$ and $H_{y}$, as shown in Eq. (2.19). With this simplification the coupled eigenvalues for the transverse magnetic fields can be solved for through vector analysis, as shown in Eq. (2.20), where $A_{i, j}$ are differential operators. The differential operators give the user information on the modes and their propagation constants.

$$
\begin{gathered}
H_{z}=\frac{1}{j \beta}\left(\frac{\partial H_{x}}{\partial x}-\frac{\partial H_{y}}{\partial y}\right) \\
{\left[\begin{array}{ll}
A_{x x} & A_{x y} \\
A_{y x} & A_{y y}
\end{array}\right]\left[\begin{array}{l}
H_{x} \\
H_{y}
\end{array}\right]=\beta^{2}\left[\begin{array}{l}
H_{x} \\
H_{y}
\end{array}\right]}
\end{gathered}
$$


Mode solvers can determine the number of propagating modes for a $3 \mathrm{D}$ dielectric $\mathrm{WG}$, as shown in Figure 2.10. As the WG structure has multiple eigenvalue solutions pertaining to both guided and radiation modes the solutions can be paired to mode order based on the magnitude of the solution. For an isotropic channel WG the fundamental mode will always have the highest $n_{\text {eff }}$ value. For every higher order mode one can determine the difference between guided and radiation modes based on its $n_{\text {eff. }}$ A mode is considered guided when $n_{\text {core }}>n_{\text {eff }}>n_{\text {clad }}$.
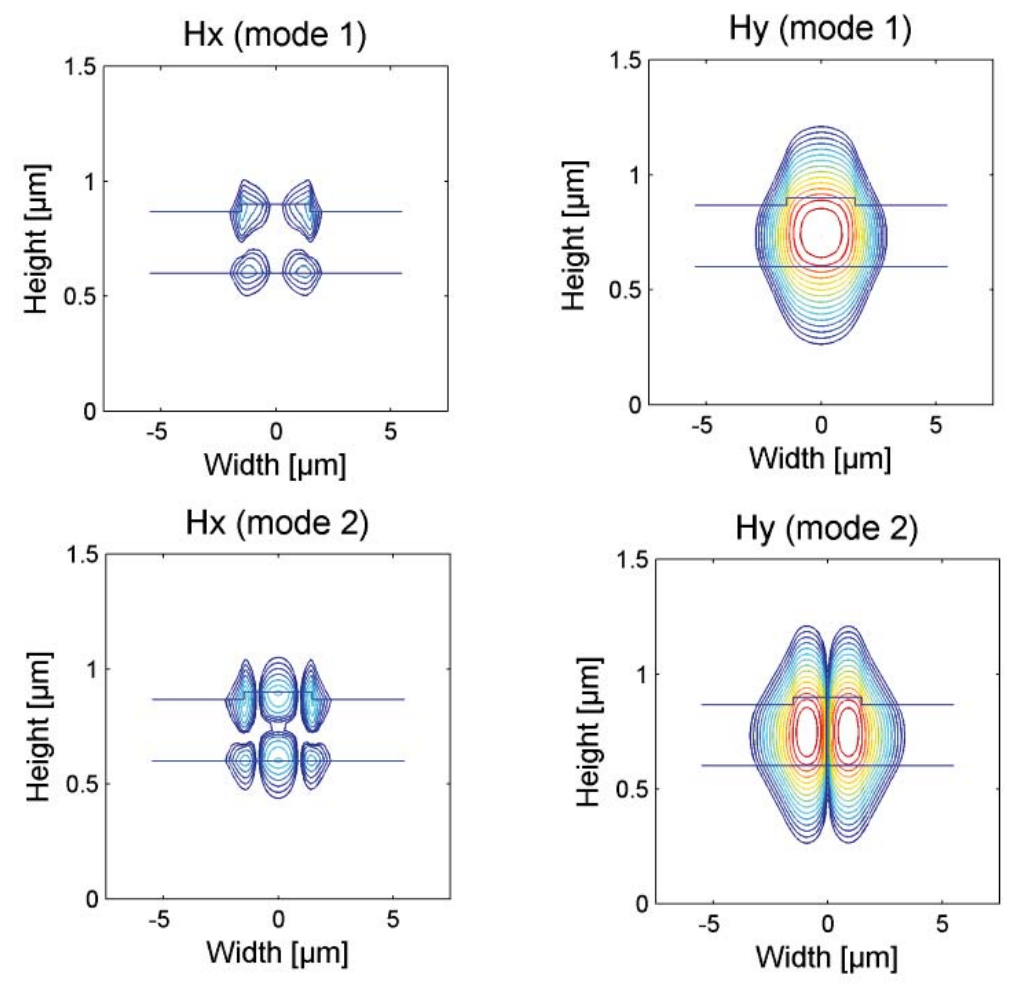

Figure 2.10: Modes for a partially-etched rib WG solved for using a finite difference mode solver, showing only modes with $H_{y}$ polarization being confined. 


\subsection{Conclusion}

Modal propagation in polymer WGs is based on Maxwell's equations in terms of optical coupling, modal confinement, and signal propagation. The parameters of polymer WGs can be controlled so they exhibit the desired characteristics of higher bandwidth limits. Three dimensional polymer WGs must utilize simulations to accurately predict WG performance. Mode solvers are initially required in determining both WG requirements for SM functionality and input field parameters required in simulating theoretical devices. Numerical (FDTD and BPM) simulation methods conduct theoretical optical propagation through a WG device to evaluate sources of loss and expected performance. 


\section{Chapter 3: Waveguide Fabrication and}

\section{Evaluation}

\subsection{Introduction}

Before successful commercial implementation of optical interconnects can be achieved cost-efficient procedures in the development of polymer WGs must be demonstrated for efficient manufacturing of low-loss, high-bandwidth optical interconnects. In this Chapter, the manufacturing and characterization procedures for polymer WGs are discussed. A suitable polymer material must be chosen with optimal optical properties, easy to fabricate, and environmentally stable. Evaluation of optical properties of materials is discussed using prism coupling and ellipsometry. Physical WG builds will exhibit length-dependent propagation loss, coupling efficiency, and structure imperfections, all which must be determined for overall link loss calculations for future builds. Polymer WG fabrication through photolithography is detailed and conducted. Finally, waveguide testing and evaluation procedures are laid out to isolate and quantify polymer WG loss.

1. Research in this chapter has been previously published by the author in the following reference:

- K. L. Kruse and C. T. Middlebrook, "Fan-out routing and optical splitting techniques for compact optical interconnects using single-mode polymer waveguides," Journal of Modern Optics, pp. 1-10, 2014. 


\subsection{Dielectric WG Materials}

Optical WGs can be manufactured using many different materials, such as metal [3], glass [11], and polymers [12]. While all these materials can be manufactured with low absorption coefficients at telecom wavelengths manufactured WGs must also be costeffective to fabricate and environment insensitive. Polymer-based WGs are the lowest cost and most flexible materials to utilize for the integration of optical interconnects into PCBs. Their elastic properties also minimize unwanted cracking in stressful environments. Furthermore, polymer materials can be engineered to become more resistant to high-temperature and high-humidity environments.

UV-curable optical elastomers from Dow Corning ${ }^{\circledR}$ were used as the core and cladding for this research. The polymers have been engineered to exhibit low losses at $\lambda=850 \mathrm{~nm}$ $(<0.02 \mathrm{~dB} / \mathrm{cm})$ and $\lambda=1310 \mathrm{~nm}(<0.4 \mathrm{~dB} / \mathrm{cm})$, as shown in Figure 3.1 [12]. Polymerization is initiated under broadband UV and driven to completion through thermal curing. They are also capable of resisting harsh environments including high temperatures and high humidity tests [12], are compatible with PCB processes $[9,57]$, and can be easily integrated in large-scale manufacturing [58].

The refractive index of the Dow Corning ${ }^{\circledR}$ UV-Cured Optical Elastomer was measured for the core $\left(\mathrm{n}_{\text {core }}=1.5142\right)$ and cladding $\left(\mathrm{n}_{\text {clad }}=1.5064\right)$ material respectively resulting in a theoretical NA $=0.135$ assuming true step-index polymer WGs. SM operation is feasible when the symmetric WGs are fabricated with dimensions of $6 \mu \mathrm{m} \times 6 \mu \mathrm{m}$. With dimensions and optical properties similar to most commercially-available SMFs these 
values ensure optimal coupling efficiency and alignment tolerance between SM WGs and SMFs.

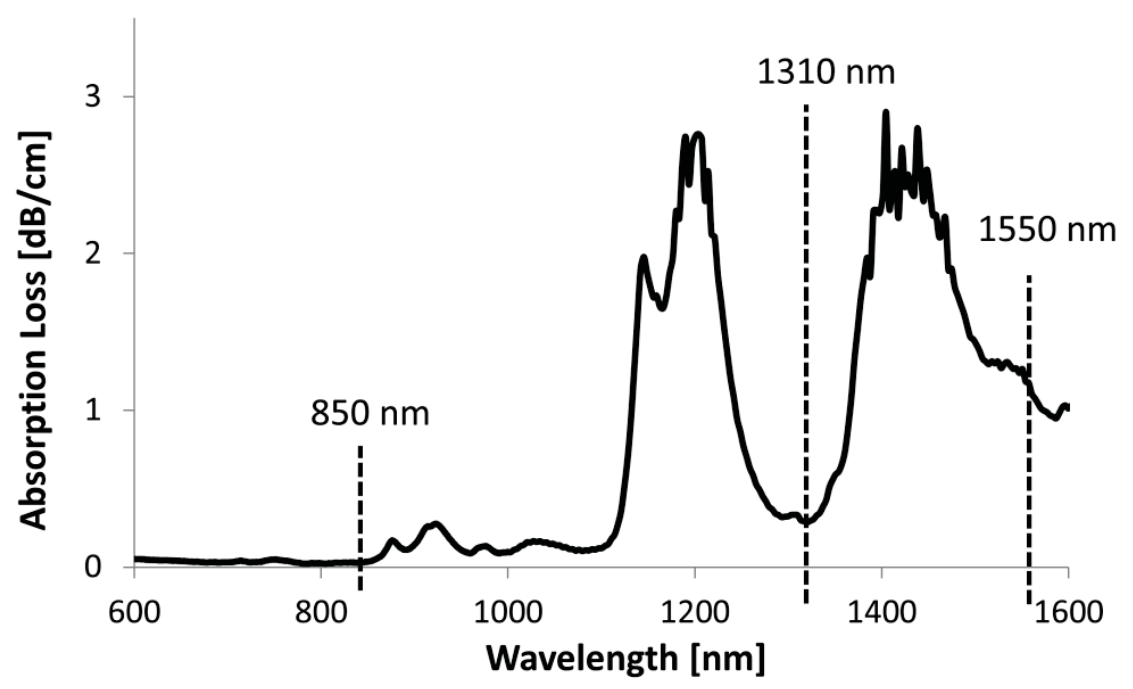

Figure 3.1: IR absorption spectrum of polymer WG core material.

\subsection{Fabrication Procedures}

SM polymer WGs were fabricated with the WG materials using the following procedure. A $20 \mu \mathrm{m}$ layer of cladding ( $25 \%$ solvent) was spin-coated (1000 RPM) on a cleaned substrate. The under cladding planarizes the optical interconnect plane to reduce unwanted surface roughness and isolate the optical signal from the absorbent substrate [12]. Polymer WG propagation loss has been experimentally shown to be independent of substrate type $[59,60]$. The cladding was then pre-baked $\left(110^{\circ} \mathrm{C}, \mathrm{t}=2 \mathrm{~min}\right), \mathrm{UV}$ cured $\left(1200 \mathrm{~mJ} / \mathrm{cm}^{2}\right)$, and post-baked $\left(110^{\circ} \mathrm{C}, \mathrm{t}=2 \mathrm{~min}\right)$ to polymerize the exposed material. A $6 \mu \mathrm{m}$ core layer of diluted elastomer (50\% solvent) was spin-coated (750 RPM) and then 
pre-baked $\left(110^{\circ} \mathrm{C}, \mathrm{t}=2 \mathrm{~min}\right)$ to remove unwanted residual solvent. The spin-coating speed for the appropriate film thickness was obtained by conducting a spin-curve profile, with the results shown in Figure 3.2.

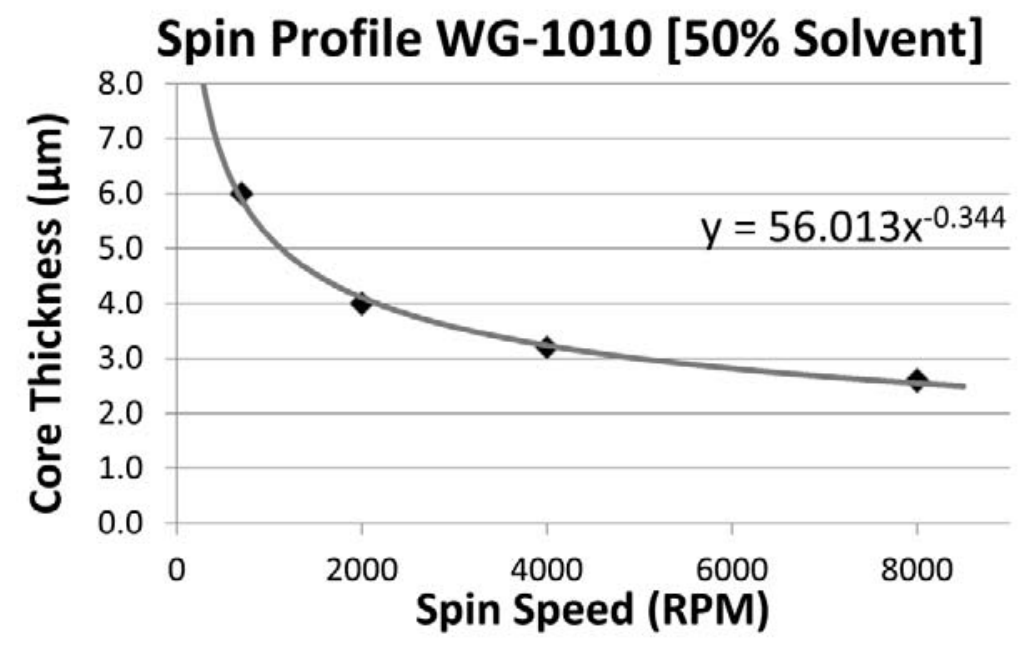

Figure 3.2: Spin Curve of UV-curable optical elastomer utilized in polymer $W G$ fabrication.

Polymer WG patterning can be conducted using a variety of PCB-compatible methods including photolithography. After appropriate UV initiation the exposed polymer is thermally polymerized and developed using appropriate organic solvents. The polymer WGs were hard-baked $\left(130^{\circ} \mathrm{C}, \mathrm{t}=24\right.$ hours $)$ to reduce material permeability before an over-cladding layer was applied accordingly. The fabrication procedure is outlined in Figure 3.3. 


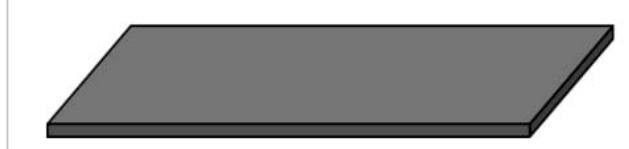

\section{1) Clean Substrate}

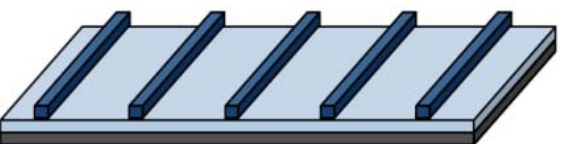

\section{3) Apply, Pattern, and Develop Core}

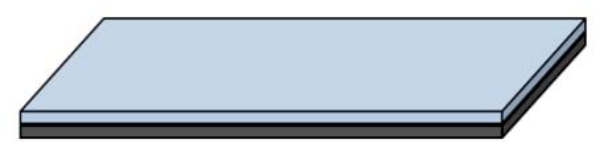

2) Apply and Cure Lower Clad Layer

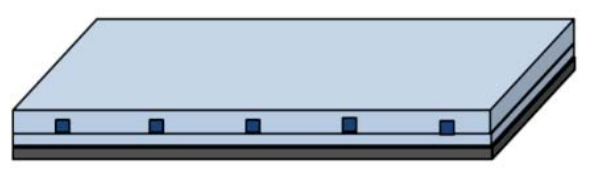

\section{4) Apply and Cure Top Clad}

Figure 3.3: WG fabrication process.

\subsubsection{Photolithography}

Photolithography utilizes a high-resolution ( $<50 \mathrm{~nm}$ RMS roughness) chrome-on-quartz mask during the exposure method, as shown in Figure 3.4. The mask is placed in close proximity to the layer to be patterned during UV exposure to activate the desired regions of the polymer that will polymerize during a post bake. Since the WG material acts like a negative photoresist the final polymer pattern will resemble the transparent design in the photolithographic mask. 


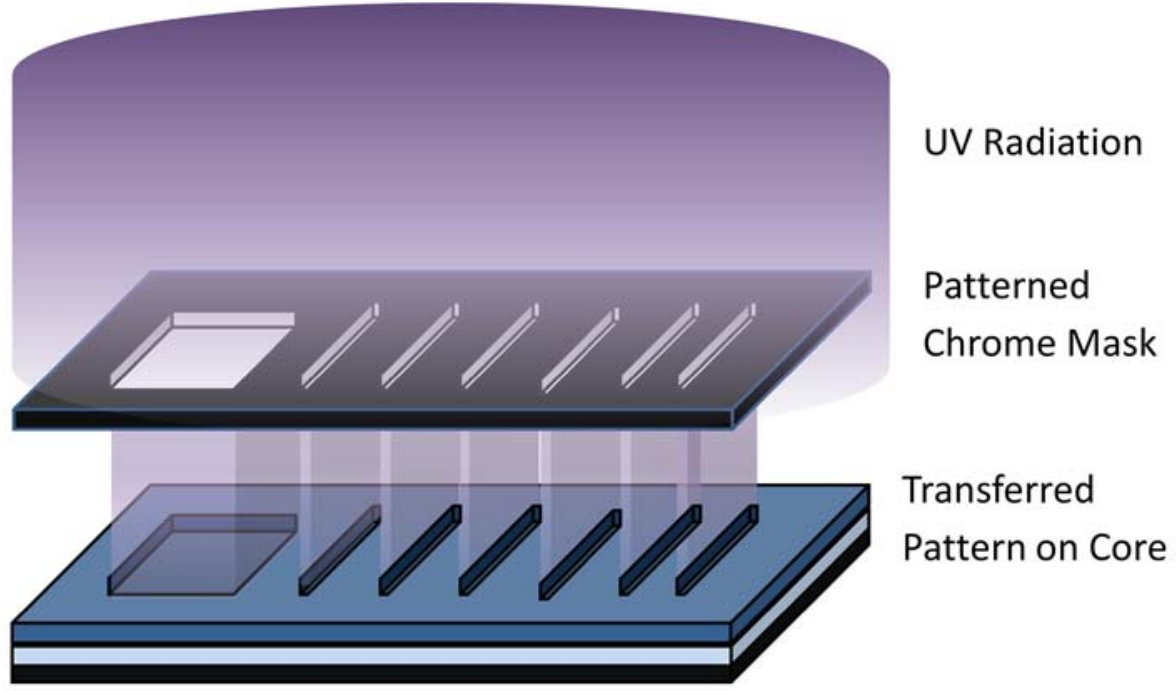

Figure 3.4: Illustration of the photolithographic process.

Photolithography is the traditional method used in fabricating polymer WGs using UVcurable materials for multiple reasons. Exposure times are well under two minutes making it a fast and efficient process with high-throughput. It is also a PCB and CMOS compatible technology, requiring minimal unaccustomed procedures for prospective manufacturers. While promising, the main limiting factors for photolithography are mask cost and resolution limited by diffraction.

While most WG fabrication for research and development is performed on substrates under six inches PCB integration will desire WG builds on large panels (12" x 18”). WG masks require high resolution for minimal sidewall roughness and the fabrication costs increases exponentially with increasing mask size. While one mask can imprint thousands 
of builds, the mask's design is static. While ideal for mass production of a single design manufacturing small quantities of large scale builds quickly becomes cost-limited.

Diffraction also plays a limiting factor in the minimum resolution feasible with photolithography. Dow Corning's material is fairly adhesive and direct contact between the mask and the spun core layer damages the fabrication sample. Adhesion also contaminates the mask incurring wear from material adhesion and required mask cleanings. To alleviate these issues a narrow proximity gap [10 - $100 \mu \mathrm{m}]$ should be placed between the mask and the sample. Furthermore, the core layer is thick [6 - $50 \mu \mathrm{m}$ ] in comparison to most spin-on photoresists. To ensure effective pattern transfer the transmitted UV energy must be able to propagate through the proximity gap and the material layer while maintaining its geometrical dimensions. Unfortunately, the UV pattern will exhibit diffraction effects that increase with longer propagation distances (gap separation and material thickness) and smaller feature sizes. Smaller features within the mask will significantly broaden the optical pattern transferred resulting in a wider structures dosed at lower intensity levels, as shown in Figure 3.5. If the WG dimensions required are smaller than what is feasible with UV-curing methods non-traditional fabrication methods including E-beam lithography [61] have to be pursued. 

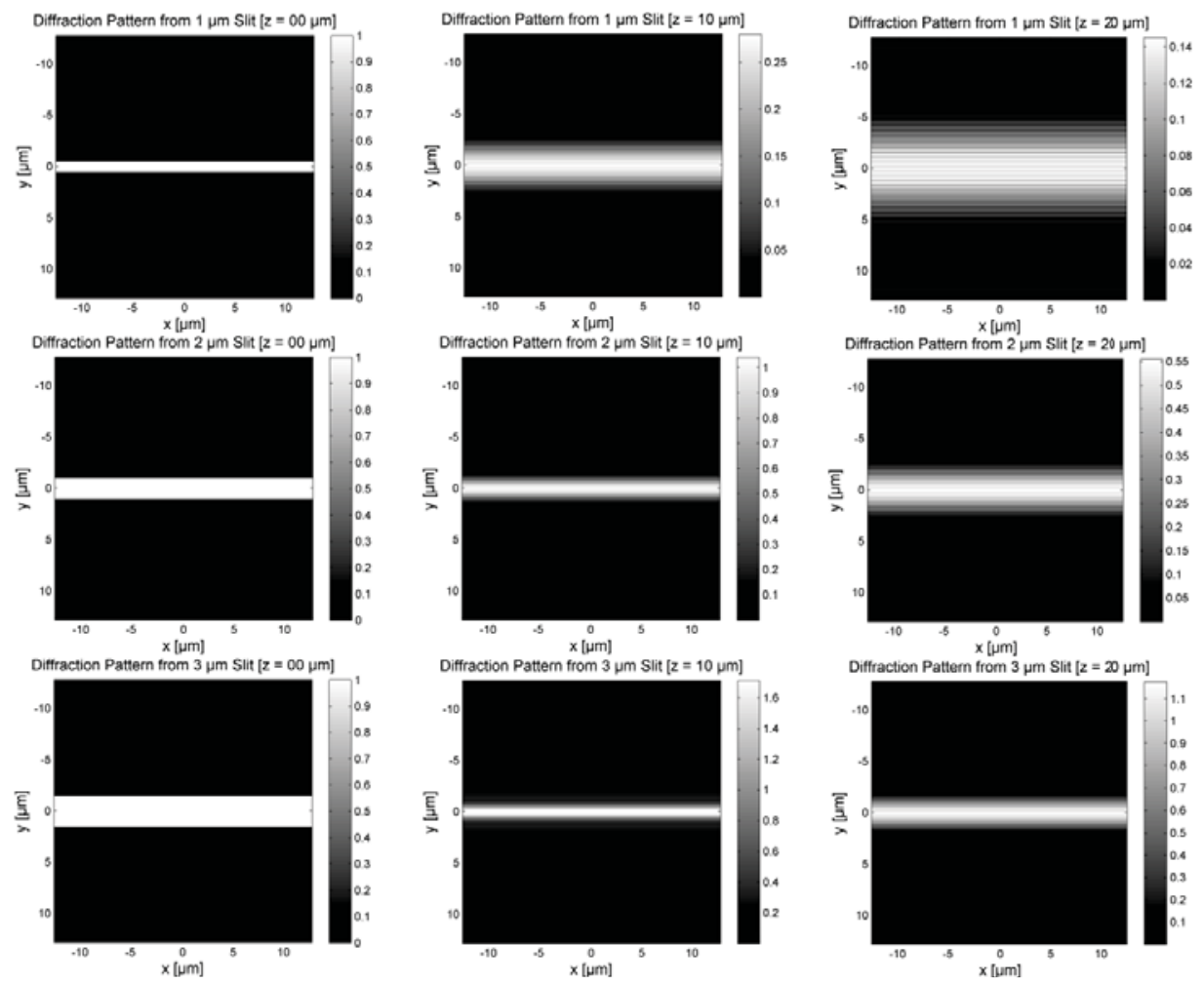

Figure 3.5: Calculated diffraction patterns at various propagation distances from mask slit of various widths.

\subsection{Testing and Evaluation}

Optical interconnect prototyping requires device testing procedures to evaluate WG functionality and improve the manufacturing efficiency and consistency. Testing is done on many levels, including bulk optical material testing, optical WG loss testing, and signal transmission testing. The various techniques to measure the optical properties of optical WG devices are briefly discussed. 


\subsubsection{Refractive Index Testing}

The RI of polymer WG material needs to be accurately known to determine minimum bend radii and WG dimensions for SM functionality. The simplest form of RI measurement is the prism coupling method [62]. With the spin-coated polymer material firmly pressed against a standard prism, as shown in Figure 3.6, the material's optical properties can be measured from a plot of reflection intensity from a laser source as a function of incidence angle. For bulk material a drop in the reflected power at the prism/polymer boundary gives the critical angle for total internal reflection, and the polymer's RI can be calculated according to Snell's law. If the layer is thin enough, specific incidence angles will couple light into slab modes within the sample allowing for accurate measurements in the material's RI and spun thickness based on mode coupling theory [63].

The prism coupling method is limited to the evaluation of the material's RI at the laser source's wavelength. A more detailed optical measurement can be obtained through ellipsometry [64]. This method measures the attenuation and phase shift difference between two orthogonal polarizations reflected off a thin film sample. The acquired data utilizes a model to indirectly determine the physical, chemical, and optical properties of the polymer layer. This is both ellipsometry's strongest and weakest standpoints. Utilizing a filtered broadband source ellipsometric data is correlated to the Cauchy equation to obtain a RI plot of the material as a function of wavelength. However, special care needs 
to be taken in designing the model for unknown polymer compositions to obtain accurate results.

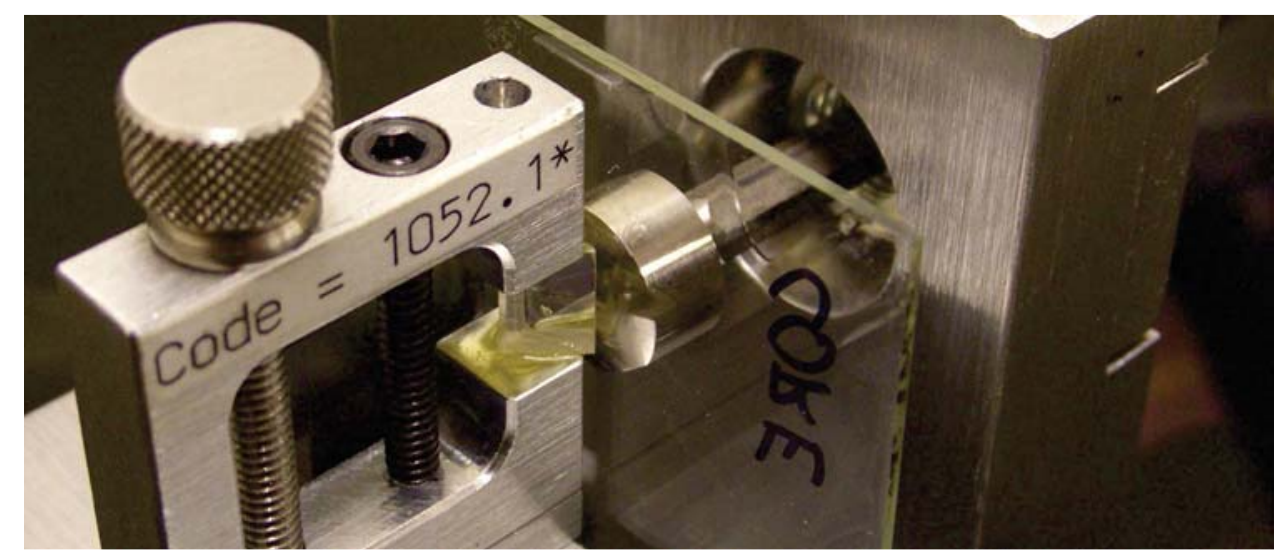

Figure 3.6: Prism coupling method conducted on core material spin-coated on a glass substrate.

\subsubsection{End-fire Coupling Method}

The end-fire coupling method is an efficient way to measure the optical loss of dielectric WGs. Optical power is coupled into fabricated polymer WGs by aligning laser diodecoupled SMF with a high-precision XYZ alignment stage. To isolate the signal from environmental optical noise, the transmitted power is collected with a second SMF and measured using a fiber power detector. The end-fire coupling method setup is shown in Figure 3.7. SM WGs were optically tested using the output of a SM fiber $(\mathrm{NA}=0.14)$ pigtailed laser diode with an operating wavelength of $1310 \mathrm{~nm}$. The launch condition for MM WGs was the output a $2 \mathrm{~m}$ long section of loosely wound $50 \mu \mathrm{m}$ step-index MM 
fiber $(\mathrm{NA}=0.2)$ coupled with a SM fiber $(\mathrm{NA}=0.14)$ pigtailed laser diode operating at a wavelength of $830 \mathrm{~nm}$.

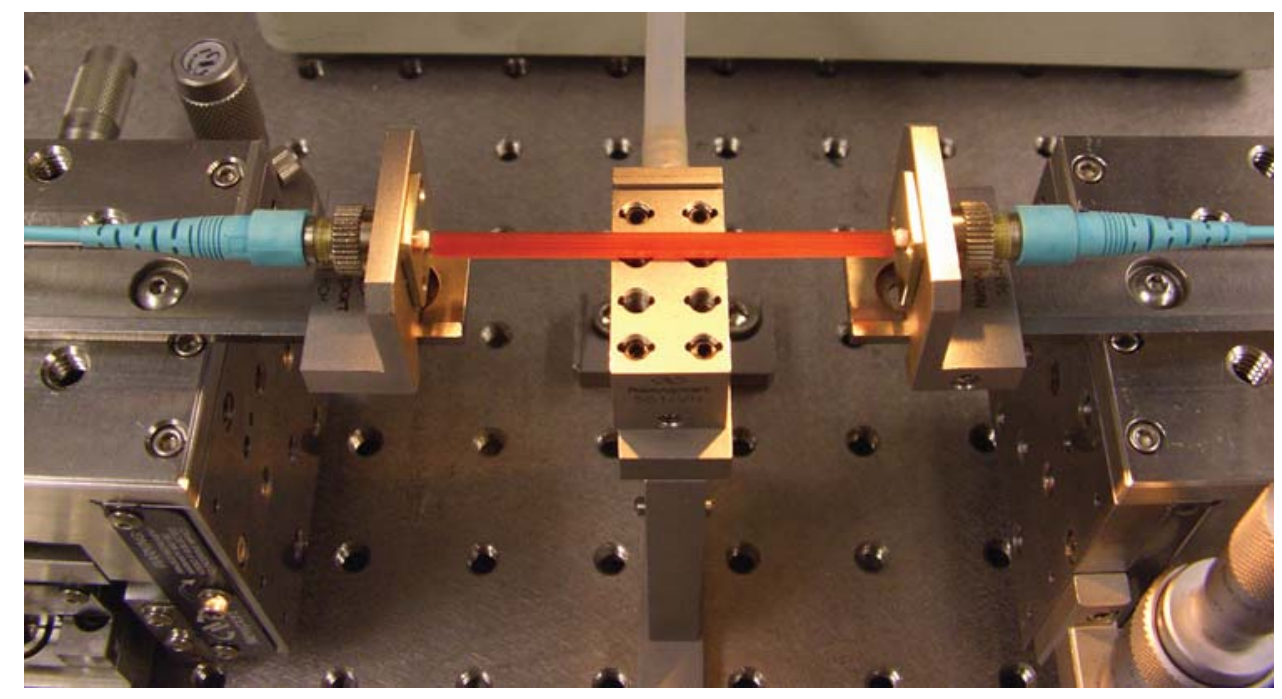

Figure 3.7: The End-fire Coupling Method.

Proper sample preparation procedures are required to minimize coupling loss between WG-SMF coupling [65]. WG samples were milled at the end-facets with multiple drill bits of descending coarseness to minimize WG end-face roughness reducing coupling loss [66]. Index-matching fluid is applied to minimize scattering and reflection loss at the SMF-WG interfaces. A cross-section of the milled end-face of a SM polymer WG is shown in Figure 3.8. 


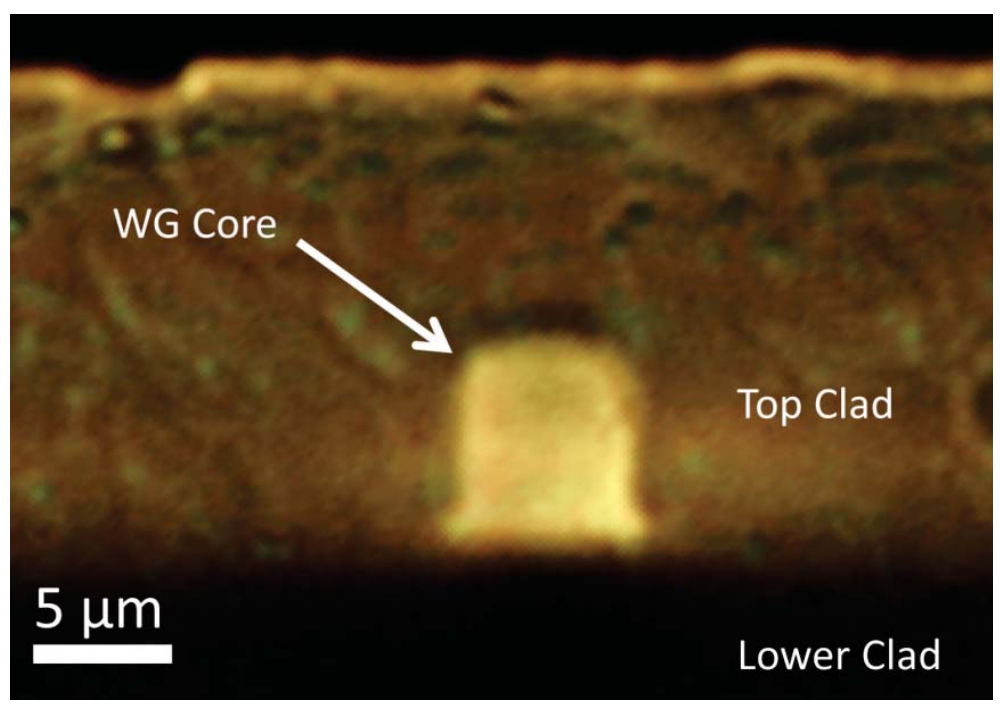

Figure 3.8 Cross-section image of a $6 \times 6 \mu \mathrm{m}$ SM polymer $W G$.

\subsubsection{Cut-back Method}

When WGs are physically measured with the end-fire coupling method additional losses are incurred from coupling losses between the SM WGs and the SMF due to size and NA mismatch. Since coupling losses are independent of WG length, propagation loss can be isolated by plotting optical device loss as a function of WG length though the cut-back method [67]. Unfortunately, the cut-back method is a destructive testing method, permanently altering and destroying the WG sample. The cut-back method is conducted by consistently dicing back a long polymer WG sample to shorter distances. In between individual dicings the WG sample is optically measured at specific cut length. By plotting WG loss vs. WG length, as shown in Figure 3.9, propagation losses are observed as being 
length dependent $(\mathrm{dB} / \mathrm{cm})$ with the y-intercept showing WG/SMF coupling loss for both end-faces.

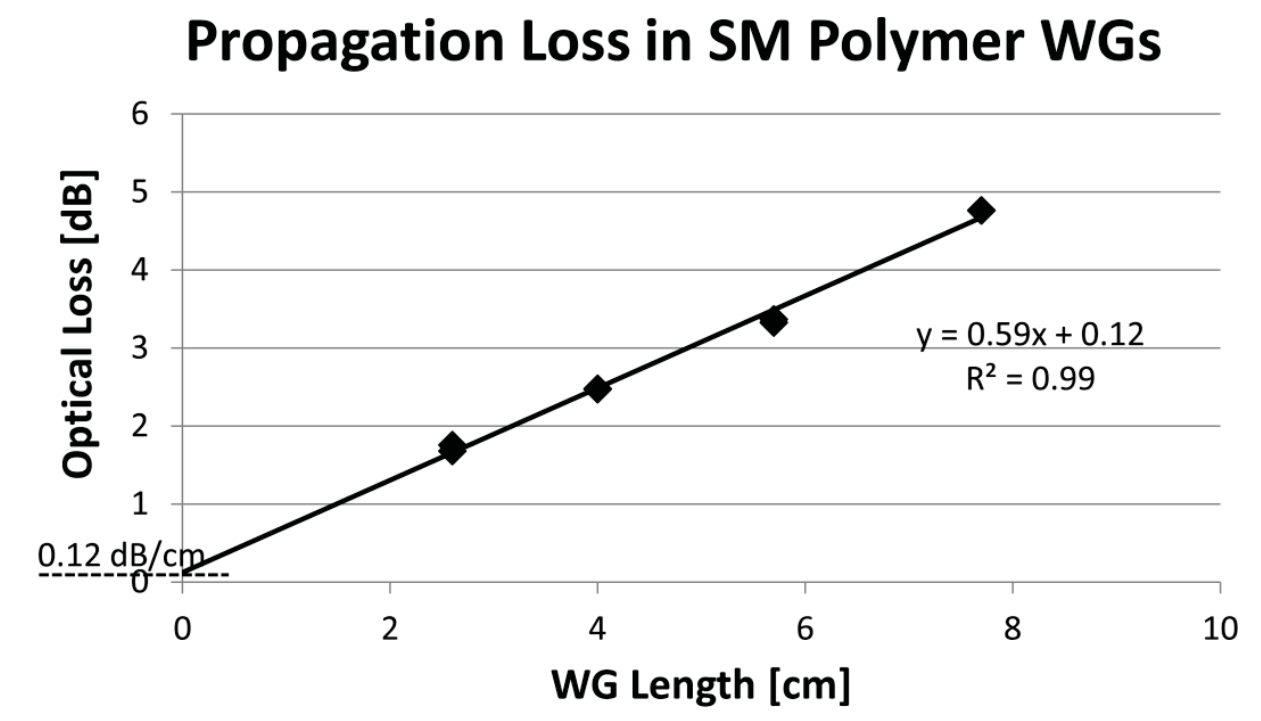

Figure 3.9 Loss plot for photolithographic SM polymer WGs from the cutback method.

Propagation loss of $0.59 \mathrm{~dB} / \mathrm{cm}$ were measured from conducting the cut back method for SM polymer WGs. A low coupling loss of $0.06 \mathrm{~dB} /$ facet was also observed due the similar NA values between the SM WGs $(\mathrm{NA}=0.135)$ and SMFs $(\mathrm{NA}=0.14)$. Measuring optical output power of $6 \mu \mathrm{m}$ SM WGs in relation to SMF displacement, as shown in Figure 3.10, the SM WG's $1 \mathrm{~dB}$ and $3 \mathrm{~dB}$ alignment tolerances were $\pm 2.0 \mu \mathrm{m}$ and $\pm 3.5 \mu \mathrm{m}$ respectively. 


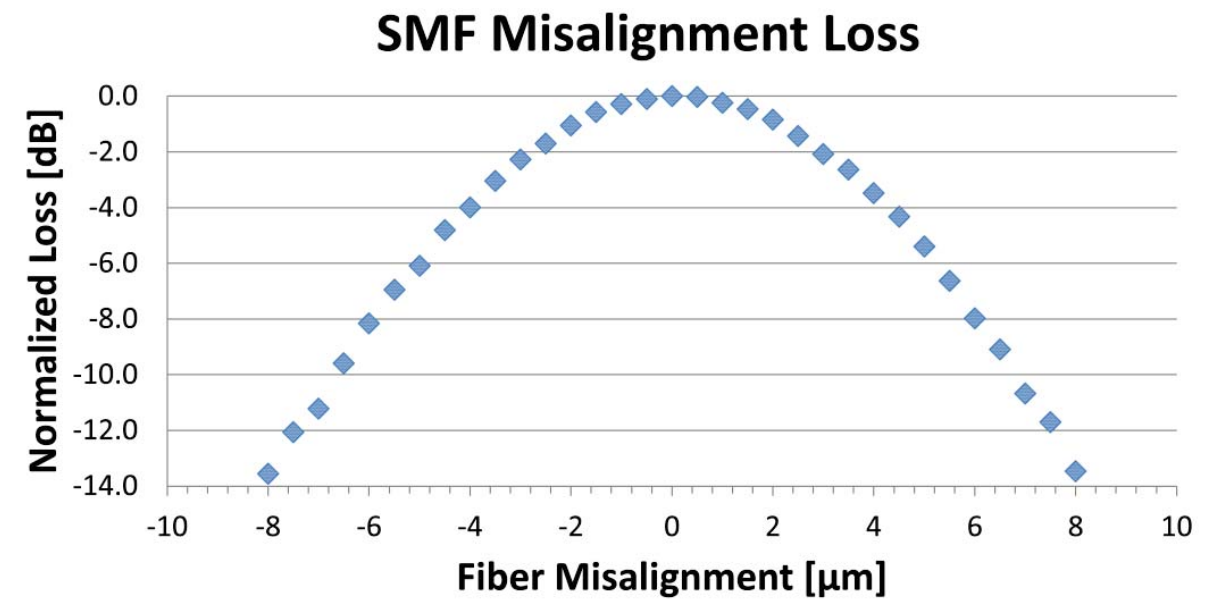

Figure 3.10 Normalized measured misalignment loss between a SMF and SM polymer $W G(\lambda=1310 \mathrm{~nm})$.

\subsubsection{Signal Quality Analysis}

Signal quality analysis is utilized to demonstrate the WG's ability to transmit optical data at high data rates. Signal quality analyzers output high-power, high-quality electrical signals that drive optical signals through commercially-available optical transceivers. After propagating through the WG sample the transmitted optical signal is recollected, converted back to an electrical signal, and compared with the input signal. Signal comparison and error detection modules allow for the calculation of signal-to-noise ratios (SNR), bit error rates, and signal jitter, and other effects from signal dispersion and distortion.

Signal quality can be visually observed with an eye diagram analyzer. Eye diagram analyzers display and measure signal ramp-rates, noise, synchronization, clearance, and 
overlap. While not as accurate as high-end signal quality analyzers, eye diagrams allow for signal visuals to assist in the troubleshooting of unstable and dysfunctional transmission lines. Bit error rate testing at $10 \mathrm{GBits} / \mathrm{sec}$ was conducted on $3 \mathrm{~cm}$ long samples of SM WG samples since effective data transmission in SM WGs is important for their realization as high-bandwidth optical interconnects. As shown in Figure 3.11, SM WG straights and bends show open eye diagrams and effective high-speed data transmission.
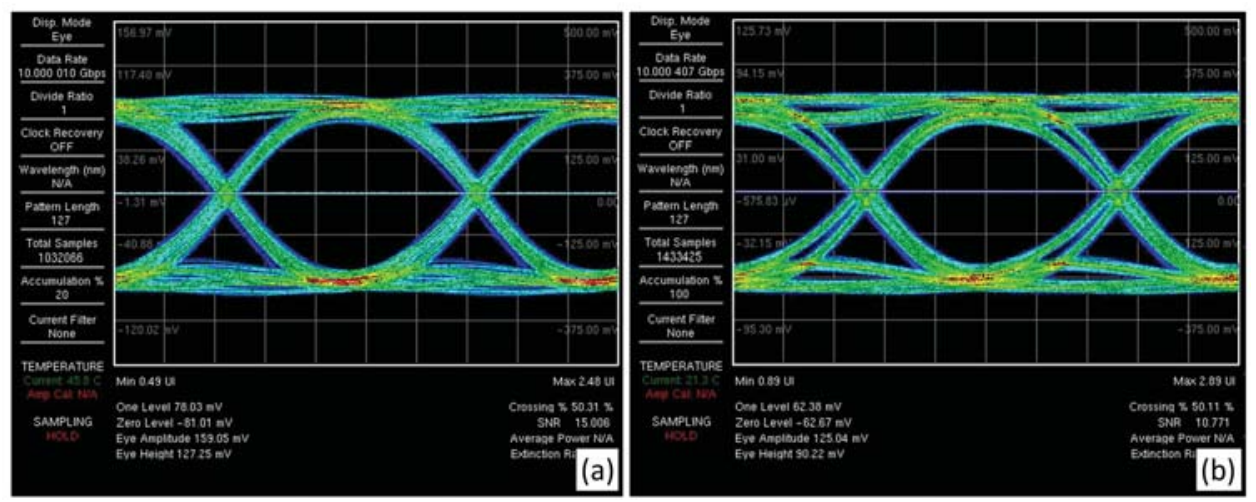

Figure 3.11 Eye diagrams at 10 GBits/sec for SM WG straights (a) and SM WG bends (b).

\subsection{Fabrication Summary}

UV-curable optical elastomers available from Dow Corning ${ }^{\circledR}$ were fabricated into SM polymer WGs through the photolithographic method. SM polymer WGs measured using the cut-back method exhibited propagation loss $(0.6 \mathrm{~dB} / \mathrm{cm})$ with high correlation to theoretical absorption $(0.4 \mathrm{~dB} / \mathrm{cm})$ loss demonstrating low levels of optical scattering from sidewall roughness. Low (0.06 dB/end-face) loss optical coupling was observed 
between SM polymer WGs and SMFs with $\pm 3.5 \mu \mathrm{m}$ alignment $(<3 \mathrm{~dB})$ tolerances. High (10 Gbits/sec) data transmission was evaluated through eye diagram analysis to show high quality optical communication. 


\section{Chapter 4: Laser Direct Write Method}

While photolithography is optimal for high-throughput manufacturing it is an extremely expensive investment that risks development funds while scaling up optical interconnect commercialization. In this Chapter the laser direct writing (LDW) method is introduced and implemented as an alternative cost efficient polymer WG fabrication method for prototyping large substrates for optical backplanes and optical interconnection assemblies. The LDW platform is a mask-less procedure that utilizes a 2-axis air-bearing motion platform equipped with a UV laser diode coupled single mode fiber with a heightadjustable focusing lens module. The LDW platform is capable of LDW WGs for both MM WG $(50 \mu \mathrm{m})$ and SM WG $(6 \mu \mathrm{m})$ dimensions. Correlation between LDW parameters and fabricated WG dimensions is discussed theoretically and confirmed experimentally for both MM and SM applications. LDW MM and SM WGs are experimentally produced showing comparable losses photolithographic WG builds. LDW

1. Research in this chapter has been previously published by the author in the following reference:

- K. Kruse and C. Middlebrook, "Laser-direct writing of single mode and multi-mode polymer step index waveguide structures for optical backplanes and interconnection assemblies," Photonics and Nanostructures-Fundamentals and Applications, 2014. 
MM and SM WG radial bends and crossing losses are evaluated for advanced optical communication channel routing capabilities and do not exhibit significant deviations from photolithographic-manufactured WG device loss.

\subsection{Introduction}

Due to the exponentially increasing costs of high-resolution masks photolithography becomes increasingly difficult and expensive for the prototyping of large (>12") scale builds as discussed in Chapter 3. An alternative fabrication method for WG prototyping is the laser direct writing (LDW) method [21, 22]. LDW allows for the flexible, mask-less fabrication of WGs through the use of a UV writing beam mounted on an XYZ movement stage. The focused UV beam is then scanned along the core material to selectively initiate polymerization within the applied core layer, illustrated in Figure 4.1. The LDW process presents a non-contact fabrication method of curing UV optical polymers at writing heights greater than $1 \mathrm{~mm}$.

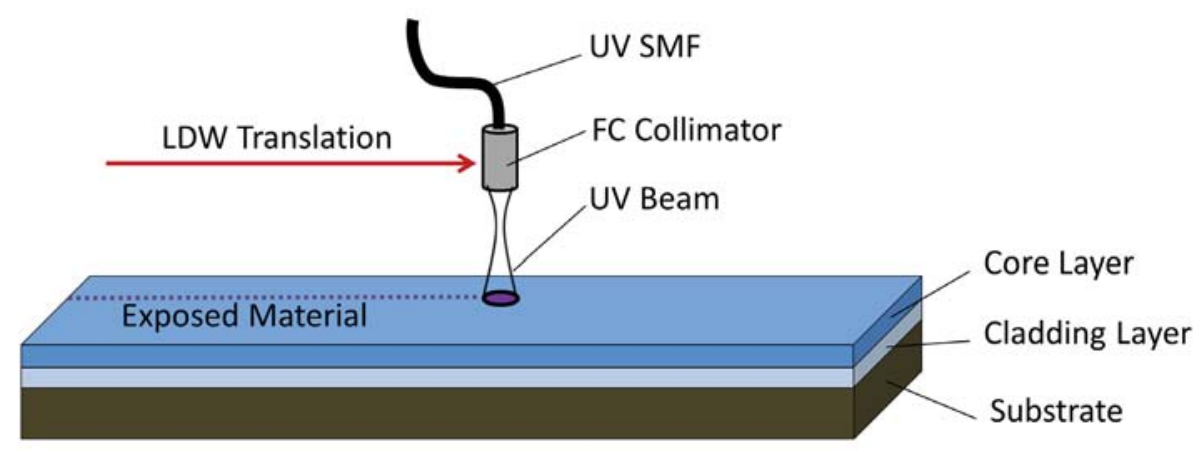

Figure 4.1: Theoretical setup of the LDW process. 
The main drawback of the LDW process is manufacturing speed. Individually writing each WG drastically increases the required fabrication time per sample. While photolithography patterning is done in $<2$ minutes similar builds fabricated under LDW will take over an hour ( $\sim 100 \mathrm{~m} / 300$ polymer WGs at $30 \mathrm{~mm} / \mathrm{s})$ for complete UV initiation. Nonetheless, the LDW WG patterns and layouts can easily be altered though motion programming of the linear stages used within the platform. This process exhibits excellent accuracy, flexibility, and scalability without incurring the high of costs of highresolution photolithographic masks, making the LDW method cost-efficient and appealing for WG prototyping [60]. LDW is an excellent fabrication method for WG build prototyping and small-batch manufacturing.

Previous LDW theoretical curing profile models $[22,23]$ are not compatible with the Dow Corning $^{\circledR}$ optical elastomers due to their unique proprietary chemical properties and curing kinetics. A new theoretical prediction model was developed to effectively correlate writing parameters with WG dimensions. The theoretical model incorporates Dow Corning Elastomers and similarly-reactive materials for effectively performing LDW. The high-precision motion platform with an optimized UV source paired with a high-sensitivity optical elastomer makes this process to the best of the author's knowledge the fastest, most accurate LDW procedure for the fabrication of WGs with rectangular cross-section profiles [22, 38, 68, 69]. 


\subsection{Theoretical Model}

Waveguide dimensions fabricated through the LDW process are dependent on the spatial and temporal properties of the focused UV beam. The irradiance profile of the UV writing beam can be represented as a symmetric Gaussian beam [37] given by Eq. (4.1). $P_{t}$ is the total output power and $W(z)$ is the width of the Gaussian writing beam that is dependent on the writing height, $z$.

$$
I(\rho, z)=\frac{2 P_{t}}{\pi W^{2}(z)} \exp \left[-\frac{2 \rho^{2}}{W^{2}(z)}\right], \quad \rho=\sqrt{x^{2}+y^{2}}
$$

The material will experience an intensity curing profile during the LDW process consistent with a Gaussian curve along the axis normal to the direction of propagation. This intensity profile is obtained by taking the marginal probability distribution function of Eq. (4.1). The result is shown in Eq. (4.2). Multiplying Eq. (4.2) by the dwell time, $t_{d}$, results in Eq. (4.3) the final energy curing profile of the LDW process. The dwell time is proportional to the diameter of the curing profile divided by the writing speed.

$$
\begin{aligned}
& I(x, z)=\frac{\sqrt{2} P_{t}}{\sqrt{\pi} W(z)} \exp \left[-\frac{2 x^{2}}{W^{2}(z)}\right] \\
& E(x, z)=\frac{\sqrt{2} P_{t} t_{d}}{\sqrt{\pi} W(z)} \exp \left[-\frac{2 x^{2}}{W^{2}(z)}\right]
\end{aligned}
$$

The WG polymer requires a minimum level of UV energy to initiate polymerization before thermal curing to solidify the material, making it resistant to solvent washing. 
Increasing the amount of UV exposure present during the writing process increases the region in the Gaussian curing profile that overcomes the energy threshold. This results in larger regions of polymer that will undergo solidification and form wider WGs. This effect is represented in Figure 4.2.

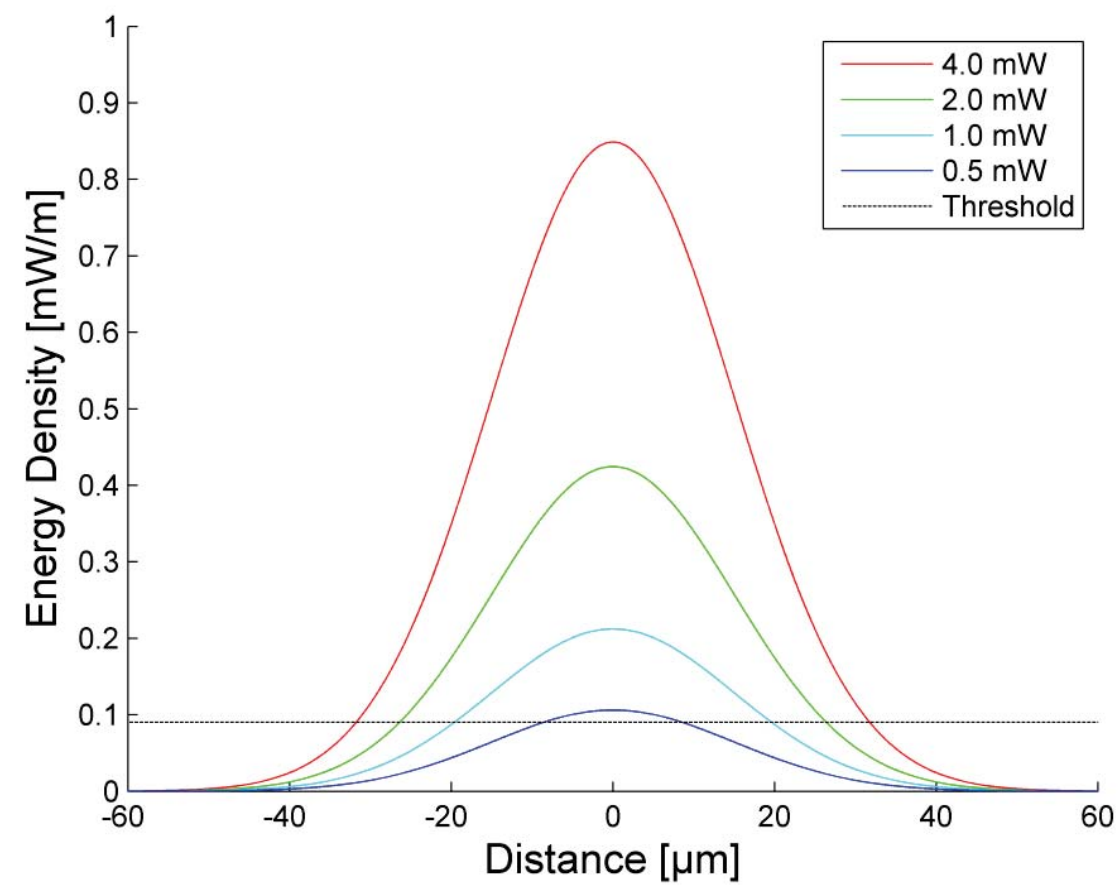

Figure 4.2: Gaussian curing model at various power levels in relation to the energy threshold required for polymerization.

By manipulating Eq. (4.3) and setting $E(x, z)=E_{\text {thresh }}$, the threshold energy required to initiate solidification, we can show that the width of the WG increases logarithmically in respect to higher total output power and longer dwell time. This correlation is given by Eq. (4.4). 


$$
W G \text { Width }=2 W(z) \sqrt{-\frac{1}{2} \ln \left(\frac{E_{\text {thresh }} \sqrt{\pi} W(z)}{\sqrt{2} P_{t} t_{\text {dwell }}}\right)}
$$

\subsection{Experimental Setup Procedure}

A commercially available UV diode laser $(\lambda=375 \mathrm{~nm})$ was used as the UV writing source. The Dow Corning ${ }^{\circledR}$ UV-Cured Optical Elastomer exhibits high absorbance at this wavelength, as shown in Figure 4.3, which maximize the polymer's UV sensitivity for the LDW process when utilizing high-power UV laser diodes $(\lambda>350 \mathrm{~nm})$ [13].

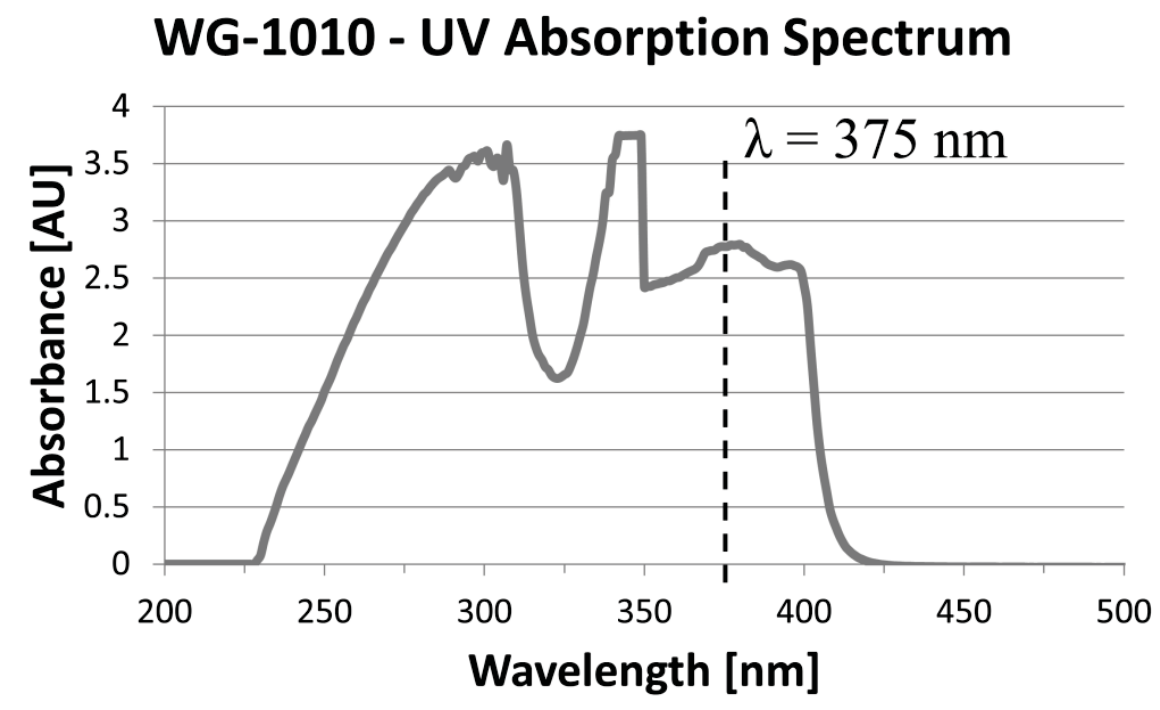

Figure 4.3 UV Absorption Spectrum of the Dow Corning WG Material.

The Toptica ${ }^{\odot}$ iBeam Smart, a 375 nm UV Laser module, was fiber coupled to a UV single-mode fiber (SMF) with the Toptica ${ }^{\circledR}$ SmartDock fiber coupler, which exhibits a laser-to-SMF coupling efficiency of $55 \%$. The output power was coupled into a UV 
single-mode fiber for two reasons; to allow for flexibility in maneuvering the UV power and isolating the laser module from the LDW system. The UV SMF also spatially filters the optical profile. The propagating fundamental mode exits as a smooth Gaussian profile. This circularly symmetric beam allows for direction-independent writing parameters and reduces any unwanted sidewall roughness in the fabricated LDW WGs.

A 2-mm focal length aspheric lens was introduced after the SMF output to form a convergent UV beam. The LDW output exhibits a circularly symmetric beam irradiance profile. The resulting smooth Gaussian profile, shown in Figure 4.4, allows for directionindependent writing parameters and limits the sidewall roughness in the fabricated LDW WGs. The width of the output Gaussian profile can be controlled by altering the writing height, $z$, above from the substrate. The output profile was measured with a scanning slit beam profiler [DataRay, Inc.]. The lensed SMF output matches a Gaussian profile with a Gaussian fit of $>90 \%$ and a beam ellipticity of $1.01 \pm 0.05$. The focus of the convergent beam was measured to have a FWHM and $1 / \mathrm{e}^{2}$ diameter of $4 \mu \mathrm{m}$ and $8 \mu \mathrm{m}$ respectively, as shown in Figure 4.5. The beam quality factor, $M^{2}$, was calculated at $M^{2}=1.33$ from measured UV beam widths as a function of normalized writing height shown in Figure 4.6.

The SMF output and focusing lens were rigidly mounted on an XYZ motion platform equipped with air-bearing linear stages [Aerotech, Inc.]. The platform's measured position and velocity root mean squared (RMS) errors during LDW were $\pm 0.15 \mu \mathrm{m}$ RMS 
and $\pm 4.14 \mu \mathrm{m} / \mathrm{s}$ RMS respectively after adjusting the platform's gain parameters to optimal writing controls. The platform is shown in Figure 4.7.

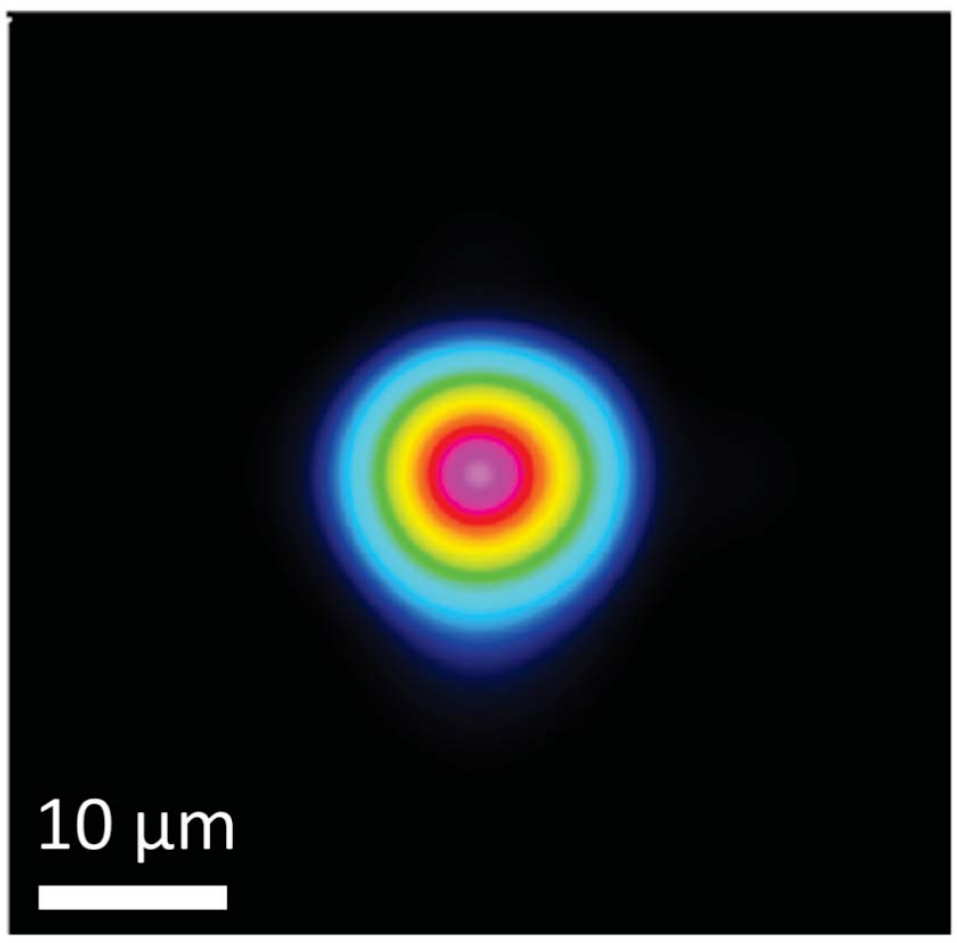

Figure 4.4 3D Beam profile of focused UV SMF laser output. 


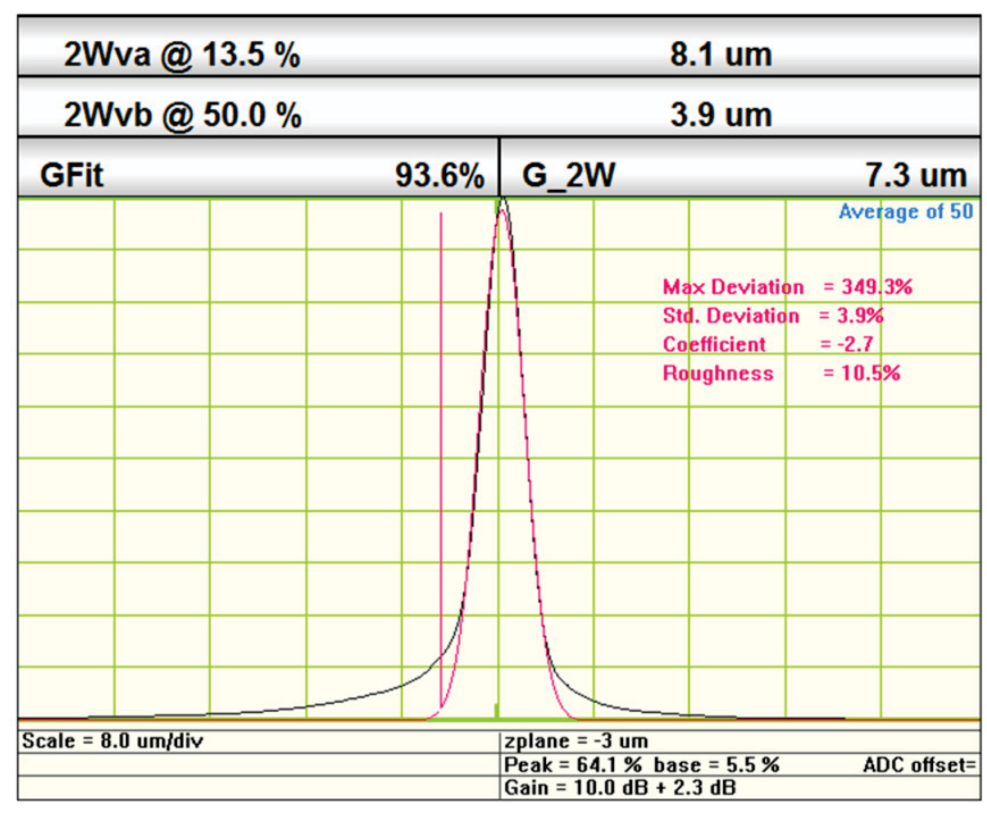

Figure 4.5 2D matched Gaussian fit of focused UV SMF laser output.

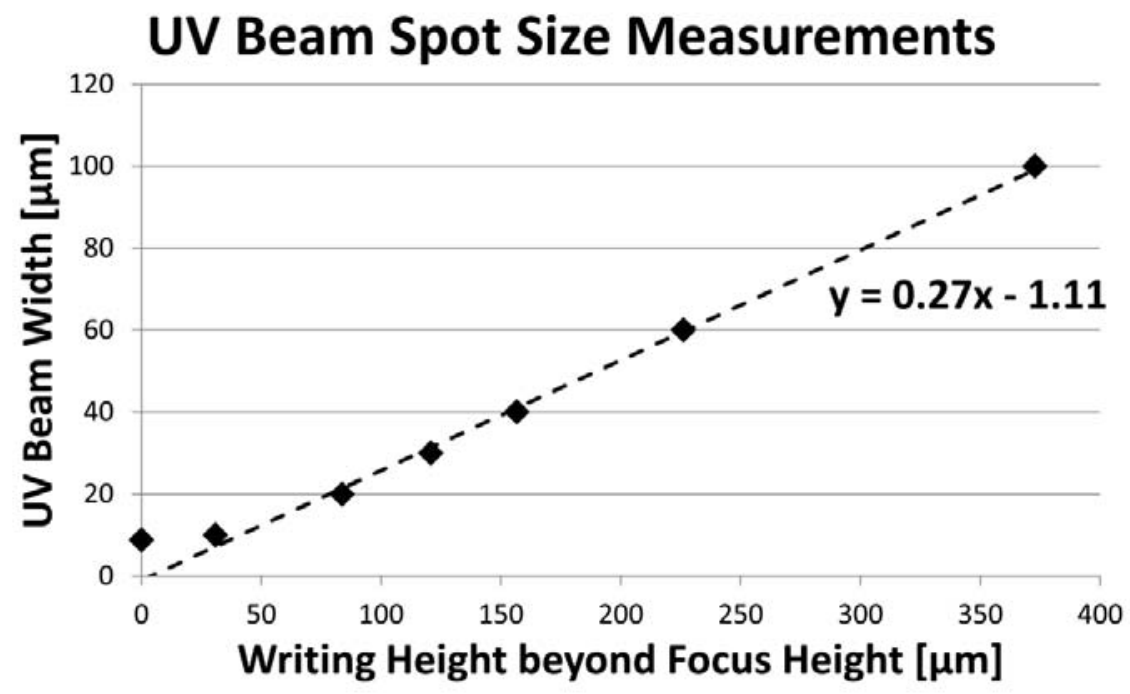

Figure 4.6 Beam divergence observed with increasing $1 / e^{2}$ beam diameter width as the writing height is increased away from focus $(x=0 \mu \mathrm{m})$. 
Conventional programming of numerical controlled (NC) machine tools has been based on a data model stipulated by ISO 6983 [70], or otherwise known as G-code. These algorithms allow the Aerotech platform to efficiently and smoothly write WG straights, turns, and other devices while maintaining a constant velocity during the LDW process. WG designs manufactured with the LDW process are restricted with the limited movement operations programmable within G-code: linear (G1), clockwise (G2), and counterclockwise (G3). These functions can be programmed with a specified travel distance $[\mathrm{mm}]$, movement velocity $[\mathrm{mm} / \mathrm{sec}]$, and ramp rate $\left[\mathrm{mm} / \mathrm{sec}^{2}\right]$. The primary function of linear (G1) operations is to fabricate polymer WG straights. Clockwise (G2) and counterclockwise (G3) operations pattern polymer WG radial bends of constant curvature. 


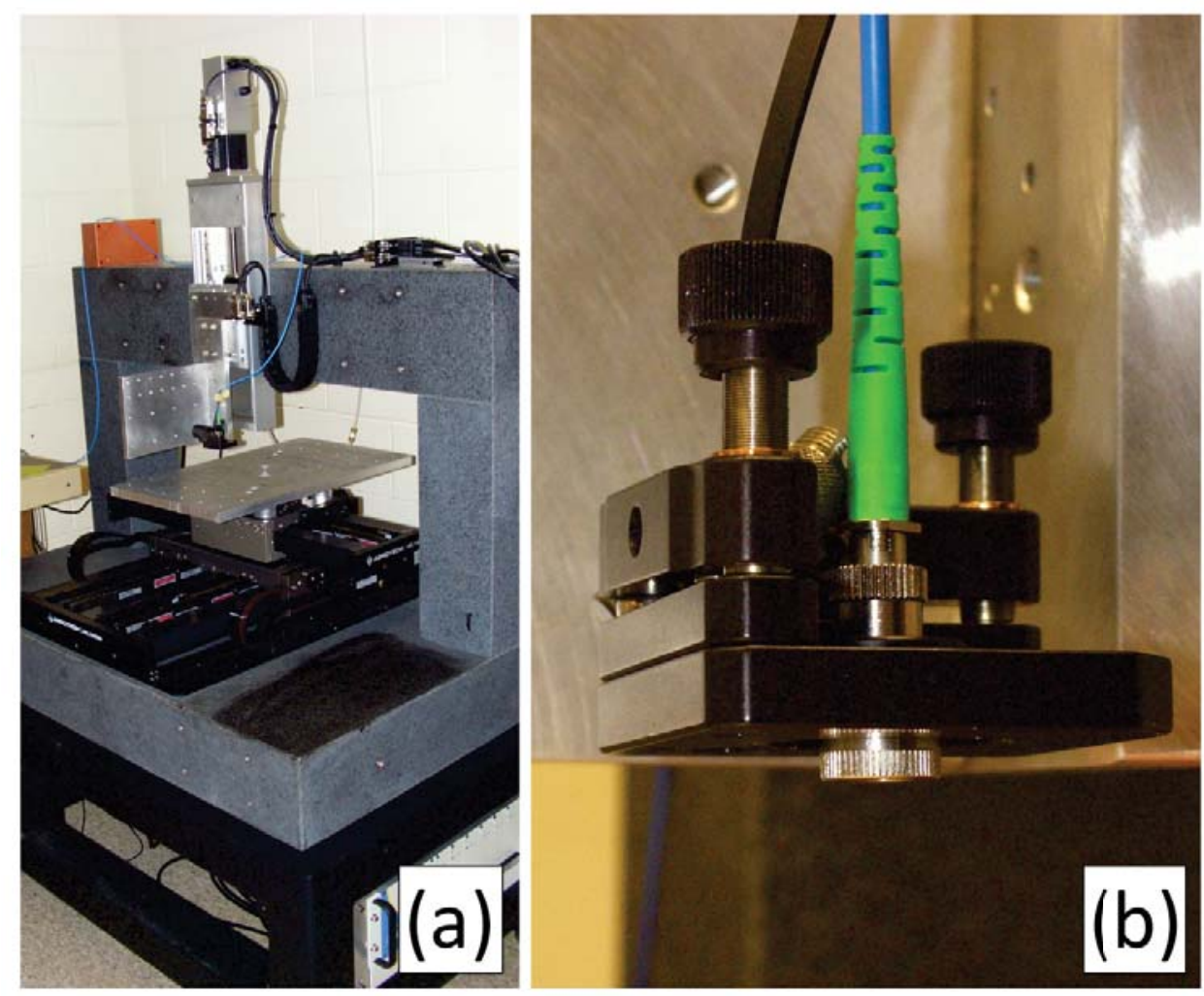

Figure 4.7 The laser direct writing setup consists of an air-bearing XYZ movement stage (a) equipped with a lens-focused UV SMF output (b).

The LDW writing parameters were altered to control the WG widths. Polymer WGs were written at various power levels $[0.5-8.0 \mathrm{~mW}]$ and writing speeds $[10-60 \mathrm{~mm} / \mathrm{s}]$. The writing height, $W(z)$, was altered to adjust the UV writing beam diameter. WG core material was spun down at various thicknesses to reduce WG deformation from highaspect ratio structures. WG core material was spun down to a thickness of $50 \mu \mathrm{m}$ and 6 $\mu \mathrm{m}$ for MM and SM WGs respectively. Symmetric $50 \mu \mathrm{m}$ MM WGs and $6 \mu \mathrm{m}$ SM WGs were obtained with $1 / \mathrm{e}^{2}$ writing beam waists of $60 \mu \mathrm{m}$ and $8 \mu \mathrm{m}$ respectively, as shown in 
Figure 4.8. LDW WG straights, bends, and crossings were fabricated using the previously stated parameters.
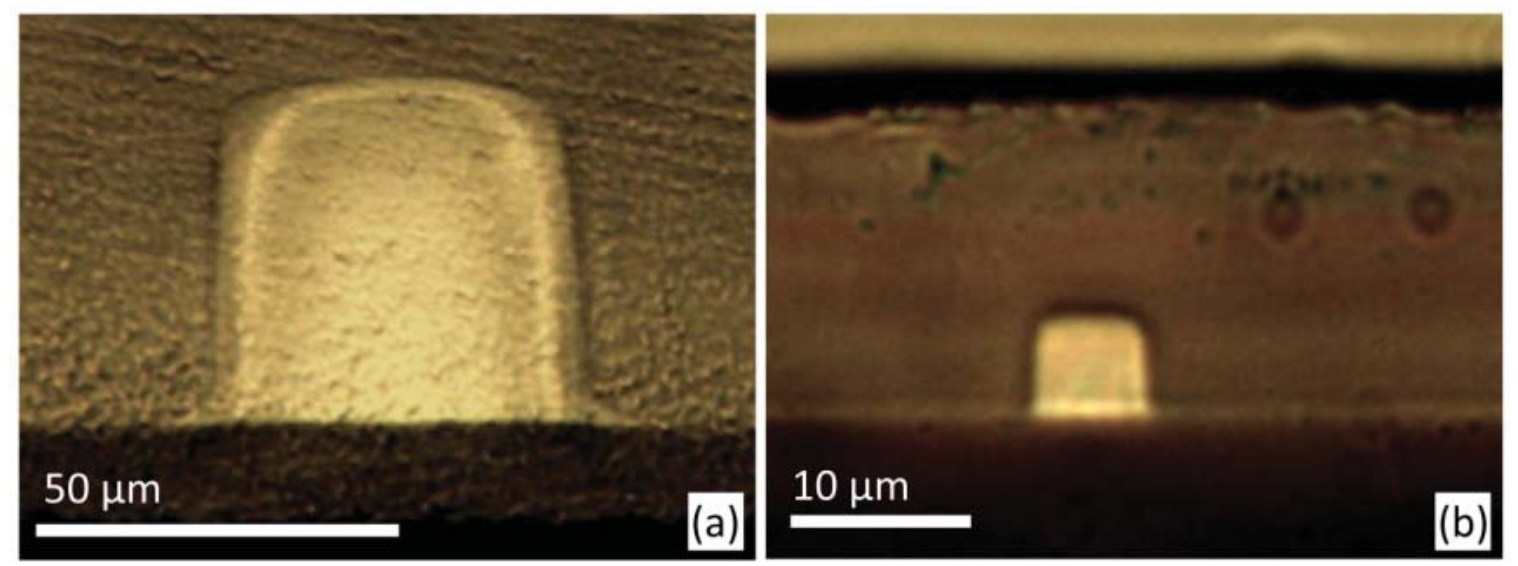

Figure 4.8 Cross-sections of symmetric $50 \mu \mathrm{m} M M($ a) and $6 \mu \mathrm{m}$ SM (b) WGs fabricated through $L D W$.

\subsection{Results and Discussion}

The LDW method was utilized to write polymer WGs with various writing heights, writing speeds, and UV power. WG dimensions of experimental results and correlated to theoretical calculations of WG widths. WG straights, bends, and crossings were also fabricated using the LDW method and their optical losses have been measured to show applicability for manufacturing. These WG devices are applicable for many optical interconnect applications including optical shuffleboards illustrated in Figure 4.9. 


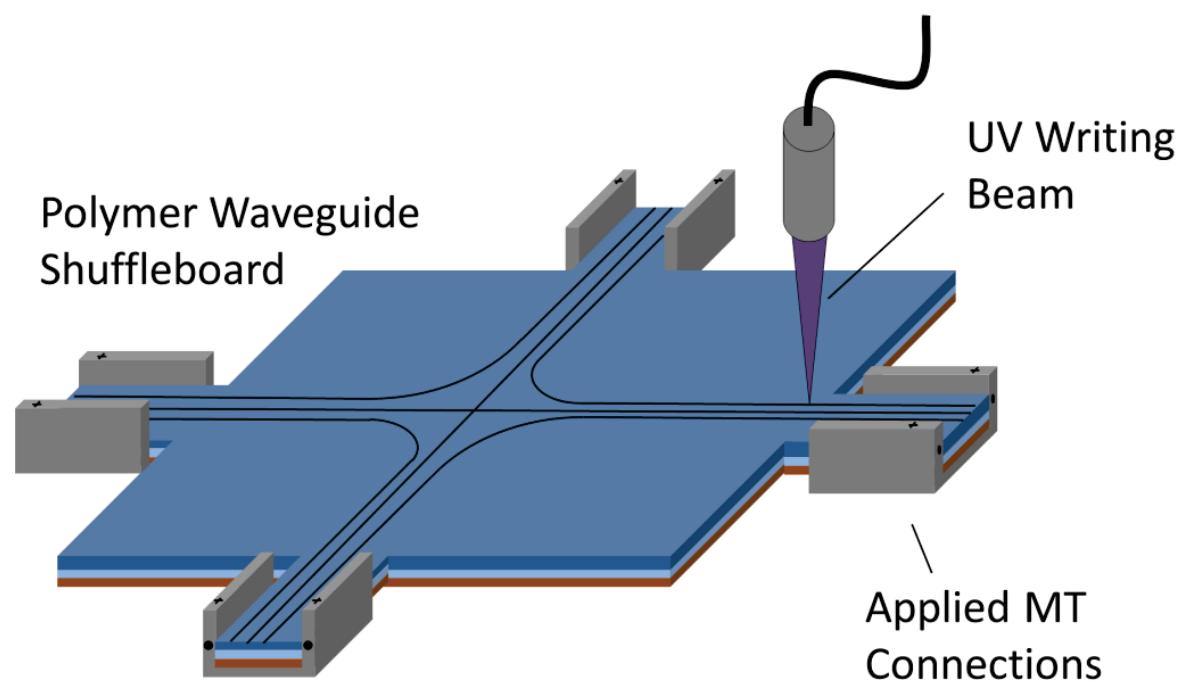

Figure 4.9: WG straights, bends, and crossings are utilized in the design of polymer waveguide shuffleboards.

\subsection{1. $L D W W G$ Dimensions}

Experimental WG widths were plotted and matched with fitted theoretical curves. The thickness of the applied core layer was smaller than the beam profile width to obtain relatively balanced width-to-height aspect ratios. Theoretical beam waist diameters were within $\pm 5 \%$ from measured beam waists. Theoretical energy thresholds, $E_{\text {thresh }}$, of 6.2 $\mathrm{J} / \mathrm{cm}^{2}$ and $3.0 \mathrm{~J} / \mathrm{cm}^{2}$ were obtained to match the experimental results from $60 \mu \mathrm{m}$ and 20 $\mu \mathrm{m}$ beam profiles respectively. Factors that alter the effective energy threshold include material thickness, activator concentrations, substrate reflectivity, and solvent concentration levels. Experimental results and correlated theoretical curves are shown in Figure 4.10 and Figure 4.11. 


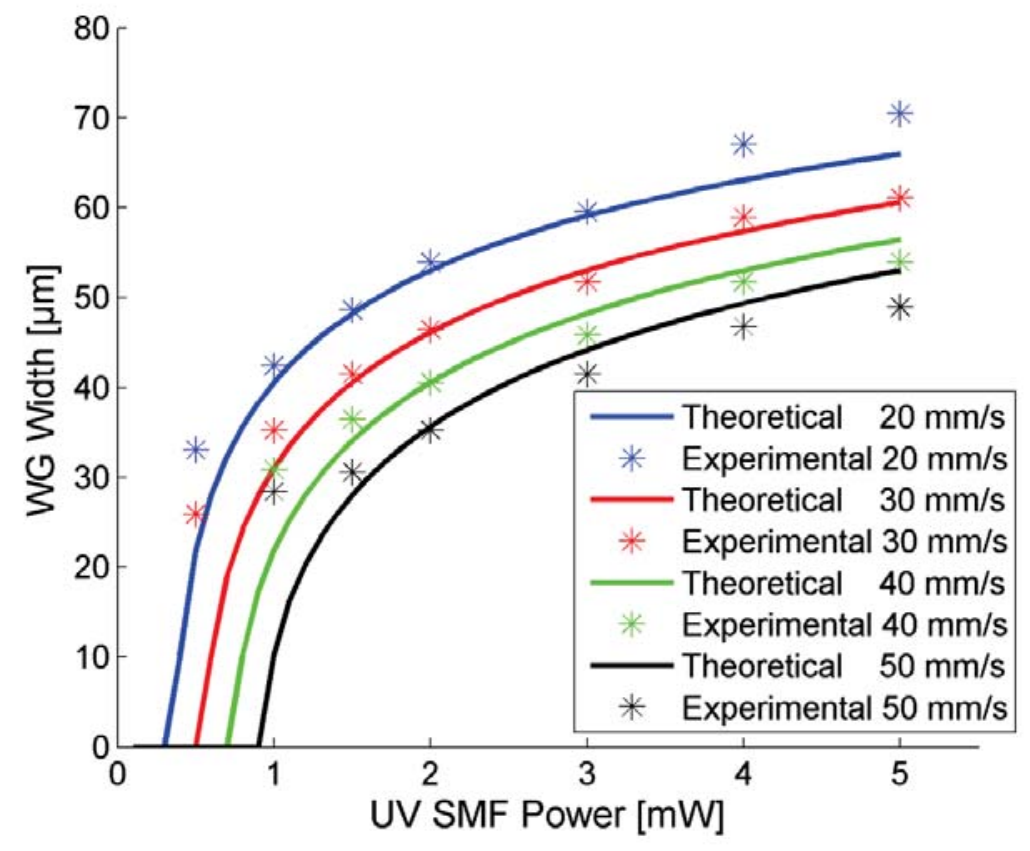

Figure 4.10 LDW WG widths correlated to UV beam diameters of $60 \mu \mathrm{m}$. The polymer core layer thickness applied before LDW was $50 \mu \mathrm{m}$.

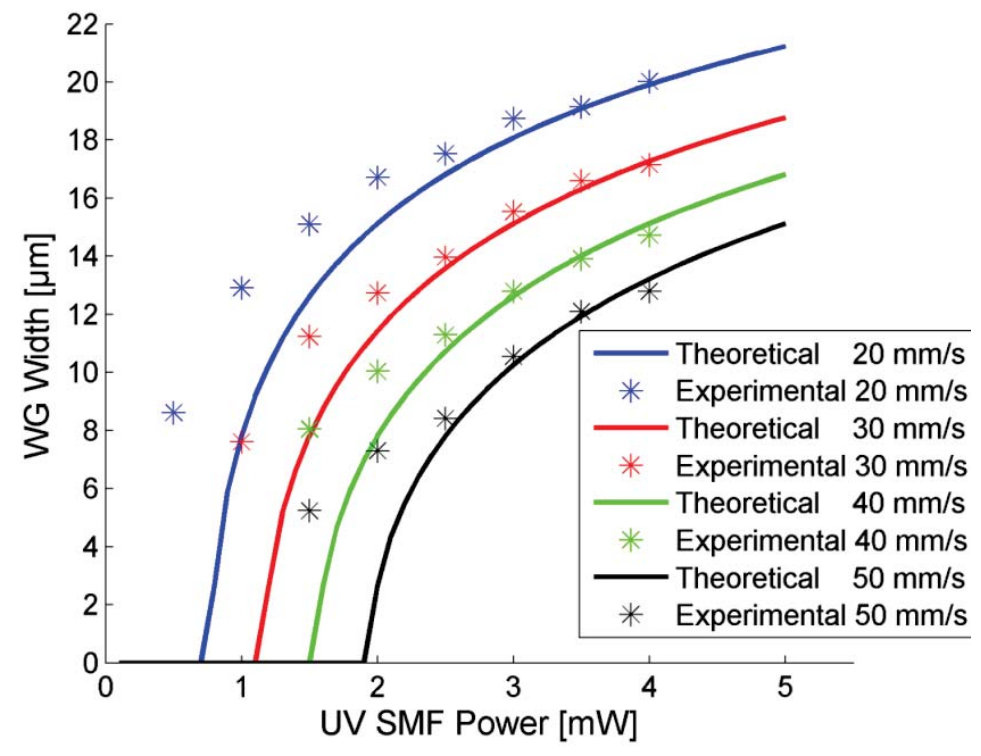

Figure 4.11 LDW WG widths correlated to UV beam diameters of $20 \mu \mathrm{m}$. The polymer core layer thickness applied before $L D W$ and $7 \mu \mathrm{m}$. 
The model also states that the LDW WG's width is only dependent on the total energy dose applied by the UV beam. The WG's height is solely dependent on the applied core layer thickness. LDW MM WGs were fabricated with balanced writing speeds [10-60 $\mathrm{mm} / \mathrm{s}]$ and output powers $[1-7 \mathrm{~mW}]$ to maintain a constant energy dose. Fabricated MM WGs exhibited widths of $54.5 \pm 2.5 \mu \mathrm{m}$ and heights of $50.7 \pm 3.5 \mu \mathrm{m}$. These consistent dimensions show that the LDW process exhibits no non-linearities in writing speed or output intensity.

Similar to photolithography the resolution of the LDW process is limited to the minimal spot size of the UV output. WGs require a beam diameter slightly larger than the target WG width for optimal fabrication, otherwise difficulties arise due to abnormal curing results. There is a significant level of correlation $[< \pm 5 \mu \mathrm{m}]$ between model and experiment when WGs widths are 0.5-1.0x the writing beam diameter. Written WGs in this region also maintained a constant WG height, demonstrating that WG height is purely depended on the thickness of the applied core layer. However, the model fails at both energy extremes, where WG over-curing and under-curing occurs. Visuals of various WGs written at sub-optimal writing parameters are shown in Figure 4.12.

Without enough UV initiation in the polymer, under-exposure resolves in material drooping and collapsing creating WGs that are wider and flatter than expected, shorter in height in comparison to the thickness of the spun core layer. Polymer WGs written at significantly lower energy levels are washed away during the development step as a result of insufficient solidification and adhesion to the lower cladding. 

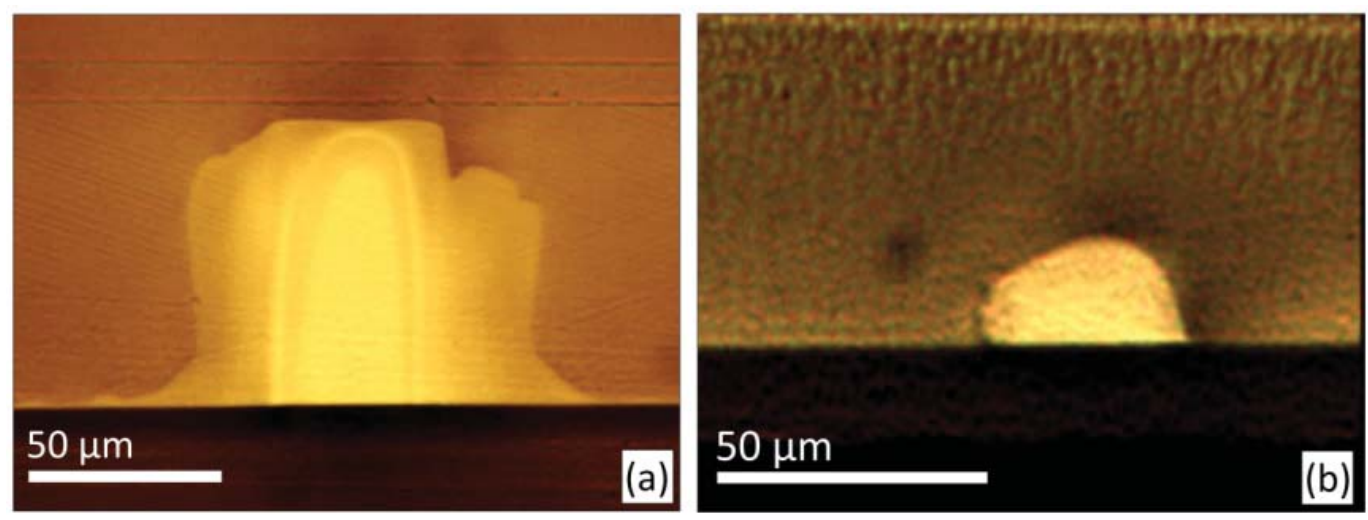

Figure 4.12 LDW WG abnormalities observed: high dwell time (a) and underexposure (b).

Over curing was observed at both high intensities and low writing speeds. At high intensities the material undergoes flash curing and polymerizes differently. While this usually results in a gradient-index profile, any unwanted optical properties are yet to be observed. At low writing speeds, typically $<10 \mathrm{~mm} / \mathrm{s}$, exposure in the regions from the writing beam's Gaussian tails initiates excess polymer debris that adheres to the WG sidewalls.

\subsection{2. $L D W M M$ \& SM WG Straights}

The numerous writing parameters controlled in the LDW process can not only affect overall WG shape, but also affect the WG's functionality and optical losses. Controlling the writing height, speed, and intensity to output the required energy to manufacture specified WG geometries, the LDW process can be optimized for both minimal fabrication time and optical loss. 
Optical losses for LDW MM WGs were measured with a result of $0.035 \pm 0.01 \mathrm{~dB} / \mathrm{cm}(\lambda$ $=830 \mathrm{~nm}$ ). Propagation losses were minimal $[0.025 \mathrm{~dB} / \mathrm{cm}]$ when the UV power was $>3$ $\mathrm{mW}$ and the writing velocity was $<50 \mathrm{~mm} / \mathrm{s}$. In comparison, MM WGs fabricated through photolithography exhibited losses of $0.03-0.05 \mathrm{~dB} / \mathrm{cm}(\lambda=830 \mathrm{~nm})[12,71]$.

SM WGs were also fabricated with the LDW process. Higher energy dose was required to write WGs within $6 \mu \mathrm{m}$ core layer. With a writing intensity of $8 \mathrm{~mW}$ and a writing speed of $30 \mathrm{~mm} / \mathrm{s}$, LDW SM WGs exhibited propagation losses of $0.55 \pm 0.065 \mathrm{~dB} / \mathrm{cm}(\lambda$ $=1310 \mathrm{~nm}$ ). In comparison, photolithographic results of SM WGs fabricated internally exhibited propagation losses of $0.59 \mathrm{~dB} / \mathrm{cm}(\lambda=1310 \mathrm{~nm})$. Photolithographic losses are slightly higher possibly due to the presence of mask-induced lithographic sidewall roughness.

Though spin-coating is the traditional method for applying uniform polymer coatings, alternative methods of material deposition would be required with the interest in largescale builds. Doctor blading [72] and ink-jet printing [73] can be integrated into the motion platform to uniformly coat appropriate substrates with WG material. LDW $50 \mu \mathrm{m}$ MM WG straights $25 \mathrm{~cm}$ in length were fabricated on FR-4 utilizing the doctor blading process [74] to coat both core and clad layers with measured optical losses as low as 0.04 $\mathrm{dB} / \mathrm{cm}$. Doctor blading has also been used to coat flexible polyimide substrates before patterning with LDW to form MM WGs performing at $0.031 \mathrm{~dB} / \mathrm{cm}$ [58]. 


\subsection{3. $L D W S M$ \& $M M W G$ Radial Bends}

Radial bends are essential in the implementation of polymer WGs in directional changes within optical interconnects. Bending losses are strongly dependent on the NA of the WG and may change due to large difference in UV curing intensity between the LDW [1-10 $\mathrm{kW} / \mathrm{cm} 2]$ and traditional photolithography $\left[10-30 \mathrm{~mW} / \mathrm{cm}^{2}\right]$. It is essential to evaluate radial losses to evaluate both curing effects from the LDW process and to determine optimal bend radii for minimal bend structure loss.

LDW MM and SM WG radial bends were fabricated and measured. After omitting propagation losses radial bend losses were compared to those fabricated via photolithography [75]. LDW MM and SM polymer WGs exhibited higher loss at shorter $(<6 \mathrm{~mm})$ radial bends in comparison to photolithographic builds, an effect that is more pronounced for SM WGs. MM and SM WGs fabricated through both methods still exhibit $<1 \mathrm{~dB}$ in radial losses at $5 \mathrm{~mm}$ radial bends and minimal radial losses at bends $>$ $6 \mathrm{~mm}$ in radius. Comparison of LDW and lithographic radial bend losses are shown in Figure 4.13.

The fact that LDW radial bends exhibit higher radial losses could be explained through a couple of theories. With a Gaussian writing beam, an extremely high intensity beam will cure the material differently over the profile of the WG cross-section. With most of the UV power focused at the WG center, the core boundary marginally cures due to lower UV levels. This porous boundary would result in cladding diffusion, intermixing at the core-clad boundary, and a graded-indexed RI profile. Furthermore, instantaneous 
polymer curing may decrease the core's optical density, reducing the WGs RI contrast and overall guiding capabilities [48].
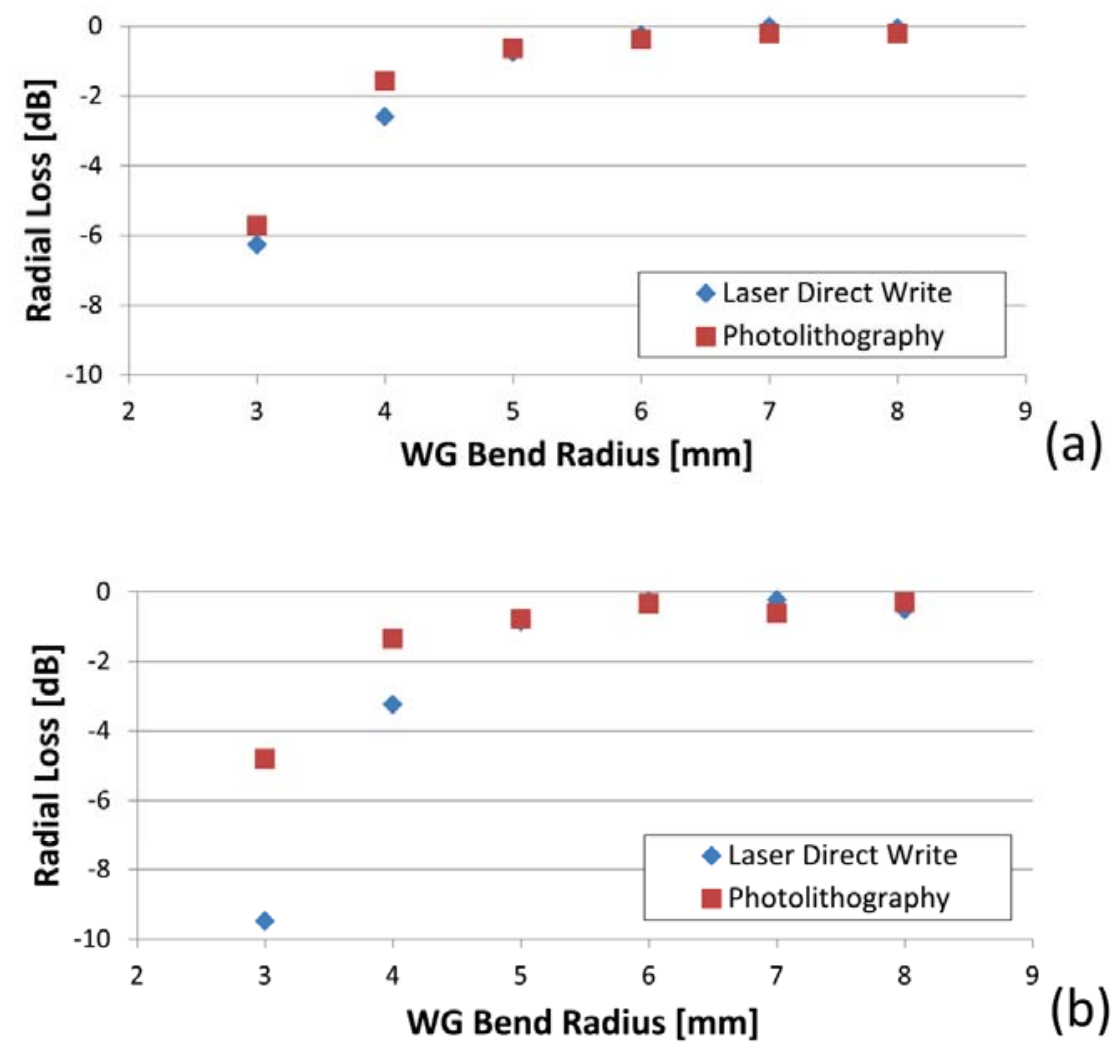

Figure 4.13 Radial bend loss for MMWGs $(\lambda=830 \mathrm{~nm})(\mathrm{a})$ and $S M W G s(\lambda=$ $1310 \mathrm{~nm})$ (b).

\subsubsection{LDWWG Crossings}

Optical WGs have the capability to intersect perpendicularly with minimal impact on optical loss and cross-talk. WG crossings allow for complex optical wiring boards to be 
manufactured on a single layer, reducing both material requirements and device complexity.

A main concern with LDW is the increase in UV energy delivered at the intersections of LDW WGs. Increasing the curing energy widens the WG widths and fillets the WG corners within the intersection. These rounded corners will effectively mix and leak the higher propagation modes in the optical signal. As shown in Figure 4.14 and Figure 4.15, both MM and SM WG crossings fabricated through LDW do exhibit radial curvature and some topological variations at the WG intersections.

Optical testing demonstrated that LDW MM and SM WG crossings exhibit optical losses of $0.04 \mathrm{~dB} /$ crossing $(\lambda=830 \mathrm{~nm})$ and $0.12 \mathrm{~dB} /$ crossing $(\lambda=1310 \mathrm{~nm})$ respectively. Photolithographic MM WG crossings have been stated in literature to exhibit losses between $0.006-0.01 \mathrm{~dB} /$ crossing depending on the amount of mode excitation permitted in the MM WGs [12]. Photolithographic SM WG crossings were fabricated internally and exhibited optical crossing loss of $0.02 \mathrm{~dB}$ /crossing. The LDW MM and SM polymer WG crossing losses are shown in Figure 4.16.

A large disconnect is observed when crossing losses are compared between the two fabrication procedures. LDW WG crossing loss is 4-6x higher than photolithographic builds due to overexposure effects at WG intersections. Adjustments to the LDW process would be required to minimize additional UV exposure at WG intersections if the fabrication of high-density optical WG builds with a high quantity of WG crossings is 
desired. The inclusion of a high-speed shutter would activate at intersections without disturbing the internal power stability or writing velocity of the LDW process.
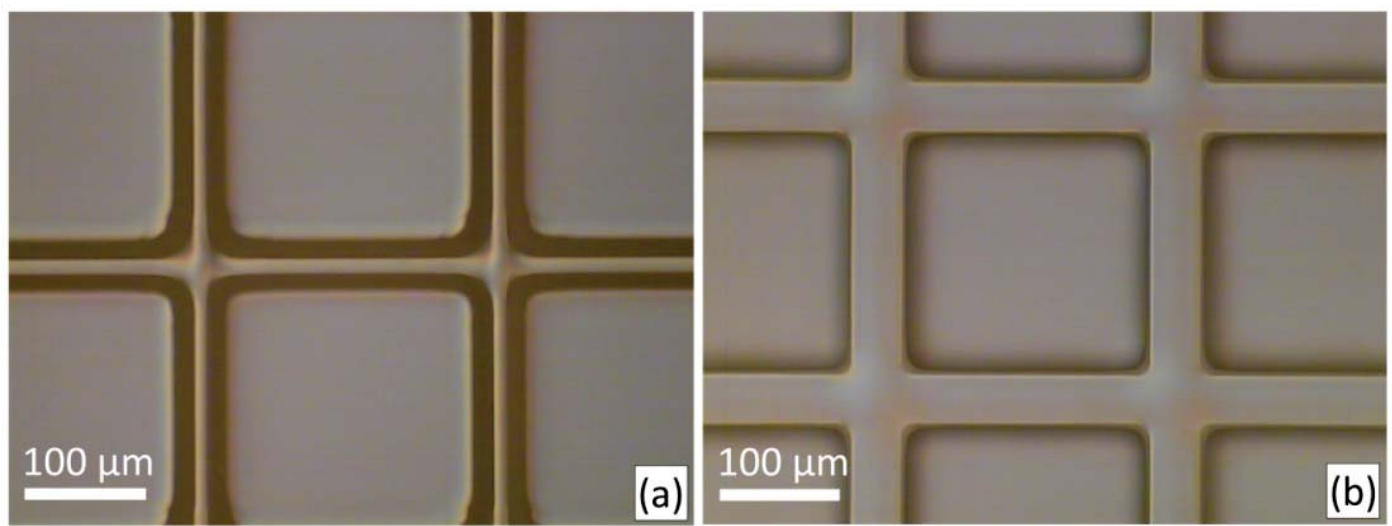

Figure 4.14 Top-down image of $M M W G$ crossings fabricated from $L D W(a)$ and photolithography (b).
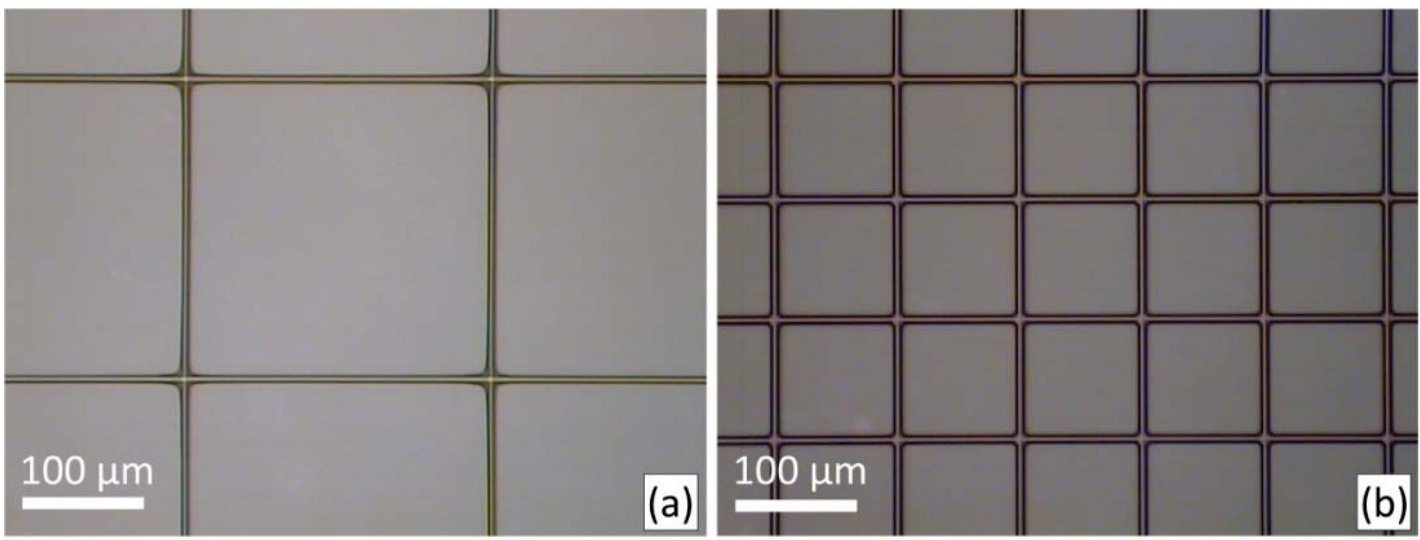

Figure 4.15 Top-down image of SM WG crossings fabricated from LDW (a) and photolithography (b). 

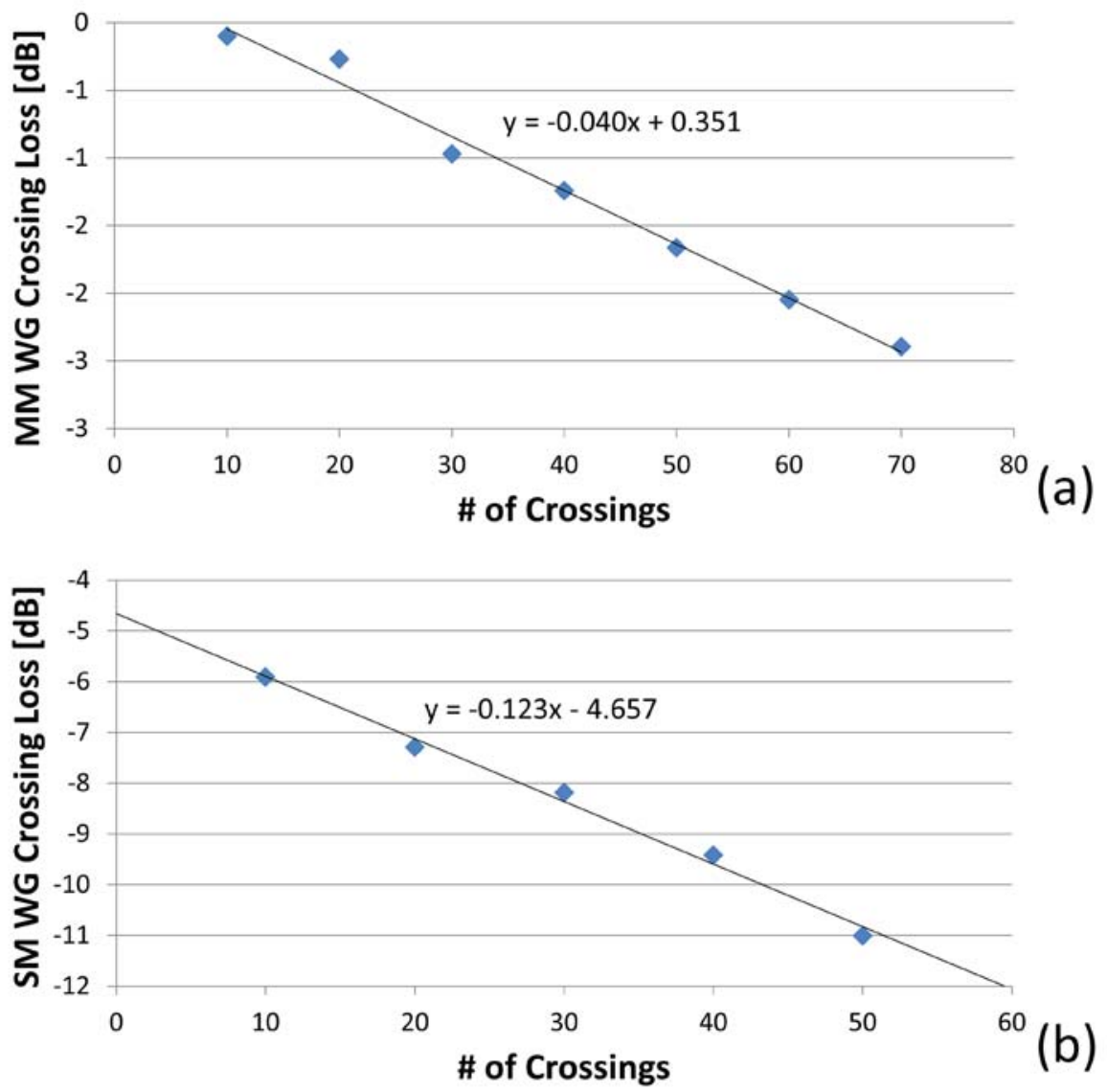

(b)

Figure 4.16 LDW WG crossing loss for MMWGs ( $\lambda=830 \mathrm{~nm}$ ) (a) and SM WGs $(\lambda=1310 \mathrm{~nm})(b)$.

\subsection{LDW Conclusion}

With new interest in integrating optical interconnects into large-scale printed wiring boards cost-effective fabrication methods are important for prototyping proof-of-concept devices before beginning large-scale manufacturing. The proposed LDW method with a flexible programmable interface is proven as a cost-effective prototyping method for fabricating UV-curable polymer SM and MM WGs with rigid rectangular structures, low 
loss, and high repeatability. A theoretical Gaussian beam model with $<5 \%$ error has been developed allowing for alterations in WG dimensions by controlling various writing parameters in the LDW process.

Optical testing confirmed that LDW MM WGs $(0.035 \mathrm{~dB} / \mathrm{cm}, \lambda=830 \mathrm{~nm})$ and LDW SM WGs $(0.55 \mathrm{~dB} / \mathrm{cm}, \lambda=1310 \mathrm{~nm}) \mathrm{WG}$ propagation losses are comparable to photolithographic MM WGs $(0.04 \mathrm{~dB} / \mathrm{cm}, \lambda=830 \mathrm{~nm})$ and SM WGs $(0.59 \mathrm{~dB} / \mathrm{cm}, \lambda=$ $1310 \mathrm{~nm}$ ). Optimal LDW WGs were obtained with writing powers $>3 \mathrm{~mW}$ and writing beam diameters $0.5-1.0 x$ the size of the desired WG width. LDW WG radial bends and crossings were also evaluated and shown to be highly comparable to photolithographic WGs, exhibiting $<1 \mathrm{~dB}$ radial losses at $5 \mathrm{~mm}$ radial bends and $0.04 \mathrm{~dB} /$ crossing $(\lambda=830$ $\mathrm{nm})$ and $0.12 \mathrm{~dB} /$ crossing $(\lambda=1310 \mathrm{~nm})$ for MM WGs and SM WGs respectively. 


\section{Chapter 5: SM WG S-Bends and Splitters}

Polymer waveguide S-bends are necessary for fan-out routing techniques and optical splitting in high density optical interconnects. Designing and manufacturing optimal Sbends is critical for minimizing optical link loss while maintaining overall size and layout constraints. Complete structural loss analysis is demonstrated theoretically and shown experimentally utilizing both radial and transitional loss in SM polymer WG radial-arc, cosine, and raised-sine S-bend profiles.

Photolithographic SM WG S-bends were fabricated and measured at different dimensions. Structure loss was evaluated using theoretical bend loss models. Trade-off between WG S-bend structure loss and propagation loss for optimal device size is discussed. SM WG S-bends were incorporated into optical splitters to evaluate device loss and splitting ratios. WG tapers were incorporated into WG splitters and demonstrated to improve splitting efficiency and reducing footprint requirements.

1. Research in this chapter has been previously published by the author in the following references:

- K. L. Kruse and C. T. Middlebrook, "Fan-out routing and optical splitting techniques for compact optical interconnects using single-mode polymer waveguides," Journal of Modern Optics, pp. 1-10, 2014.

- K. Kruse and C. Middlebrook, "Laser-direct writing of single mode and multi-mode polymer step index waveguide structures for optical backplanes and interconnection assemblies," Photonics and Nanostructures-Fundamentals and Applications, 2014. 
LDW parameters required for efficient fabrication polymer WG S-bends were also determined. Segmented and smooth radial arc SM polymer WGs are realized through user-defined programs and compared experimentally. Cosine and raised-sine S-bends are realized through a segmentation method where the optimal incremental step length and bend dimensions are controlled to achieve minimal structure loss. LDW S-bends losses are compared with photolithographic S-bend results and explained using theoretical bend loss models. Fabrication results show that LDW is a viable method in the fabrication of advanced polymer waveguide structures.

\subsection{Introduction}

Polymer WGs must be designed for low propagation loss while exhibiting low structural loss when guided around bends and curves for implementation as optical interconnects. SM polymer WG designs can be designed to be geometrically constrained to the application's required foot-print. S-bends are curved WG designs that efficiently connect laterally offset WGs with low structure loss [30]. By applying both radial and transitional loss theory discussed in Chapter 2, polymer WG S-bend structures can be optimally designed to minimize device loss and maximize optical bandwidth density.

S-bends are optimal for many specific WG integrated devices in addition to WG routing. WG S-bends structures are utilized by designers to implement pitch control, optical coupling, and compact optical interconnect layouts. S-bends can adjust waveguide density within an optical data bus and be integrated in optical splitters and couplers which are important components in integrated Mach-Zehnder Interferometers (MZIs). Both S- 
bend designs are shown in Figure 5.1. The numerous applications for S-Bends include optical interconnects [29, 76], optical signal modulation [77], environmental sensing [78], and silicon-organic hybrid interconnects [79].

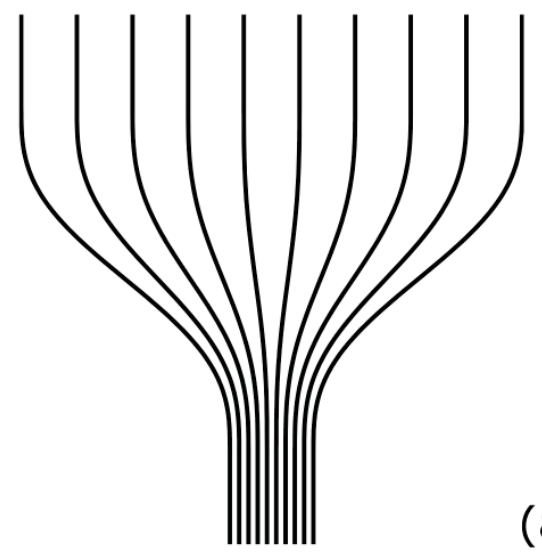

(a)

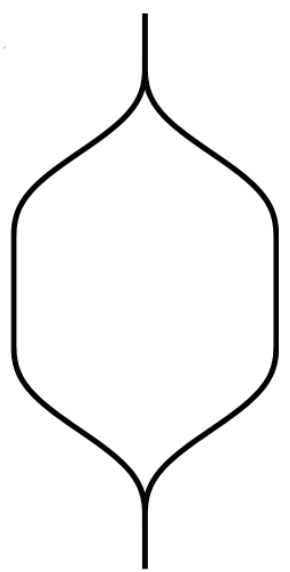

(b)

Figure 5.1 Images of S-Bend Utilized in WG fan-out (a) and Mach-Zehnder interferometer (b) designs.

Previous published journal articles have proposed complex solutions for minimizing optical loss while obtaining high optical density with SM WG S-bend structures including polyline-based S-bends [24] and radial tapers [25]. Polyline-based S-bends and radial tapers are convoluted and challenging structures to design and scale for integration into photolithographic masks. Other publications $[26,27,80]$ have shown that sinederived S-bends of equivalent dimensions offer similar levels of loss in simple, flexible, and expandable formulas. Many of the previous works cited are purely theoretical without any experimental results to verify and compare different S-bend designs. 
Other articles on S-Bend designs have focused solely on radial loss and have not incorporated transitional loss into total structure loss $[26,27]$. Transitional loss is a large factor in determining optical loss budgets in WG S-bend builds which increases as modal confinement in the WG core decreases. S-bend designs utilizing low numerical aperture (NA), step-index polymer WGs must account for both radial loss and transitional loss for accurate structure loss estimation.

WG S-bends have been previously fabricated using a variety of materials. Semiconductor-based devices have been fabricated through MBE-grown epilayering [28] and etching [29]. Titanium-doped Lithium Niobate S-bends have been fabricated, tested, and correlated to theoretical losses [30, 31]. Experimental results for polymer-based SM WG S-bends have only been reported using the radial arc design with an emphasis on cross-talk measurements [32]. Prior journal articles assume only one type of S-bend design for builds and do not discuss optimal S-bend profiles required for high degrees of integration density.

In this Chapter, total structure losses of SM polymer WG S-bends are evaluated using theoretical equations and rigorous simulations to determine optimal designs, configurations, and dimensions. The impact of the chosen NA upon the overall link loss of SM polymer WGs is also determined for varying S-bend structure designs. To confirm structure losses step-index SM WG S-bends were fabricated with various dimensions and correlated to theoretical losses. Optical splitters designed using SM WG S-bends and adiabatic tapers were also evaluated and fabricated to determine optimal splitting 
efficiencies. SM WG S-bends were also fabricated through LDW and experimentally compared to photolithographic S-bend loss. Finally, a brief discussion of the tradeoff between optimal loss and minimal package foot-print requirements for both devices follows.

\subsection{Design and Theoretical Loss}

Sine-derived S-bends are designed from one of three profiles: radial arc, cosine, and raised-sine S-bends. These profiles can easily be expanded to a designated width, $W$, and length, $L$. Radial arc S-bends are formed using two inverted arcs of constant curvature, calculated by Equation (5.1), that intersect at the arcs' tangents. Cosine and raised-sine Sbends are formed using the sinusoidal functions stated in Eq. (5.2) and (5.3) respectively. These profile's functions and their associated curvature function, the inverse of $R(s)$, are both expressed in Figure 5.2 and Figure 5.3.

$$
\begin{aligned}
& y(x)=\left\{\begin{array}{ll}
\sqrt{R^{2}-x^{2}}-(R-W), & \text { where } 0 \leq x \leq L / 2 \\
-\sqrt{R^{2}-(x-L)^{2}}+R, & \text { where } L / 2 \leq x \leq L
\end{array}, \quad R=\frac{L^{2}}{4 W}\left(1+\frac{W^{2}}{L^{2}}\right)\right. \\
& y(x)=\frac{W}{2}\left(1-\cos \left(\frac{\pi}{L} x\right)\right), \quad \text { where } 0 \leq x \leq L \\
& y(x)=\frac{W}{L} x-\frac{W}{2 \pi} \sin \left(\frac{2 \pi}{L} x\right), \quad \text { where } 0 \leq x \leq L
\end{aligned}
$$


According to the theoretical work done by Mustieles [26] and Kumar [27], cosine Sbends have the lowest losses followed by radial arc and then raised sine S-Bends.

However, their work is based purely off of radial losses alone and fails to integrate the transitional losses present at the S-bend interfaces. As most S-bends are directly connected to WG straights in practical applications transitional losses play a large role in the S-bend's total structure loss. A complete loss analysis of the overall S-bend structure including the impact from both radial and transitional loss is needed.

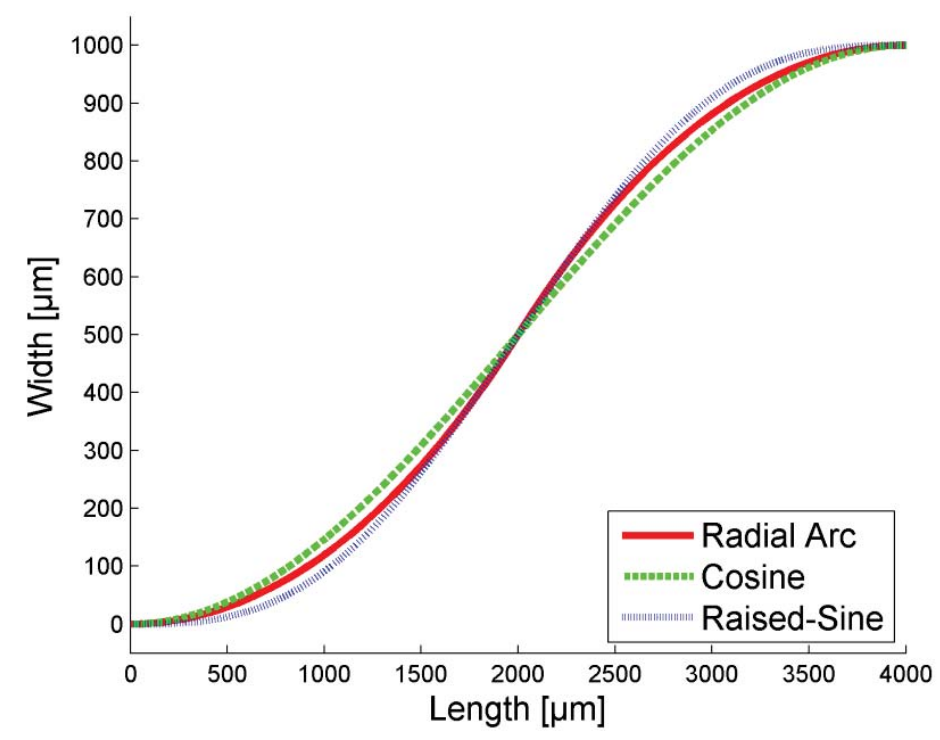

Figure 5.2 S-Bend profile functions.

The low RI contrast utilized in SM WGs to minimize coupling loss with optical fibers decreases the amount of modal confinement. This increases the amount the propagating mode shifts outwards within WG bends. Radial arc and cosine S-bends both suffer these heightened levels of transitional loss at their end-faces. Radial arc S-bends also suffer 
transitional loss at its midsection between its inverted radial arcs. Raised-sine S-Bends experience no regions of modal mismatch due to its smooth curvature profile and zero curvature at the end-faces, $y(0)$ and $y(L)[31]$.

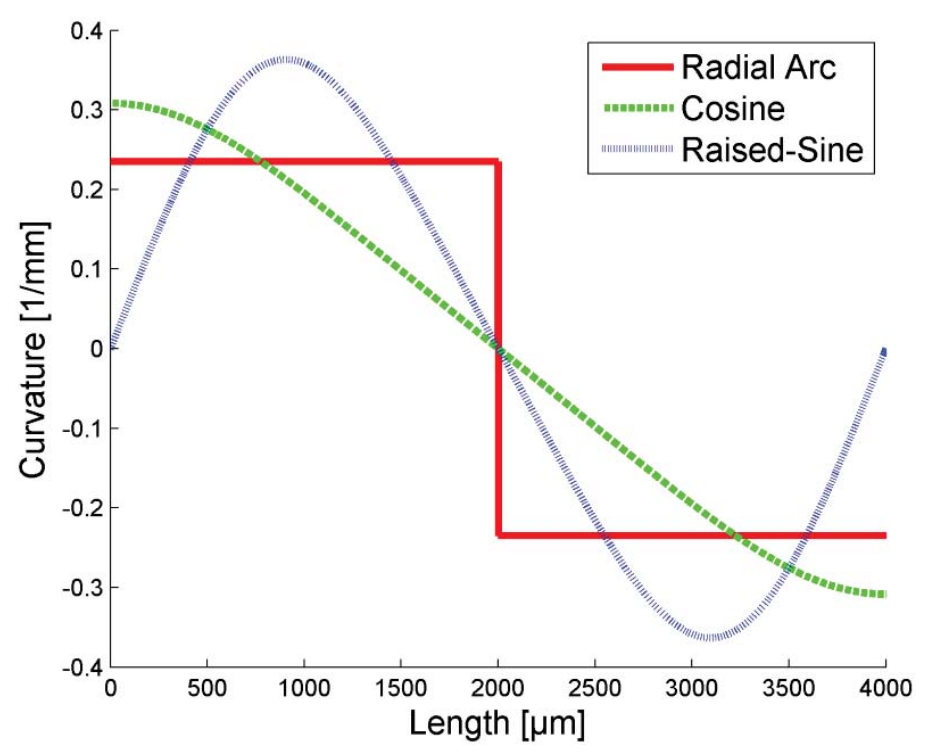

Figure 5.3 S-Bend curvature functions.

Total structure loss of SM polymer WG S-bends is evaluated using theoretical equations and rigorous simulations to determine optimal S-bend configurations and dimensions. The Beam Propagation Method (BPM) was used to simulate the radial and transitional losses, both as a function of radius of curvature, for symmetric WGs of various NA and size that fulfill SM functionality. These values were then utilized to solve for the total structure loss for the three S-bend designs: radial arc, cosine, and raised-sine.

S-bend structure loss was simulated for SM WGs of varying NA $(0.15-0.35)$ to show Sbend efficiency for various SM WG characteristics. To maintain SM functionality, the 
dimensions of the SM WGs of various NAs were calculated using the effective index method [48]. Simulated losses for S-bend structures of various widths, lengths, and NAs are shown in Figure 5.4 and Figure 5.5.

Raised-sine S-bends exhibit the shortest length requirements to obtain minimal $(<0.1 \mathrm{~dB})$ structure loss regardless of the WG's NA. However, there is negligible difference in structure loss when comparing S-bend designs in this range. S-bend design selection is more important when footprint requirements are smaller than allowed for minimal structure loss. Reducing length requirements, the most efficient S-bend designs are cosine and radial arc S-bends for low NA and high NA WGs respectively.

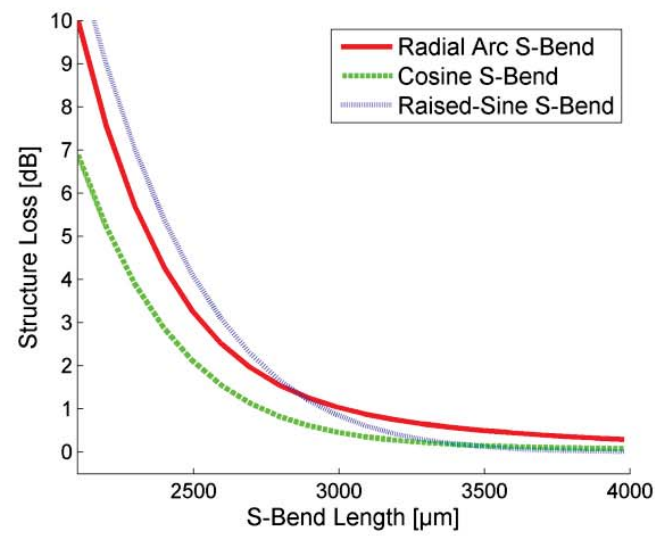

(a)

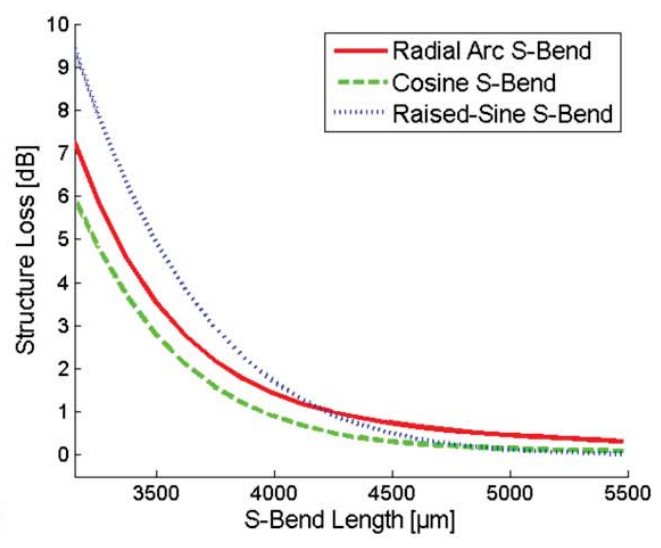

(b)

Figure 5.4 Simulated SM WG $(N A=0.15)$ structure loss for S-Bend with a 0.5 $\mathrm{mm}(\mathrm{a})$ and $1.0 \mathrm{~mm}$ (b) S-bend width. 


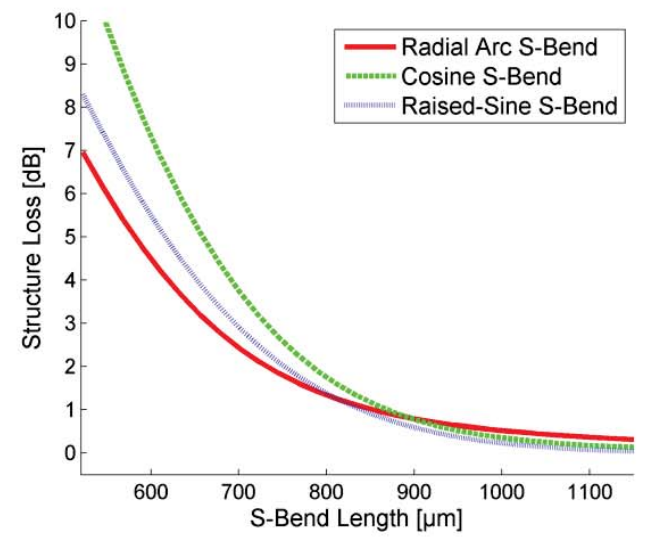

(a)

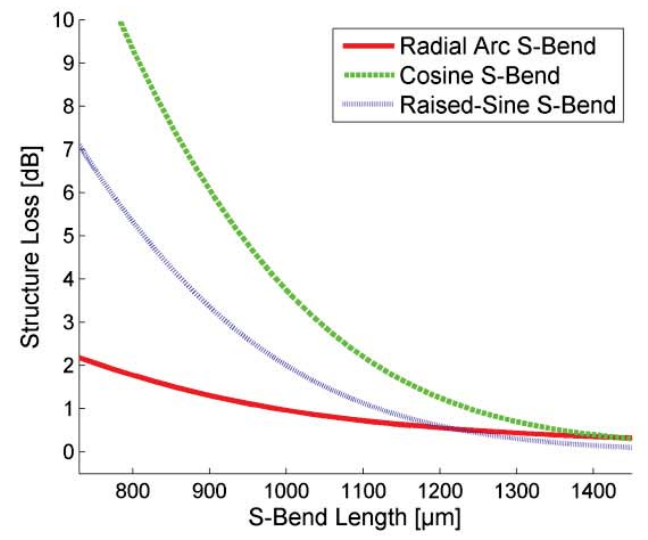

(b)

Figure 5.5 Simulated SM WG $(N A=0.35)$ structure loss for $S$-Bend with a 0.5 $\mathrm{mm}$ (a) and $1.0 \mathrm{~mm}$ (b) $S$-bend width.

Since the LDW platform utilizes two linear stage drive motors to move the sample only radial arc S-bends can be easily programmed using clockwise (G2) and counterclockwise (G3) functions to write the two intersecting radial bends of constant curvature described by Equation (5.1). Fabricating complex radially-varying high-density optical interconnects including S-bends using the LDW method is not readily implemented through pre-defined drive command routines in G-code that drives the two-axis writing stage commands [81]. Indirect writing methods must be applied for efficient implantation of LDW complex WG designs utilizing industrial-recognized mechanical programs (Gcode). LDW cosine and raised-sine polymer WGs S-bends can alternatively be implemented using segmentation, as illustrated in Figure 5.6. A complex polyline can be equally divided into multiple linear segments of equal segmentation length $\left(L_{s}\right)$ to approximate the final structure and reduce computational requirements. S-bends are 
segmented into incremental XY translations that are sequentially written using linear (G1) operations to complete the structure.

\subsection{S-Bend Fabrication}

Photolithographic S-bends fabricated from $6 \mu \mathrm{m}$ SM polymer WGs were desired for experimentally evaluating theoretical bend loss models. Radial arc, cosine, and raisedsine S-Bends were fabricated at various widths [500-1000 $\mu \mathrm{m}]$ and length [2000-6000 $\mu \mathrm{m}]$ with straight lead-in and lead-out sections to incorporate transitional losses that would be present in a workable design. Optical splitters were manufactured using Sbends and tapers. Fabricated WG straights, S-bends, and splitters are shown in Figure 5.7. The structure losses for S-bends and splitters were evaluated after omitting the known propagation loss $(0.6 \mathrm{~dB} / \mathrm{cm})$ previously calculated from the cutback method.

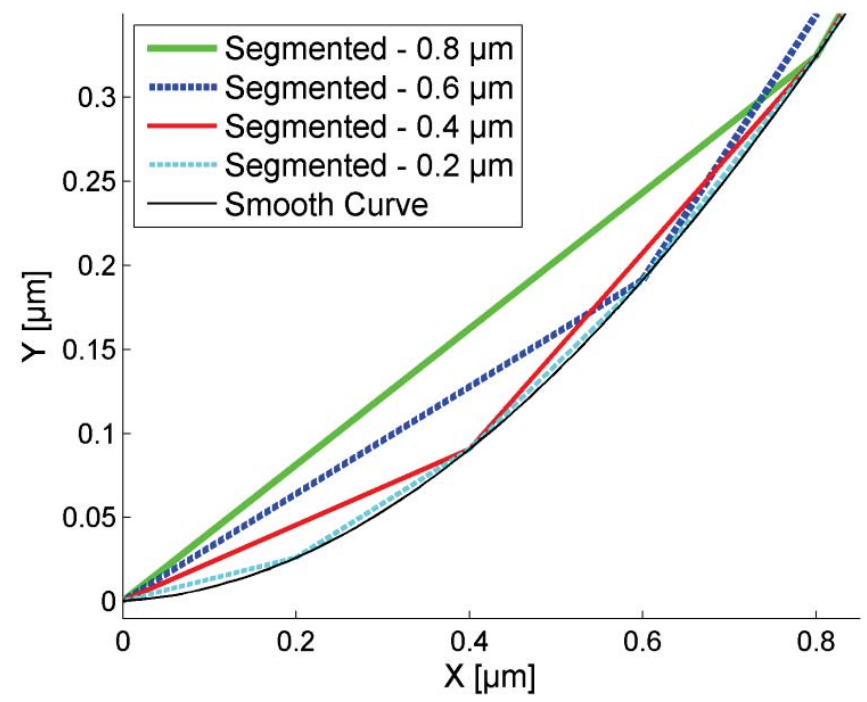

Figure 5.6 Illustration of segmentation utilized in realizing a sinusoidal bend. Shorter segmentation lengths approach the ideal line of the smooth bend. 

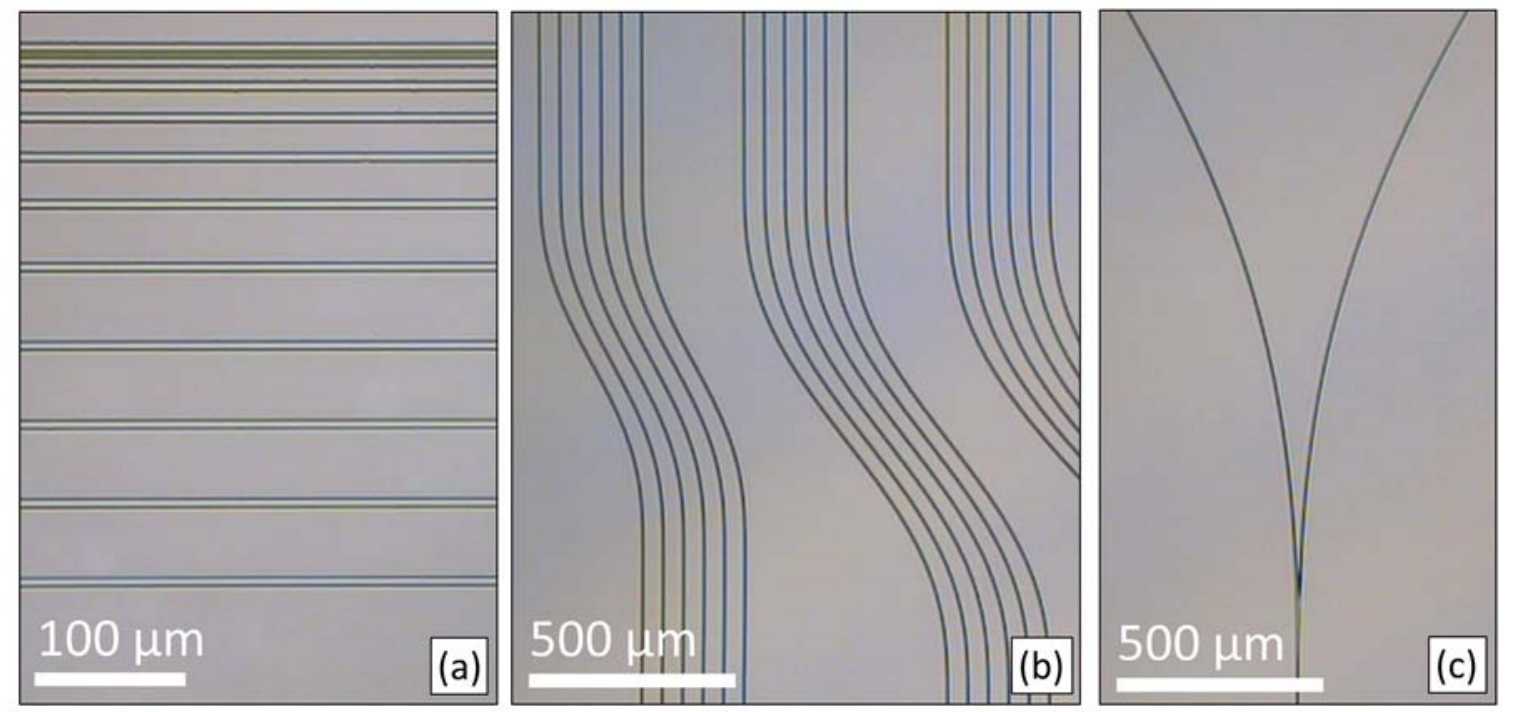

Figure 5.7 Top-down view of $6 \mu \mathrm{m}$ SM WG straights (a), raised-sine S-bends (b), and splitters (c).

Polymer SM WG straights and S-bends were manufacturing utilizing the LDW method with a UV power of $4 \mathrm{~mW}$ at $25 \mathrm{~mm} / \mathrm{s}$ on cleaned silicon substrates. LDW radial arc Sbends were written utilizing linear $(\mathrm{G} 1)$ and radial $(\mathrm{G} 2, \mathrm{G} 3)$ operations. Radial arc, cosine, and raised-sine S-bends were manufactured utilizing the segmentation method with various segmentation lengths (G1). The structure loss of LDW SM WG S-bends was also calculated through similar methods. 


\subsection{Photolithographic Results}

\subsubsection{Photolithographic SM WG S-Bends}

Raised-sine S-bends theoretically exhibit no transitional losses, shown previously in Figure 5.2, and can be utilized to find the radial loss coefficients for fabricated SM polymer WGs. Raised-sine S-Bend radial loss was isolated from experimental losses after omitting WG propagation and SMF coupling loss. Theoretical loss curves showed strong correlation when fit to experimental results, as shown in Figure 5.8. Fitted theoretical curves resulted in radially-independent loss coefficients from Eq. (2.10) of $C_{l}=0.053$ [unitless $]$ and $C_{2}=2.3 \mathrm{~mm}^{-1}$.

Knowing the radial loss constants the transitional loss coefficient for S-bend structures can now be isolated. Radial arc, cosine, and raised-sine S-bends were fabricated and tested to compare structure loss, shown in Figure 5.9. Fitted theoretical curves for radial arc and cosine S-bends gave a transitional decibel loss coefficient from Eq. (2.14) of $T_{1}=$ $1.6 \mathrm{~mm}^{2}$. 


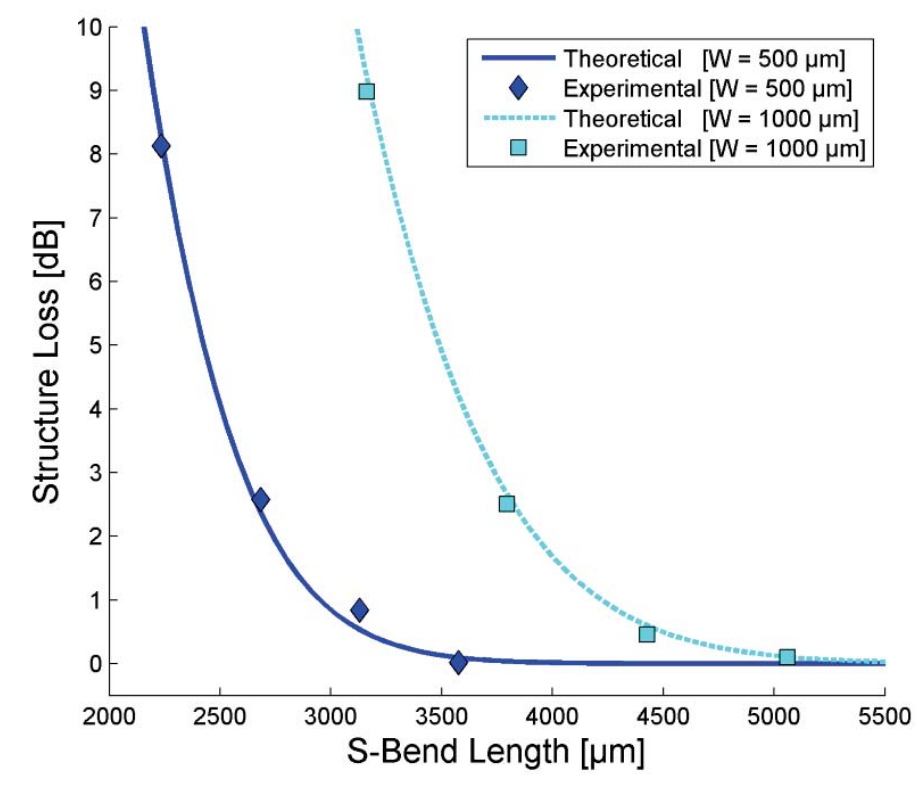

Figure 5.8 Raised-sine S-Bend structure loss shown using theoretical curves (lines) and experimental results (markers).

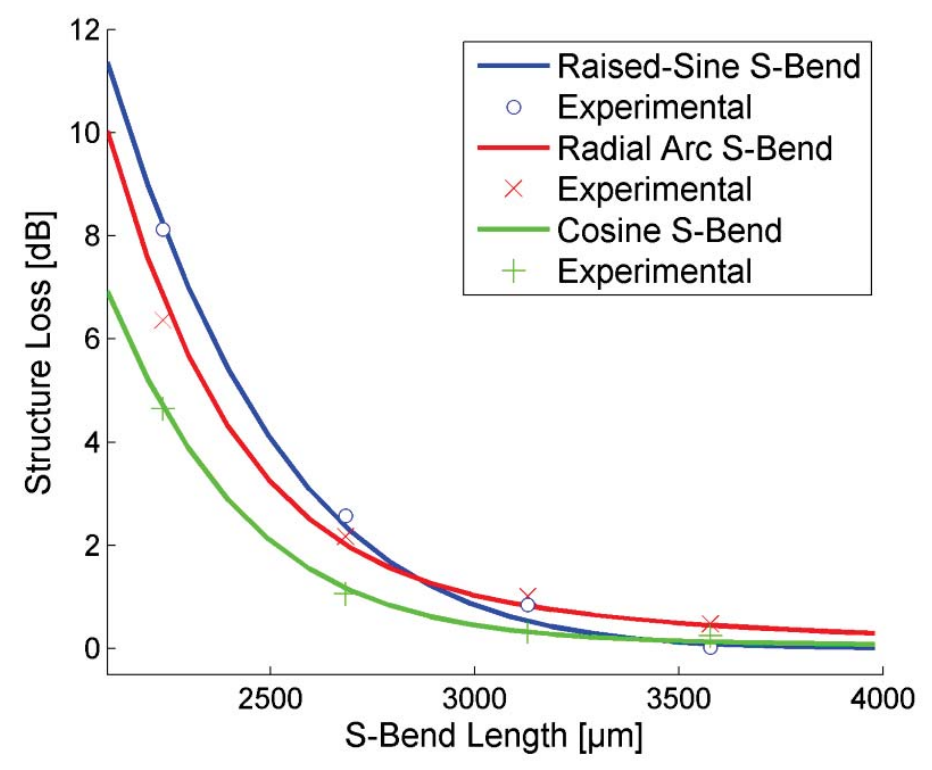

Figure 5.9 S-Bend structure loss for different S-bend designs shown using theoretical curves (lines) and experimental results (markers). 
The structure loss for all three devices are quite comparable while still exhibiting loss characteristics based on the ratio of radial-to-transitional loss present in each structure. Raised-sine s-bends structure losses are solely dependent on exponential-based radial loss. While the raised-sine profile is the least efficient design at shorter S-bend lengths, its structure loss curve approaches minimal loss at the fastest rate as the S-bend length increases. In contrast, the radial arc design heavily relies on the quadratically-dependent transitional loss and exhibits higher levels of structure loss at longer S-bend lengths. Cosine S-bends exhibit the lowest loss at shorter S-bend lengths since this design balances radial and transitional loss, both which increase nonlinearly with decreasing bend radius.

\subsubsection{S-Bend Splitters}

Optical splitters utilize S-bends for compact designs for both power distribution channel monitoring and MZIs. While optical splitters can be manufactured in the form of a Ybranch the small-angle requirement $\left(<2^{\circ}\right)$ for efficient light division requires a long splitter length to obtain minimal splitting loss [82]. Utilizing S-bends for optical splitting significantly reduces the required footprint of the splitter.

Optical splitters utilizing shortened $1 \mathrm{~dB}$ and $3 \mathrm{~dB}$ loss raised-sine S-bends were fabricated and measured. Optical testing of photolithographic splitters showed balanced levels of optical power splitting with a measured splitting ratio of 49:51 $\pm 2 \%$. Straight WG-to-S-bend splitters exhibited $>3 \mathrm{~dB}$ of splitting loss not accounting propagation loss or S-bend structure loss. Splitting loss is due to S-bend overlapping and WG widening 
before optical splitting occurs. Not only does this cause a discontinuity in the radius of curvature, introducing transitional loss, but it also starts the S-bend at an offset angle. Longer S-bends would result in lower levels of overlap discontinuity and lower splitting loss.

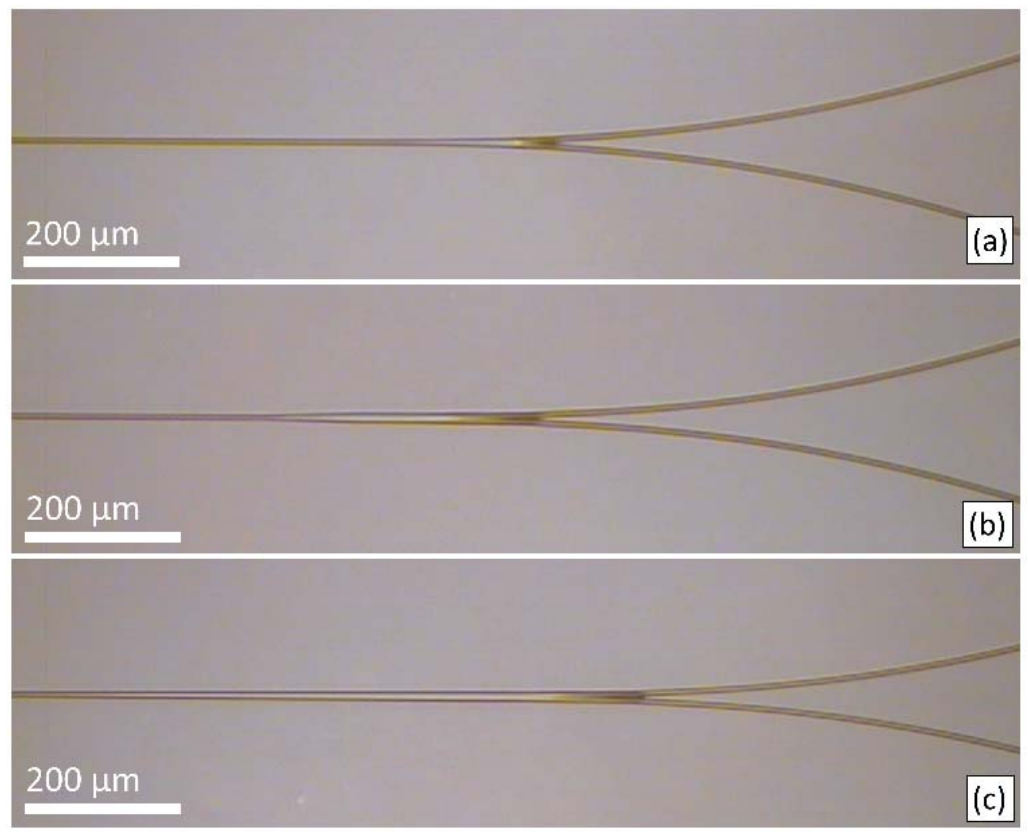

Figure 5.10 Top-down view of WG S-bend splitters integrated with no splitting taper (a), a $100 \mu \mathrm{m}$ long taper (b), and a $500 \mu \mathrm{m}$ long taper (c).

Adiabatic tapers can be utilized as an alternative method to improve splitting efficiencies while minimizing device size. Splitting tapers $100 \mu \mathrm{m}$ and $500 \mu \mathrm{m}$ long were inserted between the straight/S-bend interfaces, as shown in Figure 5.10. The taper widens the fundamental mode before coupling with S-bend splitters. This modal expansion removes unwanted S-bend overlap, reduces transitional losses, and minimizes overall splitting 
loss. The experimental splitting losses for the taper designs are shown in Figure 5.11. The inclusion of $100 \mu \mathrm{m}$ and $500 \mu \mathrm{m}$ long tapers in S-bend splitters reduced splitting loss by $1.9 \mathrm{~dB}$ and $2.1 \mathrm{~dB}$ respectively. Theoretical BPM simulations exhibit similar patterns in loss reduction as shown in Figure 5.12. While a similar difference $(0.2 \mathrm{~dB})$ in splitting loss is observed between $100 \mu \mathrm{m}$ and $500 \mu \mathrm{m}$ taper lengths experimental results exhibit higher splitting loss in comparison to theory. Residual polymer is present within the splitting region, shown in Figure 5.13. Due to the S-bend overlap present in splitters without tapers optical scattering has a stronger effect on splitting loss due to its adhesion to $\mathrm{WG}$ regions of higher curvature.

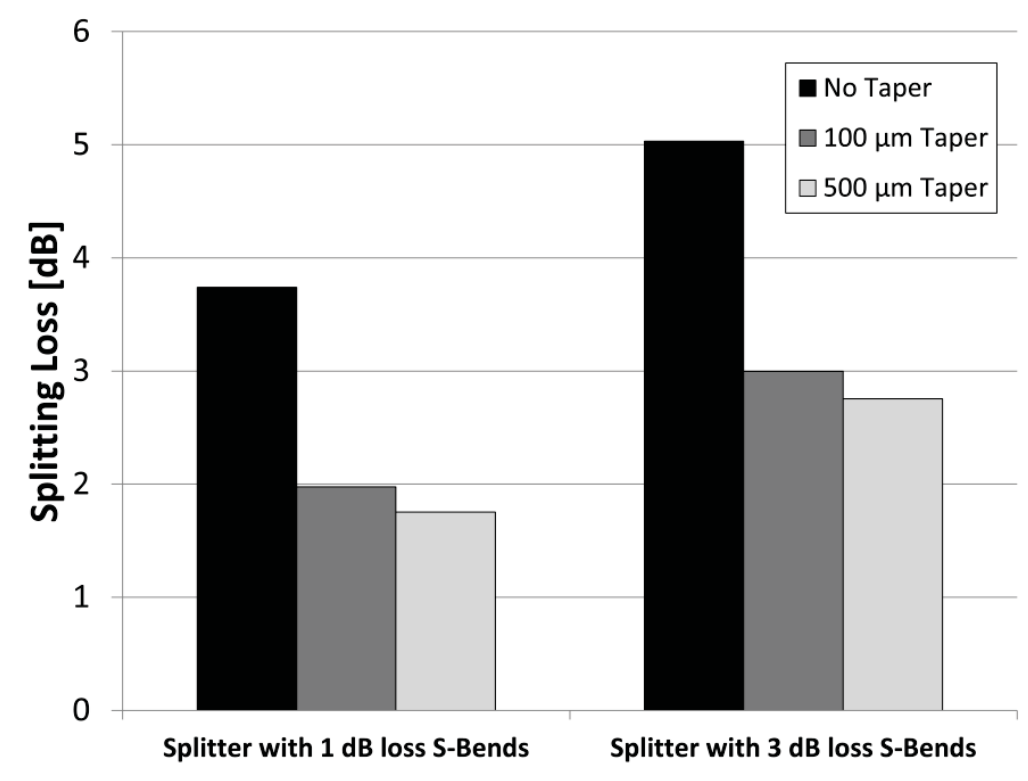

Figure 5.11 Experimental splitting loss as a function of S-bend type and taper length. 


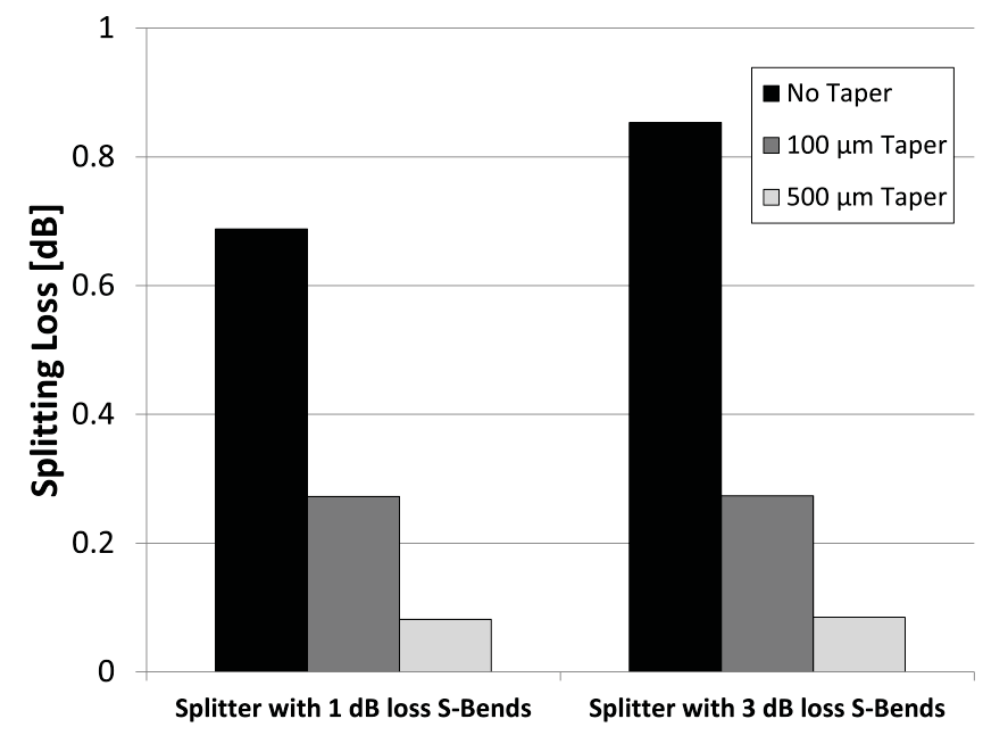

Figure 5.12 Theoretical splitting loss as a function of S-bend type and taper length.

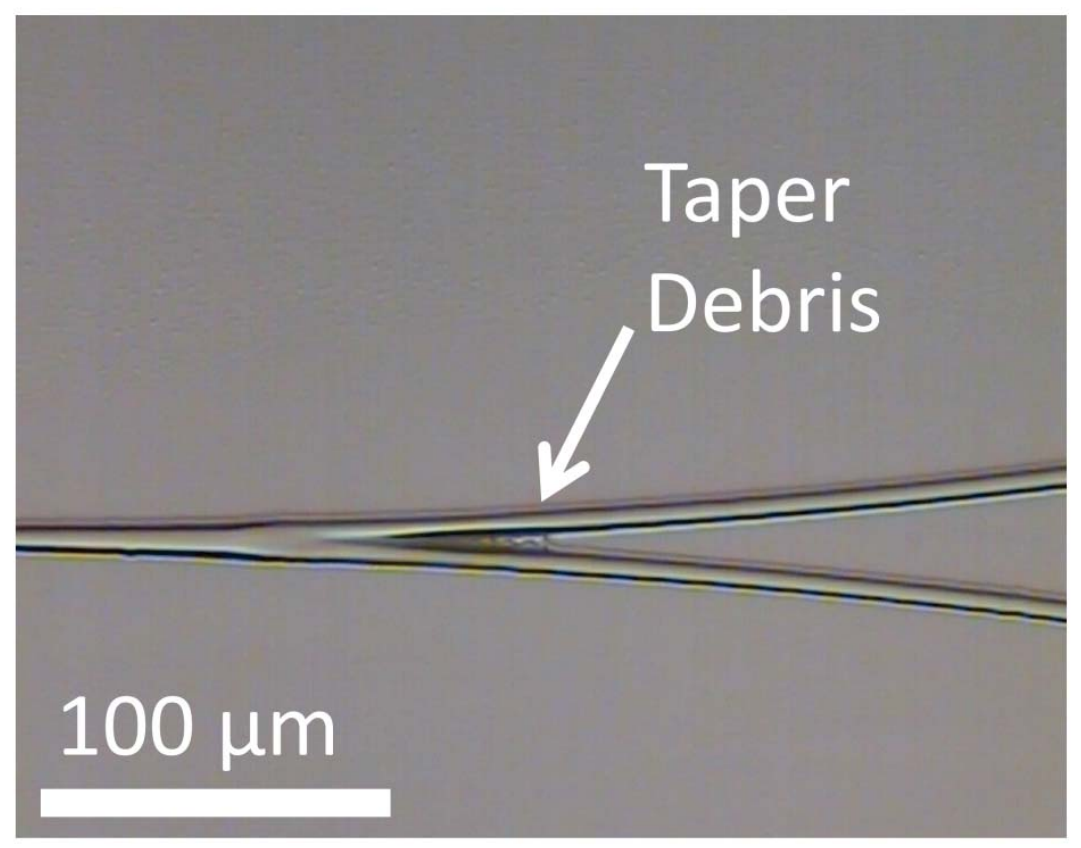

Figure 5.13 Residual polymer material present within an S-bend splitter. 


\subsubsection{Laser Direct Written SM WG S-Bends}

The chosen segmentation length $\left(L_{s}\right)$ in LDW complex polymer WG bends is important in the fabrication of low loss WG bends. A minimum segmentation length allows for minimum tilt angles to reduce the transitional loss at polymer WG corner bends [82, 83]. Decreasing segmentation length results in an upper limit where the translation stage cannot keep up with the small-step linear operations. Beyond this limit the LDW process will incur increasing levels of position and velocity error resulting in irregular WGs. Writing irregularities can be visually observed when the segmentation length is not optimally chosen, as seen in Figure 5.14.
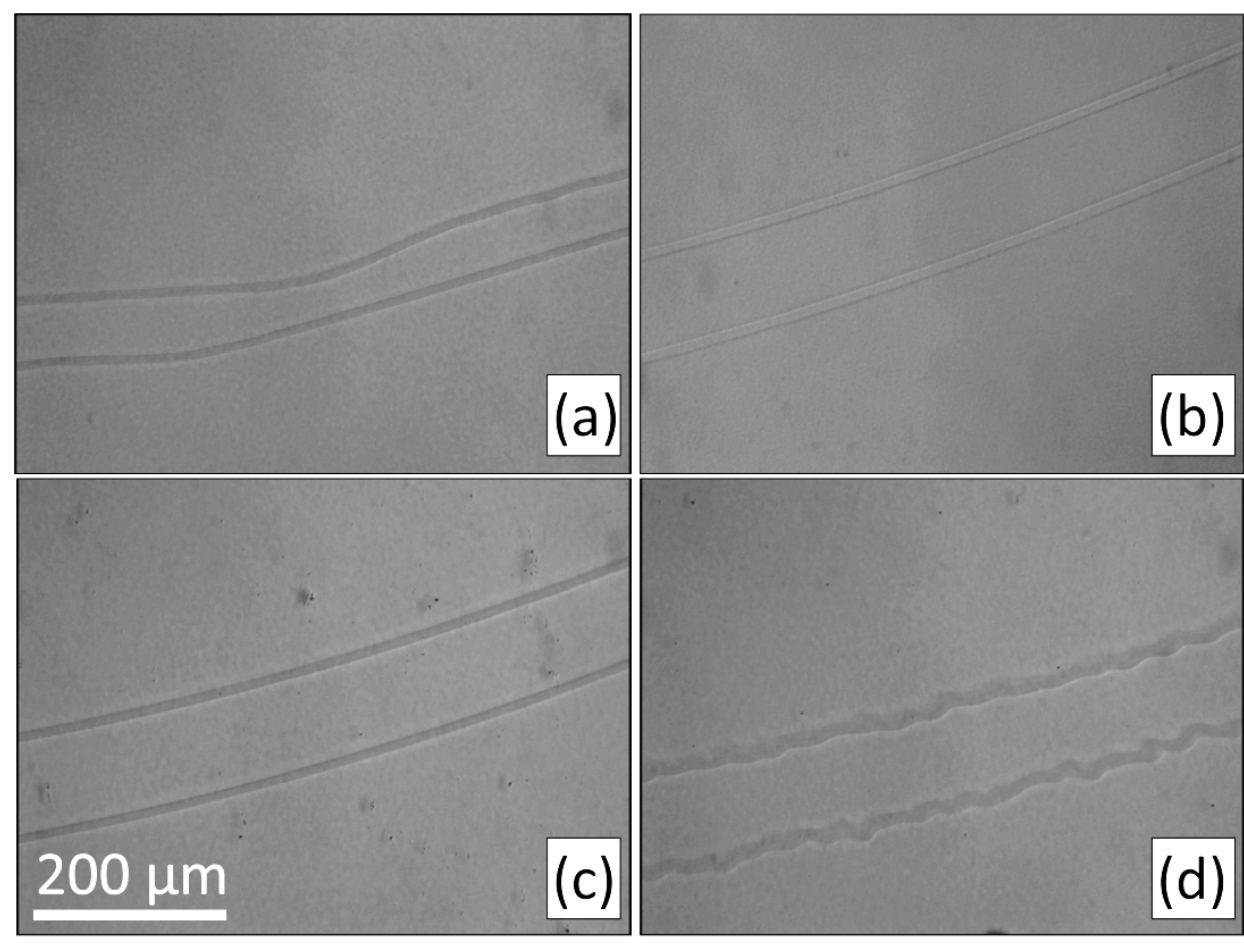

Figure 5.14 Cosine SM WG S-bends with segmentation lengths of $1000 \mu \mathrm{m}$ (a), $100 \mu \mathrm{m}(\mathrm{b}), 10 \mu \mathrm{m}(\mathrm{c})$, and $1 \mu \mathrm{m}(\mathrm{d})$. 
Optimal segmentation length to produce smooth polymer WG bends is highly dependent on the writing speed and computational time of the LDW platform. S-bend loss as a function of segmentation length is shown in Figure 5.15. SM WG S-bend structure loss was minimal with a segmentation length of 10-50 $\mu \mathrm{m}$ when written at $25 \mathrm{~mm} / \mathrm{s}$. Segmentation lengths outside this region resulted in drastically increasing structure loss.

Long segmentation lengths resulted in large tilt-angle corner bends and translation noise, noticeable as audible bangs during the LDW process, as the platform attempts sudden changes in XY linear translation direction while maintaining a constant directional velocity. Short segmentation lengths caused translational shearing due to underdampened and limited driver response time resulting in audible grinding during the LDW process.

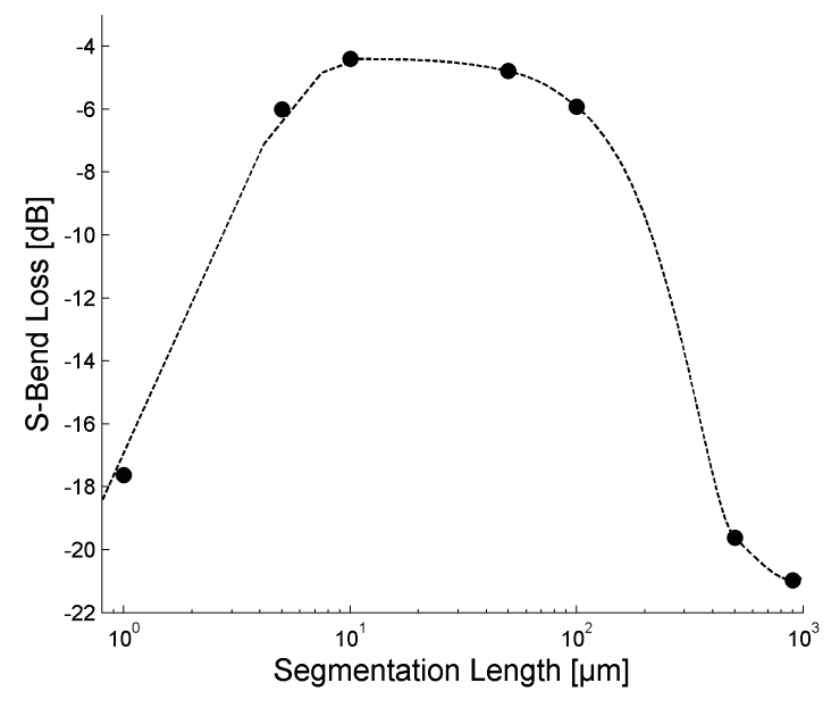

Figure 5.15 Experimental loss of SM WG cosine S-bends written at $25 \mathrm{~mm} / \mathrm{s}$ utilizing various segmentation lengths $(\lambda=1310 \mathrm{~nm})$. 
Radial arc S-bends manufactured using radial operations (G2, G3) and the segmentation method $\left(\mathrm{G} 1, L_{s}=20 \mu \mathrm{m}\right)$ were both fabricated to compare both fabrication method. Structure loss of both radial arc S-bends as a function of S-bend length is shown in Figure 5.16. Experimental results show no discernable difference in structure loss between Sbends fabricated through smooth radial bends and optimized linear segmentation.

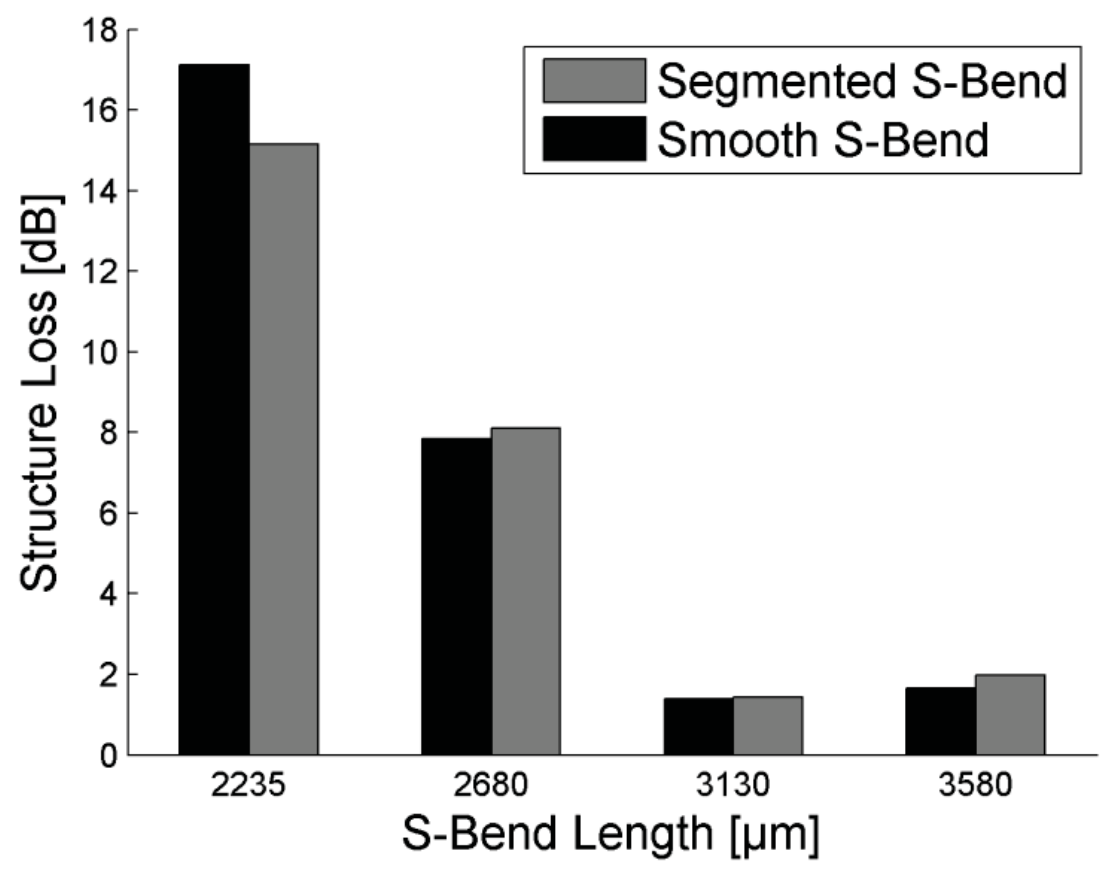

Figure 5.16 Experimental loss of SM WG radial arc consecutive and segmented $S$ bends $(\lambda=1310 \mathrm{~nm})$.

In order to fabricate raised-sine and cosine S-bends function segmentation is required in efficiently designing complex S-bend structures using G-code. Radial arc, cosine, and raised-sine S-bends are fabricated using the segmentation method $\left(L_{s}=20 \mu \mathrm{m}\right)$. The experimental structure loss of polymer WG S-bends fabricated through LDW is shown in 
Figure 5.17. Comparing these results to photolithographic builds shown in Figure 5.9 raised-sine S-bends present a very comparable curve profile between photolithographic and LDW measurements. LDW cosine S-bends exhibit slightly higher levels of structure loss. A large discrepancy is observed for radial arc S-bends between photolithography and LDW.

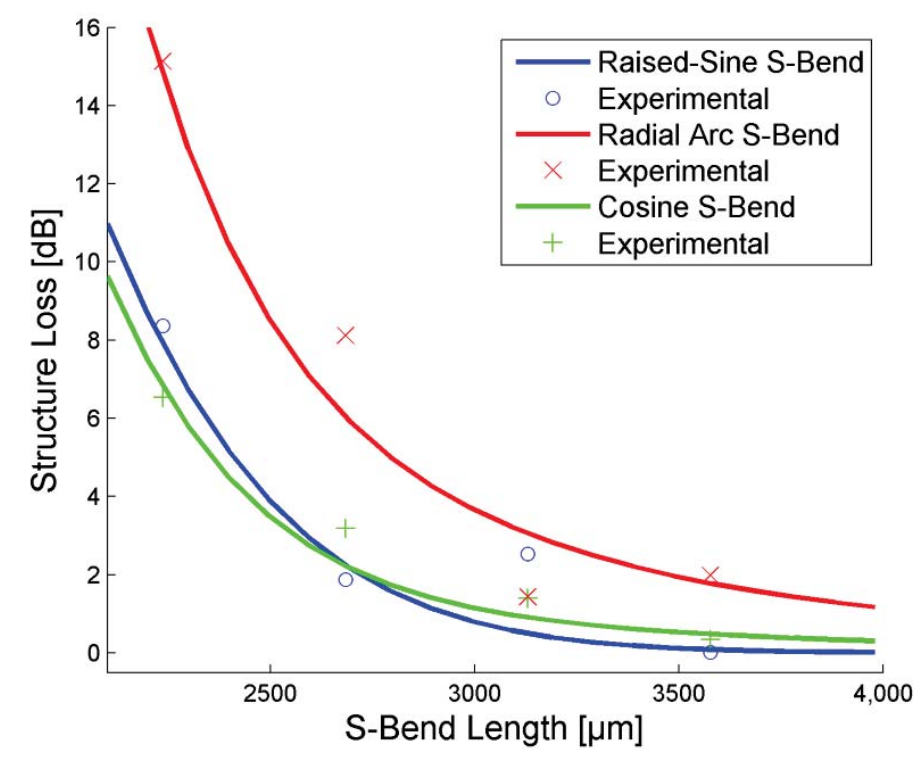

Figure 5.17 Structure loss of polymer SM WG S-bends manufactured through laser direct writing $(\lambda=1310 \mathrm{~nm})$.

Raised-sine S-bends have no discontinuities in their curvature profile and exhibit purely radial loss. Cosine and radial arc S-bends demonstrate both radial and transitional loss. Theoretical curves discussed in [84] were fitted to the experimental data to evaluate sources of loss. The radial loss coefficients $\left(C_{1}=0.053\right.$ [unitless], $\left.C_{2}=1.85 \mathrm{~mm}^{-1}\right)$ remained fairly constant between LDW and lithographic WG builds. In contrast the 
transitional loss coefficient for LDW polymer WGs $\left(\mathrm{T}_{1}=6.3 \mathrm{~mm}^{2}\right)$ was significantly higher in contrast to lithographic builds $\left(\mathrm{T}_{1}=1.6 \mathrm{~mm}^{2}\right)$. Radial arc S-bends suffer severe levels of structure loss due to high transitional loss present at its junction of the two mirrored radial bends.

Polymer WGs with a graded-index refractive index profile would result in increased transitional loss. Graded-index WGs allow larger lateral shifts of radial mode profile within the WG region without additional lengthening into the caustic region leaving radial loss indifferent $[85,86]$. Similar effects were observed when photolithographic and LDW SM WG radial bend experimental data was compared and correlated with theoretical plots, shown in Figure 5.18 [87].

\subsubsection{S-Bend Foot-print Requirements}

The structure loss of SM WG S-bends of constant width is strongly dependent on device length. For S-bends of various widths equivalent structure loss is obtained when a quadratic ratio between S-bend length and width is maintained, as described by Eq. (5.4). This demonstrates declining length requirements for equivalent loss when S-bend structures with larger widths are required. This relationship in S-bend builds has been observed by previous authors $[31,88]$. 


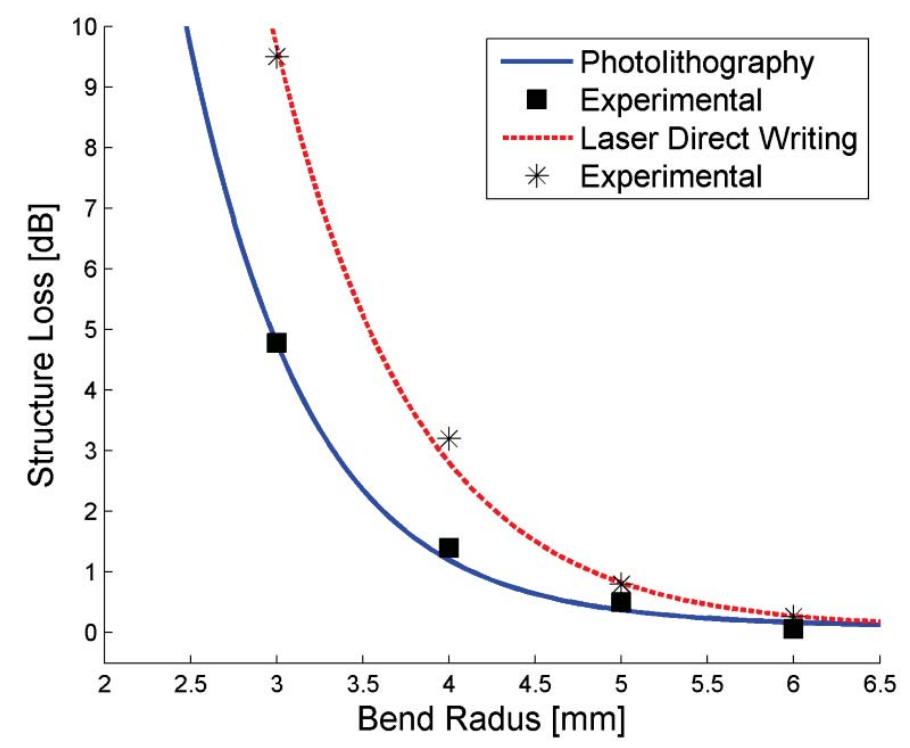

Figure 5.18 Theoretical (line) and experimental (marker) structure loss of polymer WG radial bends fabricated through photolithography and laser direct writing.

$$
\text { Loss } \propto \frac{L}{\sqrt{W}}
$$

Both structure loss and propagation loss need to be considered to obtain optimal device parameters for minimum total loss. While structure loss exponentially decreases with longer S-bend lengths, propagation loss linearly increases with longer S-Bend lengths, as shown in Figure 5.19. The summation of these loss factors as a function of S-Bend length demonstrates optimal S-bend dimensions required for minimum loss. 


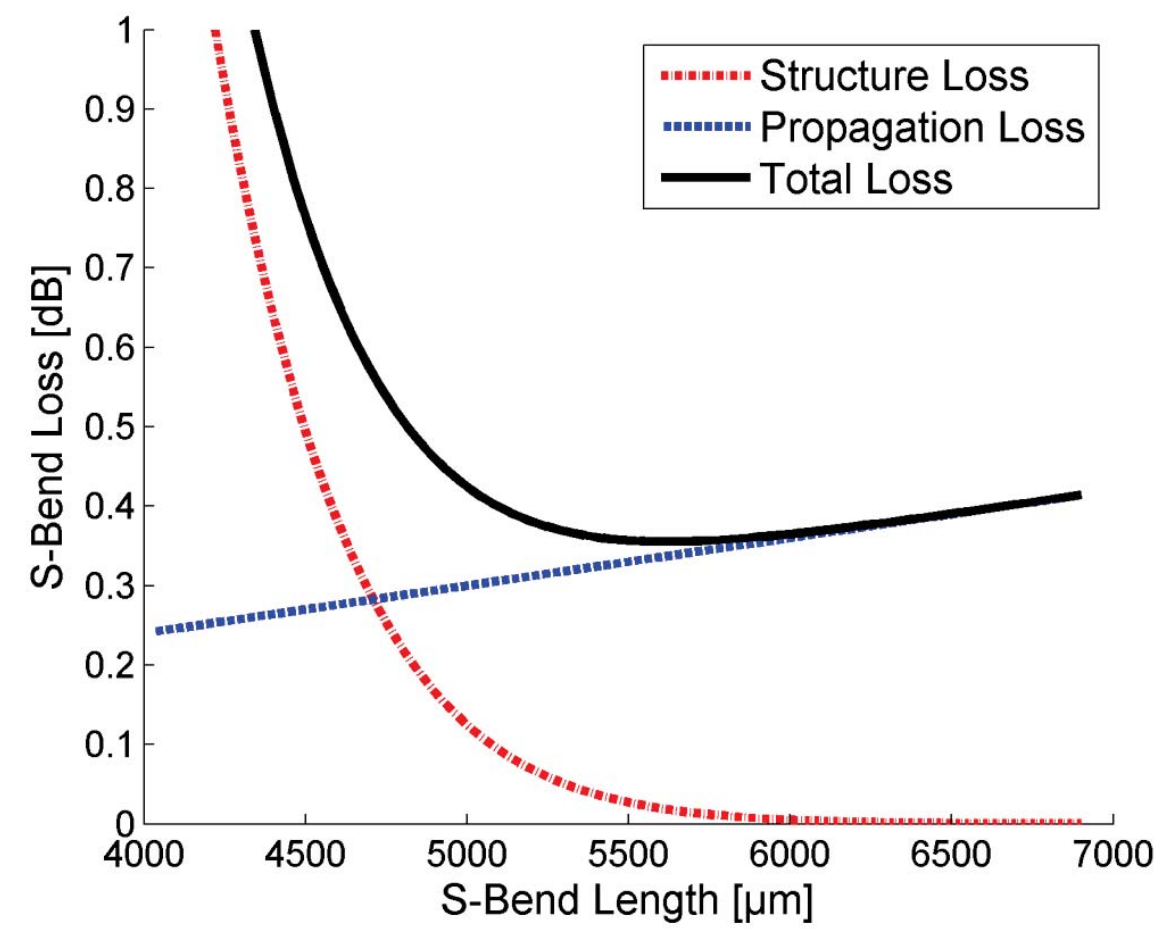

Figure 5.19 Structure, propagation, and total loss of raised-sine S-bends $[W=1000 \mu \mathrm{m}]$.

Raised-sine S-bends are preferred as the S-bend design of choice when WGs exhibit low propagation loss $(<1 \mathrm{~dB} / \mathrm{cm})$. If device footprint requirements overshadow minimal device loss, cosine and radial arc S-bends are preferred for low and high NA WGs respectively due to their lower structure loss at shorter S-bend lengths. This is important when using WG material with high absorption loss $(>1 \mathrm{~dB} / \mathrm{cm})$ where both propagation loss and structure loss need to be balanced for optimal device performance. If higher design efficiency is required, exotic S-bend structures, including WG offsets [46, 89] and air trenches [90], can be implemented. 
S-bend-based WG splitters require adiabatic tapers to reduce loss and minimize size requirements for efficient splitting. Even with the inclusion of adiabatic tapers overall optical splitting loss increases as the device size is decreased. Multimode interference devices could also be utilized with a smaller footprint [91], but their wavelengthdependence and environmental sensitivity must be taken into consideration for various Sbend applications.

\subsection{SM WG S-Bend Conclusion}

SM WG S-bends are important structures for realizing minimum optical loss and maximizing optical interconnect density for many applications in optical-integrated devices including directional control, pitch adjustment, and optical splitting. S-bend structures are thoroughly analyzed to realize optimal S-bend design for WGs of various NA and dimensions. Raised-sine S-bends exhibit the shortest S-bend length requirements necessary for minimum structure loss $(<0.1 \mathrm{~dB})$. For builds with smaller footprint requirements, cosine and radial arc S-bends are the most efficient designs for low NA and high NA WGs respectively.

$6 \mu \mathrm{m}$ SM WG S-bends were fabricated through the photolithographic process and optical measurements were correlated to theoretical models to obtain radial and transitional loss coefficients. Optical splitters fabricated with $1 \mathrm{~dB}$ and $3 \mathrm{~dB}$ S-bends exhibited splitting ratios of $49: 51 \pm 2 \%$ and minimal splitting losses were observed when S-bend splitters were fabricated with adiabatic tapers. 
LDW polymer WG S-bend designs were also evaluated utilizing the segmentation method for efficient implementation of complex WG writing using G-code. Optimal segmentation length $\left(L_{s}=10-50 \mu \mathrm{m}\right)$ exhibited the lowest device loss for LDW S-bend builds $(25 \mathrm{~mm} / \mathrm{s})$. Segmentation lengths outside optimal values exhibit high loss due to high angle bends and platform translational noise. Segmented (G1, $\left.L_{s}=20 \mu \mathrm{m}\right)$ and smooth (G2, G3) radial arc S-bends exhibited negligible differences in structure loss. Radial arc, cosine, and raised-sine S-bends were fabricated through segmentation $\left(L_{s}=20\right.$ $\mu \mathrm{m})$ and exhibited high transitional loss in comparison to photolithographic builds through theoretically evaluation.

SM WG S-bends showed equivalent loss when a quadratic relationship between S-bend width and length was maintained. Cosine S-bends were shown to exhibit the lowest structure loss for both photolithographic and LDW polymer WG builds. Experimental progress has shown that the LDW method is a suitable to replacement for traditional photolithography in the cost-efficient manufacturing of large-scale polymer WGs. 


\section{Chapter 6: Polymer WG Tapers}

Polymer waveguide taper designs are discussed as connectorization devices for improving coupling efficiency between silicon WGs and SMFs. The functionality of tapered polymer WGs as mode conversion devices is theoretically discussed. Vertical and bilayer taper designs are proposed for cost-efficient optical coupling and compared taking into consideration both design requirements and manufacturing feasibility. Optimal device dimensions and alignment tolerances for both designs are determined through optical simulation to determine peak coupling efficiency with both Si rib and Si wire WGs.

SM polymer WG S-bends and tapers can be integrated into polymer WG bridges providing low loss fan-out techniques and coupling between on-chip transceiver devices containing Si waveguide structures to traditional single mode optical fiber. Bridge module structure requirements are reported for a 12 member polymer WG bridge module utilizing fabrication results from polymer S-bends and tapers.

\subsection{Introduction}

Silicon (Si) waveguides (WGs) are used as high bandwidth optical communication channels $(\lambda=1300-1600 \mathrm{~nm})$ within integrated devices and are readily fabricated using traditional semiconductor manufacturing techniques $[92,93]$. The high refractive index of silicon $(n=3.5)$ ensures strong confinement of light within the waveguide that is 
typically a few hundred nanometers in size. Optical interconnects to transfer light into and out of integrated circuits are required to maximize data transmission rates on-board and off-board applications. Direct connection can be performed using silica-based single mode fibers (SMF) that possess low attenuation rates $(0.2 \mathrm{~dB} / \mathrm{km})$ and allow for flexible connectivity [37]. Polymer WGs with relatively low attenuation rates $(0.05 \mathrm{~dB} / \mathrm{m})$ are also utilized as off-chip transmission lines for their cost-effective fabrication and integration in printed wiring boards $[38,39]$.

Direct coupling between Si WGs $(\mathrm{NA}>3.0)$ and SMFs $(\mathrm{NA}<0.15)$ results in high coupling loss $\left(\Gamma_{C}=18.8 \mathrm{~dB}\right)$ [94], which arises from the modal size and numerical aperture (NA) mismatch between the Si WGs (NA > 3.0) and SM dielectric WGs (NA < 0.15). Modal mismatch between Si WGs and SMFs can be observed through the mode profiles as shown in Figure 6.1. The narrow cone of acceptance $\left(\sim 8^{\circ}\right)$ for SMFs means that only a very small portion of the output power of the Si WG, resulting in high coupling losses. In a bidirectional approach, Si WGs $(\sim 300 \mathrm{~nm})$ cover only a fraction of the SMF core $(\sim 8 \mu \mathrm{m})$ and limit the effective coupling from SMFs. Mode conversion devices are required to minimize coupling efficiencies between high density Si WG devices and off-chip communication channels.

Silicon-based tapers have been previously proposed as modal expansion devices for reducing coupling losses with external devices [95]. Tapers expand and delocalize the propagating mode by adiabatically tapering down the core [94]. Multilayer tapers use a two-layer system to expand the WG and its fundamental mode size before exiting the $\mathrm{Si}$ 
WGs [33]. While the multilayer tapers theoretical coupling losses are low (0.5 dB) [34] they require careful procedures to accurately shape, align, and cleave the tapered ends of the silicon photonic chips.
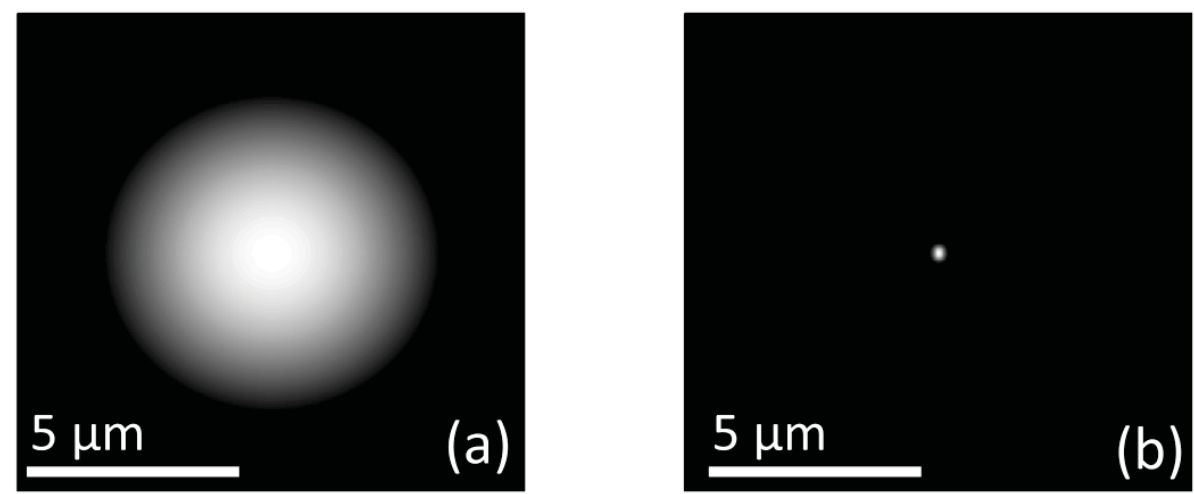

Figure 6.1 Simulated mode profile $[\lambda=1310 \mathrm{~nm}]$ of $8 \mu \mathrm{m}$ diameter $\mathrm{SMF}(\mathrm{a})$ and $400 \mu \mathrm{m} \times 200 \mu \mathrm{m}$ Si $W G(b)$.

Previous research has sought to improve coupling efficiencies by shaping the ends of SMFs. Tapered [36] and lensed [35] SMFs are utilized to reduce optical coupling losses, reducing the output beam spot size of the SMFs. Both designs require complex and expensive procedures to individually fabricate and align the optical fibers for coupling with Si WG arrays [96].

Modifications of the Si WG or the SMF end-face require costly manufacturing procedures to accurately cut and clean the optical interconnect end-faces. It is beneficial to design a mode-coupling module which can be implemented between flat end-faced silicon photonic WGs and SMFs. A photonics-bridge module which minimizes the 
optical coupling losses allows for system flexibility in device connectorization while eliminating the expenses required for conducting end-face treatments on both photonic WGs and devices.

A photonics-bridge module utilizing a novel polymer WG taper design, shown in Figure 6.2, is implemented to overcome the direct coupling limitations between Si WG and SMF. Polymer WG materials are ideal materials for mode-conversion devices as they exhibit low absorption losses and can be molded through a variety of cost-effective manufacturing techniques including photolithography [97] and soft-imprint lithography [98]. WG taper designs are proposed for planar coupling between Si WGs and SMFs and evaluated in terms of coupling losses, length requirements, and alignment tolerances. Bridge modules would minimize optical coupling loss, allow for system flexibility in device connectorization, and eliminate the expenses required to attach NA-matching devices onto optical interconnect end-faces.

Overall module design is also outlined with an emphasis on pitch control, bending loss, and cross-talk analysis. Experimental results on SM polymer WG straights, S-bends, and tapers were utilized to determine both device performance and minimum size requirements for low-loss bridge modules between high-density Si WG photonic interconnects and long-haul SMFs. 


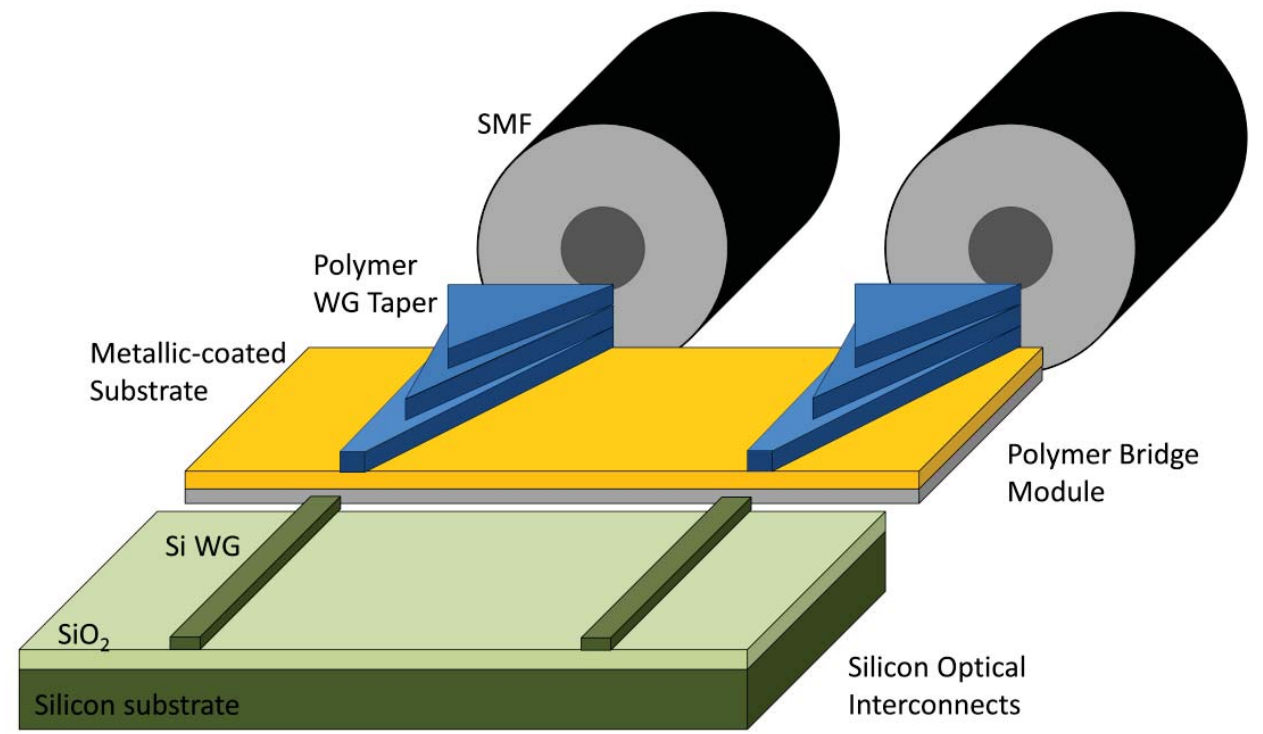

Figure 6.2 Polymer taper bridge module illustration.

\subsection{Polymer WG Taper Theory and Designs}

\subsubsection{Taper Theory}

The coupling efficiency, $\Gamma_{C}=\Gamma_{T}$, between two optical waveguiding structures can be calculated using an overlap integral [49] between the two mode profiles, previously described by Eq. (2.11). Waveguide tapers implement a simplistic mode-transformer to improve modal overlap with the WG devices by slowly transforming the fundamental mode as it propagates through the device. The modal profile of the Si WG significantly overlaps with the polymer WG taper tip mode profile and results in high coupling efficiency when the taper tip's dimensions are comparable to those of Si WGs. While 
acting as a mode conversion device polymer WG tapers (NA 1.1) still exhibit coupling loss at both end-faces with Si WGs (NA 3.1) and SMFs (NA 0.1).

The polymer WG taper's capability to efficiency condense optical power to acquire significant overlap with Si WGs is determined by its NA. Tapers with a high NA $(\geq 1.0)$ are required to acquire strong $(<1 \mu \mathrm{m})$ modal confinement at the taper tip for effective overlap with the highly divergent, concentrated mode output of Si WGs, as shown in Figure 6.3. A high NA $(>1.0)$ is required to obtain tight $(<1 \mu \mathrm{m})$ modal confinement within polymer WG tapers. The tapered region is surrounded by reflective metals or low dielectric materials to maintain a high NA and ensure modal confinement at sub-micron dimensions throughout the taper.

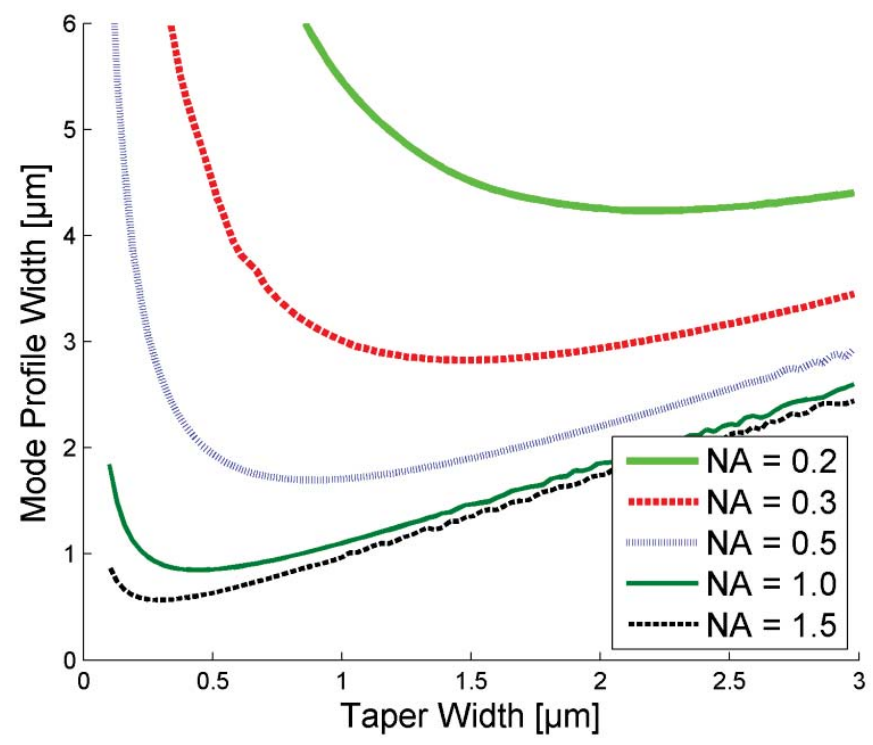

Figure 6.3 Simulated mode width in polymer WGs as a function of NA and tapered $W G$ width $(\lambda=1310 \mathrm{~nm})$. 
High NA polymer tapers tips with dimensions larger than the SM cutoff $(0.8 \mu \mathrm{m}$ for symmetric WGs, NA $=1.1, \lambda=1310 \mathrm{~nm}$ ) permit increasing levels of the excitation and propagation of higher-order modes within the taper. A wavelength of $1310 \mathrm{~nm}$ was utilized to minimize absorption loss in both Si WGs and polymer WGs. Higher mode structures experience modal interference resulting in modal dispersion. Each excited mode propagates at a different velocity inducing in a fluctuating E-field that is a function of taper length. The significant differences in E-field fluctuations between single mode and multi-mode coupling within polymer tapers are shown in Figure 6.4. The selfimaging effects of multi-mode interference create a varying output profile at the output of the tapered WG resulting in large deviations in device coupling if the length of the taper is not significantly controlled [42].
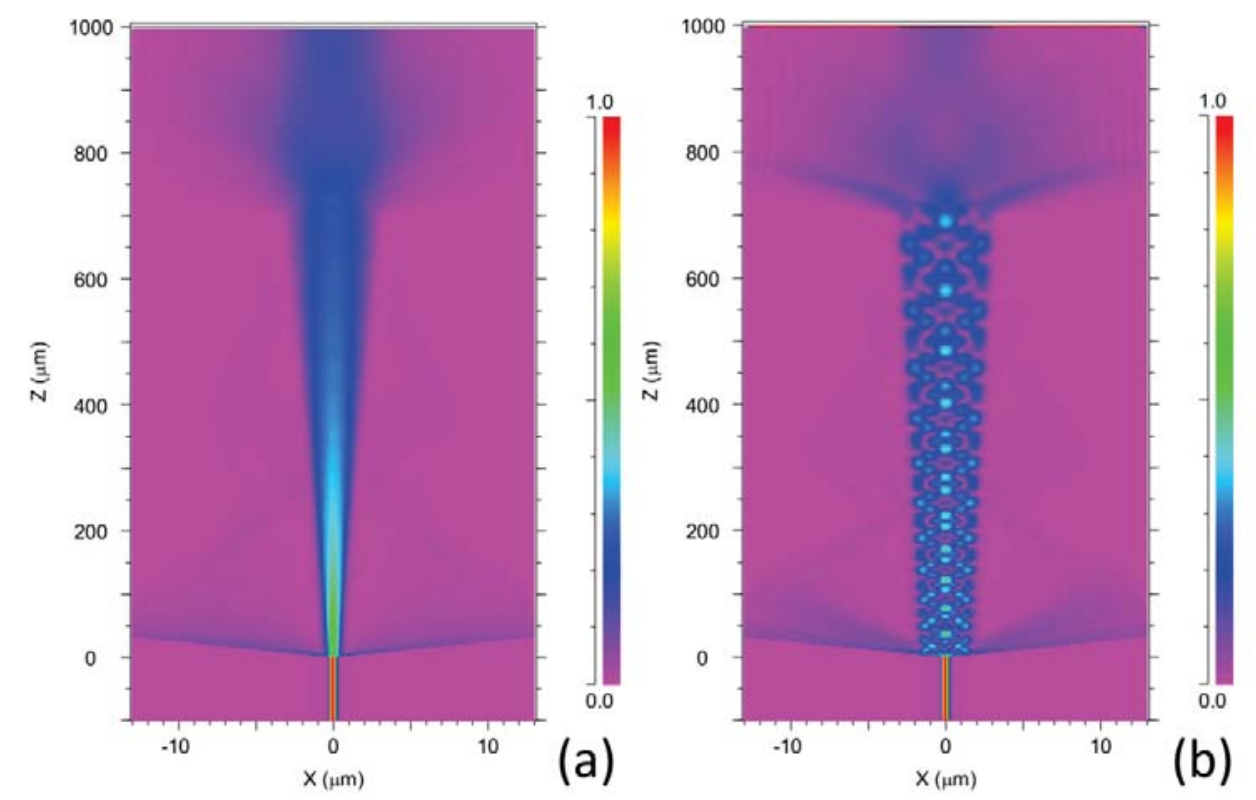

Figure 6.4 Si WG modal excitation in polymer WG tapers with a $0.5 \mu \mathrm{m}$ (a) and $3.0 \mu \mathrm{m}$ (b) WG taper width. 


\subsubsection{Taper Design}

Practical polymer WG tapers out in three dimensions while being immersed in a suitable medium for internal reflectivity. While most polymer waveguides can be formed through the simple procedures of traditional photolithography, underside vertically-tapered polymer WGs require complex fabrication procedures with the assistance of temporary or permanent resists for molding the vertical tapers. WG tapers should be designed for commercial feasibility with minimal fabrication costs. Two alternative polymer WG taper designs with a reflective underside have been proposed to overcome the complications associated with the fabrication of an underside taper.

Polymer WG tapers can be molded with a vertical taper, shown in Figure 6.5, while maintaining contact with the reflective substrate. The design tapers horizontally and vertically between WG end-faces with specified dimensions optimal for signal coupling. Horizontal tapers can be processed lithographically and then vertically shaped to form the vertical taper. Vertical tapers can also be formed through polymer molding [98] or grayscale etching [97]. WG taper coupling loss will depend on the final device's surface roughness, minimizing higher-order mode excitation.

The multi-layer taper, an alternate design shown in Figure 6.6, allows for mode vertical modal expansion without the requirement of vertical tapering. The bottom taper starts at a specified width and tapers out horizontally maintaining a constant height. Additional tapers are fabricated on top and the total device height is set to match the polymer WG height. Utilizing an optimal taper tip the coupled mode adiabatically expands into the top 
tapers as the device expands horizontally. In contrast to vertical tapers multi-layer tapers can be manufactured through traditional, $0.5 \mu \mathrm{m}$ resolution multi-step photolithography.

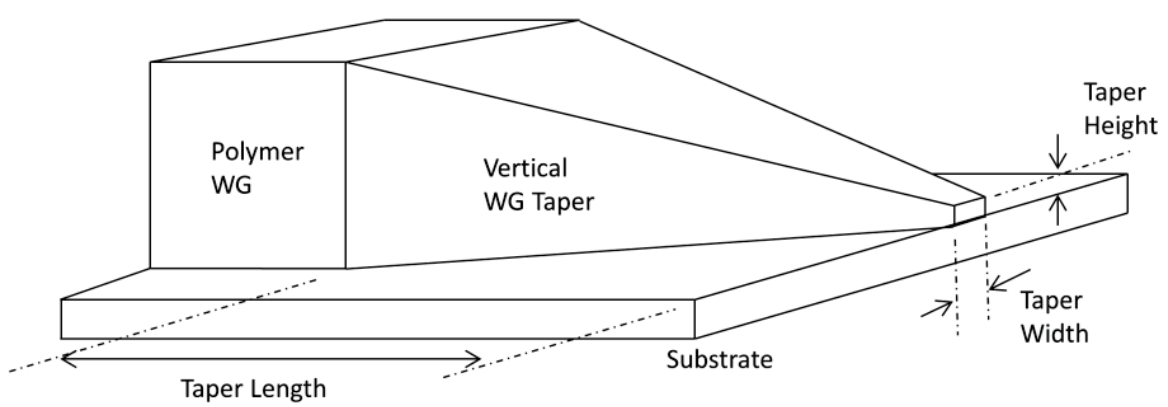

Figure 6.5 Vertical taper illustration

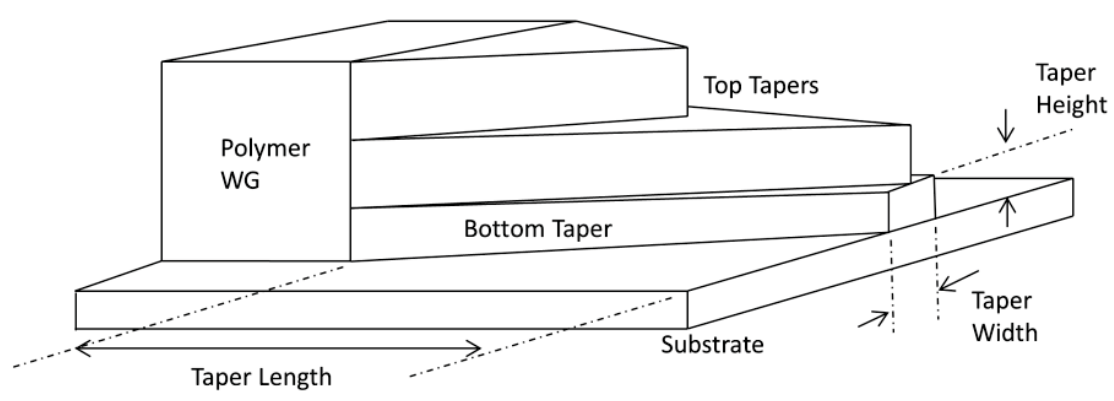

Figure 6.6 Multi-layer taper illustration.

Both vertical and multi-layer taper designs require an appropriate lower cladding to ensure a high NA and prevent modal leakage into the substrate $[99,100]$. The refractive index of minimal dielectric materials is limited to $\mathrm{n} \geq 1.29$ (NA $\sim 0.78$ ) restricting its suitability for Si WG coupling [101]. A highly reflective metallic coating is a costefficient alternative for a substrate but will cause a polarization-dependent coupling loss 
due to its conductivity. The taper may alternatively be undercoated with a dielectric Bragg mirror for polarization-invariant mode conversion devices [102] but design and functionality will be dependent on wavelength and taper length to ensure modal confinement during adiabatic mode transformation [103]. The rest of the polymer WG taper can be left uncoated since an air or vacuum environment allows for a high NA $(\sim 1.1)$ while reducing fabrication costs.

\subsection{WG Taper Simulation Results}

Mode conversion design performance was simulated using the Beam Propagation Method (BPM). 3D BPM results were used to correlate taper dimensions and misalignment tolerances with device coupling efficiencies. The refractive index of the polymer taper was set to match that of Dow Corning's UV-curable optical elastomer ( $\left.n_{\text {core }}=1.5142\right)$ [87]. The taper region was surrounded by air $\left(n_{\text {clad }}=1\right)$ to demonstrate NA matching between Si WGs and SMFs. A wavelength of $1310 \mathrm{~nm}$ (TE polarization) was used to simulate optical coupling to minimize system absorption loss for both Si WGs and polymer WGs. Boundary reflections at the polymer taper interfaces are not taken into account in BPM simulations but can be integrated using a multiplicative factor based on various integration methods. 


\subsubsection{Taper Length Requirements}

Polymer WG taper devices require a minimum length to realize adiabatic mode conversion where the coupled mode is expanded without excitation to radiation, leaky, or higher-order modes. This overall dependence between coupling loss and taper length for vertical and multi-layer polymer tapers is shown in Figure 6.7 and Figure 6.8 respectively. BPM simulation results revealed that adiabatic length requirements are dependent on taper design but independent of taper tip dimensions.

The adiabatic lengths for vertical tapers and multi-layer tapers are $200 \mu \mathrm{m}$ and $600 \mu \mathrm{m}$ respectively. The length requirement in multi-layer taper is significantly longer due to the modal transformation between multiple stacked tapers. Shorter multi-layer taper lengths result in the excitation of vertical higher-order modes from self-imaging resulting in increasing variations in taper loss as the taper length is decreased. The multi-layer taper design was observed to require at least three layers for efficient mode conversion. Negligible improvements in coupling efficiency were observed if more $(>3)$ layers were incorporated. The coupling loss for polymer WG tapers is independent of the length of the waveguide taper beyond the limit for adiabatic expansion [96].

Coupling symmetry is also important in bridge module designs. Device loss when coupling from Si WGs to SMF (mode expansion) should be identical to cases for coupling from SMFs to Si WGs (mode condensing). BPM simulations show that polymer tapers exhibit minimal $(<0.2 \mathrm{~dB})$ variations in taper loss at adiabatic taper lengths, as demonstrated in Figure 6.9. 


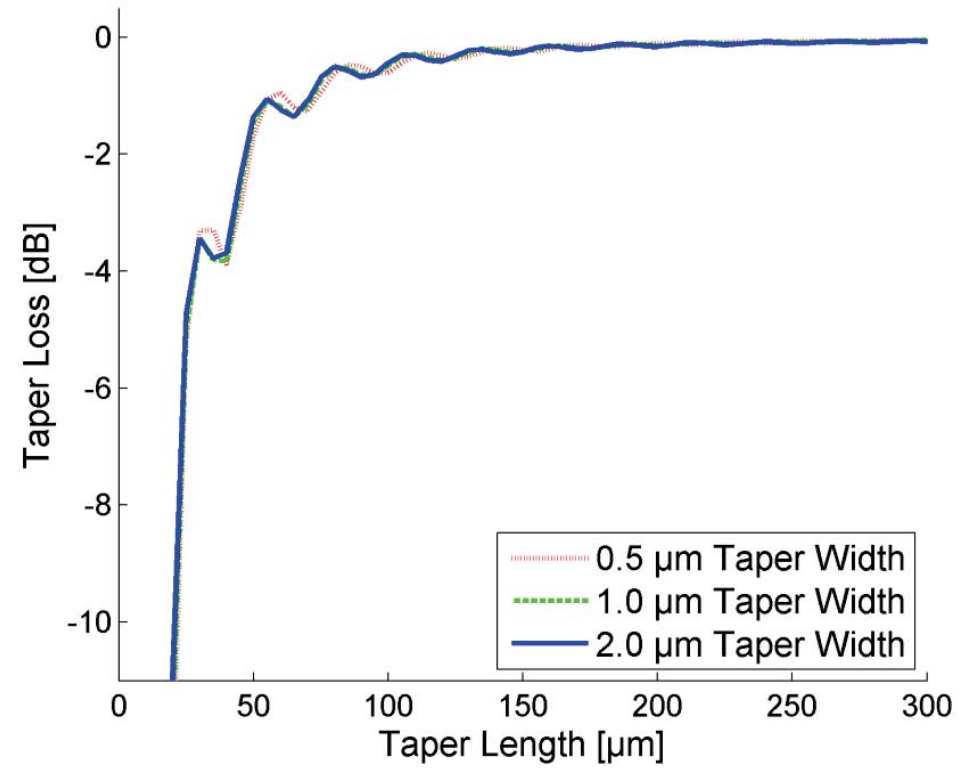

Figure 6.7 Taper loss as a function of taper length for vertical tapers $(0.5 \mu \mathrm{m}$ Taper Height, $\lambda=1310 \mathrm{~nm}$ ).

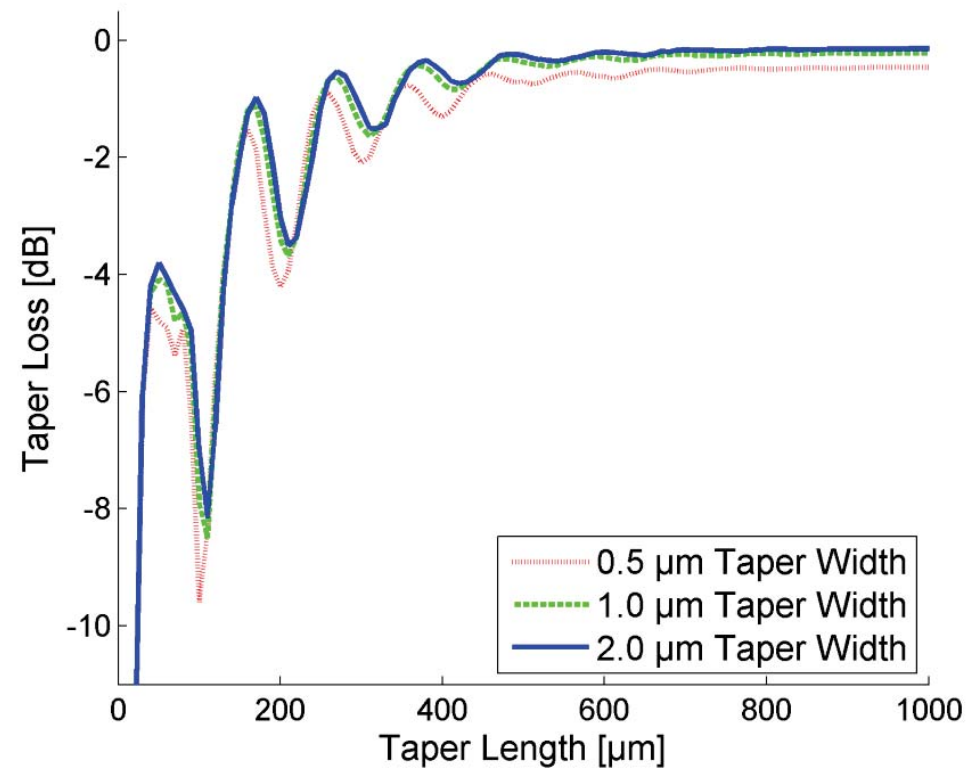

Figure 6.8 Taper loss as a function of taper length for multi-layer tapers $(0.5 \mu \mathrm{m}$ Taper Height, $\lambda=1310 \mathrm{~nm}$ ). 


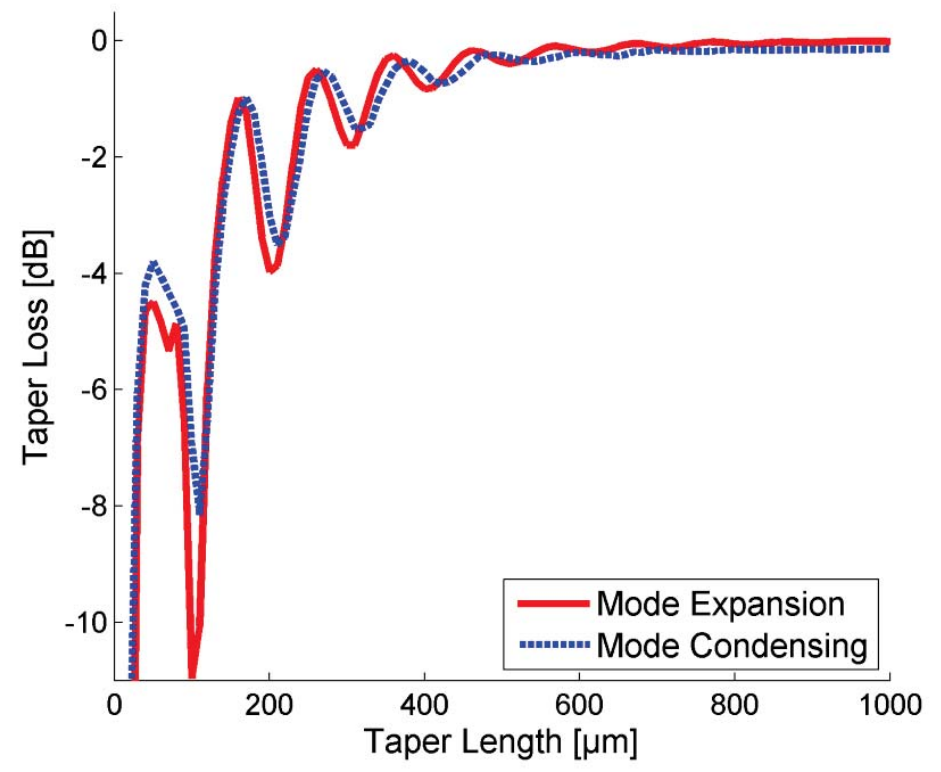

Figure 6.9 Directional taper loss in multi-layer tapers as a function of taper length. (0.5 $\mu \mathrm{m}$ Taper Width, $0.5 \mu \mathrm{m}$ Taper Height, $\lambda=1310 \mathrm{~nm})$.

Mode conversion efficiency in multi-layer taper is also dependent on the fabrication resolution required to minimize the end widths of the top tapers. Lower resolutions result in tapers with reduced coupling efficiency between layers by exciting both higher-order modes and radiation modes [104]. Minimal taper widths exist for overlaying tapers to minimize taper loss, as shown in Figure 6.10 [33]. Resolution requirements for multilayer tapers decrease with wider underlying layers.

Multi-layer tapers with a $2 \mu \mathrm{m}$ taper width exhibit $1 \mathrm{~dB}$ taper loss when the tip width of the overlying tapers is $700 \mathrm{~nm}$ respectively, as shown in Figure 6.10. These tolerances are more relaxed in comparison to the $200 \mathrm{~nm}$ width tolerances required for Silicon WG 
Taper designs [33, 34]. Relaxed resolution requirements allow for the multi-layer taper design with large taper widths to be fabricated with multi-step traditional photolithography using UV-curable materials $[105,106]$.

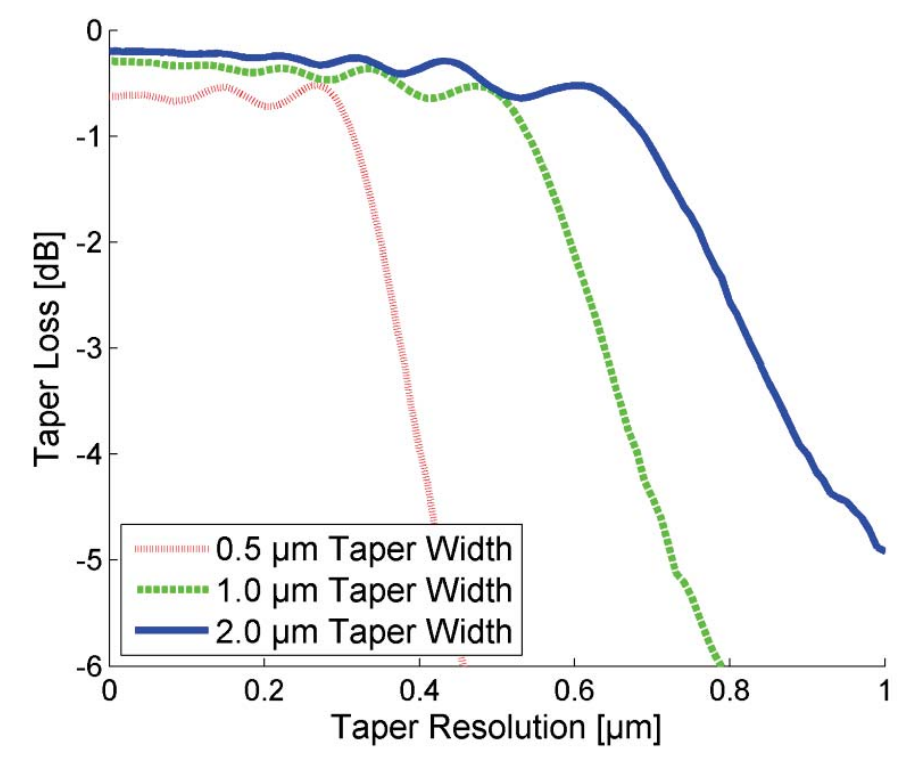

Figure 6.10 Multi-layer taper loss as a function of lithographic resolution $(\lambda=1310 \mathrm{~nm})$.

\subsubsection{Coupling Loss}

The taper tip dimensions are crucial in controlling the device's ability to maximize coupling efficiency with Si WGs. The dimensions required for optimal coupling is dependent on the type and size of the Si WG and its resulting mode profile. SM mode profiles of both Si wire WGs (200 nm x $350 \mathrm{~nm})$ and Si rib WGs (200 nm x $1000 \mathrm{~nm}$, $25 \mathrm{~nm}$ rib height) were used as launch fields to demonstrate mode converter's 
compatibility with both Si-based photonic WG structures, shown in Figure 6.11. Polymer tapers maximized to $6 \mu \mathrm{m} \times 6 \mu \mathrm{m}$ single mode WGs to maximize coupling efficiency with SMFs [84]. The coupling efficiency of WG tapers has been shown to be independent of waveguide taper length beyond the distance requirements for adiabatic mode conversion [96].
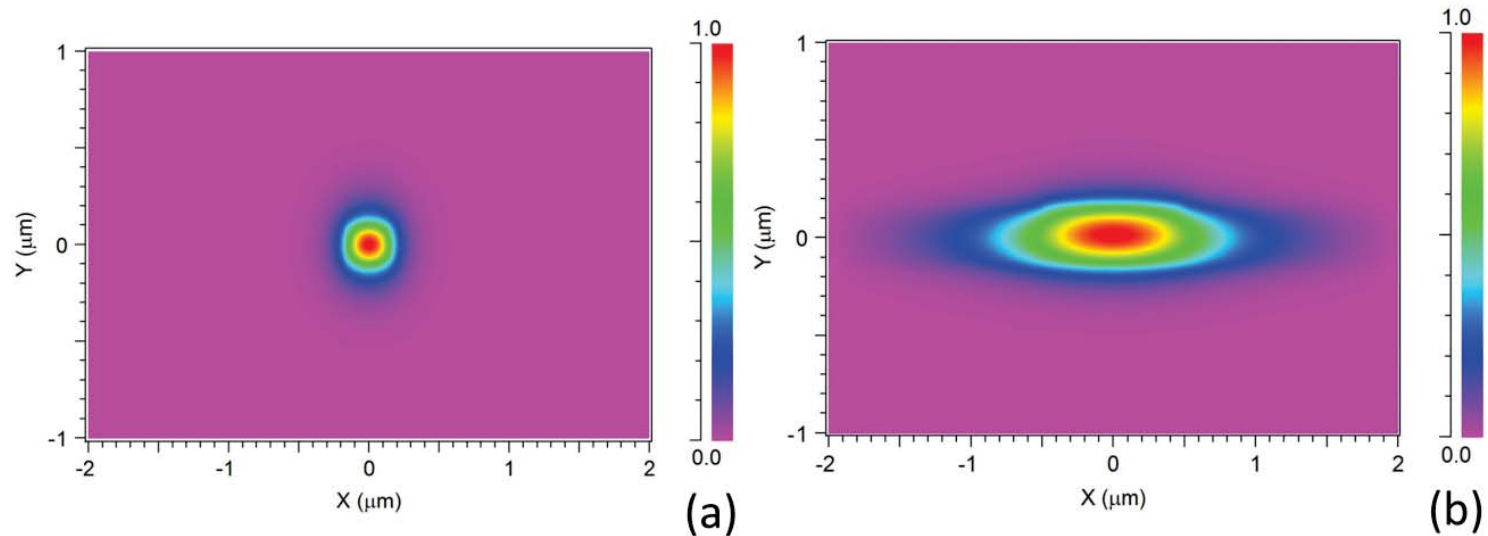

Figure 6.11 Mode profiles for SM Si Wire (a) and Si Rib (b) WGs ( $\lambda=1310 \mathrm{~nm})$.

The coupling efficiency between Si Wire WGs and SMFs utilizing polymer tapers is maximized with a $500 \mathrm{~nm}$ x $600 \mathrm{~nm}$ polymer WG taper tip, as shown in Figure 6.12. In contrast, a $2 \mu \mathrm{m}$ taper width maximized coupling efficiency with Si rib WGs, as shown in Figure 6.14. The larger mode profile of the Si rib WG $(2.75 \mathrm{~dB})$ exhibits both lower coupling loss in comparison to the Si wire WG $(4.25 \mathrm{~dB})$.

Bridge loss is a summation of both the coupling loss at the polymer taper end-faces and mode conversion loss within the taper. Eq. (2.11) indicates that both taper designs exhibit 
coupling loss $\left(\Gamma_{C}=1.10 \mathrm{~dB}\right)$ between the $6 \mu \mathrm{m}$ polymer WGs end and SMFs. Coupling theory also indicates optimal coupling loss for $\mathrm{Si} \operatorname{rib}\left(\Gamma_{C}=1.39 \mathrm{~dB}\right)$ and $\mathrm{Si}$ wire $\left(\Gamma_{C}=\right.$ 3.02 dB) WGs. The summation of coupling loss at both interfaces matches BPM simulation loss after omitting the slight difference in mode conversion efficiency previously demonstrated in Figure 6.7.

Coupling differences between taper designs were observed with larger taper widths. Expanding the dimensions of the taper tip increases the amount of higher-order modal excitation and raises the amount of self-imaging effects within the taper. This is observed as significant fluctuations in optical coupling as can be seen with wide $(>2 \mu \mathrm{m})$ taper widths. Multi-layer tapers are more sensitive to multi-mode coupling resulting in lower and more inconsistent coupling efficiencies with $2 \mu \mathrm{m}$ height tapers, as shown in Figure 6.13 and Figure 6.15. The stacked tapers may appear to the highly fluctuating E-field as abrupt shifts in WG height and result in high device loss.

\subsubsection{Misalignment Tolerances}

Alignment tolerances become a strong factor in module feasibility for future commercialization. Fabrication and implementation procedures require the WG taper designs to function either as external pick-and-place parts or devices manufactured directly on the Si photonic chip. 


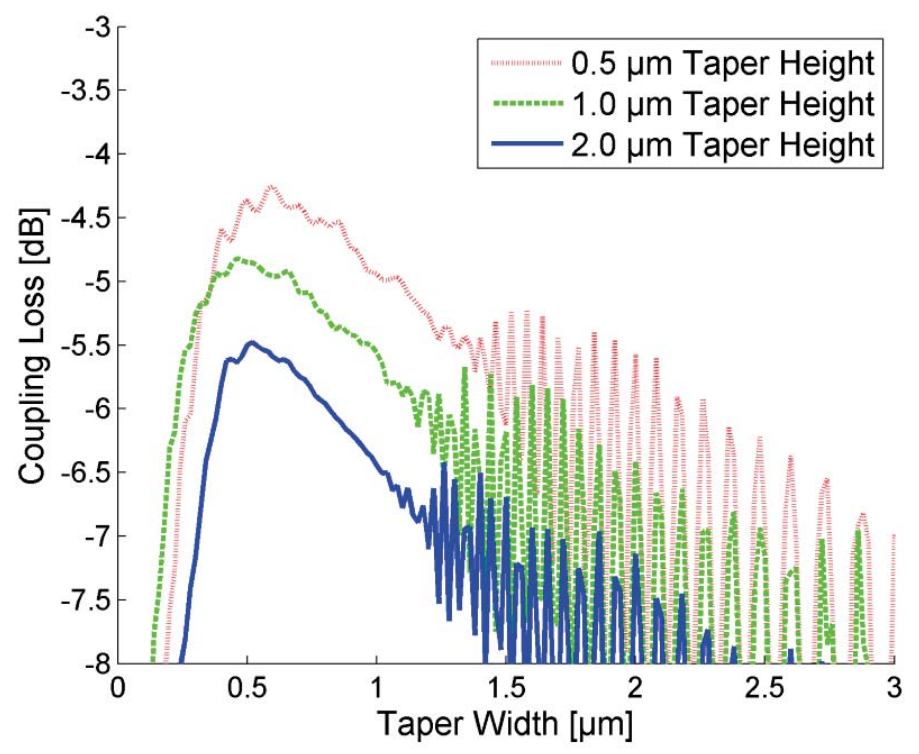

Figure 6.12 Coupling loss between Si wire WGs and SMFs utilizing vertical tapered WGs with various tip dimensions $(\lambda=1310 \mathrm{~nm})$.

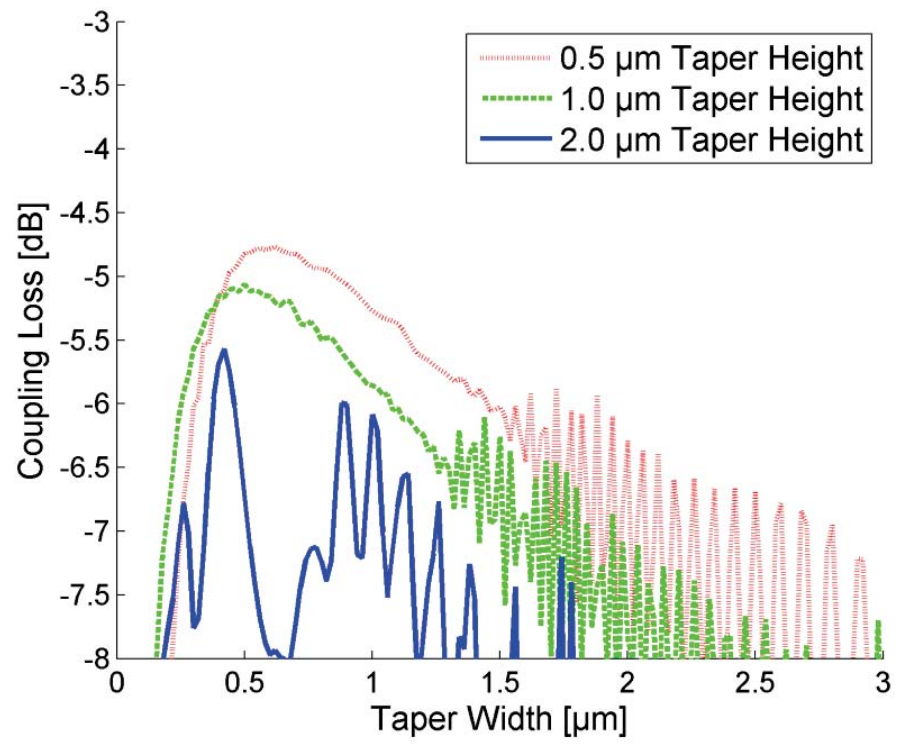

Figure 6.13 Coupling loss between Si wire WGs and SMFs utilizing multi-layer tapered WGs with various tip dimensions $(\lambda=1310 \mathrm{~nm})$. 


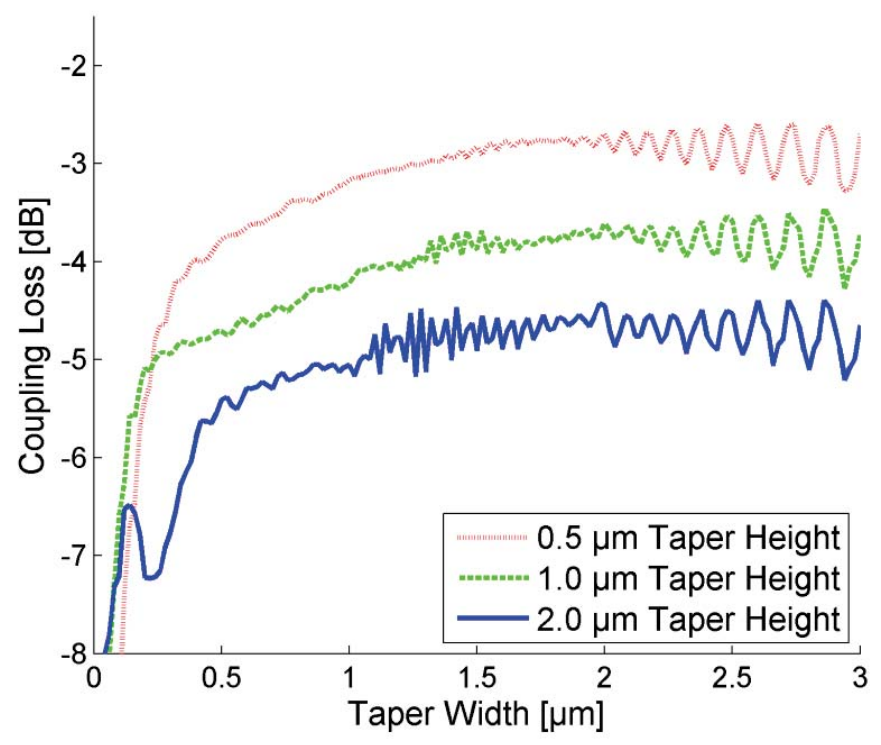

Figure 6.14 Coupling loss between Si rib WGs and SMFs utilizing vertical tapered WGs with various tip dimensions $(\lambda=1310 \mathrm{~nm})$.

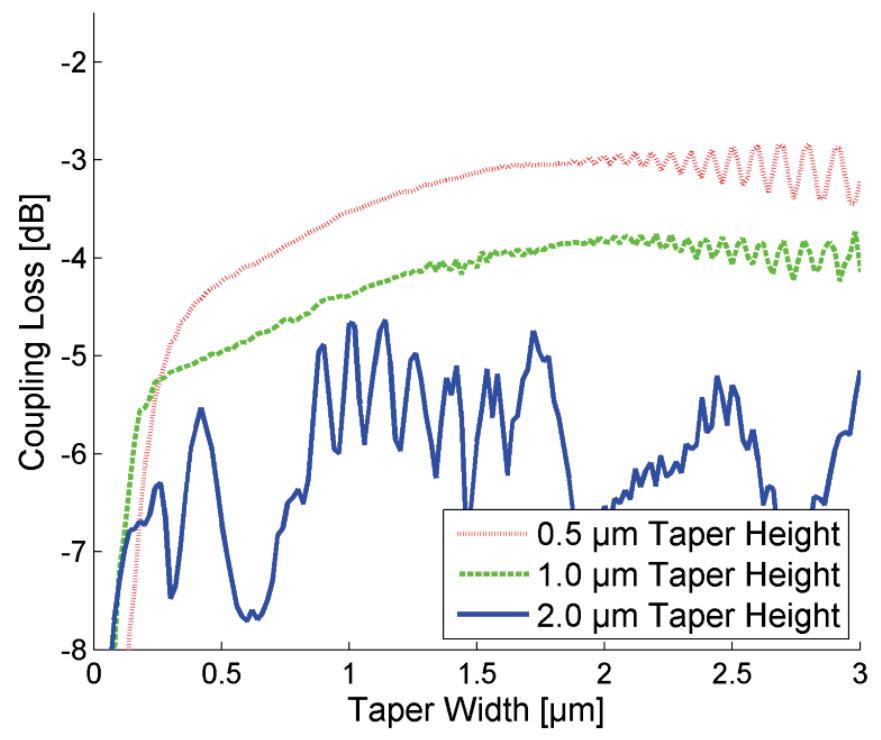

Figure 6.15 Coupling loss between Si rib WGs and SMFs utilizing multi-layer tapered WGs with various tip dimensions $(\lambda=1310 \mathrm{~nm})$. 
The normalized misalignment loss between polymer WG tapers and Si WGs is shown in Figure 6.16 and Figure 6.17. Increasing the taper width relaxes device alignment tolerances at the cost of reducing the maximum coupling efficiency. Polymer WG tapers with a $0.5 \mu \mathrm{m}$ taper width exhibit tight $3 \mathrm{~dB}$ misalignment tolerances $( \pm 0.3 \mu \mathrm{m})$ when coupled with Si wire WGs. Increasing the taper width to $1.5 \mu \mathrm{m}$ improves $3 \mathrm{~dB}$ misalignment tolerances $( \pm 0.4 \mu \mathrm{m})$ with $\mathrm{Si}$ wire WGs. Increasing the taper width from 2 $\mu \mathrm{m}$ to $4 \mu \mathrm{m}$ increases the $3 \mathrm{~dB}$ misalignment tolerances from $\pm 0.7 \mu \mathrm{m}$ to $\pm 1.2 \mu \mathrm{m}$ when coupling with Si rib WGs. Additional widening of the taper width comes with the drawback of decreased and inconsistent coupling efficiency due to multi-modal excitation.

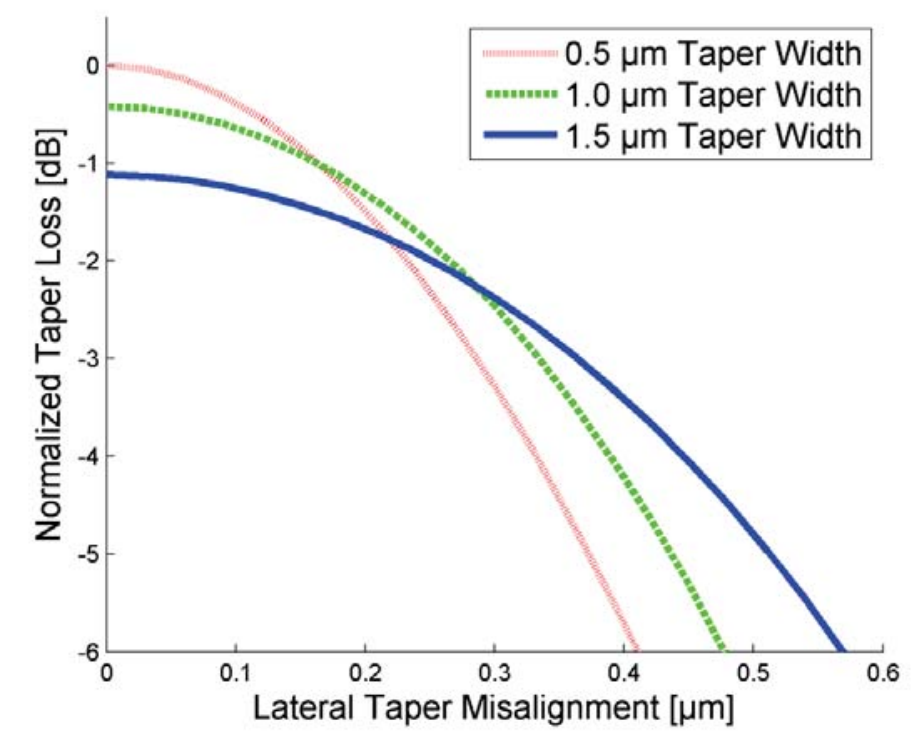

Figure 6.16 Lateral taper misalignment loss coupling with Si wire waveguides $(\lambda=1310 \mathrm{~nm})$. 


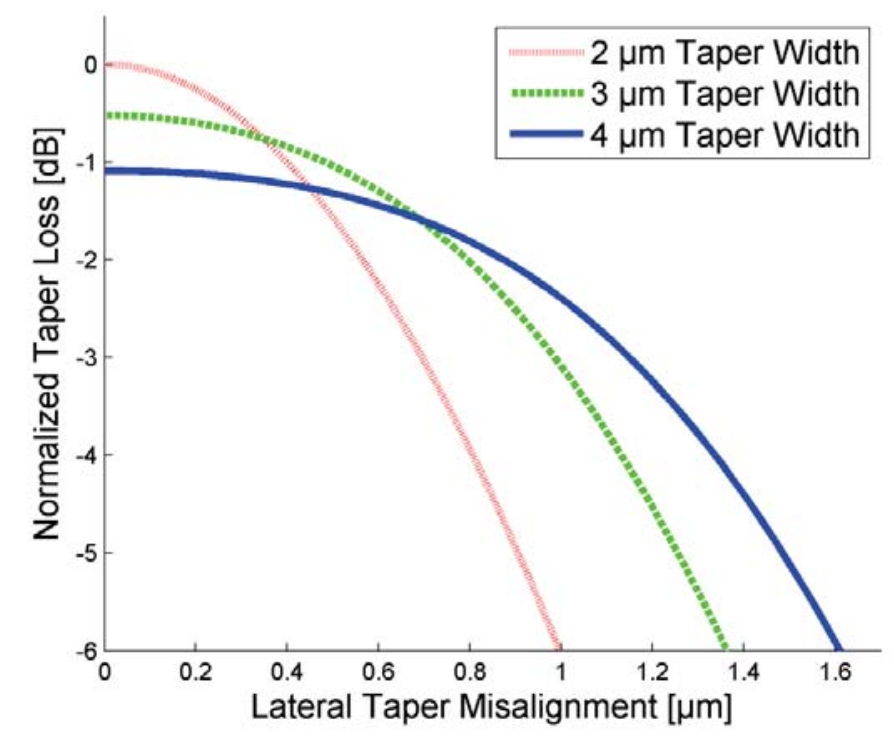

Figure 6.17 Lateral taper misalignment loss coupling with Si rib waveguides $(\lambda=1310 \mathrm{~nm})$.

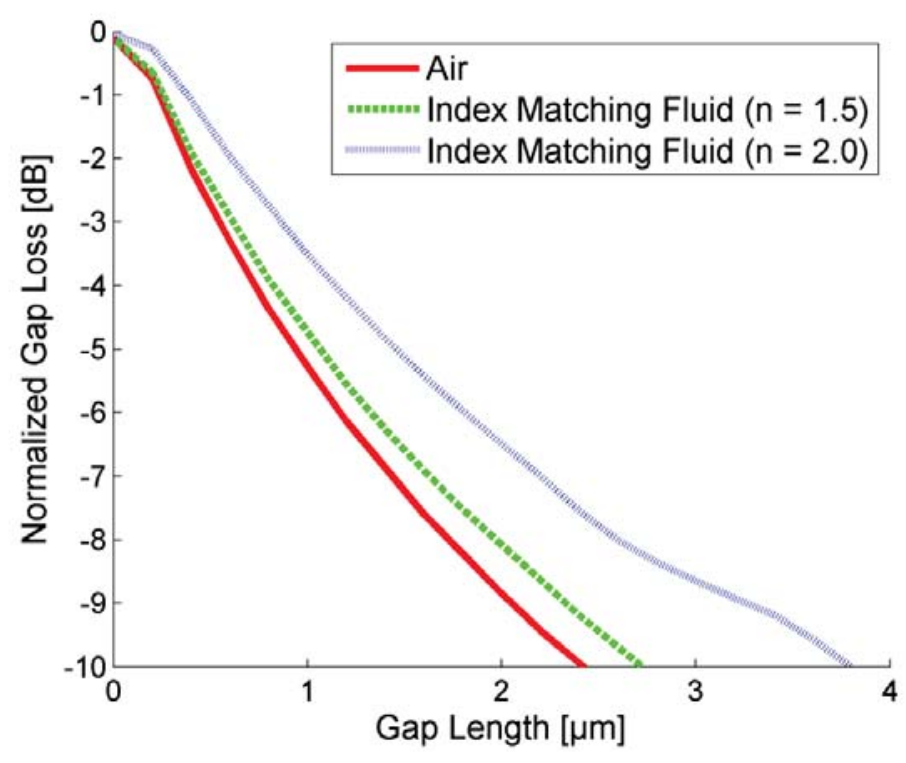

Figure 6.18 Gap loss when coupling with Si wire waveguides $(\lambda=1310 \mathrm{~nm})$. 


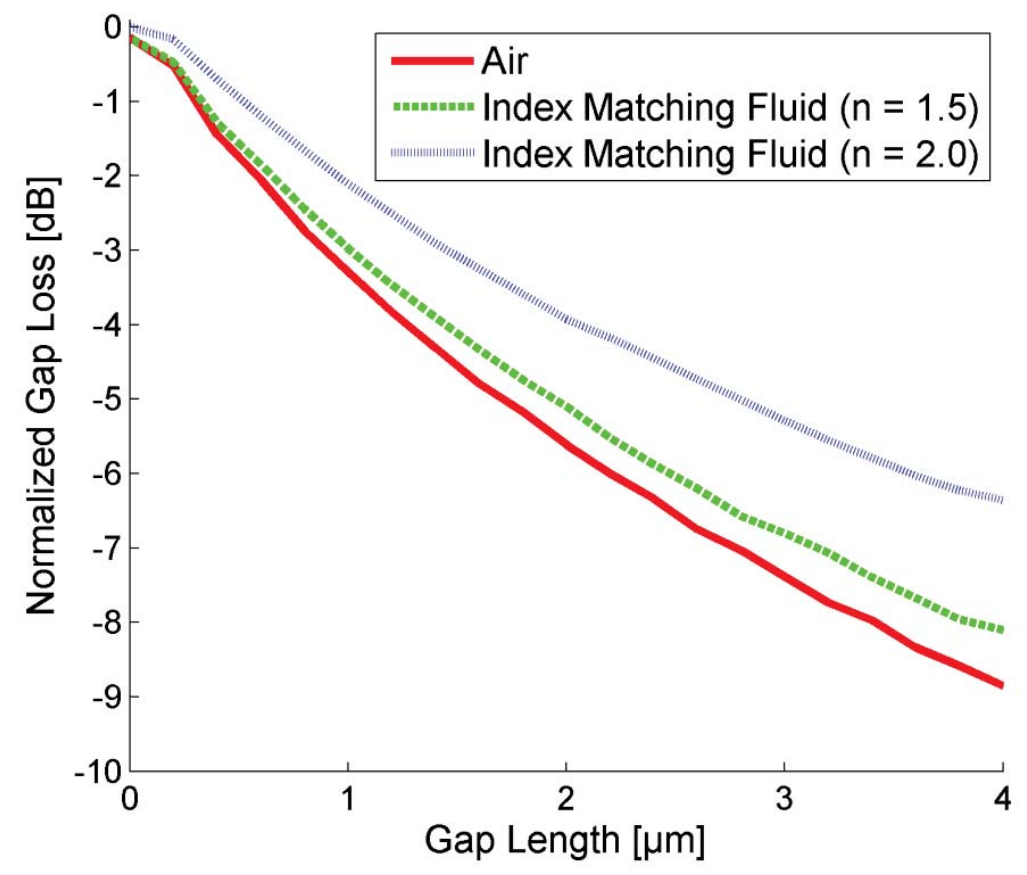

Figure 6.19 Gap loss when coupling with Si rib waveguides $(\lambda=1310 \mathrm{~nm})$.

If there is a gap present between the $\mathrm{Si} \mathrm{WG}$ and the polymer taper additional loss will be incurred. Gap loss is observed to decrease as the gap length increases, as shown in Figure 6.18 and Figure 6.19. Gap loss can be reduced if index matching fluid fills in the gap to reduce the amount the Si WG output diffracts outward. However, care must be taken to not allow the fluid to flow over on top of the polymer taper to maintain its high NA.

\subsubsection{Metallic Loss}

A metallic-coated substrate can be used as a lower cladding for polymer tapers to ensure efficient coupling with high NA Si WGs while eliminating substrate radiation. As shown 
by Kaminow [99] and Rashleigh [100] propagation loss for metal-clad polymer WG will experience polarization-dependent loss due to absorption of the evanescent wave in the metal. Attenuation of the $\mathrm{TE}_{0}$ mode from a gold lower-cladding is high $(260 \mathrm{~dB} / \mathrm{cm}, \lambda=$ $1310 \mathrm{~nm})$ for small $(0.5 \mu \mathrm{m}) \mathrm{WG}$ heights and decreases exponentially as the waveguide height is increased. The $\mathrm{TM}_{00}$ mode couples to the surface plasmon wave and mostly resides at the metal-dielectric boundary resulting in extremely high $(\sim 1650 \mathrm{~dB} / \mathrm{cm}, \lambda=$ $1310 \mathrm{~nm}$ ) attenuation independent of WG dimensions. Both mode profiles are illustrated in Figure 6.20. For this reason metal-clad WGs have been used in integrated optics as polarization filters with high extinction ratios [107, 108]. Metallic-bottom clad polymer WG tapers will be optimal for TE mode conversion due to the high absorption loss of TM polarized light from plasmonic coupling. Shallow-etched Si rib WGs inherently propagate the $\mathrm{TE}_{00}$ mode with low loss allowing for polarization compatibility with metallic-bottom tapers [109].

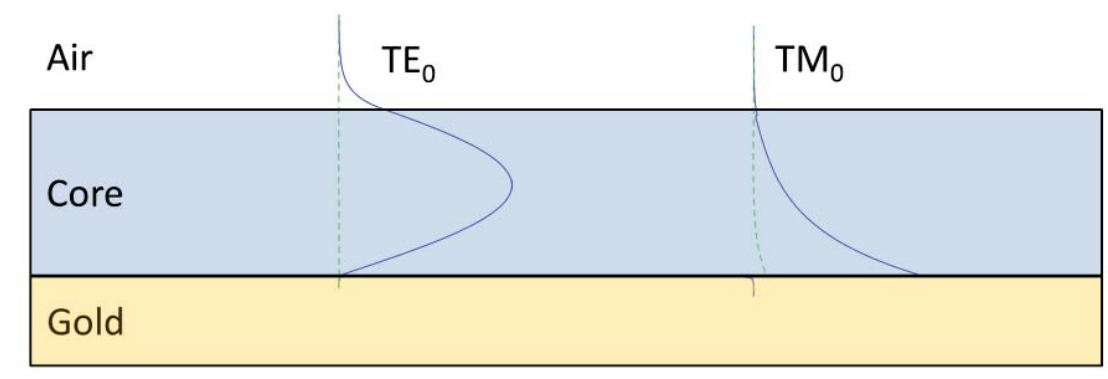

Figure 6.20 Mode profile of the $T E_{0}$ and $T M_{0}$ of an asymmetrical gold/polymer/air Waveguide. 
Device length of polymer tapers with a gold-bottom clad should be optimized to minimize taper device loss. Metallic loss can be assessed as the integration of WG height along the WG taper and the $\mathrm{TE}_{00}$ attenuation as a function of WG height. Polymer taper length should be close to the adiabatic length constraint to balance both adiabatic taper loss (too short) and propagation loss from metallic absorption (too long). Multi-layer tapers should also be fabricated with the middle taper length equal to the total taper length, as illustrated in Figure 6.6 ensuring the coupled mode does not experience additional loss ( $\geq 90 \mathrm{~dB} / \mathrm{cm})$ while propagating through a sub-micron $(\leq 1 \mu \mathrm{m}) \mathrm{WG}$ height.

\subsection{Polymer WG Taper Experimental Results}

Polymer WG taper prototypes were fabricated through photolithography and evaluated through optical testing to evaluate both taper loss and coupling loss and show comparability to theoretical losses. Polymer WG tapers were first measured for taper loss to correlate with theoretical loss associated metallic absorption and sidewall roughness scattering. Coupling loss between polymer tapers and Si WGs were measured and compared to theoretical coupling loss due to mode overlap and interface reflection.

\subsubsection{Taper Fabrication}

Multi-layer taper prototyping was done utilizing a multi-step photolithographic process [104] utilizing the UV-curable optical elastomer manufactured by Dow Corning ${ }^{\circledR}$. Photolithography was the chosen manufacturing method due to its mature technology, 
CMOS compatibility and accessibility in the industrial sector [16, 38]. Tapers were fabricated on a gold-sputtered silicon substrate to prevent substrate radiation modes. Gold was chosen for its high reflectivity, corrosion resistance, and low attenuation loss [110]. The overall taper design was $2 \mathrm{~mm}$ in length to ensure adiabatic modal expansion. The bottom layer utilized a diluted polymer solution ( $75 \%$ solvent) to spin coat a $0.7 \mu \mathrm{m}$ thick layer to maintain SM functionality as determined by the created spin curve in Figure 6.21. This layer was patterned into $6 \mu \mathrm{m}$ WGs that taper down to $2 \mu \mathrm{m}$ taper widths for optimal coupling with wider Si WGs with a mode profile shown in Figure 6.11(b). The second and third elastomer layers were spun to a thickness of $3 \mu \mathrm{m}$ and $6 \mu \mathrm{m}$ and patterned into overlying tapered WGs of shorter lengths of $1.6 \mathrm{~mm}$ and $1.2 \mathrm{~mm}$ respectively, as shown in Figure 6.22. Multi-layer taper end-faces were prepared using traditional Si wafer cleaving.

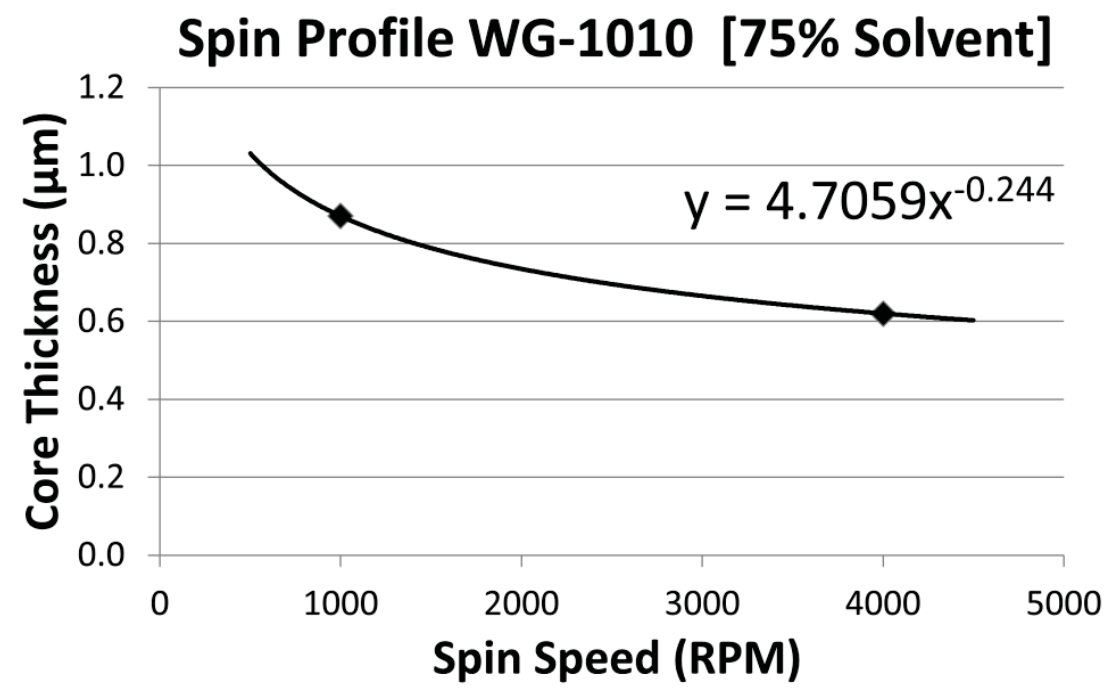

Figure 6.21 Spin Curve of diluted (75\% solvent) UV-curable optical elastomer utilized in polymer $W G$ tapers. 


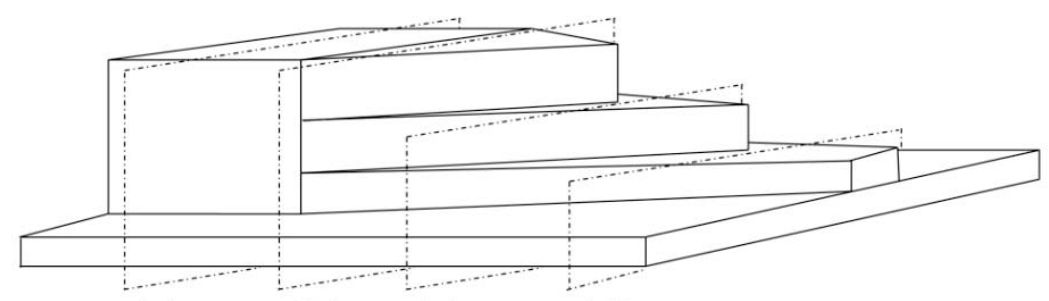

(a)

(b)

(d)

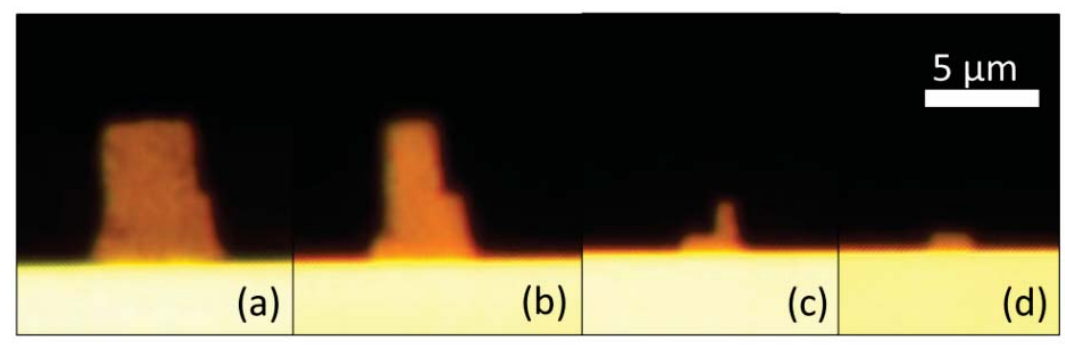

Figure 6.22 Cross sections of fabricated multi-layer taper along the length of the polymer $W G$ device.

\subsubsection{Taper Loss}

The device loss of multi-layer tapers were measured with TE polarized light at an operating wavelength of $\lambda=1310 \mathrm{~nm}$. The $6 \mu \mathrm{m}$ taper end was end-fire coupled with a SMF and the output of the taper was captured with a microscope objective $(\mathrm{NA}=0.85)$ and measured using a Germanium photodiode. The evaluation setup is shown in Figure 6.23. The measured taper loss for an average of four polymer WG tapers was measured at $15.1 \pm 0.7 \mathrm{~dB}$ in comparison to the simulated taper loss of $12.0 \mathrm{~dB}$ discussed below in Section 6.4.4. Taper loss can be significantly reduced utilizing higher mask resolutions 
and improving manufacturing techniques to reduce sidewall scattering and metallic absorption.

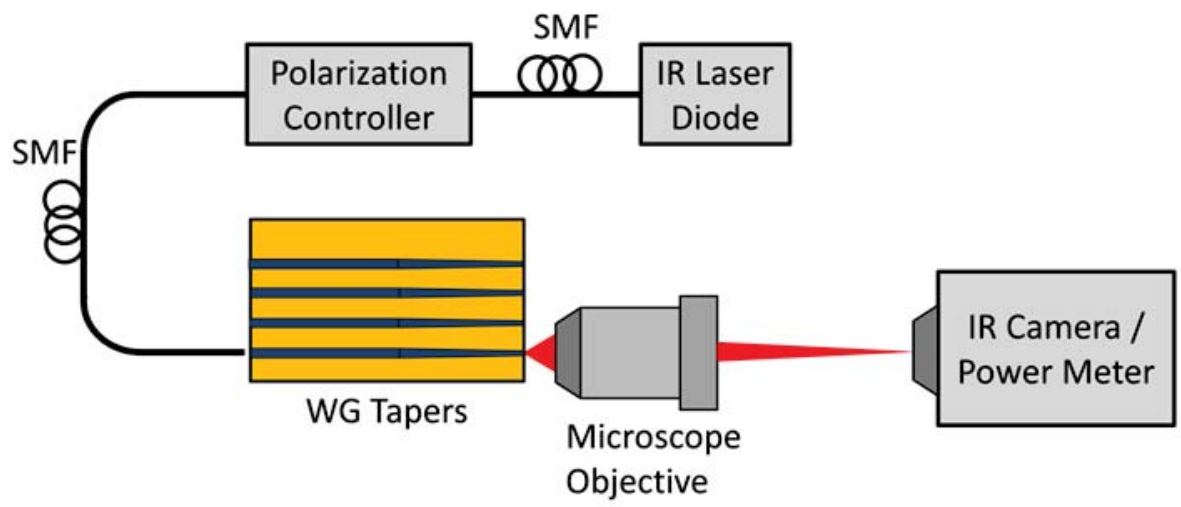

Figure 6.23 Measurement setup used to determine taper loss due to sidewall roughness and gold absorption.

\subsubsection{Si WG Coupling}

Coupling loss between Si WGs and polymer tapers was also evaluated. TE-polarized laser diode power $(\lambda=1310 \mathrm{~nm})$ was coupled into Si wire WGs $(2 \mu \mathrm{m} \times 0.215 \mu \mathrm{m})$ using a lensed SMF ( $2 \mu \mathrm{m}$ spot size) as an optical input. Index-matching fluid $(n=1.512)$ was applied between actively aligned polymer tapers and Si WGs to reduce boundary reflection and air-gap loss. Output power was coupled using a microscope objective (NA $=0.85$ ) and measured using a Germanium photodiode. The measurement setup in determining coupling loss is shown in Figure 6.24. 


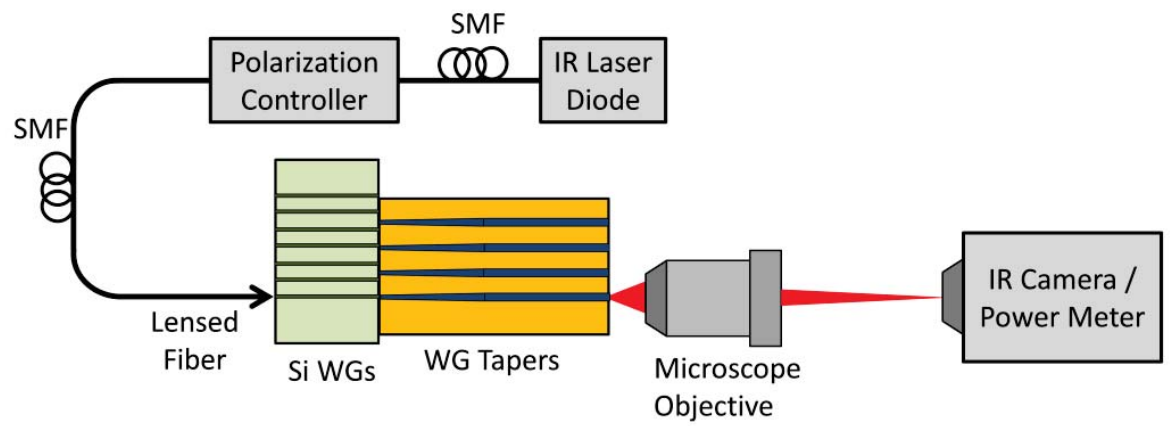

Figure 6.24 Measurement setup used to determine polymer taper coupling loss with Si WGs.

Coupling loss between polymer tapers and Si WGs was measured at $2.74 \pm 1.0 \mathrm{~dB}$ after taking into account the measured taper loss $(15.1 \mathrm{~dB})$ assuming symmetric modal propagation theoretically shown in Figure 6.9. This is comparable to the theoretical coupling loss of $2.48 \mathrm{~dB}$ due to the summation of mode overlap loss $\left(\Gamma_{C}=1.72 \mathrm{~dB}\right)$ and theoretical Fresnel reflection loss $(0.76 \mathrm{~dB})$ at the Silicon-polymer interface. Coupling loss due to interface reflection can be further reduced by utilizing appropriate index matching techniques and anti-reflection coatings at the Si-polymer boundary.

\subsubsection{Polymer Taper Loss Optimization}

While the coupling loss between polymer WG tapers and Si WGs is comparable to theoretical results a large divide between ideal and experimental taper loss is experimentally evaluated. This high level of taper loss is due to a combination of gold absorption and sidewall scattering. 
The polymer WG taper prototype will theoretically experience high taper loss partially due to excessive levels of metallic absorption $(4.9 \mathrm{~dB})$. Precision limitations in prototype cleaving resulted in polymer tapers exhibiting a $0.7 \mu \mathrm{m}$ WG height $250 \mu \mathrm{m}$ long beyond the WG taper, shown in Figure 6.22(d). This polymer WG region results in a theoretical gold absorption loss of $2.5 \mathrm{~dB}$ in addition to the $2.4 \mathrm{~dB}$ absorption loss in the polymer taper. Device preparation methods with greater accuracy can reduce this regional loss by minimizing the length of the region represented by Figure $6.22(\mathrm{~d})$. Gold loss in simulated polymer WG tapers is observed to be a function taper length (absorption distance) and taper height (evanescent wave overlap with gold cladding), as shown in Table 6.1. Gold absorption loss in multi-layer tapers is reduced further $(1.0 \mathrm{~dB})$ when the taper length is manufactured at the adiabatic length $(600 \mu \mathrm{m})$. While gold loss is reduced with increasing taper height this is counteracted with theoretically lower coupling loss shown previously in Figure 6.15.

Table 6.1 Theoretical gold absorption loss of fabricated multi-layer taper as a function of taper height and taper length.

\begin{tabular}{r|rrr} 
Taper Length & $\mathbf{0 . 5} \mu \mathrm{m}$ Height & $\mathbf{0 . 7} \boldsymbol{\mu m}$ Height & $\mathbf{1 . 0} \boldsymbol{\mu m}$ Height \\
\hline $600 \mu \mathrm{m}$ & $2.2 \mathrm{~dB}$ & $1.0 \mathrm{~dB}$ & $0.7 \mathrm{~dB}$ \\
$1000 \mu \mathrm{m}$ & $2.3 \mathrm{~dB}$ & $1.7 \mathrm{~dB}$ & $1.1 \mathrm{~dB}$ \\
$2000 \mu \mathrm{m}$ & $4.1 \mathrm{~dB}$ & $3.2 \mathrm{~dB}$ & $2.1 \mathrm{~dB}$
\end{tabular}

High-NA waveguides are more susceptible to scattering loss from the level of sidewall roughness resulting from the fabrication method. Surface roughness loss increases in 
WGs with higher NA and smaller dimensions [111].The high degree of sidewall roughness can easily be observed in Figure 6.25. The sidewall roughness of polymer WGs fabricated through photolithography has previously been measured at $48 \mathrm{~nm}$ RMS with a correlation length $\left(L_{c}\right)$ of $3 \mu \mathrm{m}$ [42]. Polymer WG scatting also exponentially scales with decreasing polymer WG width, as shown in Figure 6.26. BPM simulations estimate the scattering loss of the taper device at $7.1 \mathrm{~dB}$. Device scattering loss can be significantly reduced $(0.7 \mathrm{~dB})$ by both reducing taper length $(600 \mu \mathrm{m})$ and device sidewall roughness ( $25 \mathrm{~nm}$ RMS) shown in Table 6.2. While the mask details for these layers taper down to $0.5 \mu \mathrm{m}$, the patterned features are diffraction-limited at $1 \mu \mathrm{m}$. The resulting tapers are under-exposed, exhibit a fluctuating structure that flatten out at the taper's end, as shown in Figure 6.27.
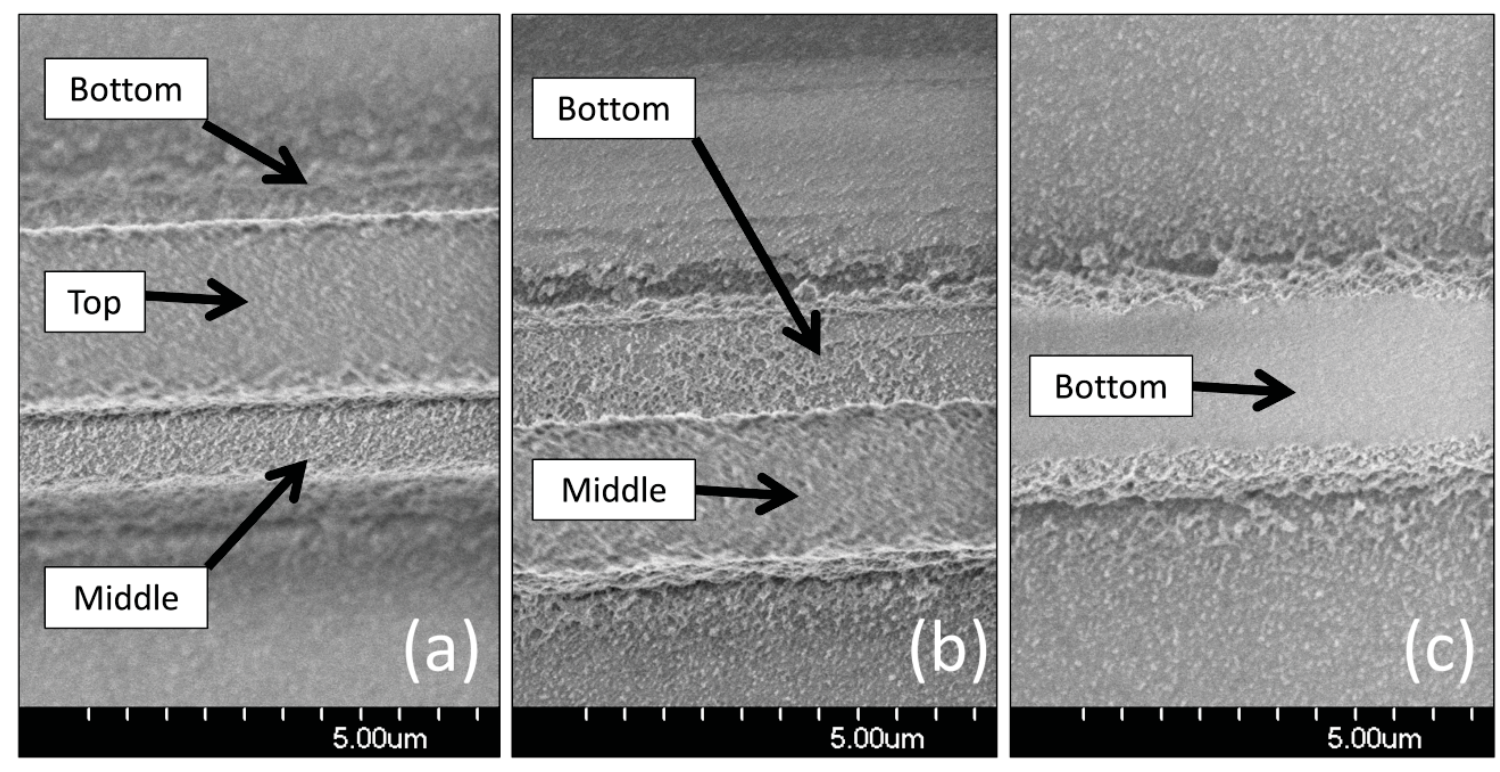

Figure 6.25 FE-SEM images of multi-layer polymer tapers with 3 layers (a), 2 layers (b), and 1 layer (c). 


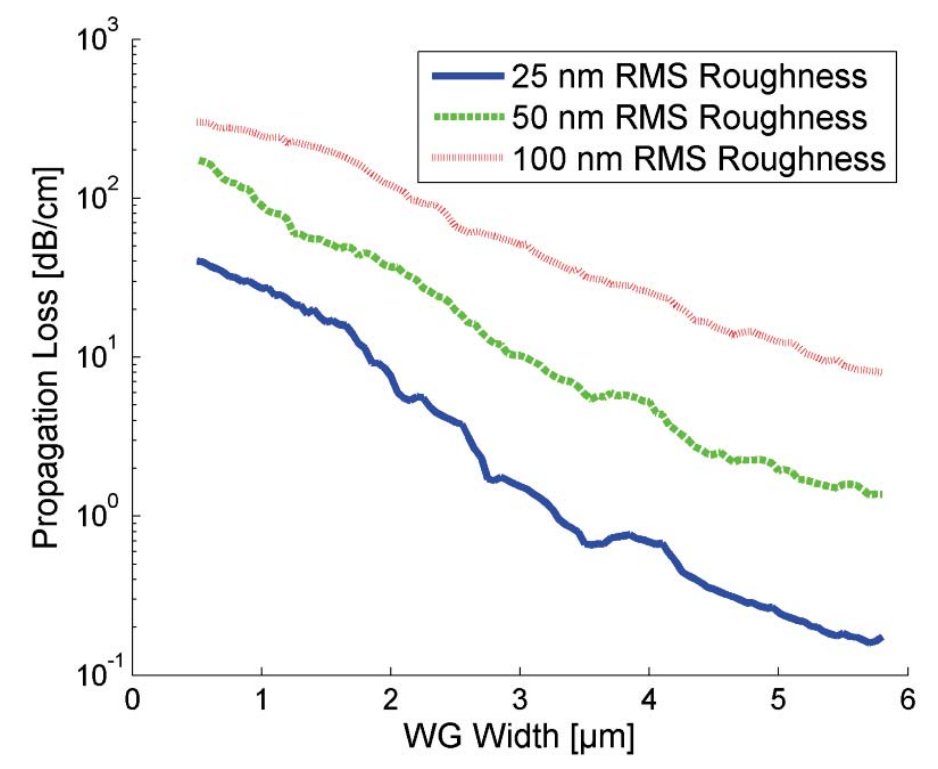

Figure 6.26 Simulated propagation loss from sidewall scattering for air-clad $W G$ s as a function of $W G$ width and sidewall roughness $\left(\lambda=1310 \mathrm{~nm}, L_{c}=3 \mu \mathrm{m}\right)$.

Table 6.2 Theoretical roughness loss of fabricated multi-layer taper as a function of taper length and surface roughness.

\begin{tabular}{r|rrr} 
Taper Length & $\mathbf{2 5} \mathbf{~ n m ~ R M S}$ & $\mathbf{5 0} \mathbf{n m}$ RMS & $100 \mathbf{~ n m ~ R M S ~}$ \\
\hline $\mathbf{6 0 0 \mu \mathrm { m }}$ & $0.7 \mathrm{~dB}$ & $2.2 \mathrm{~dB}$ & $8.9 \mathrm{~dB}$ \\
$\mathbf{1 0 0 0 \mu \mathrm { m }}$ & $0.8 \mathrm{~dB}$ & $3.3 \mathrm{~dB}$ & $11.6 \mathrm{~dB}$ \\
$\mathbf{2 0 0 0} \boldsymbol{\mu m}$ & $1.9 \mathrm{~dB}$ & $7.1 \mathrm{~dB}$ & $16.9 \mathrm{~dB}$
\end{tabular}

Total device loss can be reduced further by utilizing vertical tapers as coupling devices since their shorter adiabatic length requirement $(200 \mu \mathrm{m})$ allows for lower levels of length-dependent propagation loss. Vertical tapers manufactured with $25 \mathrm{~nm}$ RMS sidewall roughness would exhibit device loss of $1.1 \mathrm{~dB}$ as a result of gold absorption (1.0 $\mathrm{dB})$ and scattering $(0.1 \mathrm{~dB})$, as detailed in Table 6.3 and Table 6.4. 


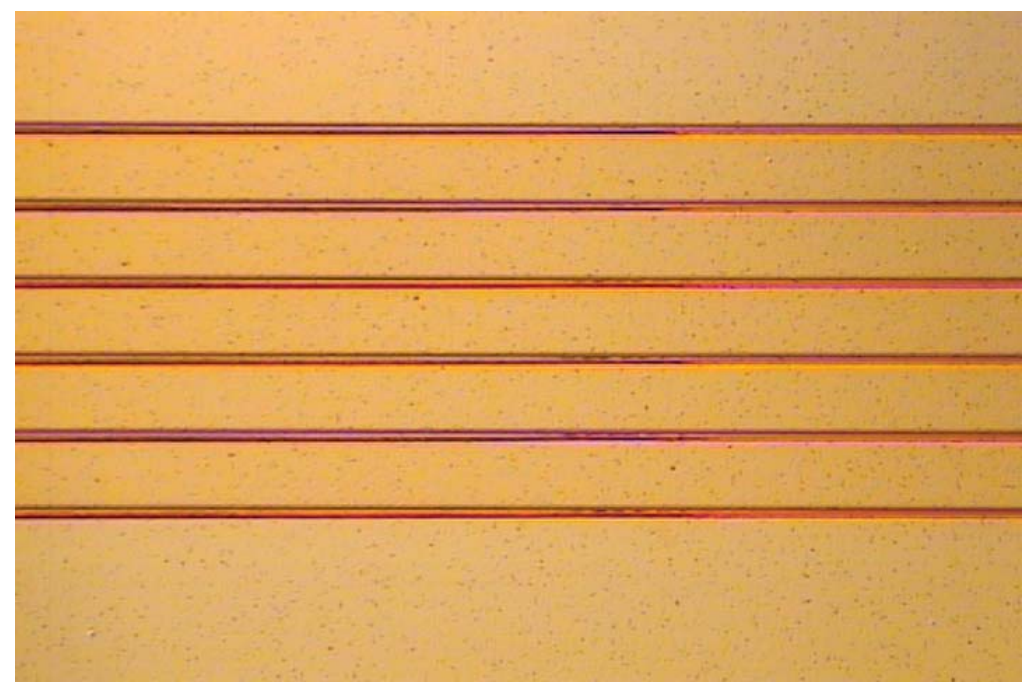

Figure 6.27 Overlapping tapers exhibiting under-exposure effects from diffraction-limited fabrication.

Table 6.3 Theoretical gold absorption loss of vertical taper as a function of taper height and taper length.

\begin{tabular}{r|rrr} 
Taper Length & $\mathbf{0 . 5} \boldsymbol{\mu m}$ Height & $\mathbf{0 . 7} \boldsymbol{\mu m}$ Height & $\mathbf{1 . 0} \boldsymbol{\mu m}$ Height \\
\hline $200 \mu \mathrm{m}$ & $1.5 \mathrm{~dB}$ & $1.0 \mathrm{~dB}$ & $0.7 \mathrm{~dB}$ \\
$1000 \mu \mathrm{m}$ & $2.9 \mathrm{~dB}$ & $2.4 \mathrm{~dB}$ & $0.9 \mathrm{~dB}$
\end{tabular}

Table 6.4 Theoretical sidewall roughness loss of vertical taper as a function of sidewall roughness and taper length.

\begin{tabular}{r|rrr} 
Taper Length & $\mathbf{2 5} \mathbf{n m}$ RMS & $\mathbf{5 0} \mathbf{~ n m ~ R M S ~}$ & $\mathbf{1 0 0} \mathbf{~ n m ~ R M S ~}$ \\
\hline $\mathbf{2 0 0 \mu \mathrm { m }}$ & $0.1 \mathrm{~dB}$ & $0.2 \mathrm{~dB}$ & $0.3 \mathrm{~dB}$ \\
$1000 \mu \mathrm{m}$ & $0.1 \mathrm{~dB}$ & $0.6 \mathrm{~dB}$ & $3.0 \mathrm{~dB}$
\end{tabular}

Both taper designs can be fabricated with one-step procedures eliminating alignmentbased processing steps and decreasing overall design costs utilizing master molds. Soft 
imprint lithography is a high-resolution $(<10 \mathrm{~nm})$, high-throughput molding technique for polymer materials that exhibit thermoplastic characteristics, making it incompatible with complex network polymers or materials with high amounts of cross-linking [15, $112,113]$. Step-and-flash imprint lithography has been shown to be reliable method for molding complex polymer materials at micron resolution through the use of a transparent mold [114]. Utilizing photolithographic materials that polymerize and thermally cure with the assistance of an UV initiator, WG polymers can be uniformly UV cured, pressed into the desired taper shape, and then thermally cured to complete polymerization without the requirement of a transparent mask [115].

\subsection{WG Taper Bridge Module}

\subsubsection{Module Design}

Polymer bridge modules must conduct efficient mode conversion and coupling efficiency between dissimilar optical waveguiding devices. Another factor that requires consideration when bridging between distinct waveguiding devices is pitch density control. Si WGs are capable of high bandwidth layouts with minimal crosstalk when $\mathrm{Si}$ WGs exhibit pitches of $<5 \mu \mathrm{m}$ [116] which is significantly smaller than the width of traditional SMFs $(125 \mu \mathrm{m})$. Polymer bridges must allow for WG pitch control while optimizing optical coupling and minimizing cross-talk. Polymer WG S-bends must be incorporated into module designs for optimal pitch control within efficient footprint layouts. 
The polymer bridge design incorporates mode conversion and pitch control for efficient and practical coupling between Si WGs and SMFs. The bridge module utilizes high NA polymer WG tapers for mode coupling and SM polymer WG S-bends for optical density adjustments to obtain a $250 \mu \mathrm{m}$ WG pitch for compatibility with SMF bundles and MT/MPO connector standards [117]. A coupling bridge allows for connectorization between Si WGs and SMFs without the expense of interconnect end-face preparations by utilizing flat end-faces at both interfaces. The polymer bridge module layout is shown in Figure 6.28.

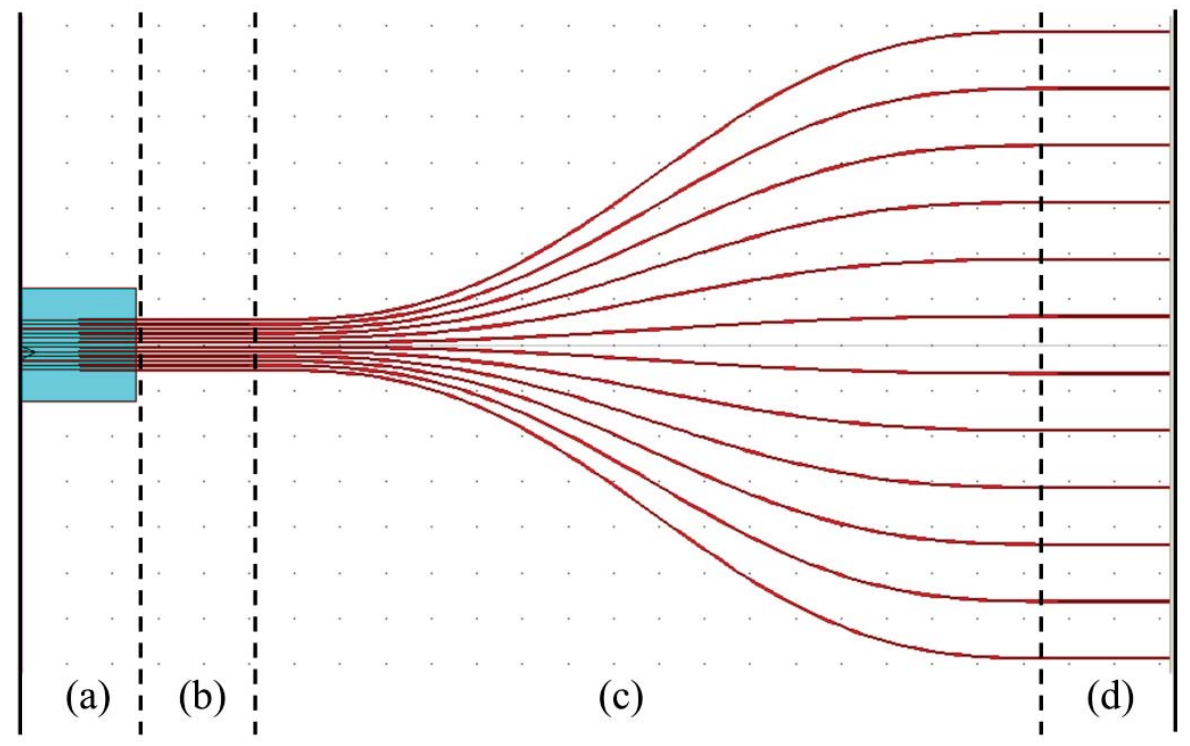

Figure 6.28 Polymer taper bridge module incorporated with WG tapers (a), isolation WGs (b) WG S-Bends (c), and end WGs (d).The shaded region in section (a) represents a low-index medium for high $N A W G$ tapers.

After modal expansion through the WG taper the optical signal is then transferred into SM polymer WGs. Slight modal mismatch is experienced between polymer WG tapers 
and SM WG (1.1 dB). Stray light not coupled into polymer WG tapers will be present within bridge module. Isolation WG straights located between tapers and S-bends isolate the coupling region from the pitch control reduce stray light coupling in WG bends. The length of isolation WGs should be optimized to reduce modal coupling from stray light (too short) and neighboring WGs (too long).

Once the optical power is coupled into the polymer WG the optical WG pitch can be adjusted to match those of commercially available SM connectors utilizing WG S-bends. Cosine S-bends are optimal for module design for their low structure loss at reduced footprint requirements. Cosine S-bends also exhibit the highest bend radius at its endfaces minimizing the mode coupling length between neighboring WGs during pitch control.

Straight WG ends can be incorporated after polymer WG S-bends. These WGs exhibits no length requirements allowing designers the flexibility to expand the module to required standards and can be cut and polished at any location without altering the module's coupling capabilities. Minimal $(0.06 \mathrm{~dB})$ coupling loss and large $( \pm 3.5 \mu \mathrm{m})$ alignment tolerances are expected at the module/SMF interface from experimental results shown in Chapter 3.

\subsubsection{Module Crosstalk}

It is important to ensure that leaked power does not couple into neighboring waveguides in the form of crosstalk and signal noise. Isolation WG straights were incorporated in 
simulated design models between inverse coupling tapers and WG S-bends. However, isolation WGs will exhibit cross-talk as mode coupling will be present between parallel waveguide straights and becomes extensive when matched with extremely dense Si WGs. A minimum crosstalk limit of $-30 \mathrm{~dB}$ is highly desired due to industrial standards [118]. With a Si WG pitch of $25 \mu \mathrm{m}$, RSoft BeamPROP simulations were conducted to observe crosstalk levels with different lengths of isolation WGs due to power leakage and undesired mode coupling.

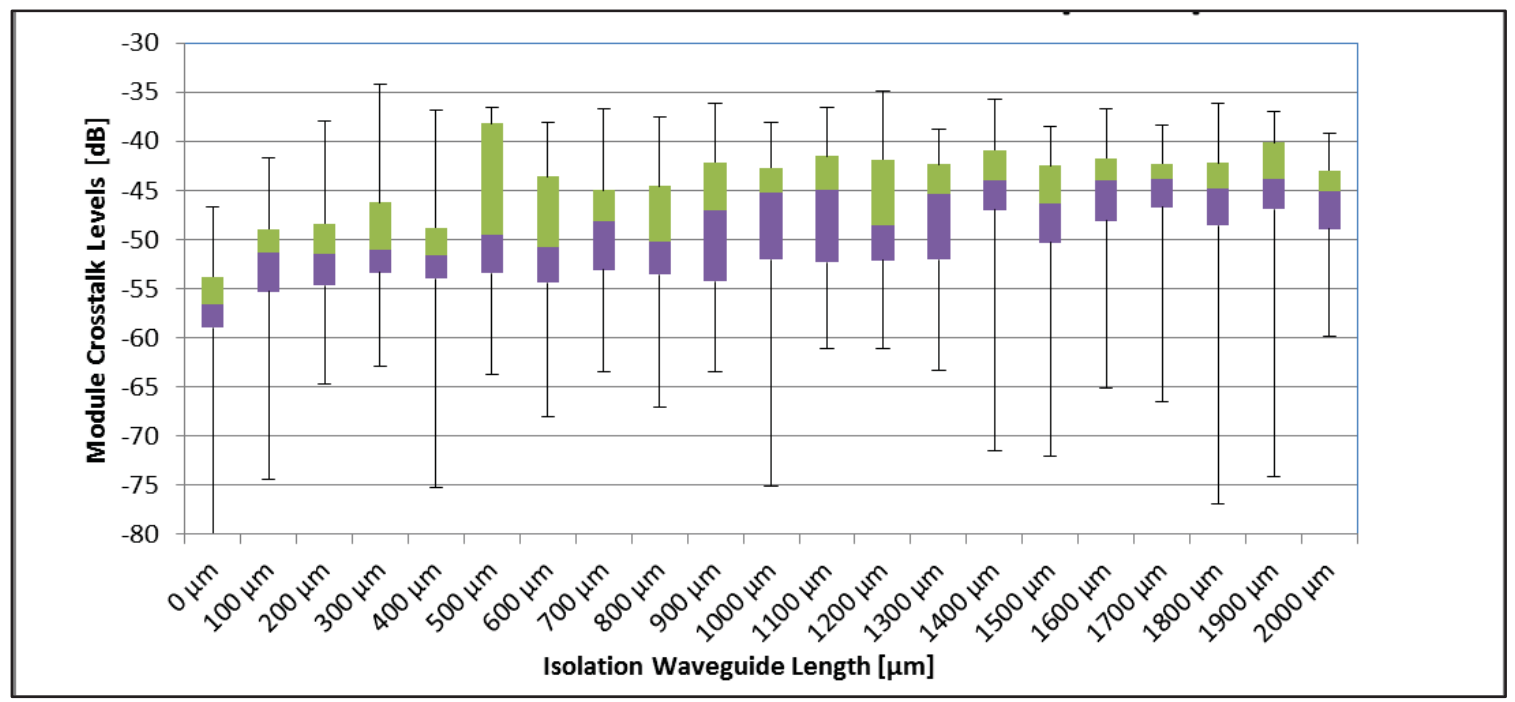

Figure 6.29 Waveguide module cross-talk levels over multiple individually excited WGs. Box plots show the Q1, mean, and Q3 levels of cross-talk in simulation. Whiskers show full range of the data.

Cross-talk levels in simulations stayed below $-35 \mathrm{~dB}$ throughout the coupler module, as shown in Figure 6.29. While the individual cross-talk levels over the various waveguides are very inconsistent, a gradual increase in cross-talk levels exists as the isolation WG 
lengths increase. An optimal isolation WG length to minimize cross-talk levels was not apparent demonstrating that mode coupling between isolation WGs has a greater impact than inverse taper cross-talk. Results show that cross-talk is minimized if isolation WGs are omitted.

\subsubsection{Module Layout and Losses}

The size of the optical coupler module will ultimately be constrained by the parameters of the SMF device. The main goal of this research has been set on coupling light from Si WGs to MT connecters, $12 \mathrm{SMFs}$ separated by a $250 \mu \mathrm{m}$ pitch. This restricts the module's minimum width requirement to $3 \mathrm{~mm}$. The overall length of the coupler will be the summation of the individual distance requirements for the module sections shown previously in Figure 6.28. Vertical polymer WG tapers require an adiabatic length requirement of $0.2 \mathrm{~mm}$. Isolation WGs shown in Figure 6.28(b) were omitted to optimize device cross-talk. The majority of the module's size is dependent on by the S-bends length requirements for maximum device efficiency. The outermost SM WG S-bends in the module have to spread outward a maximum distance of $1.38 \mathrm{~mm}$ from the center to couple with MT connectors. This fan-out requires S-bend WGs to be manufactured with a length of $6.5 \mathrm{~mm}$ to optimize both structure loss and propagation loss. By adding this value to the length requirement of the inverse tapers, the overall dimensions for the proposed optical module couple are $3.0 \mathrm{~mm}$ x $6.7 \mathrm{~mm}$.

The total module loss is the combination of the taper and interface losses of the WG taper For example, coupling from Si Wire WGs to SMF would result in a bridge loss of $5.8 \mathrm{~dB}$ 
due to Si Wire coupling (3.0 dB), optimal taper loss from gold absorption and sidewall roughness scattering $(1.1 \mathrm{~dB})$, SM WG coupling $(1.1 \mathrm{~dB})$, S-bend loss $(0.5 \mathrm{~dB})$, and SMF coupling $(0.1 \mathrm{~dB})$. This result is a strong improvement over the $18.8 \mathrm{~dB}$ loss when directly coupling Si WGs and SMFs. The majority of coupling loss is dependent on the coupling loss at the Si WG interface and is significantly reduced when coupling to larger mode-field diameter waveguiding devices including Si rib WGs (4.2 dB).

\subsection{WG Taper Conclusion}

Mode conversion devices are vital in optimizing data transmission rates and coupling efficiencies into and out of Si photonic applications. Two commercially producible WG taper designs are proposed and modeled to provide minimal coupling and misalignment loss for optical interconnects. Vertical tapers and multi-layer tapers couple the highly divergent $\mathrm{Si}$ WG output and expand the mode profile to improve coupling with low NA devices. Both taper designs have been evaluated as compatible for coupling with both $\mathrm{Si}$ nanowire WGs and Si rib WGs.

Device dimensions for both designs were analyzed through BPM simulations $(\lambda=1310 \mathrm{~nm})$ for coupling with Si photonic interconnects. Taper length requirements for adiabatic mode conversion were evaluated at $200 \mu \mathrm{m}$ and $600 \mu \mathrm{m}$ for vertical and multilayer tapers respectively. Minimum bridge loss with Si Wire WGs (4.2d dB) and Si Rib (2.75 dB) WGs was observed with $0.6 \mu \mathrm{m}$ and $2.0 \mu \mathrm{m}$ taper widths respectively. Widening taper widths were observed to increase $3 \mathrm{~dB}$ misalignment tolerances at the expense of decreasing maximum coupling efficiency. 
Multi-layer WG tapers were fabricated with multi-step photolithography on a goldsputtered substrate. Device loss was measured at $15.1 \mathrm{~dB}$ for $2 \mathrm{~mm}$ long multi-layer tapers with strong correlation to a theoretical loss due to metallic absorption $(5.0 \mathrm{~dB})$ and sidewall roughness (7.1). Taper device loss for practical builds can be reduced by implementing reduced taper lengths, reducing sidewall roughness, and improving endface cleaving accuracy. Coupling loss between multi-layer tapers and Si WGs was measured at $2.74 \mathrm{~dB}$ with high correlation to the theoretical coupling loss of $2.5 \mathrm{~dB}$ due to mode overlap and interface reflection.

Polymer WG tapers were incorporated with SM WG S-bends for bridge module design for optimizing optical coupling and pitch control. Module design requirements were studied to optimize size requirements and device loss. Cross-talk was also analyzed and minimized when isolation WGs were omitted from the module design. Polymer bridge modules require a footprint of $3 \mathrm{~mm} \times 5.8 \mathrm{~mm}$ for optimal coupling performance. Coupling between Si wire WGs and MT cables require device dimensions of $3.0 \mathrm{~mm} \mathrm{x}$ $6.7 \mathrm{~mm}$ and operate at a theoretical $5.8 \mathrm{~dB}$ bridge loss in contrast to direct coupling (18.8 $\mathrm{dB})$. 


\section{Chapter 7: Conclusion}

\subsection{Research Summary}

The replacement of copper interconnects with optical interconnects is a commercially viable solution to support data transmission rates in the telecommunications industry. Optical interconnects including polymer WGs hold promise in enhancing computational power, reducing power requirements, and increasing communication speeds for both current and next-generation data networks. An analysis on the design, fabrication, and connectorization of SM polymer WG designs and components for short-haul optical interconnect applications with strong emphasis on a novel, all-optical bridge module design has been presented.

SM polymer WGs were fabricated through both photolithography and laser direct writing (LDW) and evaluated demonstrating low $(0.6 \mathrm{~dB} / \mathrm{cm})$ propagation loss, efficient $(0.06$ $\mathrm{dB} /$ end-face) optical coupling with SMFs, and functional at high data rates (>10 Gbits/s). The LDW method with a flexible programmable interface was developed as a costeffective prototyping method for manufacturing polymer WGs with rigid rectangular structures, low $(0.55 \mathrm{~dB} / \mathrm{cm})$ propagation loss, and high repeatability within $<5 \%$ manufacturing error. A novel analytical model was derived to successfully correlate polymer WG dimensions to LDW parameters to determine optimal writing constraints to fabricate low loss LDW MM and SM polymer WGs. Optimal manufacturing parameters were obtained for the LDW of symmetric low-loss MM WGs $(0.035 \mathrm{~dB} / \mathrm{cm}, \lambda=830 \mathrm{~nm})$ 
and SM WGs $(0.55 \mathrm{~dB} / \mathrm{cm}, \lambda=1310 \mathrm{~nm})$ polymer WGs. Polymer WG straight, radial bends, and crossings were also evaluated and shown to be comparable between the two fabrication methods. The LDW method was shown through optical testing as a viable alternative to polymer WG manufacturing with comparable performance levels to photolithographic polymer WGs.

SM WG S-bend designs were investigated for their importance for realizing high-density optical interconnects for multiple applications. S-bend structures are thoroughly analyzed to realize optimal S-bend design for WGs of various NA and dimensions. LDW polymer WG S-bend designs were also fabricated indirectly using the segmentation method for compatibility with G-code. Optimal segmentation length $\left(L_{s}=10-50 \mu \mathrm{m}\right)$ exhibited the lowest device loss for segmented LDW S-bends $(25 \mathrm{~mm} / \mathrm{s})$. Cosine S-bends were experimentally shown to exhibit the lowest loss for both photolithographic and LDW builds. Optical splitters were fabricated with $1 \mathrm{~dB}$ and $3 \mathrm{~dB}$ S-bends exhibiting splitting ratios of 49:51 $\pm 2 \%$. Optical splitters were also successfully fabricated with adiabatic tapers to both maximize splitting efficiency and minimize device footprint requirements.

Two novel, commercially viable WG taper designs, vertical and multi-layer tapers, have been proposed and investigated for optical interconnects. Theoretical work has been laid out for the designs requirements for polymer WG tapers as an optical coupling bridge between sub-micron Silicon WGs and SMFs. Taper length requirements for adiabatic mode conversion was evaluated for both vertical $(200 \mu \mathrm{m})$ and multi-layer $(600 \mu \mathrm{m})$ tapers designs. Optimal taper dimensions are acquired through BPM simulations for 
efficient coupling with Si Wire WGs $(4.2 \mathrm{~d} d \mathrm{~B})$ and $\mathrm{Si}$ Rib $(2.75 \mathrm{~dB})$ WGs with $0.6 \mu \mathrm{m}$ and $2.0 \mu \mathrm{m}$ taper widths respectively. Multi-layer tapers were fabricated and evaluated for structure and coupling loss. Device loss was correlated to theoretical metallic absorption and sidewall roughness scattering. Taper device loss for practical builds can be reduced by implementing reduced taper lengths, reducing sidewall roughness, and improving end-face cleaving accuracy. Coupling loss $(2.74 \mathrm{~dB})$ between multi-layer tapers and Si WGs showed high correlation to theoretical coupling loss (2.5 dB).

\subsection{Future Work}

Though this work adds a substantial amount of progress towards the commercialization and feasibility of polymer WGs as short-haul optical interconnects, additional theoretical techniques could be pursued to further improve the manufacturing efficiency of polymer WGs for additional commercial applications.

Efficient methods to apply thin, uniform layers of optical material can increase the overall manufacturing speed of large-panel optical interconnect designs. The LDW manufacturing platform can be incorporated with alternative polymer-coating methods including doctor blading and ink-jet printing to improve polymer WG fabrication and reduce optical interconnect manufacturing costs.

The acquired knowledge on optimal S-bend design can be utilized and incorporated in the design of compact active optical components in the form of mode couplers and MachZehnder interferometers. Various S-bend designs can be experimentally evaluated for 
high-density WGs including silicon photonic interconnects. While work has been done on these applications research on optimizing these designs can further contribute to their potential as cutting-edge device in the commercial market.

More work is required to maximize taper bridge loss for optimal coupling bridge loss between SMF and Si WGs. Taper sidewall roughness needs to be reduced through highresolution fabrication methods. Multi-layer tapers can be manufactured with highthroughput techniques utilizing soft and nano-imprint lithography utilizing low-roughness $(<25 \mathrm{~nm}$ RMS) master molds. Advanced fabrication methods can be implemented into functional vertical tapered WGs to improve device efficiency and reduce size requirements. 


\section{References}

[1] D. Huang, T. Sze, A. Landin, R. Lytel, and H. L. Davidson, "Optical interconnects: out of the box forever?," Selected Topics in Quantum Electronics, IEEE Journal of, vol. 9, pp. 614-623, 2003.

[2] A. F. Benner, M. Ignatowski, J. A. Kash, D. M. Kuchta, and M. B. Ritter, "Exploitation of optical interconnects in future server architectures," IBM Journal of Research and Development, vol. 49, pp. 755-775, 2005.

[3] C. Berger, M. A. Kossel, C. Menolfi, T. Morf, T. Toifl, and M. L. Schmatz, "High-density optical interconnects within large-scale systems," in Photonics Fabrication Europe, 2003, pp. 222-235.

[4] P. K. Pepeljugoski, J. A. Kash, F. Doany, D. M. Kuchta, L. Schares, C. Schow, et al., "Low power and high density optical interconnects for future supercomputers," in Optical Fiber Communication Conference, 2010, p. OThX2.

[5] G. Keiser, Optical fiber communications: Wiley Online Library, 2003.

[6] G. Khoe and G. Kuyt, "Realistic efficiency of coupling light from GaAs laser diodes into parabolic-index optical fibres," Electronics Letters, vol. 14, pp. 666$668,1978$.

[7] M. Immonen, M. Karppinen, and J. K. Kivilahti, "Fabrication and characterization of polymer optical waveguides with integrated micromirrors for threedimensional board-level optical interconnects," Electronics Packaging Manufacturing, IEEE Transactions on, vol. 28, pp. 304-311, 2005.

[8] J. Dingeldein, K. L. Kruse, C. Demars, C. Middlebrook, C. Friedrich, and M. Roggemann, "Optical-electrical printed wiring board for high-speed computing applications," Optical Engineering, vol. 52, pp. 035201-035201, 2013.

[9] R. Dangel, C. Berger, R. Beyeler, L. Dellmann, M. Gmur, R. Hamelin, et al., "Polymer-waveguide-based board-level optical interconnect technology for datacom applications," Advanced Packaging, IEEE Transactions on, vol. 31, pp. 759-767, 2008.

[10] M. Tan, P. Rosenberg, J. S. Yeo, M. McLaren, S. Mathai, T. Morris, et al., "A high-speed optical multi-drop bus for computer interconnections," Applied Physics A, vol. 95, pp. 945-953, 2009.

[11] K. Miura, J. Qiu, H. Inouye, T. Mitsuyu, and K. Hirao, "Photowritten optical waveguides in various glasses with ultrashort pulse laser," Applied Physics Letters, vol. 71, pp. 3329-3331, 1997.

[12] N. Bamiedakis, J. Beals, R. V. Penty, I. H. White, J. V. Degroot, and T. V. Clapp, "Cost-effective multimode polymer waveguides for high-speed on-board optical interconnects," Quantum Electronics, IEEE Journal of, vol. 45, pp. 415-424, 2009.

[13] B. W. Swatowski, C. M. Amb, S. K. Breed, D. J. Deshazer, W. K. Weidner, R. F. Dangel, et al., "Flexible, stable, and easily processable optical silicones for low loss polymer waveguides," in SPIE OPTO, 2013, pp. 862205-862205-11. 
[14] J. Missinne, S. Kalathimekkad, B. Van Hoe, E. Bosman, J. Vanfleteren, and G. Van Steenberge, "Stretchable optical waveguides," Optics express, vol. 22, pp. 4168-4179, 2014.

[15] D. A. Chang-Yen, R. K. Eich, and B. K. Gale, "A monolithic PDMS waveguide system fabricated using soft-lithography techniques," Journal of Lightwave Technology, vol. 23, p. 2088, 2005.

[16] K. Tung, W. Wong, and E. Pun, "Polymeric optical waveguides using direct ultraviolet photolithography process," Applied Physics A, vol. 80, pp. 621-626, 2005 .

[17] A. Liu, R. Jones, L. Liao, D. Samara-Rubio, D. Rubin, O. Cohen, et al., "A highspeed silicon optical modulator based on a metal-oxide-semiconductor capacitor," Nature, vol. 427, pp. 615-618, 2004.

[18] Z. Qiang, W. Zhou, and R. A. Soref, "Optical add-drop filters based on photonic crystal ring resonators," Optics express, vol. 15, pp. 1823-1831, 2007.

[19] C. Gunn, "CMOS photonics for high-speed interconnects," Micro, IEEE, vol. 26, pp. 58-66, 2006.

[20] A. K. Das, B. S. Chaudhari, and S. Ghosh, "Characteristics of polymeric optical passive single-mode waveguiding devices fabricated by an argon-ion laser," Applied optics, vol. 37, pp. 6779-6786, 1998.

[21] F. Tooley, N. Suyal, F. Bresson, A. Fritze, J. Gourlay, A. Walker, et al., "Optically written polymers used as optical interconnects and for hybridisation," Optical materials, vol. 17, pp. 235-241, 2001.

[22] A. K. Das, "Laser direct writing polymeric single-mode waveguide devices with a rib structure," Applied optics, vol. 42, pp. 1236-1243, 2003.

[23] R. R. Krchnavek, G. R. Lalk, and D. H. Hartman, "Laser direct writing of channel waveguides using spin-on polymers," Journal of Applied Physics, vol. 66, pp. 5156-5160, 1989.

[24] F. Ladouceur and P. Labeye, "A new general approach to optical waveguide path design," Lightwave Technology, Journal of, vol. 13, pp. 481-492, 1995.

[25] M. Heinbach, M. Schienle, A. Schmid, B. Acklin, and G. Muller, "Low-loss bent connections for optical switches," Lightwave Technology, Journal of, vol. 15, pp. 833-837, 1997.

[26] F. Mustieles, E. Ballesteros, and P. Baquero, "Theoretical S-bend profile for optimization of optical waveguide radiation losses," Photonics Technology Letters, IEEE, vol. 5, pp. 551-553, 1993.

[27] A. Kumar and S. Aditya, "Performance of S-bends for integrated-optic waveguides," Microwave and Optical Technology Letters, vol. 19, pp. 289-292, 1998.

[28] C. Rolland, G. Mak, K. Fox, D. Adams, A. Springthorpe, D. Yevick, et al., "Analysis of strongly guiding rib waveguide S-bends: theory and experiment," Electronics Letters, vol. 25, pp. 1256-1257, 1989.

[29] P. Buchmann and A. Houghton, "Optical Y-junctions and S-bends formed by preferentially etched single-mode rib waveguides in InP," Electronics Letters, vol. 18, pp. 850-852, 1982. 
[30] L. Hutcheson, I. A. White, and J. J. Burke, "Comparison of bending losses in integrated optical circuits," Optics letters, vol. 5, pp. 276-278, 1980.

[31] W. J. Minford, S. K. Korotky, and R. C. Alferness, "Low-loss Ti: LiNbO 3 waveguide bends at $\lambda=1.3 \mu \mathrm{m}$," Quantum Electronics, IEEE Journal of, vol. 18, pp. 1802-1806, 1982.

[32] A. Sugama, K. Kawaguchi, M. Nishizawa, H. Muranaka, and Y. Arakawa, "Development of high-density single-mode polymer waveguides with low crosstalk for chip-to-chip optical interconnection," Optics express, vol. 21, pp. 24231-24239, 2013.

[33] D. Dai, S. He, and H.-K. Tsang, "Bilevel mode converter between a silicon nanowire waveguide and a larger waveguide," Journal of Lightwave Technology, vol. 24, p. 2428, 2006.

[34] G. Roelkens, P. Dumon, W. Bogaerts, D. Van Thourhout, and R. Baets, "Efficient silicon-on-insulator fiber coupler fabricated using 248-nm-deep UV lithography," IEEE Photonics Technology Letters, vol. 17, pp. 2613-2615, 2005.

[35] T. Alder, A. Stohr, R. Heinzelmann, and D. Jager, "High-efficiency fiber-to-chip coupling using low-loss tapered single-mode fiber," Photonics Technology Letters, IEEE, vol. 12, pp. 1016-1018, 2000.

[36] J. H. van Zantvoort, S. G. Plukker, E. C. Dekkers, G. D. Petkov, G. Khoe, A. M. Koonen, et al., "Lensed fiber-array assembly with individual fiber fine positioning in the submicrometer range," Selected Topics in Quantum Electronics, IEEE Journal of, vol. 12, pp. 931-939, 2006.

[37] M. C. Teich and B. Saleh, "Fundamentals of photonics," Canada, Wiley Interscience, p. 3, 1991.

[38] L. Eldada and L. W. Shacklette, "Advances in polymer integrated optics," Selected Topics in Quantum Electronics, IEEE Journal of, vol. 6, pp. 54-68, 2000.

[39] H. Ma, A. K.-Y. Jen, and L. R. Dalton, "Polymer-based optical waveguides: materials, processing, and devices," Advanced materials, vol. 14, pp. 1339-1365, 2002.

[40] R. Jaszewski, H. Schift, P. Gröning, and G. Margaritondo, "Properties of thin anti-adhesive films used for the replication of microstructures in polymers," Microelectronic Engineering, vol. 35, pp. 381-384, 1997.

[41] R. G. Hunsperger, Integrated optics: theory and technology vol. 2: Springer, 1984.

[42] R. Nicholas, H. Matthew, and M. Christopher, "Optimal VIA Placement in Under Filled Embedded Multimode Waveguides," Optics and Photonics Journal, vol. 2013, 2013.

[43] Z. Sheng, D. Dai, and S. He, "Comparative study of losses in ultrasharp siliconon-insulator nanowire bends," Selected Topics in Quantum Electronics, IEEE Journal of, vol. 15, pp. 1406-1412, 2009.

[44] W. Gambling, D. Payne, and H. Matsumura, "Radiation from curved single-mode fibres," Electronics Letters, vol. 12, pp. 567-569, 1976. 
[45] I. White, "Radiation from bends in optical waveguides: the volume-current method," IEE Journal on Microwaves, Optics and Acoustics, vol. 3, pp. 186-188, 1979.

[46] E.-G. Neumann, "Curved dielectric optical waveguides with reduced transition losses," in IEE Proceedings H (Microwaves, Optics and Antennas), 1982, pp. $278-280$.

[47] W. Gambling, H. Matsumura, C. Ragdale, and R. Sammut, "Measurement of radiation loss in curved single-mode fibres," Microwaves, Optics and Acoustics, IEE Journal on, vol. 2, pp. 134-140, 1978.

[48] E. Marcatili and S. Miller, "Improved relations describing directional control in electromagnetic wave guidance," Bell System Technical Journal, vol. 48, pp. 2161-2188, 1969.

[49] J.-M. Liu, Photonic devices: Cambridge University Press, 2005.

[50] P. Suchoski and R. V. Ramaswamy, "Design of single-mode step-tapered waveguide sections," Quantum Electronics, IEEE Journal of, vol. 23, pp. 205211, 1987.

[51] E. A. Marcatili, "Dielectric rectangular waveguide and directional coupler for integrated optics," Bell System Technical Journal, vol. 48, pp. 2071-2102, 1969.

[52] R. Baets and P. Lagasse, "Calculation of radiation loss in integrated-optic tapers and Y-junctions," Applied optics, vol. 21, pp. 1972-1978, 1982.

[53] A. Kumar, K. Thyagarajan, and A. K. Ghatak, "Analysis of rectangular-core dielectric waveguides: an accurate perturbation approach," Optics letters, vol. 8, pp. 63-65, 1983.

[54] A. Tavlove and S. Hagness, "Computational Electrodynamics: The FiniteDifference Time-Domain Method Artech House," Norwood, MA, 1995.

[55] K. S. Yee, "Numerical solution of initial boundary value problems involving Maxwell's equations in isotropic media," IEEE Trans. Antennas Propag, vol. 14, pp. 302-307, 1966.

[56] A. B. Fallahkhair, K. S. Li, and T. E. Murphy, "Vector finite difference modesolver for anisotropic dielectric waveguides," Journal of Lightwave Technology, vol. 26, pp. 1423-1431, 2008.

[57] K. Kruse, K. Walczak, N. Thomas, B. Swatowski, C. Demars, and C. Middlebrook, "Chemical inertness of UV-cured optical elastomers within the printed circuit board manufacturing process for embedded waveguide applications," in SPIE OPTO, 2014, pp. 898811-898811-11.

[58] R. Dangel, F. Horst, D. Jubin, N. Meier, J. Weiss, B. J. Offrein, et al., "Development of Versatile Polymer Waveguide Flex Technology for Use in Optical Interconnects," Journal of Lightwave Technology, vol. 31, pp. 3915-3926, 2013.

[59] R. Dangel, R. Beyeler, F. Horst, N. Meier, B. J. Offrein, B. Sicard, et al., "Waveguide technology development based on temperature-and humidityresistant low-loss silsesquioxane polymer for optical interconnects," in Optical Fiber Communication Conference, 2007, p. OThH2. 
[60] E. Zgraggen, I. M. Soganci, F. Horst, A. L. Porta, R. Dangel, B. J. Offrein, et al., "Laser Direct Writing of Single-Mode Polysiloxane Optical Waveguides and Devices," Journal of Lightwave Technology, vol. 32, pp. 3036-3042, 2014.

[61] R. A. Lawson, C.-T. Lee, W. Yueh, L. Tolbert, and C. L. Henderson, "Epoxide functionalized molecular resists for high resolution electron-beam lithography," Microelectronic Engineering, vol. 85, pp. 959-962, 2008.

[62] P. Tien and R. Ulrich, "Theory of prism-film coupler and thin-film light guides," JOSA, vol. 60, pp. 1325-1337, 1970.

[63] J. Harris, R. Shubert, and J. Polky, "Beam coupling to films," JOSA, vol. 60, pp. 1007-1016, 1970.

[64] J. N. Hilfiker, N. Singh, T. Tiwald, D. Convey, S. M. Smith, J. H. Baker, et al., "Survey of methods to characterize thin absorbing films with spectroscopic ellipsometry," Thin Solid Films, vol. 516, pp. 7979-7989, 2008.

[65] H. Baghsiahi, K. Wang, W. Kandulski, R. C. Pitwon, and D. R. Selviah, "Optical Waveguide End Facet Roughness and Optical Coupling Loss," Journal of Lightwave Technology, vol. 31, pp. 2959-2968, 2013.

[66] K. Kruse, N. Riegel, C. Demars, C. Middlebrook, and M. Roggemann, "Optical waveguide end roughness in correlation to optical coupling," in SPIE OPTO, 2012, pp. 82670N-82670N-7.

[67] A. Tynes, A. D. Pearson, and D. Bisbee, "Loss mechanisms and measurements in clad glass fibers and bulk glass," JOSA, vol. 61, pp. 143-153, 1971.

[68] B. Das, R. Ricken, and W. Sohler, "Integrated optical distributed feedback laser with Ti: Fe: Er: LiNbO 3 waveguide," Applied physics letters, vol. 82, pp. 15151517, 2003.

[69] L. Eldada, C. Xu, K. M. Stengel, L. W. Shacklette, and J. T. Yardley, "Laserfabricated low-loss single-mode raised-rib waveguiding devices in polymers," Lightwave Technology, Journal of, vol. 14, pp. 1704-1713, 1996.

[70] "Data format for positioning, line motion, and contouring control systems," in 6983-1: Numeric control of machines -- Program format and defiition of address words, ed: ISO, 1982.

[71] N. Riegel, C. Middlebrook, K. Kruse, and M. Roggemann, "Using the 3D beam propagation method to model the effects of lithographic roughness on the attenuation of highly multimodal polymer waveguides," Journal of Vacuum Science \& Technology B, vol. 31, p. 031206, 2013.

[72] L. Dellmann, C. Berger, R. Beyeler, R. Dangel, M. Gmur, R. Hamelin, et al., "120 Gb/s optical card-to-card interconnect link demonstrator with embedded waveguides," in Electronic Components and Technology Conference, 2007. ECTC'07. Proceedings. 57th, 2007, pp. 1288-1293.

[73] P. Calvert, "Inkjet printing for materials and devices," Chemistry of materials, vol. 13, pp. 3299-3305, 2001.

[74] R. Dangel, U. Bapst, C. Berger, R. Beyeler, L. Dellmann, F. Horst, et al., "Development of a low-cost low-loss polymer waveguide technology for parallel optical interconnect applications," in Biophotonics/Optical Interconnects and 
VLSI Photonics/WBM Microcavities, 2004 Digest of the LEOS Summer Topical Meetings, 2004, p. 2 pp.

[75] N. J. Riegel, "Optical Waveguides and Structures for Short Haul Optical Communication Channels within Printed Circuit Boards," 2013.

[76] X. Zhang, A. Hosseini, X. Lin, H. Subbaraman, and R. T. Chen, "Polymer-based hybrid-integrated photonic devices for silicon on-chip modulation and board-level optical interconnects," Selected Topics in Quantum Electronics, IEEE Journal of, vol. 19, pp. 3401115-3401115, 2013.

[77] X. Lin, T. Ling, H. Subbaraman, X. Zhang, K. Byun, L. J. Guo, et al., "Ultraviolet imprinting and aligned ink-jet printing for multilayer patterning of electro-optic polymer modulators," Optics letters, vol. 38, pp. 1597-1599, 2013.

[78] C.-Y. Lin, A. X. Wang, B. S. Lee, X. Zhang, and R. T. Chen, "High dynamic range electric field sensor for electromagnetic pulse detection," Optics express, vol. 19, pp. 17372-17377, 2011.

[79] X. Zhang, A. Hosseini, S. Chakravarty, J. Luo, A. K.-Y. Jen, and R. T. Chen, "Wide optical spectrum range, subvolt, compact modulator based on an electrooptic polymer refilled silicon slot photonic crystal waveguide," Optics letters, vol. 38, pp. 4931-4934, 2013.

[80] R. Baets and P. Lagasse, "Loss calculation and design of arbitrarily curved integrated-optic waveguides," JOSA, vol. 73, pp. 177-182, 1983.

[81] R. T. Farouki and J. Manjunathaiah, "G codes for the specification of Pythagorean-hodograph tool paths and associated feedrate functions on openarchitecture CNC machines," International Journal of Machine Tools \& Manufacture, vol. 39, p. 20, 1998.

[82] H. Nishihara, M. Haruna, and T. Suhara, Optical integrated circuits: McGrawHill New York, 1989.

[83] I. Papakonstantinou, K. Wang, D. R. Selviah, and F. A. Fernández, "Transition, radiation and propagation loss in polymer multimode waveguide bends," Optical Society of America, vol. 15, p. 11, 2007.

[84] K. L. Kruse and C. T. Middlebrook, "Fan-out routing and optical splitting techniques for compact optical interconnects using single-mode polymer waveguides," Journal of Modern Optics, pp. 1-10, 2014.

[85] D. Gloge, "Bending loss in multimode fibers with graded and ungraded core index," Applied Optics, vol. 11, pp. 2506-2513, 1972.

[86] D. Marcuse, "Gaussian approximation of the fundamental modes of graded-index fibers," JOSA, vol. 68, pp. 103-109, 1978.

[87] K. Kruse and C. Middlebrook, "Laser-direct writing of single mode and multimode polymer step index waveguide structures for optical backplanes and interconnection assemblies," Photonics and Nanostructures-Fundamentals and Applications, 2014.

[88] A. Syahriar, "A simple analytical solution for loss in S-bend optical waveguide," in RF and Microwave Conference, 2008. RFM 2008. IEEE International, 2008, pp. 357-360. 
[89] V. Subramaniam, G. N. De Brabander, D. H. Naghski, and J. T. Boyd, "Measurement of mode field profiles and bending and transition losses in curved optical channel waveguides," Lightwave Technology, Journal of, vol. 15, pp. 990997, 1997.

[90] B. Howley, X. Wang, R. T. Chen, and Y. Chen, "Experimental evaluation of curved polymer waveguides with air trenches and offsets," Journal of applied physics, vol. 100, p. 023114, 2006.

[91] X. Zhang, B. Lee, C.-y. Lin, A. X. Wang, A. Hosseini, and R. T. Chen, "Highly linear broadband optical modulator based on electro-optic polymer," Photonics Journal, IEEE, vol. 4, pp. 2214-2228, 2012.

[92] R. Soref, "The past, present, and future of silicon photonics," Selected Topics in Quantum Electronics, IEEE Journal of, vol. 12, pp. 1678-1687, 2006.

[93] T. Barwicz, H. Byun, F. Gan, C. Holzwarth, M. Popovic, P. Rakich, et al., "Silicon photonics for compact, energy-efficient interconnects [Invited]," Journal of Optical Networking, vol. 6, pp. 63-73, 2007.

[94] J. Galán, P. Sanchis, and J. Martí, "Low-loss coupling technique between SOI waveguides and standard single-mode fibers."

[95] L. Vivien and L. Pavesi, Handbook of silicon photonics: CRC Press, 2013.

[96] O. Mitomi, K. Kasaya, and H. Miyazawa, "Design of a single-mode tapered waveguide for low-loss chip-to-fiber coupling," Quantum Electronics, IEEE Journal of, vol. 30, pp. 1787-1793, 1994.

[97] S. M. Garner, S.-S. Lee, V. Chuyanov, A. Chen, A. Yacoubian, W. H. Steier, et al., "Three-dimensional integrated optics using polymers," Quantum Electronics, IEEE Journal of, vol. 35, pp. 1146-1155, 1999.

[98] A. Schleunitz and H. Schift, "Fabrication of 3D nanoimprint stamps with continuous reliefs using dose-modulated electron beam lithography and thermal reflow," Journal of Micromechanics and Microengineering, vol. 20, p. 095002, 2010 .

[99] I. Kaminow, W. Mammel, and H. Weber, "Metal-clad optical waveguides: analytical and experimental study," Applied Optics, vol. 13, pp. 396-405, 1974.

[100] S. Rashleigh, "Positive-permittivity-metal cladding: its effect on the modes of dielectric optical waveguides," Applied optics, vol. 15, pp. 2804-2811, 1976.

[101] W. Groh and A. Zimmermann, "What is the lowest refractive index of an organic polymer?," Macromolecules, vol. 24, pp. 6660-6663, 1991.

[102] A. Cho, A. Yariv, and P. Yeh, "Observation of confined propagation in Bragg waveguides," Applied Physics Letters, vol. 30, pp. 471-472, 1977.

[103] R. DeCorby, N. Ponnampalam, E. Epp, T. Allen, and J. McMullin, "Chip-scale spectrometry based on tapered hollow Bragg waveguides," Optics express, vol. 17, pp. 16632-16645, 2009.

[104] L. B. Soldano and E. C. Pennings, "Optical multi-mode interference devices based on self-imaging: principles and applications," Lightwave Technology, Journal of, vol. 13, pp. 615-627, 1995. 
[105] P. Yao, G. J. Schneider, B. Miao, J. Murakowski, D. W. Prather, E. D. Wetzel, et al., "Multilayer three-dimensional photolithography with traditional planar method," Applied Physics Letters, vol. 85, pp. 3920-3922, 2004.

[106] M. Totzeck, W. Ulrich, A. Göhnermeier, and W. Kaiser, "Semiconductor fabrication: Pushing deep ultraviolet lithography to its limits," nature photonics, vol. 1, pp. 629-631, 2007.

[107] L. Sun and G. L. Yip, "Analysis of metal-clad optical waveguide polarizers by the vector beam propagation method," Applied Optics, vol. 33, pp. 1047-1050, 1994.

[108] K. Rollke and W. Sohler, "Metal-clad waveguide as cutoff polarizer for integrated optics," Quantum Electronics, IEEE Journal of, vol. 13, pp. 141-145, 1977.

[109] X. Xu, S. Chen, J. Yu, and X. Tu, "An investigation of the mode characteristics of SOI submicron rib waveguides using the film mode matching method," Journal of Optics A: Pure and Applied Optics, vol. 11, p. 015508, 2009.

[110] G. Bhatt, P. Vivek, and B. Das, "Attenuation of TM-polarized light in metal coated SOI rib waveguides due to plasmonic absorption," 2010.

[111] F. Grillot, L. Vivien, S. Laval, and E. Cassan, "Propagation loss in single-mode ultrasmall square silicon-on-insulator optical waveguides," Journal of lightwave technology, vol. 24, p. 891, 2006.

[112] B. D. Gates and G. M. Whitesides, "Replication of vertical features smaller than 2 nm by soft lithography," Journal of the American Chemical Society, vol. 125, pp. 14986-14987, 2003.

[113] F. Hua, Y. Sun, A. Gaur, M. A. Meitl, L. Bilhaut, L. Rotkina, et al., "Polymer imprint lithography with molecular-scale resolution," Nano letters, vol. 4, pp. 2467-2471, 2004.

[114] M. Colburn, S. C. Johnson, M. D. Stewart, S. Damle, T. C. Bailey, B. Choi, et al., "Step and flash imprint lithography: a new approach to high-resolution patterning," in Microlithography'99, 1999, pp. 379-389.

[115] H. Lorenz, M. Despont, N. Fahrni, N. LaBianca, P. Renaud, and P. Vettiger, "SU8: a low-cost negative resist for MEMS," Journal of Micromechanics and Microengineering, vol. 7, p. 121, 1997.

[116] G. Li, J. Yao, H. Thacker, A. Mekis, X. Zheng, I. Shubin, et al., "Ultralow-loss, high-density SOI optical waveguide routing for macrochip interconnects," Optics Express, vol. 20, pp. 12035-12039, 2012.

[117] N. Shimoji, J. Yamakawa, and M. Shiino, "Development of Mini-MPO Connector," FURUKAWA ELECTRIC REVIEW, vol. 102, pp. 60-64, 1998.

[118] I. O'Connor, "Optical solutions for system-level interconnect," in Proceedings of the 2004 international workshop on System level interconnect prediction, 2004, pp. 79-88. 